\title{
The Effects of Freestream Turbulence on Serpentine Diffuser Distortion Patterns
}

Jesse Scott Johnson

Brigham Young University - Provo

Follow this and additional works at: https://scholarsarchive.byu.edu/etd

Part of the Mechanical Engineering Commons

\section{BYU ScholarsArchive Citation}

Johnson, Jesse Scott, "The Effects of Freestream Turbulence on Serpentine Diffuser Distortion Patterns" (2012). Theses and Dissertations. 3500.

https://scholarsarchive.byu.edu/etd/3500

This Thesis is brought to you for free and open access by BYU ScholarsArchive. It has been accepted for inclusion in Theses and Dissertations by an authorized administrator of BYU ScholarsArchive. For more information, please contact scholarsarchive@byu.edu, ellen_amatangelo@byu.edu. 
The Effects of Freestream Turbulence on Serpentine Diffuser Distortion Patterns

Jesse S. Johnson

A thesis submitted to the faculty of

Brigham Young University

in partial fulfillment of the requirements for the degree of

Master of Science

Steven E. Gorrell, Chair

R. Daniel Maynes

Scott L. Thomson

Department of Mechanical Engineering

Brigham Young University

December 2012

Copyright (C 2012 Jesse S. Johnson

All Rights Reserved 


\begin{abstract}
The Effects of Freestream Turbulence on Serpentine Diffuser Distortion Patterns

Jesse S. Johnson

Department of Mechanical Engineering

Master of Science
\end{abstract}

Serpentine diffusers have become a common feature in modern aircraft as they allow for certain benefits that are impossible with a traditional linear configuration. With the benefits, however, come certain disadvantages, namely flow distortions that reduce engine efficiency and decrease engine surge stability margins. These distortions are now being researched comprehensively to determine solutions for mitigating the adverse effects associated with them. This study investigates how varying the freestream turbulence intensity of the flow entering a serpentine diffuser affects the distortion patterns that are produced by the diffuser. Experiments were performed with a model serpentine diffuser on the Annular Cascade Facility of the Air Force Research Laboratory at Wright-Patterson Air Force Base. Hot wire anemometry was used to measure inlet turbulence, while static pressure probes located axially along the upper and lower surface of the model diffuser and total pressure probes located across the aerodynamic interface plane (AIP) were used to measure the distortion patterns of the flow passing through the diffuser.

Varying levels of inlet freestream turbulence, ranging from 0 to $4 \%$, were generated using square and round bar turbulence screens in three distinct test configurations. Axial static pressure measurements indicate that increasing turbulence slightly affects flow separation development downstream of the second turn. This effect is also seen at the AIP where the total pressure recovery increases with increasing level of inlet turbulence in the region of flow separation at the upper surface. The total pressure recovery along the lower surface is also seen to be increased with higher inlet turbulence. However, total pressure recovery increase across the entire AIP is almost negligible. Overall, the inlet freestream turbulence has a minor effect on the distortion patterns caused by the serpentine diffuser when compared with proven active inlet flow control methods.

Keywords: serpentine diffuser, turbomachinery, freestream turbulence, flow distortion, experimental fluid dynamics 


\section{ACKNOWLEDGEMENTS}

The author would like to thank his thesis advisor, Dr. Steven Gorrell, for making this opportunity possible and providing support throughout this effort. The author would also like to thank committee members, Dr. Scott Thomson and Dr. Daniel Maynes for their support. The author would like to thank and acknowledge the support, supervision, and technical input of Dr. William Copenhaver of the AFRL who assisted in this effort from start to finish. The author acknowledges the assistance and support of the entire CARL group at the AFRL who helped to make this work happen. The author would like to thank the United States Air Force for sponsoring this research. Finally, a great debt of gratitude is owed to the author's wife, Karen, for the great sacrifices she has made in support of this research. 


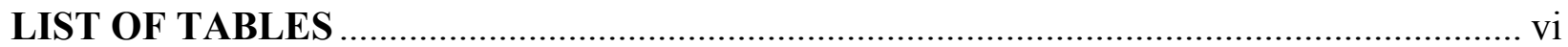

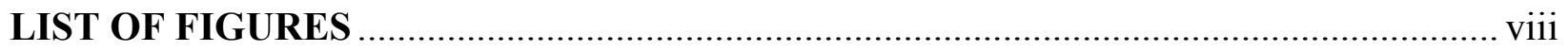

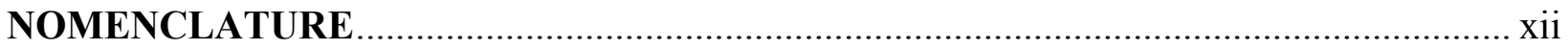

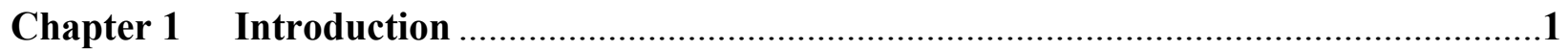

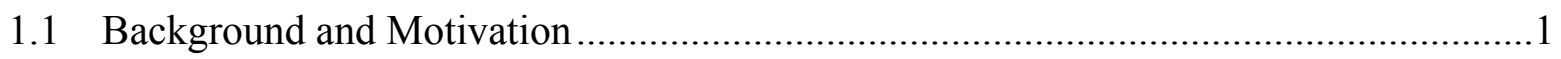

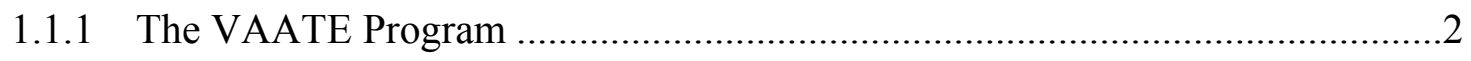

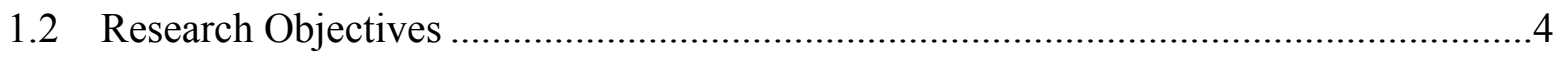

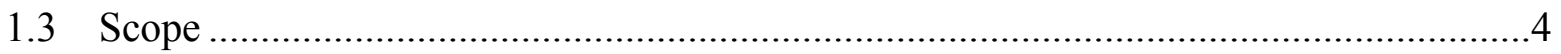

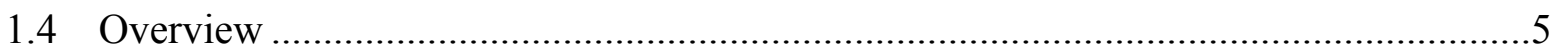

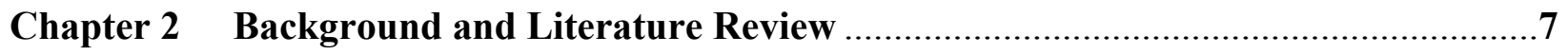

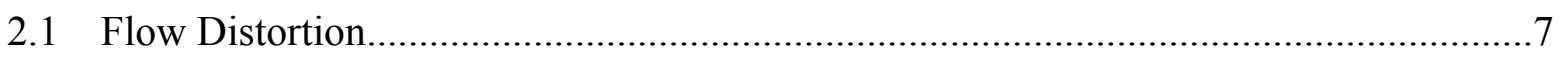

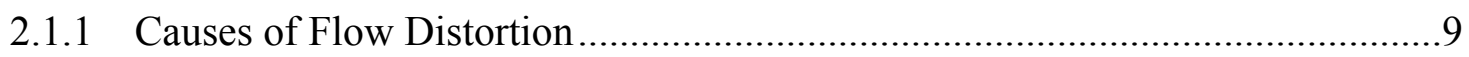

2.1.2 Effects of Flow Distortion........................................................................

2.2 Effects of Turbulence on Straight-Wall Diffuser Performance......................................13

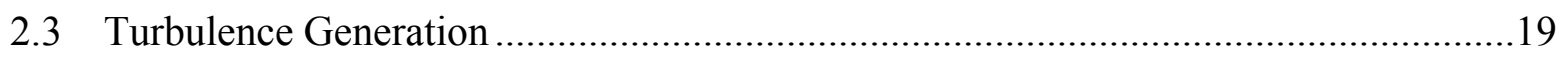

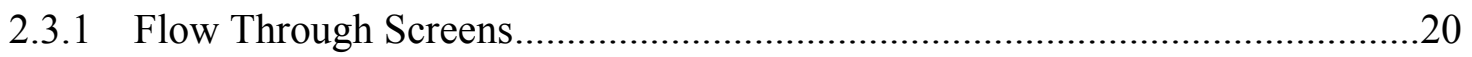

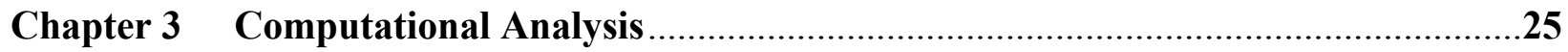

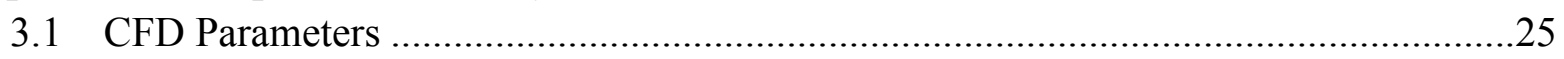

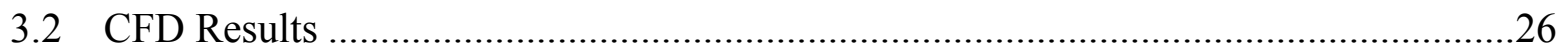

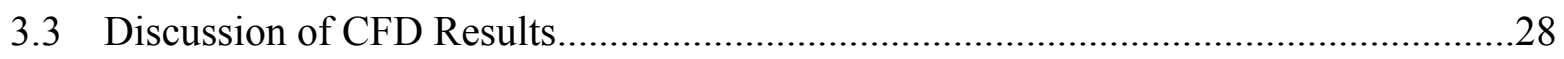

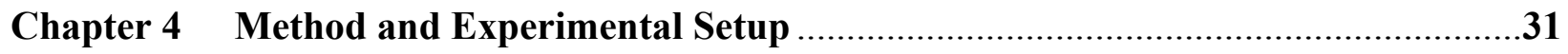

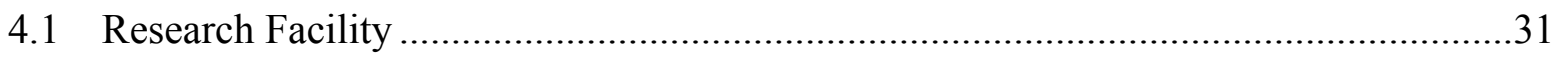

4.1.1 Annular Cascade Facility ........................................................................32

4.1.2 Serpentine Diffuser Test Model and Axial Static Pressure Measurement .........33

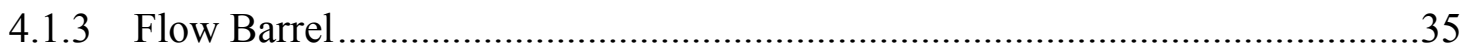

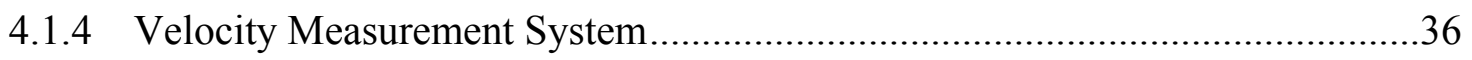

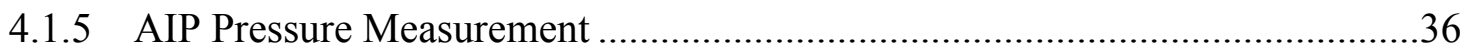

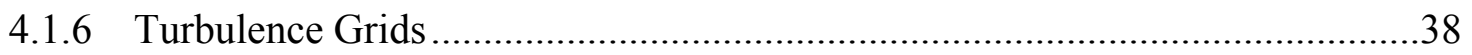

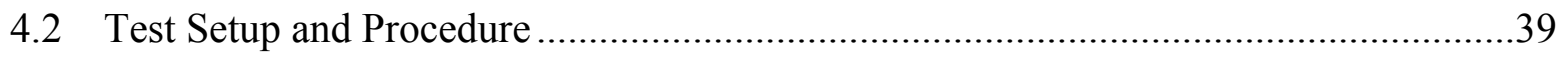

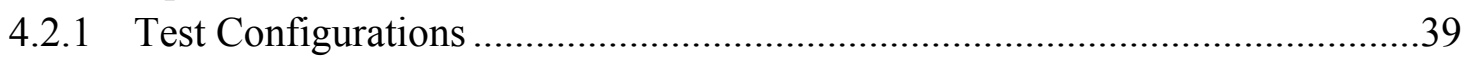

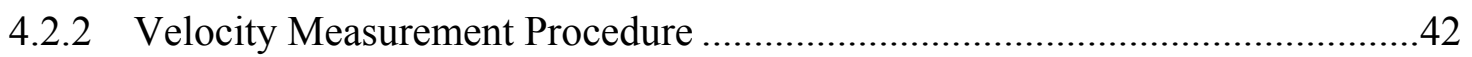

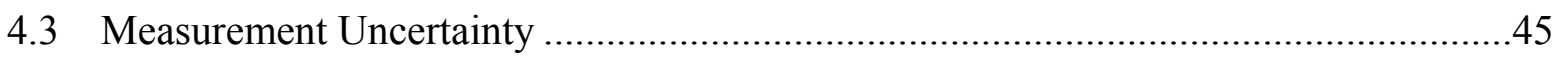




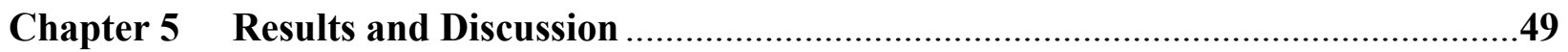

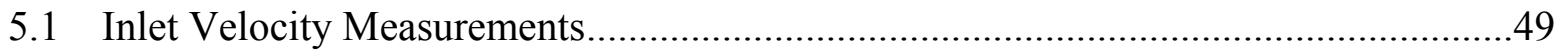

5.1.1 Mean Velocity Profiles........................................................................49

5.1.2 Fluctuating Velocity and Turbulence Intensity Profiles..................................53

5.1.3 Turbulence Generation without Flow Acceleration .......................................61

5.1.4 Off-Design Throat Mach Number Study.......................................................66

5.2 Axial Static Pressure Results....................................................................................6

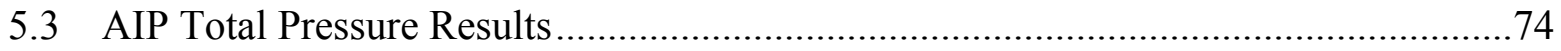

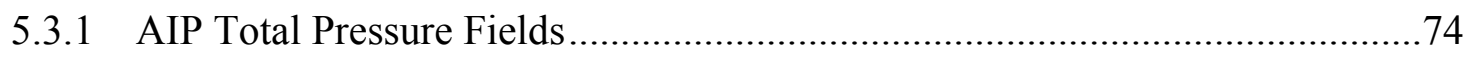

5.3.2 AIP Total Pressure Recovery .................................................................... 81

5.3.3 Off-Design Mach Number Total Pressure Recovery ......................................82

5.4 Discussion of Significance of Total Pressure Recovery Results.................................83

5.5 Comparison of CFD and Experimental Results .................................................85

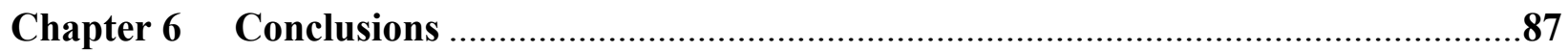

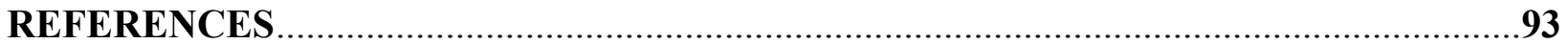

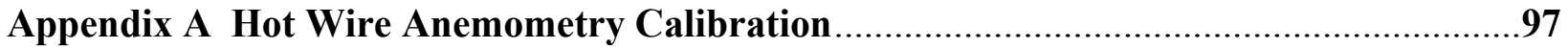

Appendix B Inlet Turbulence Scales and Statistics ...................................................103

Appendix C Axial Static Pressure Data at Off-Design Throat Mach Numbers ................109

Appendix D Re-Scaled Supplemental AIP Total Pressure Data Plots............................119

Appendix E Axial Wall Static Pressure Probe Locations ...............................................125 


\section{LIST OF TABLES}

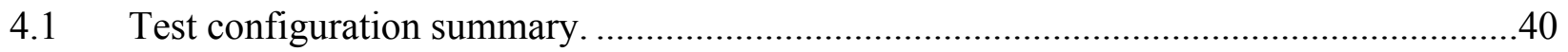

A.1 CTA settings for the two hot film probes used in experiments. ....................................98

B.1 Definitions of statistical moments for discrete sets of data. .........................................105

B.2 Calculated turbulence scales of velocity data at select locations for each turbulence

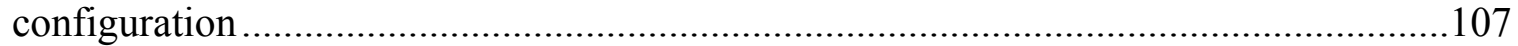

B.3 Calculated statistical moments of velocity data at select locations .............................108

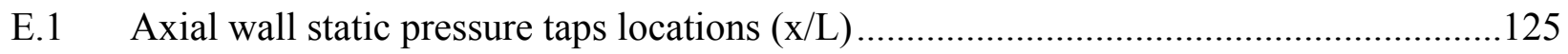




\section{LIST OF FIGURES}

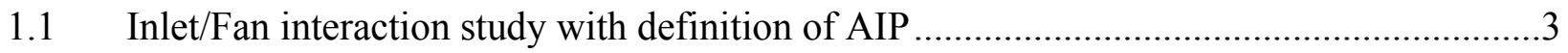

2.1 $1 /$ rev sinusoidal distribution of total pressure distortion at AIP used in CFD simulation to study effects of distortion on fan/compressor performance $\left[\frac{P t-\bar{P} t}{\bar{P} t}\right] \%$ [6].

2.2 Inlet separation caused by take-off which leads to distortion [7] .....................................

2.3 Serpentine inlets produce vortices at each of their two turns [7].....................................10

2.4 AIP total pressure distortion with (a) and (b) calculated by two distinct CFD models

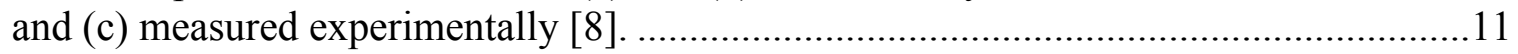

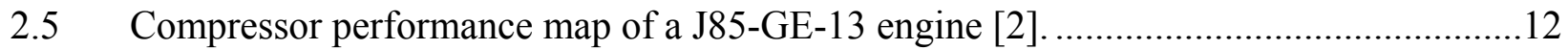

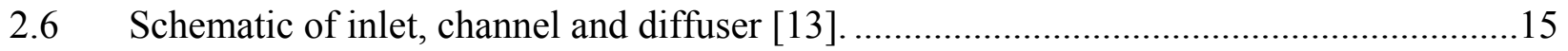

2.7 Schematic of rods used to generate turbulence [13] ........................................................16

2.8 Pressure recovery coefficient as a function of y-direction turbulence intensity [13] .......17

2.9 Pressure recovery coefficient as a function of streamwise turbulence intensity [13] .......17

2.10 Turbulence behavior downstream of turbulence grid at various cross-sectional positions compared with the correlation by Roach [25] ................................................22

3.1 Turbulence intensity field for two inlet turbulence intensities with turbulence level

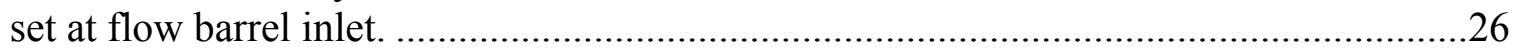

3.2 The turbulence intensity boundary conditions for two inlet turbulence intensities at

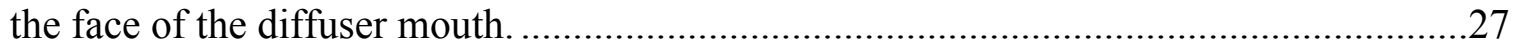

3.3 Total pressure and swirl distortion patterns at the AIP for each turbulence level with the turbulence specified at the face of the diffuser mouth.................................................29

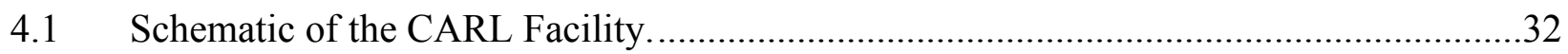

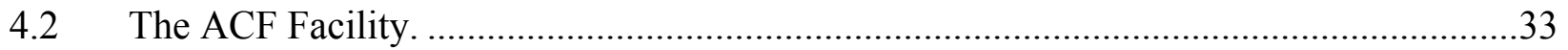

4.3 Normalized distance of certain locations along the axis of the serpentine diffuser (Note: profile of image not proportional to diffuser used in this experiment)....................34

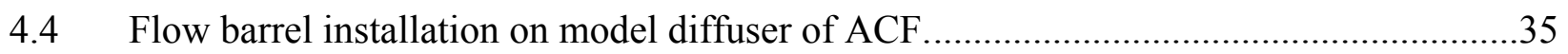

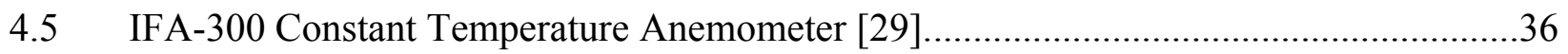

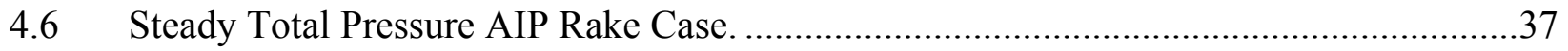

4.7 Contour of total pressure probe locations at AIP with sample total pressure field

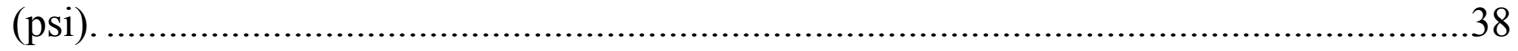

4.8 Exploded views of flow barrel assembly for each test configuration................................41

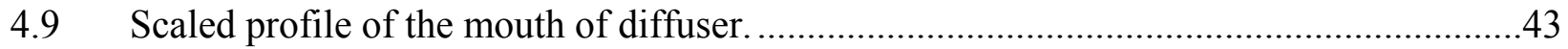

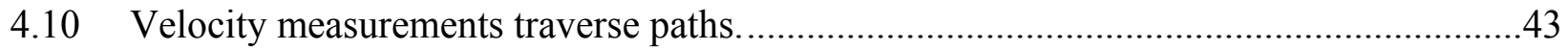

4.11 Flow barrel schematics (assembled in single screen configuration).................................45

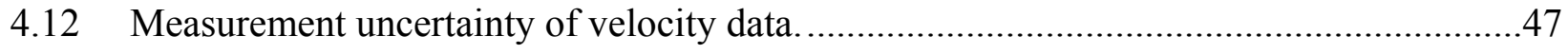


5.1 Mean velocity profiles at $\theta=0^{\circ}($ along $\mathrm{z} / \mathrm{W}=0), \mathrm{d}=0.75$ " for each test configuration.

5.2 Mean velocity profiles at $\theta=90^{\circ}$ (along $\mathrm{y} / \mathrm{H}=0.42$ ), $\mathrm{d}=0.75$ " for each test configuration.

5.3 urms profiles at $\theta=0^{\circ}$ (along $\mathrm{z} / \mathrm{W}=0$ ), $\mathrm{d}=0.75$ " for each test configuration..................54

5.4 urms profiles at $\theta=90^{\circ}$ (along $\mathrm{y} / \mathrm{H}=0.42$ ), $\mathrm{d}=0.75^{\prime}$ " for each test configuration............56

5.5 CFD velocity lines of flow entering model diffuser from flow barrel..............................57

5.6 Tu profiles at $\theta=0^{\circ}$ (along $\mathrm{z} / \mathrm{W}=0$ ), $\mathrm{d}=0.75$ " for each test configuration. …................58

5.7 Tu profiles at $\theta=90^{\circ}$ (along $\mathrm{y} / \mathrm{H}=0.42$ ), $\mathrm{d}=0.75^{\prime}$ " for each test configuration. ..............59

5.8 Mean velocity profiles at $\theta=0^{\circ}$ (along $\mathrm{z} / \mathrm{W}=0$ ), for the high screen configuration........62

5.9 Mean velocity profiles at $\theta=90^{\circ}$ for the high screen configuration. ..............................63

5.10 $T u$ profiles at $\theta=0^{\circ}$ (along $\mathrm{z} / \mathrm{W}=0$ ), for the high screen configuration..........................64

$5.11 T u$ profiles at $\theta=90^{\circ}$ for the high screen configuration...............................................65

5.12 Mean velocity at $\theta=90^{\circ}, \mathrm{z} / \mathrm{W}=0.41, \mathrm{y} / \mathrm{H}=0.42$ with varying throat Mach number......67

$5.13 T u$ at $\theta=90^{\circ}, \mathrm{z} / \mathrm{W}=0.41, \mathrm{y} / \mathrm{H}=0.42, \mathrm{~d}=0.75^{\prime}$ " with varying throat Mach number. ......68

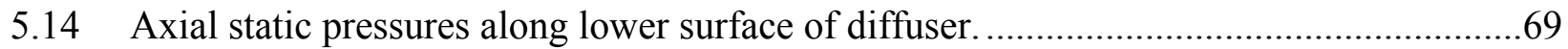

5.15 Rescaled axial static pressures along lower surface of diffuser........................................

5.16 Axial static pressures along upper surface of diffuser.....................................................

5.17 Re-scaled plot of the axial static pressures along upper surface of diffuser......................72

5.18 Further expanded view of axial static pressures along upper surface of diffuser...............73

5.19 Difference in axial static pressure between turbulence cases and baseline case ................73

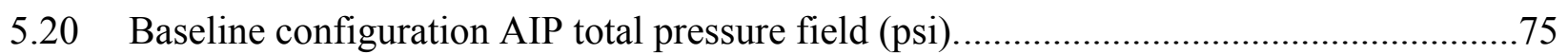

5.21 Single screen Configuration AIP total pressure field (psi) ...........................................76

5.22 Dual screens configuration AIP total pressure field (psi) ...............................................

5.23 High screen configuration AIP total pressure field (psi)...............................................

5.24 Total pressure difference between single screen and baseline cases (psi).......................78

5.25 Total pressure difference between dual screens and baseline cases (psi) .........................79

5.26 Total pressure difference between high screen and baseline cases (psi).........................79

5.27 Total pressure recovery percentage increase from baseline for each test

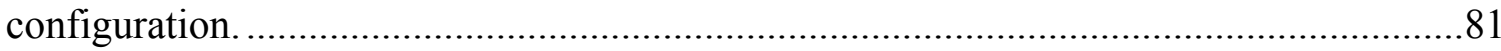

5.28 Total pressure recovery at off-design diffuser throat Mach numbers...............................83

A.1 Calibration data and 5th order polynomial curve-fit for sensor 005005 ...........................99

A.2 Calibration data and 5th order polynomial curve-fit for sensor 005006..........................100

A.3 Calibration data and 3rd order polynomial curve-fit for sensor 005005 ........................100

A.4 Calibration data and 3rd order polynomial curve-fit for sensor 005006 ........................101

C.1 Axial static pressures along upper surface of diffuser with varying Mach numbers for the baseline configuration.

C.2 Axial static pressures along upper surface of diffuser with varying Mach numbers for the single screen configuration. 


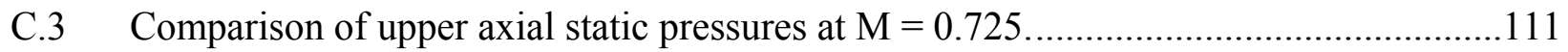

C.4 Scaled in comparison of upper axial static pressures at $\mathrm{M}=0.725 \ldots \ldots \ldots \ldots \ldots \ldots \ldots \ldots \ldots . . .111$

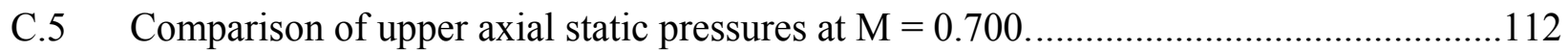

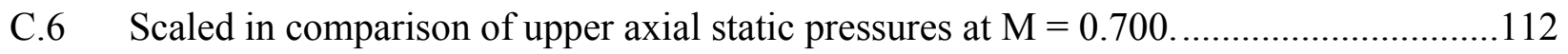

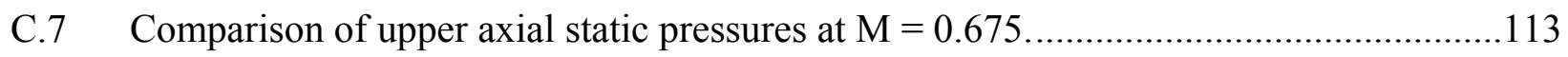

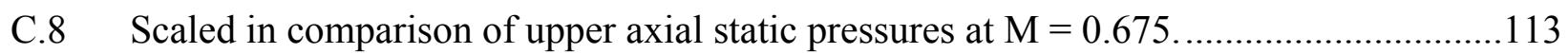

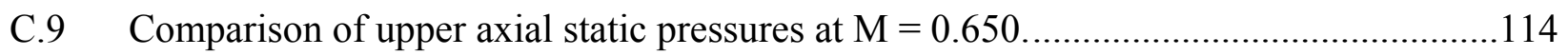

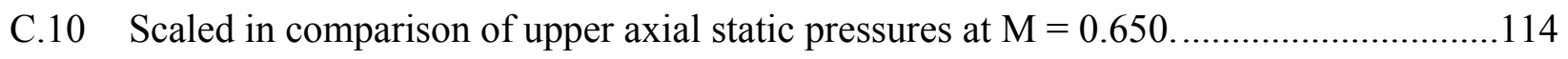

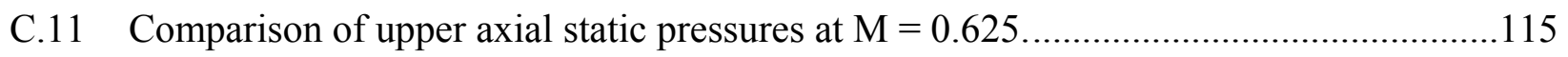

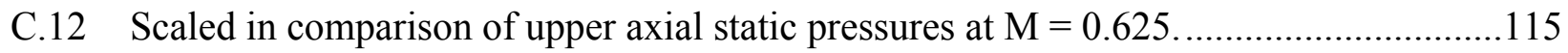

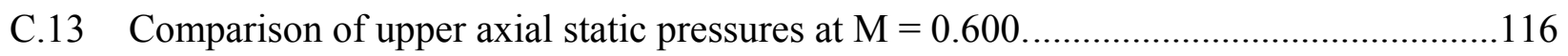

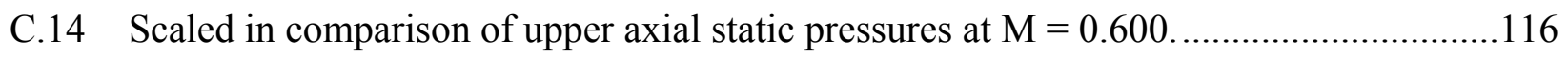

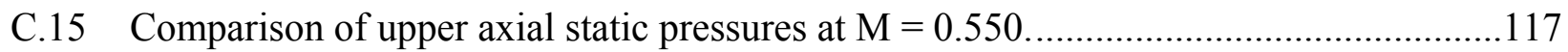

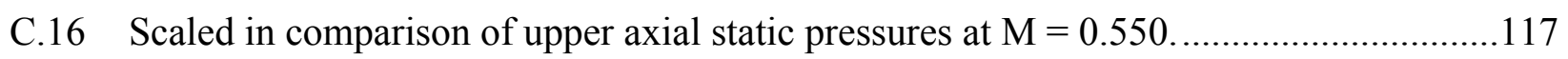

D.1 Total pressure difference between single screen and baseline (psi). ............................119

D.2 Positive total pressure difference between single screen and baseline (psi).................120

D.3 Negative total pressure difference between Single Screen and Baseline (psi)..............120

D.4 Total pressure difference between dual screens and baseline (psi). ............................121

D.5 Positive total pressure difference between dual screens and baseline (psi)...................121

D.6 Negative total pressure difference between dual screens and baseline (psi).................122

D.7 Total pressure difference between high screen and baseline (psi)...............................122

D.8 Positive total pressure difference between high screen and baseline (psi)...................123

D.9 Negative total pressure difference between high screen and baseline (psi)...................123 


\section{NOMENCLATURE}

\begin{tabular}{|c|c|}
\hline AFRL & Air Force Research Laboratory \\
\hline AIP & Aerodynamic Interface Plane \\
\hline UAS & Unmanned Aerial Systems \\
\hline VAATE & Versatile Affordable Advanced Turbine Engines \\
\hline HIT & High Impact Technology \\
\hline CFD & Computational Fluid Dynamics \\
\hline Pt & Total pressure \\
\hline $2 \theta$ & Total straight-wall diffuser divergence angle \\
\hline$\lambda_{\mathrm{x}}$ & Integral length scale \\
\hline$\delta^{*}$ & Boundary displacement thickness \\
\hline$k$ & Turbulent kinetic energy \\
\hline$T$ & Flux of energy \\
\hline$P$ & Production of turbulent kinetic energy \\
\hline$\varepsilon$ & Dissipation of turbulent kinetic energy \\
\hline$T u$ & Turbulence intensity \\
\hline$d$ & Bar diameter \\
\hline M & Mesh spacing \\
\hline$P$ & Static pressure loss/drop \\
\hline$q$ & Upstream dynamic pressure \\
\hline$\beta$ & Grid porosity \\
\hline P0 & Total pressure (Figure 3.3) \\
\hline CARL & Compressor Aero Research Laboratory \\
\hline $\mathrm{A} / \mathrm{D}$ & Analog-to-digital \\
\hline $\mathrm{ACF}$ & Annular Cascade Facility \\
\hline $\mathrm{x} / \mathrm{L}$ & Normalized axial/streamwise displacement measured from diffuser throat \\
\hline $\mathrm{y} / \mathrm{W}$ & Normalized y-direction displacement \\
\hline $\mathrm{z} / \mathrm{H}$ & Normalized z-direction displacement \\
\hline $\mathrm{d}$ & Axial/streamwise displacement measured from diffuser mouth face \\
\hline$\theta$ & Azimuthal probe insertion coordinate \\
\hline$u$ & Variable uncertainty \\
\hline $\mathrm{U}$ & Mean streamwise velocity \\
\hline$u_{r m s}$ & $\begin{array}{l}\text { Standard deviation of streamwise velocity or RMS of streamwise fluctuating } \\
\text { velocity }\end{array}$ \\
\hline RMS & Root-mean-square \\
\hline$u^{\prime}$ & Streamwise fluctuating velocity \\
\hline $\mathrm{P}$ & Static pressure \\
\hline$P_{0}$ & Inlet total pressure \\
\hline$v$ & Velocity \\
\hline
\end{tabular}




$\begin{array}{ll}\rho & \text { Density } \\ \mathrm{M} & \text { Mach number } \\ \text { AIFC } & \text { Active Inlet Flow Control } \\ \text { VG } & \text { Vortex Generator } \\ \text { EGO } & \text { Efficient global optimization } \\ \Lambda & \text { Integral length scale } \\ \tau_{\Lambda} & \text { Integral time scale } \\ \lambda & \text { Taylor microscale } \\ \tau_{\lambda} & \text { Taylor time scale } \\ \eta & \text { Kolmogorov length scale } \\ R(s) & \text { Autocorrelation function across time } \\ f(r) & \text { Autocorrelation function across space } \\ \sigma & \text { Standard deviation }\end{array}$




\section{CHAPTER 1. INTRODUCTION}

\subsection{Background and Motivation}

Using a serpentine shaped diffuser as an inlet on a gas turbine engine has become increasingly popular recently. Its rise in popularity can be attributed to some attractive benefits that include but are not limited to: virtually limitless engine configurability options, increased stealth due to zero line-of-sight to the combustor as well as a smaller radar cross-section, and an increase in thrust-to-weight ratio due to a reduction in overall aircraft length [1]. These benefits are especially attractive for Unmanned Aerial Systems (UAS) applications.

However, the intrinsic shape of the serpentine inlet, or diffuser, creates some negative effects that must be addressed. Distortion is produced as air passes through the bends of the inlet and enters the compressor. Flow distortion is defined as a non-uniformity, or unexpected profile disturbance, in the total pressure, total temperature, or velocity distribution over the inlet crosssection [2]. These distortions have adverse effects on the efficiency and functionality of the engine. Flow distortion entering the fan or compressor can cause compressor stall or surge.

Distortion can be classified into three types: pressure, temperature and swirl [3]. In a traditional linear engine inlet, it is typically assumed that the total temperature and total pressure have high recovery coefficients and maintain relative uniformity. With a serpentine inlet the recovery coefficients are significantly lower and the distortion patterns create non-uniform flow. In order to fully realize the benefits of serpentine inlets, the engine design must account for these distortions, either by implementing a type of flow control in the diffuser to reduce the scale of 
distortion or by incorporating new compressor design implementations based on known distortion patterns.

\subsubsection{The VAATE Program}

The Versatile Affordable Advanced Turbine Engines (VAATE) Program is a multidisciplinary effort that is collaborative across several organizations including the Air Force, Army, Navy, NASA, DOE and private industry. The goals of VAATE are to reduce the development, production and maintenance costs, increase fuel efficiency and increase the power to weight ratio of aircraft engines through stable, long-term foundational research and development.

The Fan and Compressor branch of the Air Force Research Laboratory (AFRL) working under the umbrella of VAATE has undertaken the task of studying the distortion effects created by a serpentine inlet. The program's name is High Impact Technology (HIT) Research Program. The main objectives of the HIT Research Program are to define the distortion created by a compact serpentine inlet, using both experimental and computational approaches, determine the effect of this distortion on compressor performance, and to develop flow control techniques that effectively mitigate the detrimental interactions effects on the fan. Two experimental facilities will be used to complete this investigation: the isolated diffuser test stand which will be used to measure and define the distortion patterns that are generated by the serpentine diffuser and the compressor facility which features a test compressor and will be used to investigate the effects of the distortion patterns generated by the serpentine diffuser on the performance of the compressor.

Using advanced facility capabilities, measurements are being taken in a specific serpentine-shaped diffuser experimental apparatus that has been designed through the VAATE 
collaboration for commission on modern aircraft. An objective of the experimental studies is to be able to fully quantify the pressure and swirl distortions created by the diffuser. Additionally, computational analyses are being performed in conjunction with the experimental studies; the experimental studies will provide for validation of the simulations.

Eventually, distortion screens will be placed in front of the compressor test facility in attempts to reproduce individual types of distortion and in the same manner and magnitude as the distortion that is measured at the exit of the compact serpentine diffuser. This will allow for investigation of the individual effect that each type of distortion has on the performance of the compressor. Types of flow distortion include total pressure distortion, total temperature distortion and swirl. In order to facilitate this interaction study, an Aerodynamic Interface Plane (AIP), located at the exit of the diffuser and entrance to the compressor, is used "to define distortion and performance at the aerodynamic interface between the inlet and the engine" [4] (see Figure 1.1).

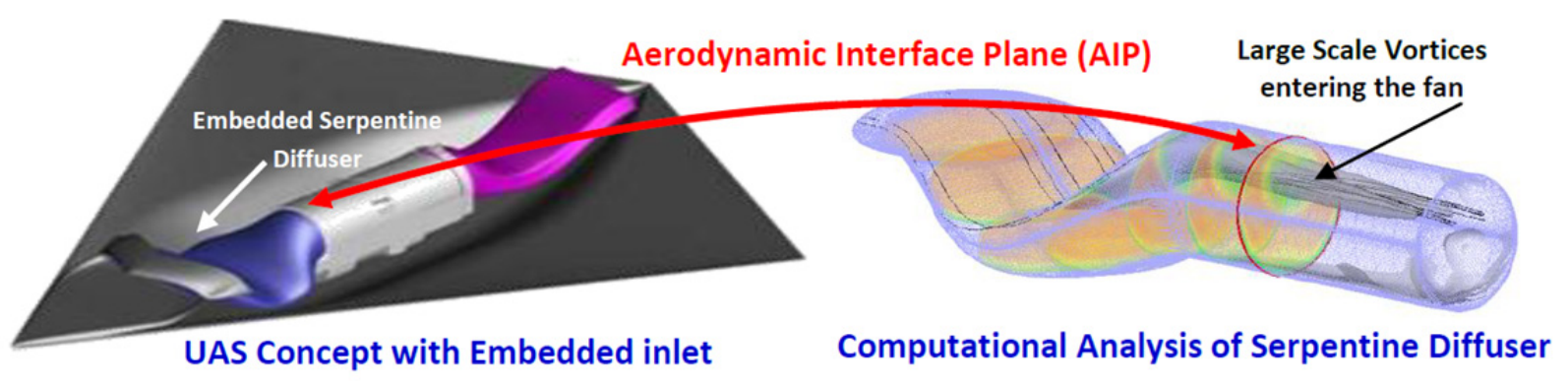

Figure 1.1: Inlet/Fan interaction study with definition of AIP. 


\subsection{Research Objectives}

The primary objective of this research is to determine the effects of freestream turbulence on the performance of a serpentine diffuser. The primary motivation behind this research is to determine whether the effects of freestream turbulence are sufficiently significant such that it should be accounted for during the HIT research program. This research will also contribute to the current research to analyze flow physics of air passing through serpentine diffusers. In order to perform this research, a suitable method of turbulence generation was needed along with a technique to measure the level of turbulence. Additionally, measurements were taken to quantify the level of performance of the serpentine diffuser. The performance of the serpentine diffuser in this study is defined by the flow physics of the air traveling through the diffuser and the distortion patterns present at the exit of the diffuser that effect the total pressure recovery of the diffuser. The performance of the diffuser is considered to be increasing with increasing flow uniformity, or decreasing flow distortion, at the AIP resulting in an increase in total pressure recovery.

\subsection{Scope}

Measurements were taken for three distinct configurations of distribution of turbulence intensity and compared with baseline measurements taken with only the background turbulence intensity inherent in the flow. Radial profiles of the mean velocity field of the flow entering the model diffuser were measured in order to quantify the level of turbulence intensity for each case. Statistical analysis of the turbulence field is included in Appendix B for each case. Velocity measurements were taken at varying locations to investigate the effect of flow acceleration on freestream turbulence decay. 
In order to quantify the diffuser performance, static pressure measurements were taken along the axis on the upper and lower surfaces of the diffuser. This measurement was used to discern whether or not turbulence has an effect on flow separation that occurs inside of the diffuser. Total pressure measurements were also taken at the AIP of the diffuser to quantify the total pressure distortion.

This study also briefly examined the effects of turbulence on serpentine diffuser performance at varying levels of diffuser throat Mach number. In this experiment, turbulence intensity at only a single location was used as opposed to full velocity profiles.

Velocity measurements at the AIP were not taken to quantify swirl distortion nor were temperature measurements taken at the AIP to quantify total temperature distortion at the three distinct turbulence configurations.

The experimental results of serpentine diffuser performance were compared to computational studies of similar parameters. Computational Fluid Dynamics (CFD) with STARCCM+ was used to provide the computational results for comparison with the experimental data. The CFD analysis was performed by a colleague at the AFRL, Dr. Darius Sanders, who is already heading up the computational side of the comprehensive diffuser studies.

\subsection{Overview}

This document is organized as follows: Chapter 2 gives background on serpentine inlets and distortion, previous efforts to examine effects of turbulence on diffuser performance, and turbulence generation. Chapter 3 presents results from a brief computational analysis of the effects of freestream turbulence on serpentine diffuser performance. Chapter 4 provides information on the hardware used to create the desired flow conditions and take measurements, a 
detailed description of the facility that was used and the test plan that was followed. Chapter 5 gives the results of the experiments along with some discussion of those results. Chapter 6 provides a summary and conclusions, and makes recommendations for future research. 


\section{CHAPTER 2. BACKGROUND AND LITERATURE REVIEW}

In this chapter, a review of important principles and studies that are related to this work and which provide background to the present research are presented. Flow distortion and its effect on jet engine performance is the underlying concern for this study and therefore a deep background investigation is provided. A discussion on a study that looked at the effects of freestream turbulence on straight-wall diffuser performance is included. Reviewing this study allows for a comparison between the effects of freestream turbulence on straight-wall diffuser and serpentine diffuser performance. Finally, a summary of the background research that took place to investigate methods to experimentally generate turbulence for this study is included.

\subsection{Flow Distortion}

Flow distortion and its effects on aircraft engine installations have been of concern since the dawn of jet engines. In theory, a typical jet engine with a circular straight inlet is assumed to have little distortion during steady flight. In practice however, this is rarely the case due to many factors that will be discussed in section 2.1.1. With the advent of serpentine and other nonstraight jet engine configurations, inlet flow distortion has become a primary concern for engine performance. Flow distortion can be divided into three groups: total pressure distortion, total temperature distortion and swirl distortion. Increases in inlet flow distortion have the effect of decreasing the efficiency and the stability margin of the engine, which is discussed in more detail in section 2.1.2. 
Total pressure distortion is defined by non-uniformities in the azimuthal coordinate of the planar total pressure profile within the engine. An example of planar total pressure distortion in front of a compressor is shown in Figure 2.1. Likewise, total temperature distortion is defined by non-uniformities in the azimuthal coordinate of the planar total temperature profile. Bulk swirl distortion is defined as the rotation of the complete flow field entering into the engine. There are also peak swirl and vortex swirl distortions which pertain to vortices created near, and sometimes due to, compressor rotors [5].

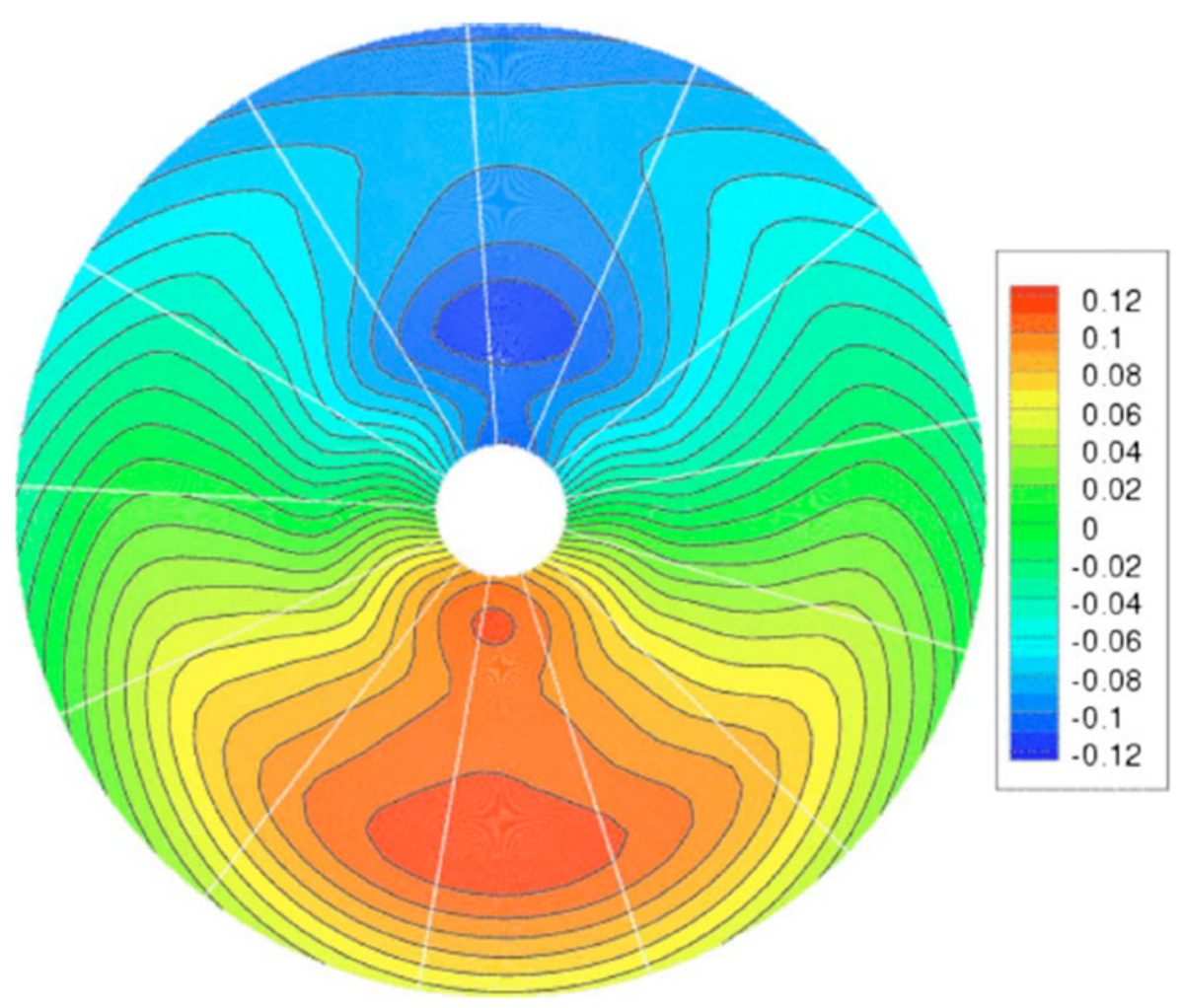

Figure 2.1: 1/rev sinusoidal distribution of total pressure distortion at AIP used in CFD simulation to study effects of distortion on fan/compressor performance $\left[\frac{P t-\bar{P} t}{\bar{P} t}\right] \%[6]$. 


\subsubsection{Causes of Flow Distortion in Jet Engines}

For a typical commercial aircraft engine located far out on the wing, flow distortion is generally assumed to be minimal during steady cruise conditions. For essentially any other case, flow distortion becomes a significant concern. Take-off, maneuvering, and landing are examples of scenarios where flow distortion is developed as air enters the engine. Figure 2.2 illustrates how distortion can develop from inlet separation that occurs during takeoff. Some aircraft have engines located near the fuselage which can cause uneven boundary layer growth entering the diffuser resulting in a total pressure distortion.

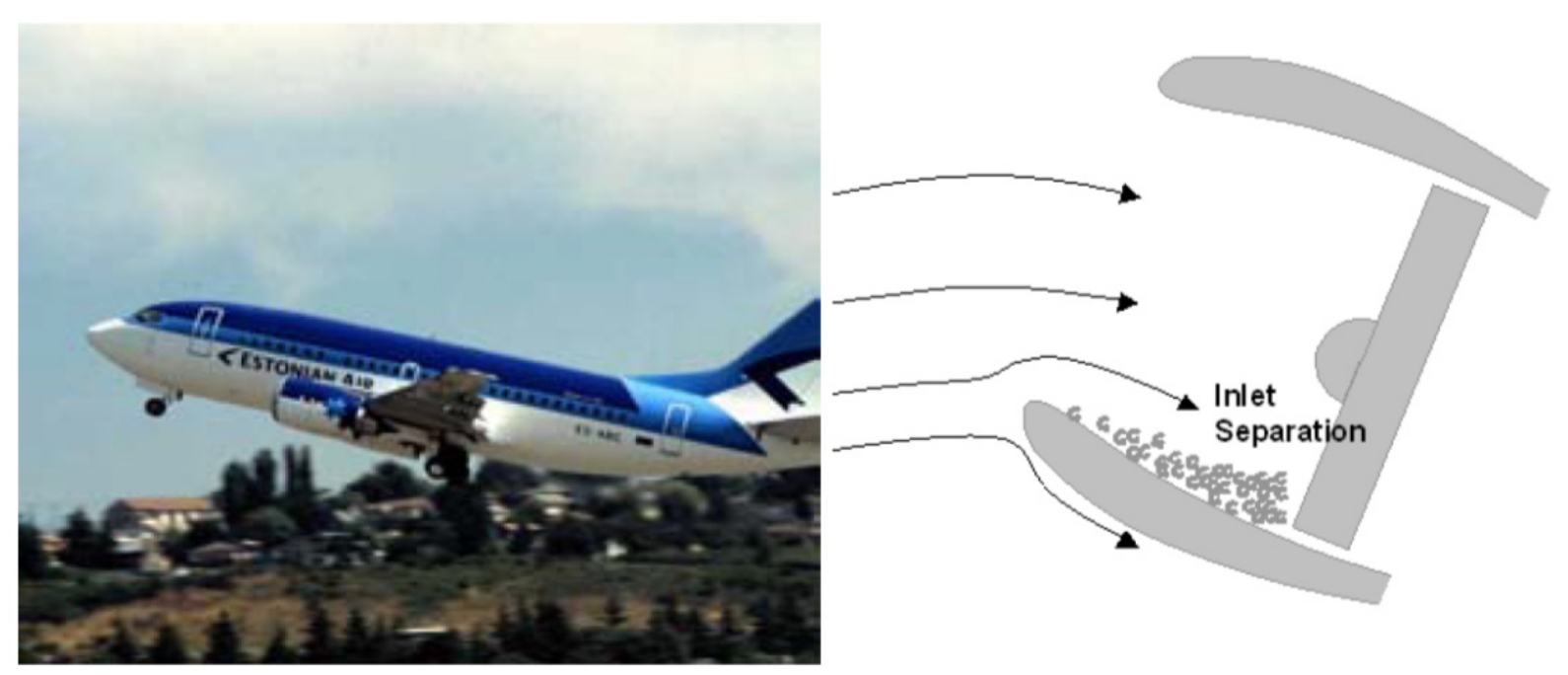

Figure 2.2: Inlet separation caused by take-off which leads to distortion [7].

Total temperature distortion can be caused by "weapon gas ingestion, vertical take-off and landing, steam ingestions during aircraft carrier operations, and mid-air refueling (gas leakage consumed by fan)" [3]. Total temperature distortion is also generated in the compressor as fan rotors responds to incoming total pressure distortion [6]. 
Although it is often the case where swirl distortion is created in conjunction with total pressure distortion, either by sharing a common cause or by total pressure distortion itself being the cause of swirl, it is also quite possible to have swirl distortion without significant total pressure distortion. Some of the typical causes of swirl distortion, in addition to some of the aforementioned causes, include wake ingestion from wings of other aircraft at busy airports and nacelle droop. It is also possible for a vortex to be formed between the ground and the rotating engine on the runway, which is then ingested into the engine causing multiple types of flow distortion [5].

Serpentine diffusers cause all three types of flow distortion even during steady cruise conditions with uniform inlet flow. The serpentine inlet comprises two turns that the flow must follow which can lead to separation and vorticity in each turn (see Figure 2.3). The flow separation that occurs around each bend leads to pockets of low mean velocity flow.
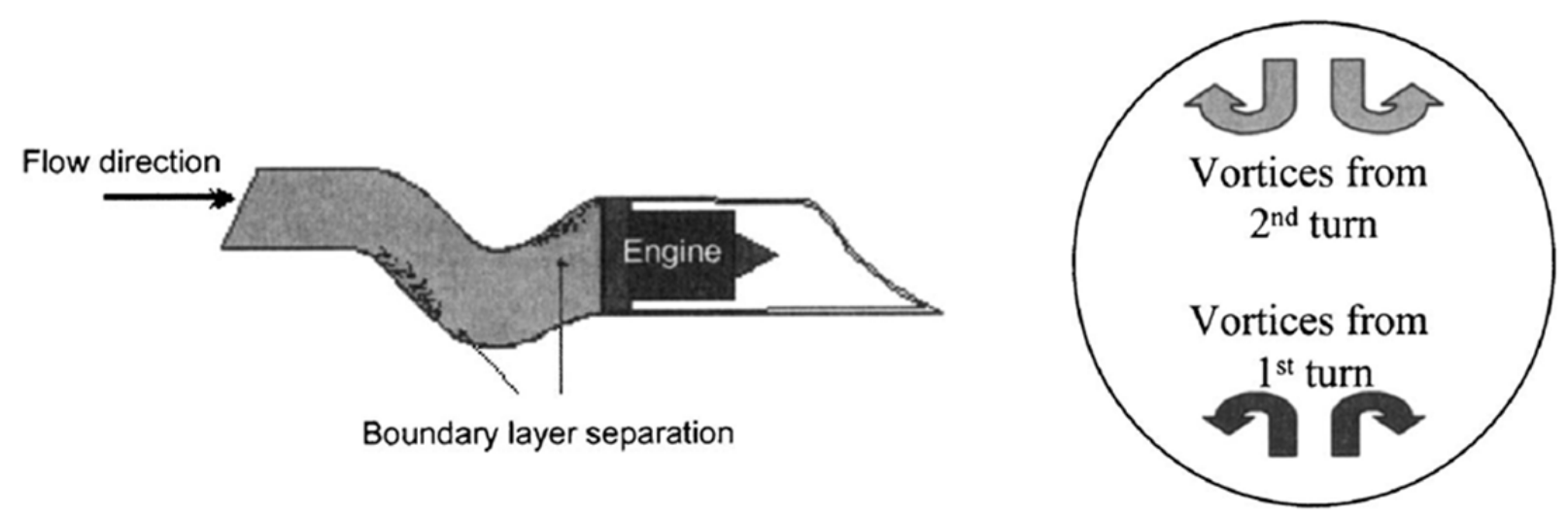

Figure 2.3: Serpentine inlets produce vortices at each of their two turns [7].

The separation that is produced at the final turn is especially concerning because it occurs immediately before the flow enters the compressor. Examples of the total pressure loss after a 
serpentine inlet at the engine face, or AIP, are presented in Figure 2.4. The effect of separated flow can be seen in each of these examples near the upper surface. This amount of total pressure loss will considerably increase the risk of compressor stall which is discussed in more detail in section 2.1.2.

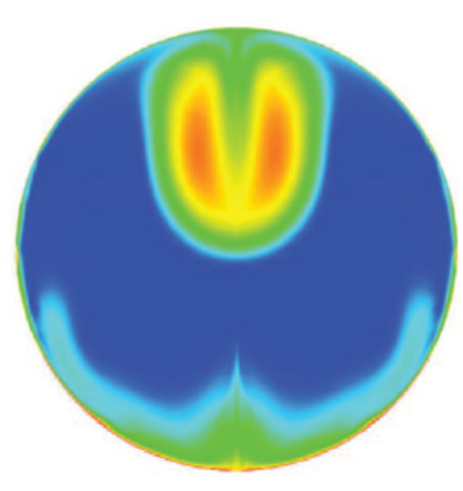

(a)

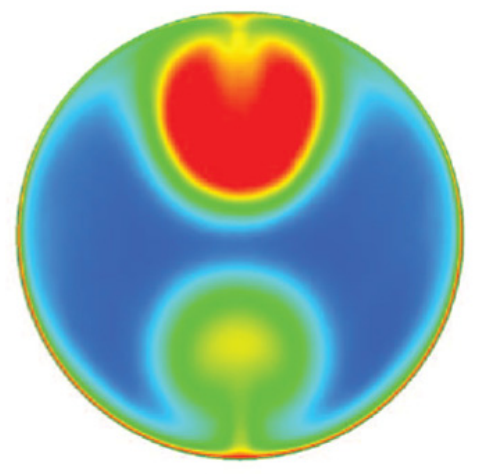

(b)

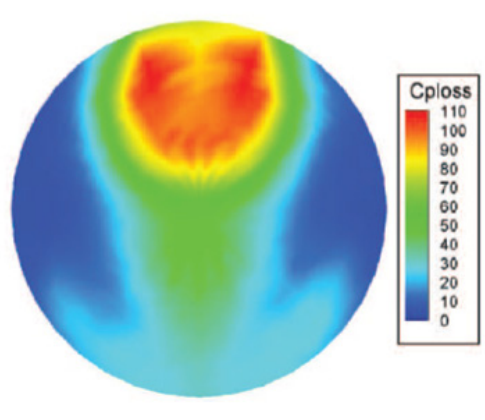

(c)

Figure 2.4: AIP total pressure distortion with (a) and (b) calculated by two distinct CFD models and (c) measured experimentally [8].

\subsubsection{Effects of Flow Distortion on Jet Engines}

While many aforementioned benefits are derived from the incorporation of serpentine inlets in aircraft systems, the flow distortion they cause entering the face of the engine produces negative effects. Compromising the stability, performance and even structural integrity of the engine, flow distortion is a concern that deserves careful investigation.

Flow separation occurs around the bends of a serpentine diffuser resulting in pockets of low mean velocity flow. Those pockets of low mean velocity flow can cause rotating stall, which is a local disruption of airflow in a compressor rotor that reduces compression effectiveness. Localized rotating stall can eventually trigger complete compressor surge. Compressor surge is a complete breakdown in compression which results in the downstream 
compressed air to reverse flow, causing a loss of engine thrust. Compressor surge can lead to a compromise of the structural integrity of the engine components [9], although an engine can recover from a surge event. At the very least, inlet distortion will reduce the stability margin of the operation of a jet engine. A typical compressor map schematic with the surge line and stability margin indicated for a J85-GE-13 engine is shown in Figure 2.5.

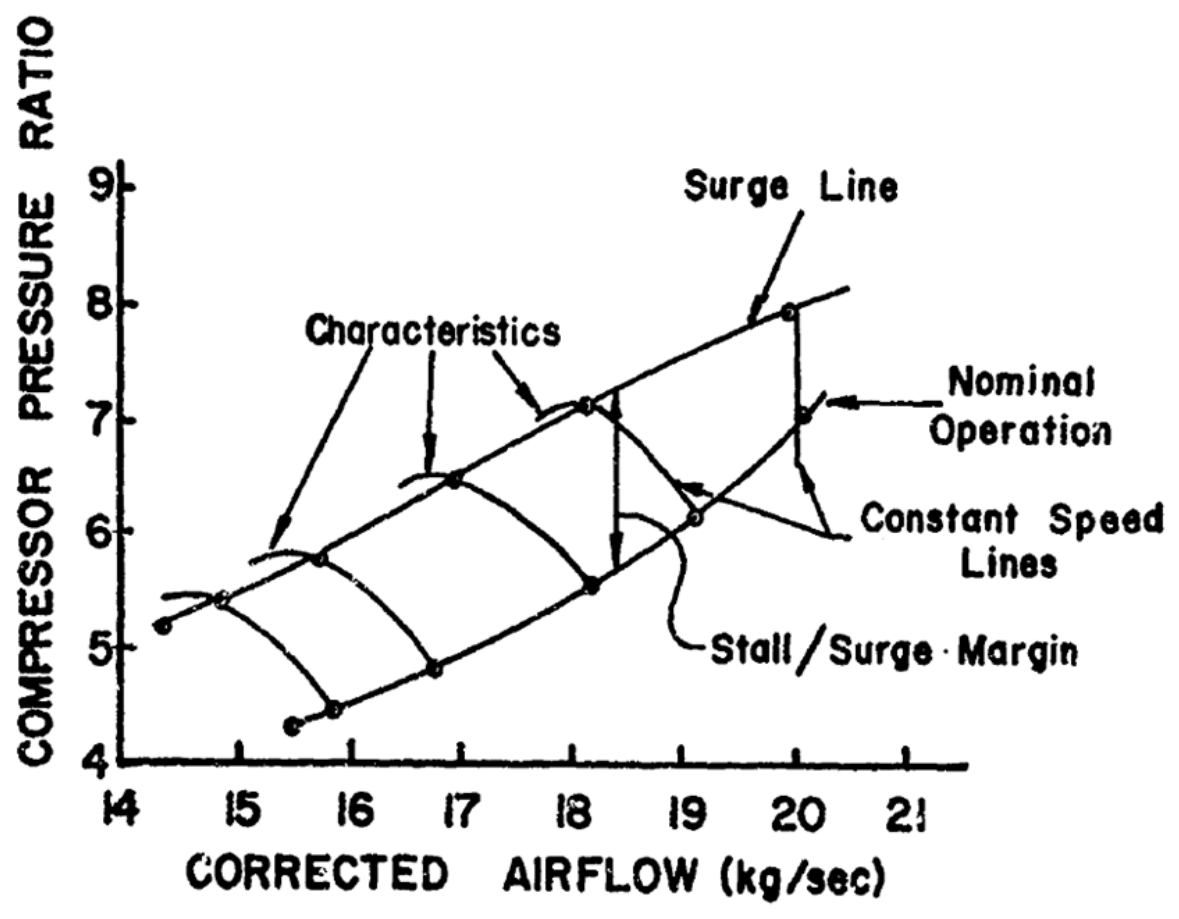

Figure 2.5: Compressor performance map of a J85-GE-13 engine [2].

A compressor performance map is used by engineers in the design process of a turbine engine. The nominal operation line is dependent on the magnitude of the cross-sectional area of the turbine. The compressor peak efficiency usually lies too close to the surge line at which to operate with a reasonable stability margin [2]. Flow distortion that enters the engine will move the nominal operation of the engine up the constant speed line towards the surge line. The stability margin (annotated stall/surge margin in Figure 2.5) is maintained for safety precautions 
to avoid compressor surge. Hence the compressor map is useful in designing a nominal operation with an appropriate stability margin to account for possible inlet distortion. In UAS applications, the stability margin can be reduced due to the lack of risk to human life in the event of failure. However, most UAS aircrafts employ distortion producing serpentine inlets thereby eliminating the potential efficiency advantage unless the effects of serpentine distortion can be reduced or mitigated.

\subsection{Effects of Turbulence on Straight-Wall Diffuser Performance}

While many studies have investigated the flow distortion produced by serpentine diffusers, research on the effects of freestream turbulence on the flow phenomena through diffusers is relatively scarce. Meanwhile, this work's investigation of the effects of freestream turbulence on serpentine diffuser performance is unprecedented. Many studies have been performed to investigate the effect on flow separation and pressure recovery when placing a rod or cylinder in front of the mouth of a diffuser such as those performed by Moore et al. [10], Waitman et al. [11], and Sajben et al. [12]. Each of them concludes that the added turbulence intensity reduces separation in the diffuser and increases the pressure recovery at the exit of the diffuser. Pressure recovery is defined as the ratio of outlet pressure to inlet pressure of a diffuser or inlet. An ideal diffuser or inlet has a pressure recovery of 1, but factors including wall friction and flow separation reduce the total pressure at the exit. Although placing a rod or cylinder in front of the diffuser will generate turbulent flow, the lack of downstream distance between the rod or cylinder and the diffuser means the turbulent flow has not had the opportunity to mix into the whole flow field. 
In a study performed by Hoffman [13], the effect of well-defined freestream turbulence on subsonic two-dimensional diffuser performance was investigated. "Diffuser performance" is again defined as the static pressure recovery factor of the diffuser. Hence, this study looks at the effect that varying levels of freestream turbulence have on the static pressure recovery factor, or pressure losses, of a straight-wall diffuser. The study assumed static pressure is uniform in plane throughout the diffuser and therefore did not observe distortions in the flow. Hoffman confirmed that increasing the freestream turbulence delayed separation in the diffuser and increased the static pressure recovery.

In the experiment, Hoffman used a flexible diffuser (see Figure 2.6) and various sets of round rods arranged circumferentially at an equal distance from the inlet to the experimental rig (see Figure 2.7). In three of the rod set configurations the rods were aligned horizontally as opposed to vertically like the rest of the 17 total rod sets. A channel exists between the rods and the diffuser to allow for the turbulent flow to mix and establish into well-defined freestream turbulence. Hot wire anemometry was used to quantify the level of turbulence in both the $\mathrm{x}$ and $y$ coordinates at the entrance of the diffuser. Using the various rod sets, Hoffman achieved various turbulence intensities ranging from $0.57 \%$ up to $3.07 \%$ in the streamwise direction and $0.57 \%$ to $8.91 \%$ in the y-direction. Turbulence intensity is defined

$$
T u=\frac{u_{r m s}}{U} \times 100 \%
$$

where $U$ is the mean streamwise velocity and $u_{r m s}$ is the RMS (root-mean-square) of the streamwise fluctuating velocity, $u^{\prime}$. The fluctuating velocity is defined as:

$$
u^{\prime}=u-U
$$

where $u$ is the streamwise velocity component. To obtain y-direction turbulence intensity, the RMS of the y-direction fluctuating velocity is also divided by the mean streamwise velocity. 


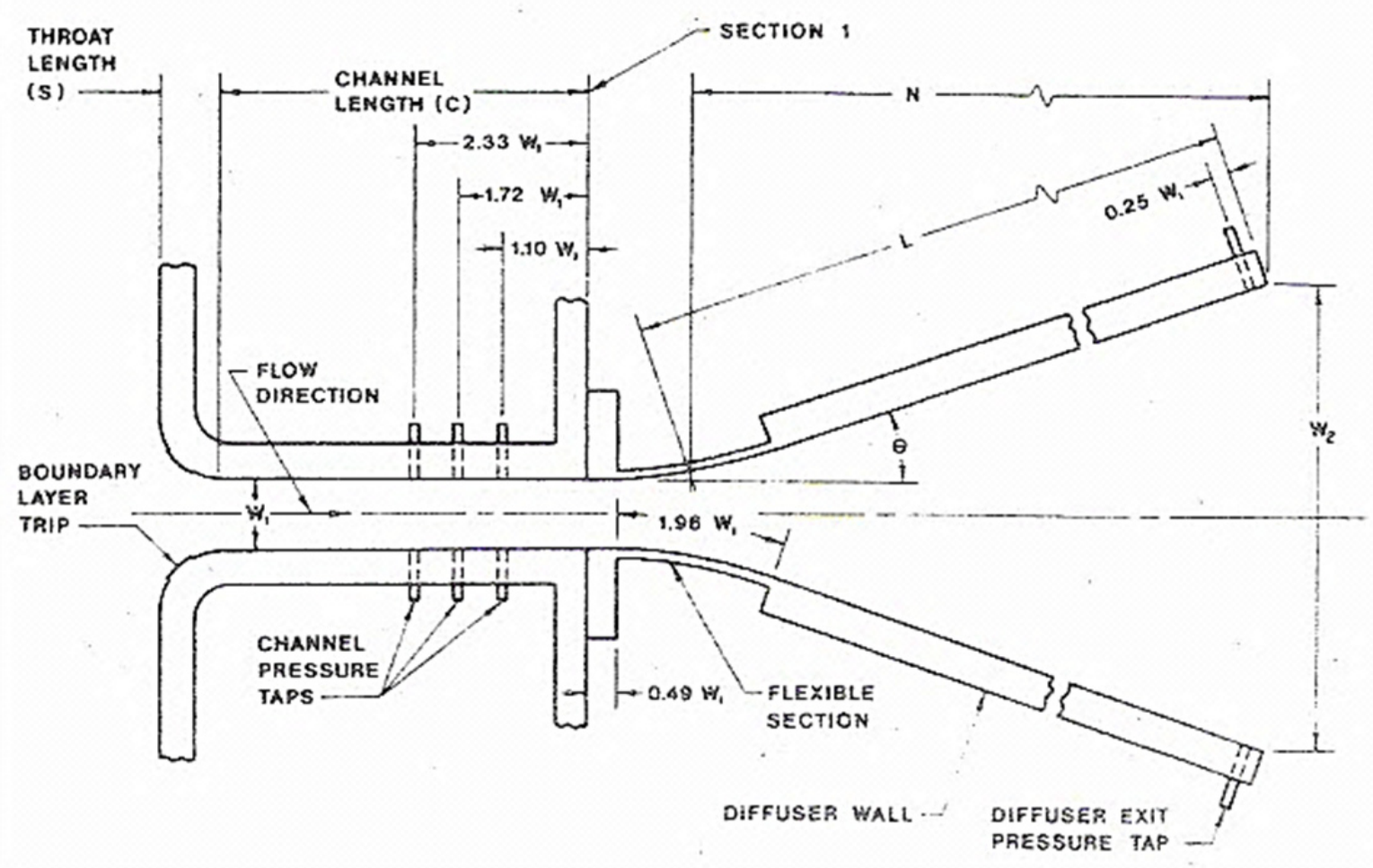

Figure 2.6: Schematic of inlet, channel and diffuser [13].

The divergence of the diffuser is adjustable allowing for varying area ratio. The rod sets were investigated with two different configurations, $2 \theta=12^{\circ}$ and $2 \theta=20^{\circ}$. As defined in Figure 2.6, $\theta$ is the divergence angle of one wall of the diffuser, and therefore $2 \theta$ is the total divergence angle of the diffuser. The baseline pressure recovery was much lower for $2 \theta=20^{\circ}$ than for $2 \theta=$ $12^{\circ}$ and hence the increase of pressure recovery was greater for the $2 \theta=20^{\circ}(23.9$ percent as opposed to 11.3 percent). Interestingly the highest pressure recovery achieved for the $2 \theta=20^{\circ}$ case was with a different rod set than for the $2 \theta=12^{\circ}$ case. 


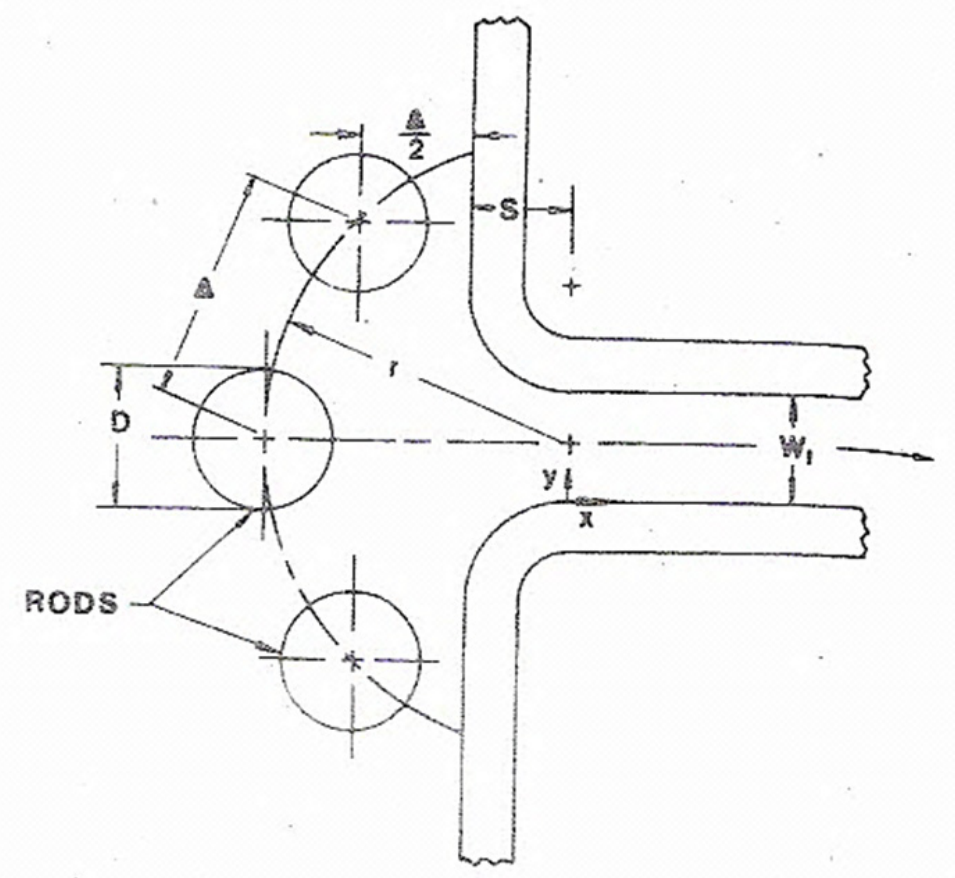

Figure 2.7: Schematic of rods used to generate turbulence [13].

The resulting data show a trend upward in pressure recovery with an increasing turbulence in the y-direction direction as shown in Figure 2.8. The data seem to indicate that there is a threshold of about $3 \%$ y-direction turbulence intensity before the pressure recovery is affected. It appears as though the pressure recovery is relatively insensitive to streamwise turbulence intensity, as seen in Figure 2.9, with the only correlation being attributable to its correlation with y-direction turbulence intensity. 


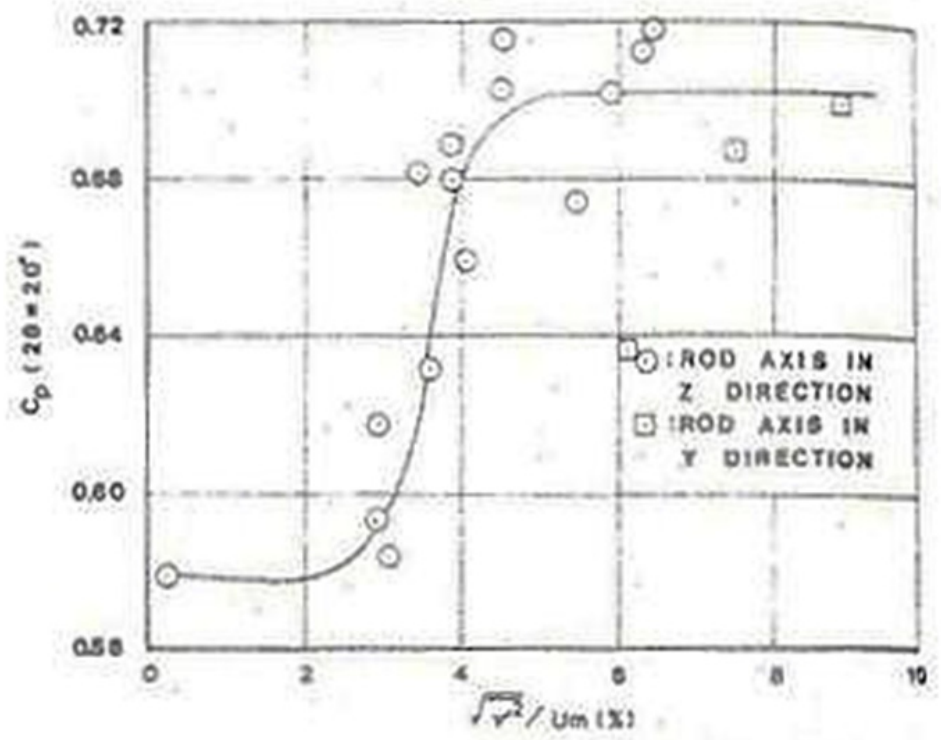

Figure 2.8: Pressure recovery coefficient as a function of y-direction turbulence intensity [13].

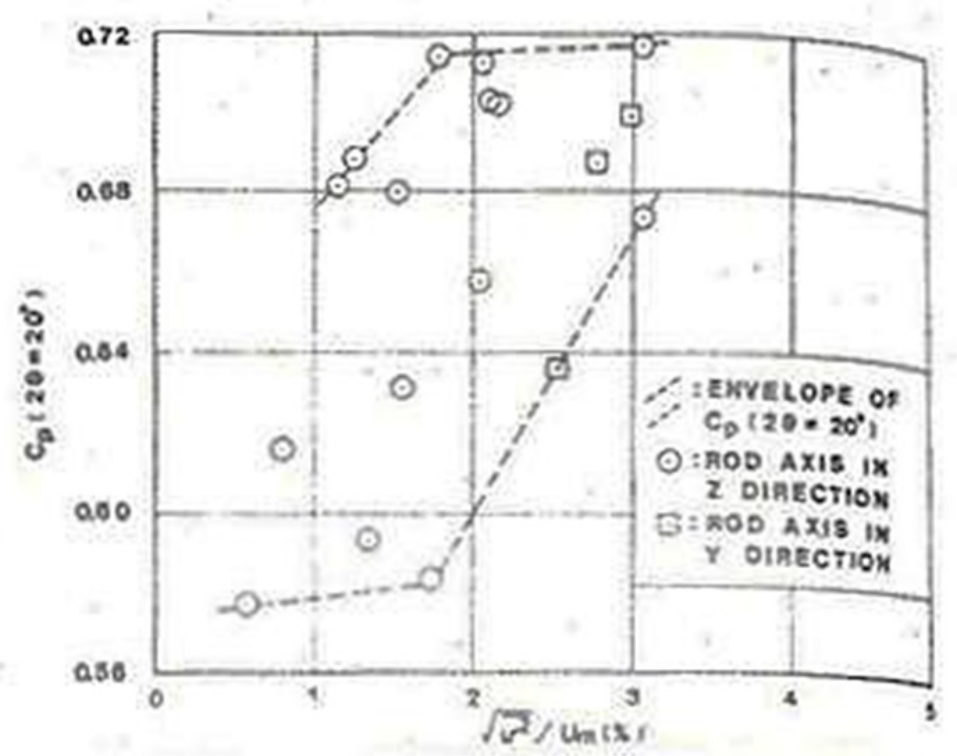

Figure 2.9: Pressure recovery coefficient as a function of streamwise turbulence intensity [13]. 
The study investigated the effect of varying the diffuser wall angle, or area ratio, on the diffuser pressure recovery. As expected, a consistent increase in pressure recovery with higher turbulence is seen for every value of area ratio. For these set of conditions, there appears to be an optimal area ratio for pressure recovery of approximately $3.4(2 \theta=10)$ which appears to be independent of the level of turbulence entering the diffuser. Consistent with the other results in this experiment, the gap in pressure recovery between the varying levels of turbulence widens as the area ratio, or diffuser angle, increases.

Hoffman also calculated the integral length scale, $\lambda_{x}$, for each data set and looked for correlation with pressure recovery. The integral length scale is an estimate of the breadth of the largest eddy of the turbulent flow. The characteristic size of turbulent eddies can have an impact on how turbulent flow behaves and interacts with its surroundings (see Appendix B for a more detailed description of turbulence length scales). It was observed that the highest pressure recovery cases had a $\lambda_{x} / \delta^{*}$ of 7.2 or higher, where $\delta^{*}$ is the boundary layer displacement thickness calculated from the measured velocity profile.

In summary, by delaying separation in the diffuser, increasing freestream turbulence increases static pressure recovery at the diffuser exit. The increase in pressure recovery is even greater for larger area ratios (or diffuser angles) where the baseline recovery is smaller due to more significant flow separation. The increase in pressure recovery appeared to be better correlated with the y-direction turbulence intensity than with the streamwise turbulence intensity. It is hypothesized that the y-direction turbulence more effectively transmits the freestream energy to the diverging walls in the diffuser. There was observed to be an optimal area ratio, or diffuser angle, to achieve pressure recovery. Although the increase in pressure recovery with increasing freestream turbulence becomes more significant as the area ratio increases. 
Hoffman's research provides a benchmark for this study. It shows that freestream turbulence has a net positive effect on pressure recovery of a diverging channel. This positive effect is achieved because the turbulent kinetic energy of the flow transmits to the walls and delays and reduces the flow separation that occurs due to divergence. Hoffman's study does not give an indication of how freestream turbulence will affect the flow separation that occurs due to bends in the duct such as in a serpentine diffuser, such as was investigated in the present study.

\subsection{Turbulence Generation}

In order to observe the effects of freestream turbulence on serpentine diffuser performance, it is necessary to determine a suitable method of generating turbulence in an experimental environment. Since the early days of fluid dynamics research, scientists have used various methods to generate freestream turbulence for experimental purposes. There are many distinct methods that have been used to generate turbulence.

The most basic method of turbulence generation that has been used is a coarse grid or screen of round or square bars [14]. Perforated plates [15], and streamwise tube bundles [16] have also been used in place of a grid of bars. A grid with active rotating vanes, first developed by Makita [17] and used by others [18], [19], generated considerably higher turbulence than a passive grid. Another type of active screen that has been used to generate higher levels of turbulence is a "mechanically agitated grid" [20]. More active methods that have been used include injecting a secondary flow into the main flow. In Thole et al. [21], air was injected into the mainstream flow of their experiment as cross-flow jets which produced higher levels of turbulence intensity than with any type of passive method. In some cases the combination of a passive and an active method was used as in the case of Tassa et al. [22] where air was injected 
into the main flow through a turbulence grid with several small circular holes distributed across the surface of bars.

After reviewing the different methods of turbulence generation it was concluded that a simple turbulence grid would suffice for the purposes of this study. A turbulence grid, in addition to being simple to fabricate and implement, is the most used and studied method. Because of this, there are many resources and tools available to help analyze and design a grid that would meet the needs of this study.

\subsubsection{Flow Through Screens}

Flow through turbulence grids, or screens, can be characterized as a special case of a free shear type flow. This is generally considered to be a good characterization everywhere except near the wall of the installation.

Initially, the flow physics behind each bar of the screen is identical to that of a plane wake. However, the flow physics changes drastically immediately after the wakes produced by the bars coalesce. The turbulent kinetic energy, $k$, of a flow is an indicator of the level of turbulence the flow is experiencing. The definition of $k$ is "the mean kinetic energy per unit mass in the fluctuating velocity field" [23]. Mathematically, $k$ is defined

$$
k=\frac{1}{2}\left(\overline{\left(u_{1}^{\prime}\right)^{2}}+\overline{\left(u_{2}^{\prime}\right)^{2}}+\overline{\left(u_{3}^{\prime}\right)^{2}}\right)
$$

where $u$ ' is the fluctuating velocity component defined

$$
\mathrm{u}^{\prime}=\mathrm{u}-\mathrm{U}
$$

where $u$ is the velocity component and $U$ is the mean velocity. The conservation equation for turbulent kinetic energy is written 


$$
\frac{\overline{\mathrm{D}} \mathrm{k}}{\overline{\mathrm{D}} \mathrm{t}}+\nabla \cdot \mathrm{T}^{\prime}=\mathcal{P}-\varepsilon
$$

where $T$ is the flux of energy (and $T$ is fluctuating $T$ ), $P$ is the production of turbulent kinetic energy, and $\varepsilon$ is the rate of dissipation of turbulent kinetic energy. For most free shear flows, such as the round jet and the plane wake, both the production, $P$, and the dissipation rate, $\varepsilon$, are non-zero. In the case of flow through a turbulence screen the production goes to zero upon the coalescing of each individual plane wake, leaving only the dissipation rate on the right side of the equation [23]. Hence, the turbulence decays with increasing distance downstream of where the wakes initially coalesce.

Frenkiel [24] developed a correlation based on experimental results to predict the turbulence intensity produced by a particular turbulence screen. Roach [15] simplifies this correlation by eliminating the need to determine a virtual origin, although the correlation is then only good for the flow downstream of the coalescence of the plane wakes that the screen produces. The correlation presented by Roach is

$$
\mathrm{Tu}=\mathrm{C}\left(\frac{\mathrm{x}}{\mathrm{d}}\right)^{-\frac{5}{7}}
$$

where $T u$ is the turbulence intensity, $x$ is the distance downstream of the screen, $d$ is the bar diameter and the constant denoted by $C$ is dependent mostly on the grid geometry, although the Reynolds number beyond a certain threshold will have a minor effect. Roach tabulates values of $C$ for the different types of screens that are commonly used, as well as provides a formula to determine an equivalent $d$ for a perforated plate. Roach's correlation has been shown to be considerably accurate when compared to experimental data such as a study performed by McQuilling [25]. 


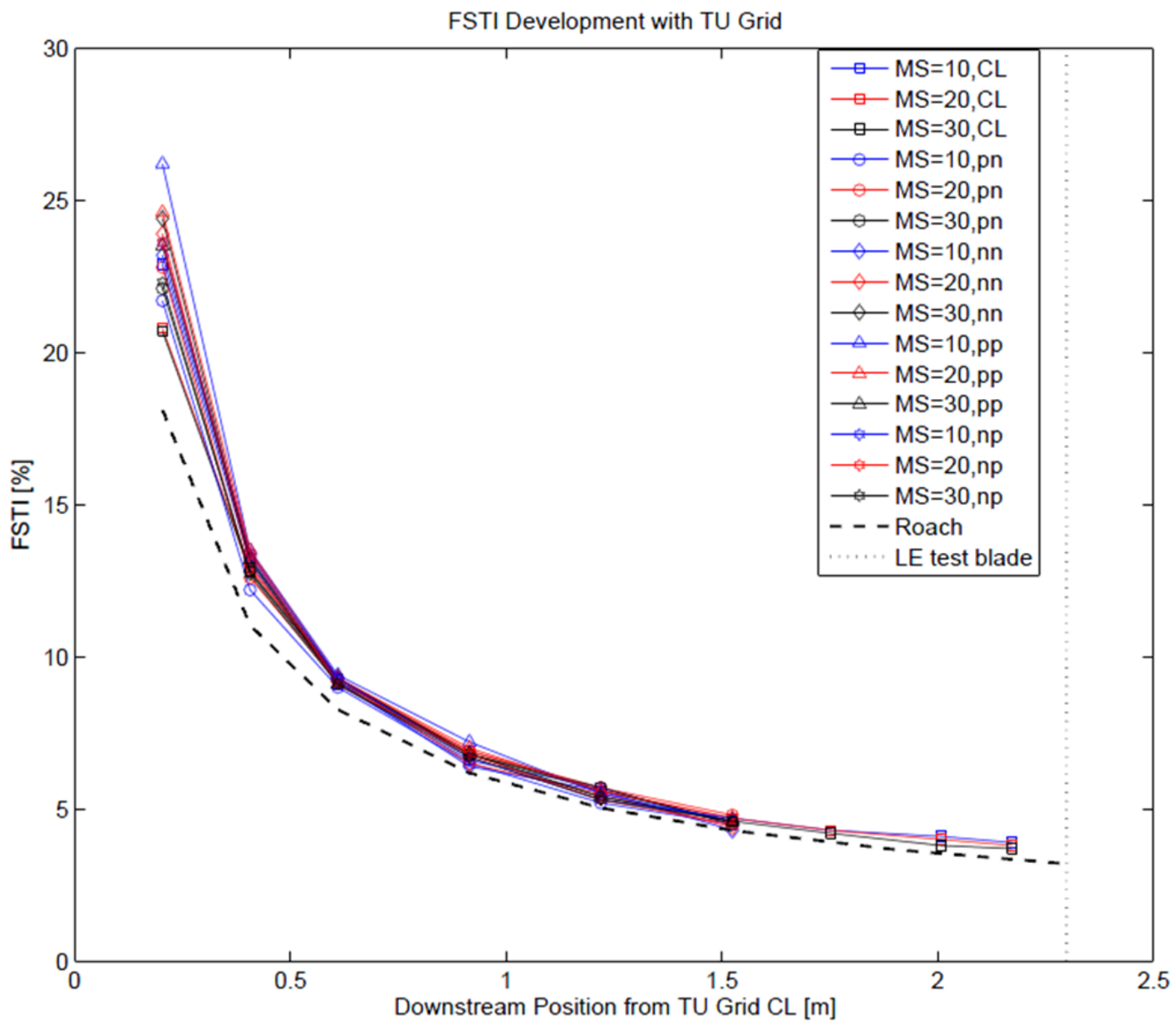

Figure 2.10: Turbulence behavior downstream of turbulence grid at various cross-sectional positions compared with the correlation by Roach [25].

In this study, McQuilling investigated the turbulence development in a low-speed wind tunnel prior to the flow reaching the test article. He takes measurements axially along the wind tunnel downstream of the turbulence screen at various points across the cross-section of the tunnel and at varying motor speed. The measured freestream turbulence intensity is plotted as a function of the distance downstream of the turbulence grid as shown in Figure 2.10. LE test blade refers to the leading edge of the test blade located inside of the wind tunnel, and MS=10 refers to the motor speed and CL to the cross-sectional position of the measurement. 
Additionally, Roach [15] provides a correlation to predict the pressure loss across a turbulence screen. The pressure loss correlation is

$$
\frac{\nabla p}{q}=A\left(\frac{1}{\beta^{2}}-1\right)^{B}
$$

where $p$ is the pressure loss, $q$ is the upstream dynamic pressure, $\beta$ is the grid porosity, and $A$ and $B$ are coefficients that are functions of the grid geometry for incompressible flow.

For more detailed information, analyses and discussions on the properties of flow through screens including energy, harmonics and scales see [14], [15], [26], [27], and [28]. 


\section{CHAPTER 3. COMPUTATIONAL ANALYSIS}

In this chapter, a Computation Fluid Dynamics (CFD) analysis for this will be presented and discussed. The CFD analysis was performed by Dr. Darius Sanders, who is also a member of the Fan and Compressor Branch of the AFRL. This analysis was performed to assist the study by providing a preliminary look at the effect of freestream turbulence on the distortion patterns at the AIP of a serpentine diffuser. The distortion patterns that were investigated are the total pressure distortion and swirl distortion patterns. The results of this analysis were influential in the development of the test procedure for the present study.

\subsection{CFD Parameters}

The CFD analysis was performed using the commercial code STARCCM+ v6.02. The entire flow barrel and model serpentine diffuser were modeled with a mesh of $6,815,745$ polyhedral cells. The $k-\omega S S T$ turbulence model was used to resolve the steady state solution. Two distinct configurations were analyzed with CFD. In one of the cases, the inlet starts at the entrance to the flow barrel of the facility. In the other case, the inlet starts at the face of the mouth of the diffuser. In each case, an inlet turbulence intensity of $2 \%$ is compared with an inlet turbulence intensity of $10 \%$. 


\subsection{CFD Results}

In the first case, where the turbulence intensity was set at the inlet to the flow barrel, the turbulence can be seen to entirely decay before the flow reaches the mouth of the diffuser. This is demonstrated in Figure 3.1, which is a plot of the turbulence intensity in the flow barrel leading up to the diffuser mouth for each case. The distance and the acceleration of the flow cause the turbulent energy to dissipate as the air makes its way through the flow barrel and into the diffuser.

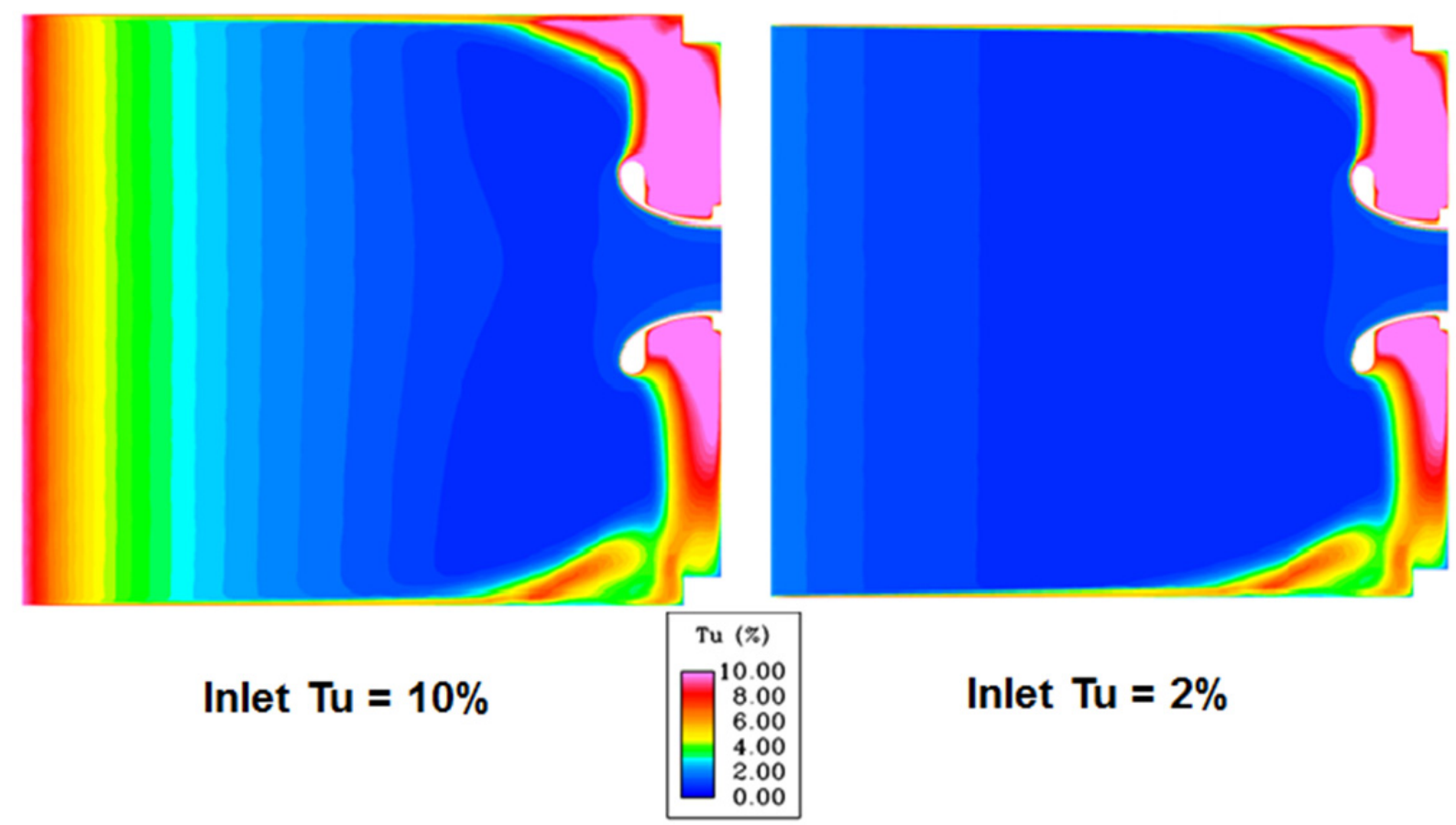

Figure 3.1: Turbulence intensity field for two inlet turbulence intensities with turbulence level set at flow barrel inlet.

Because the turbulence entirely decayed before reaching mouth of the diffuser there was no difference in the distortion patterns seen at the AIP. This information was instrumental in putting together a test plan for the experimental research of this study. It was determined that it 
would be desirable to place the turbulence generator as close to the mouth of the diffuser as possible. Although, because the turbulence generator used in this study is a square mesh grid, it was necessary to leave enough space for the wakes behind each bar to coalesce and form a welldefined turbulent flow.

In the second CFD test case, the turbulence was defined at the inlet of the diffuser. In order to facilitate this, a mesh of just the diffuser was used. The turbulence levels at the face of the diffuser mouth are shown in Figure 3.2.
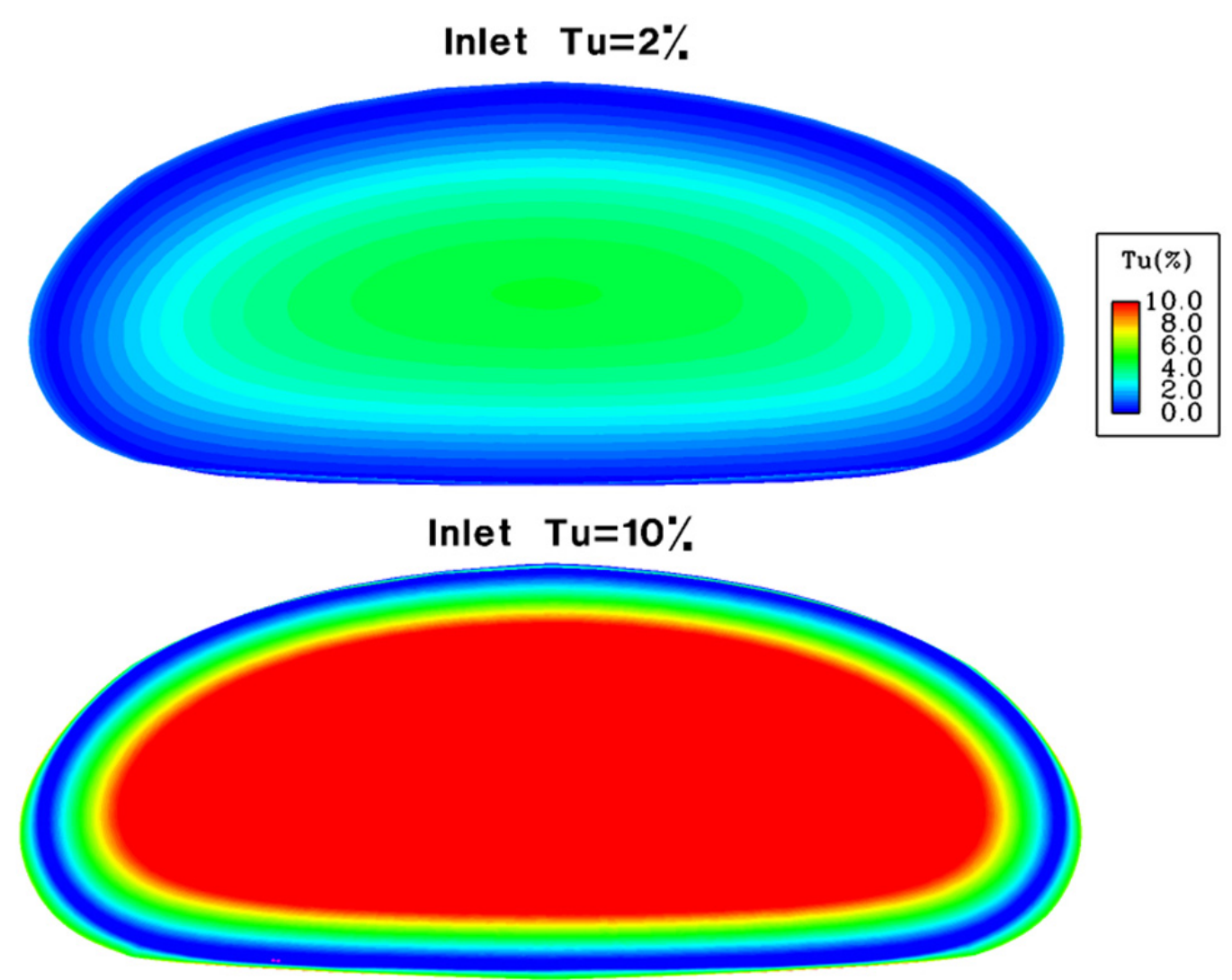

Figure 3.2: The turbulence intensity boundary conditions for two inlet turbulence intensities at the face of the diffuser mouth.

It was not expected at the time of this analysis that the turbulence screens used in this experiment would be able to achieve a turbulence intensity of $10 \%$ at the face of the diffuser 
mouth. The turbulence was set this high to ensure, if there were differences in the distortion patterns caused by freestream turbulence, that they would be noticeable. In this CFD analysis, the inlet boundary condition was allowed to be changed due to backflow as the mathematical turbulence model was calculated for the flow in the diffuser. An important, even crucial perhaps, observation is that the turbulence model did not allow for turbulence to exist near the wall. Note that even a turbulence of $2 \%$ at the diffuser mouth is slightly higher than in the previous case where the turbulence was set at the flow barrel inlet. The results for the distortion patterns with the turbulence levels fixed at the face of the diffuser mouth are presented in Figure 3.3.

\subsection{Discussion of CFD Results}

The total pressure deficit near the top of the AIP is caused by flow separation on the upper surface of the serpentine diffuser as the flow comes around the final turn (see Figure 2.3). This total pressure deficit poses the most serious threat to compressor performance and the reduction of its stability margin. From the results in Figure 3.3 it appears that freestream turbulence had no significant effect on the flow separation on the upper surface of the diffuser.

It is hypothesized that the $k-\omega S S T$ turbulence model may have difficulties analyzing the phenomena discovered by Hoffman [7] where the y-direction turbulence transmits the kinetic energy of the flow to the walls, reducing flow separation. It can be seen in Figure 3.2 that the model drives the level of turbulence at the wall to zero, and this could potentially lead to a failure in predicting the effect of turbulence on that flow separation that occurs on the upper surface of a serpentine diffuser. 

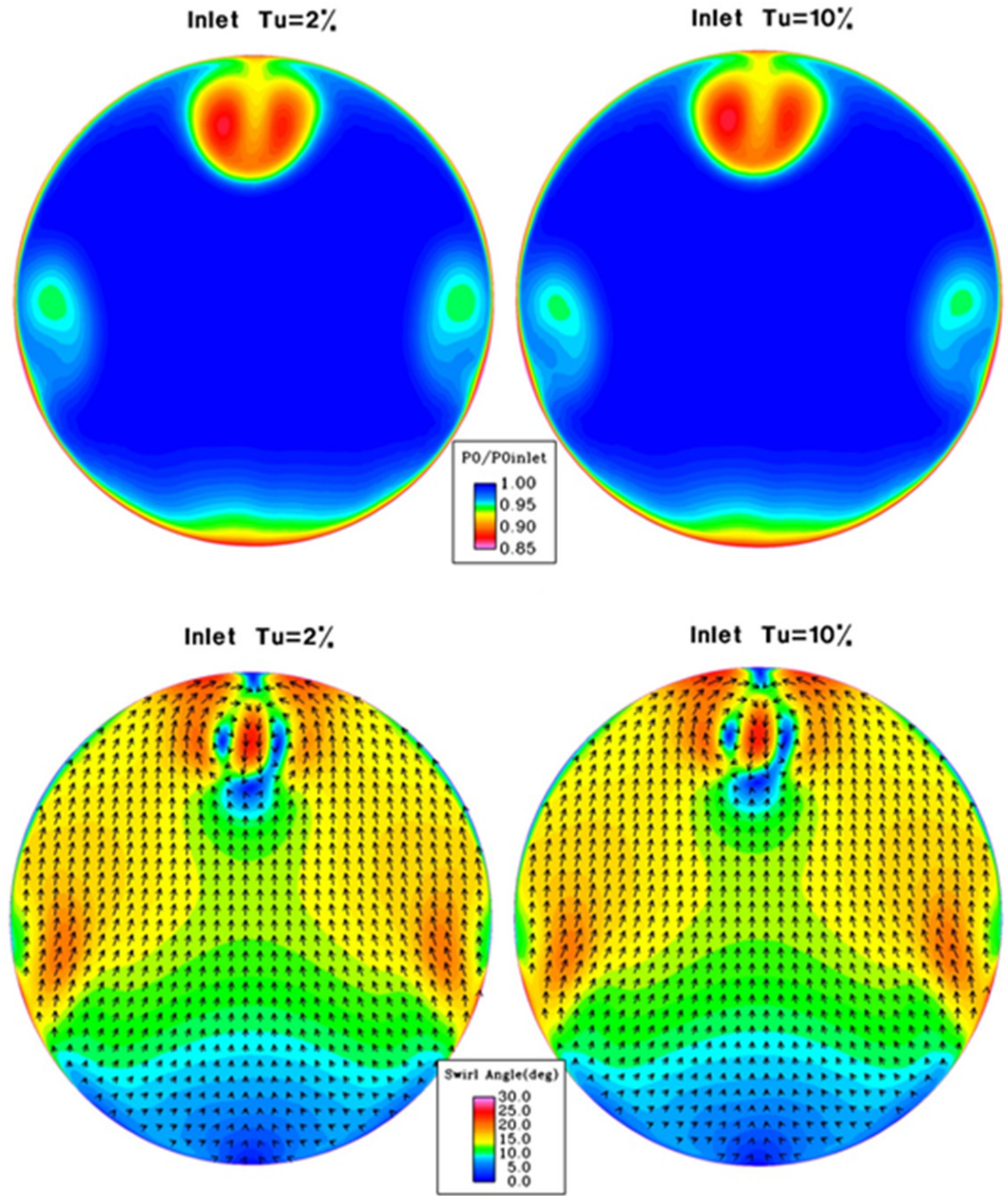

Figure 3.3: Total pressure and swirl distortion patterns at the AIP for each turbulence level with the turbulence specified at the face of the diffuser mouth. 
The other notable characteristics of the AIP pressure recovery of a serpentine diffuser are the side wall vortices and the boundary layer development on the bottom. There is a slightly noticeable reduction in the side wall vortex development, indicated by a decrease in the total pressure drop, between the baseline and the elevated freestream turbulence cases. The boundary layer development along the lower surface appears identical between each case. There seems to be no difference in swirl distortion either. 


\section{CHAPTER 4. METHOD AND EXPERIMENTAL SETUP}

This chapter describes in detail the facility and equipment that were used to perform this work. The experimental procedure that was followed to obtain data will also be described.

\subsection{Research Facility}

The Fan \& Compressor branch of the AFRL operates a research laboratory known as the Compressor Aero Research Laboratory (CARL). The CARL comprises two separate test facilities, the compressor facility and the annular cascade facility (see Figure 4.1), and a soundisolated control room. The control room houses the workstations, data servers, A/D units, etc. that are used to interface with the facility equipment. The CARL is home to where much of the HIT research is taking place. The schematic of the CARL in Figure 4.1 shows a model serpentine diffuser installed atop the annular cascade facility. The annular cascade facility was used to perform this study. More details about the annular cascade facility, the model serpentine diffuser and the hardware housed in the facility will be described in this section. The schematic also shows the model diffuser installed with the compressor facility which will be implemented to study the effects of serpentine diffuser induced flow distortion on compressor performance for other HIT investigations, but the compressor facility was not used in this study. 


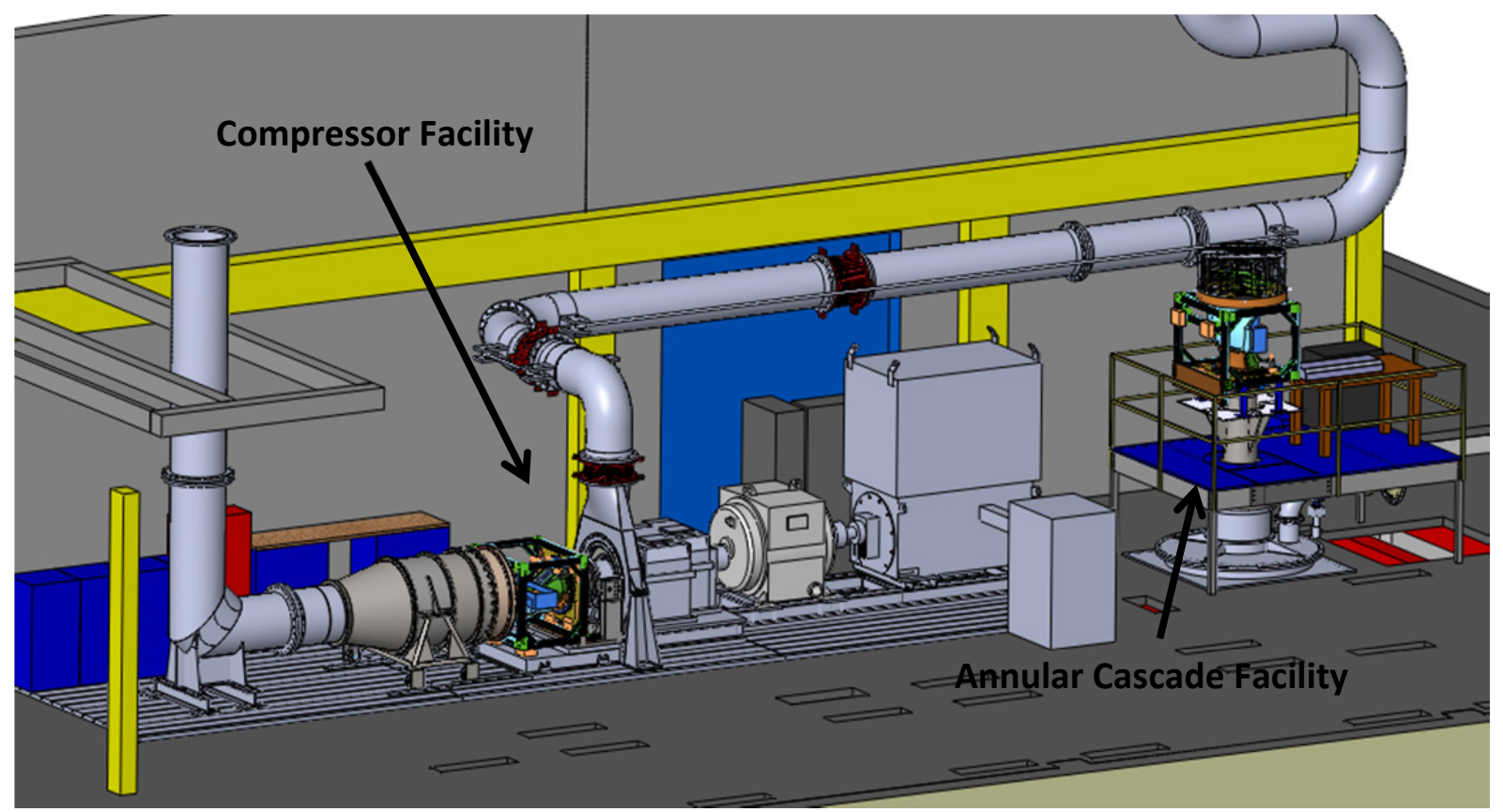

Figure 4.1: Schematic of the CARL Facility.

\subsubsection{Annular Cascade Facility}

Located inside the CARL is the annular cascade facility (ACF). The name "annular cascade" refers to an annular cascade of static compressor rotors and stators installed in the facility. However, they are not installed for this study. In these experiments the ACF is used to perform isolated diffuser tests. The ACF pulls air vertically downward, hence the diffuser is positioned upright and air is pulled in from above. The ACF is powered by large industrial exhausters with the ability to pull over $100 \mathrm{lb}_{\mathrm{m}} / \mathrm{s}$. In Figure 4.2, the base of the ACF, without the diffuser installed, is shown with some of its individual components.

Currently, the ACF is being used to generate test conditions for a model serpentine diffuser. Attached to the ACF downstream of the model diffuser is a trim station. The trim station is a separate inlet with a valve that is electronically controlled with a step motor (see Figure 4.2). When open, air is pulled in through the trim station by the exhausters, bypassing the test article. It is used to adjust the flow rate through the model diffuser to achieve the desired 
Mach number at the diffuser throat. The flow conditioning pipe bounds the flow well beyond the AIP to prevent installation effects from disturbing the measurements. The test article, which in this case is the model diffuser, is installed on the inlet duct.

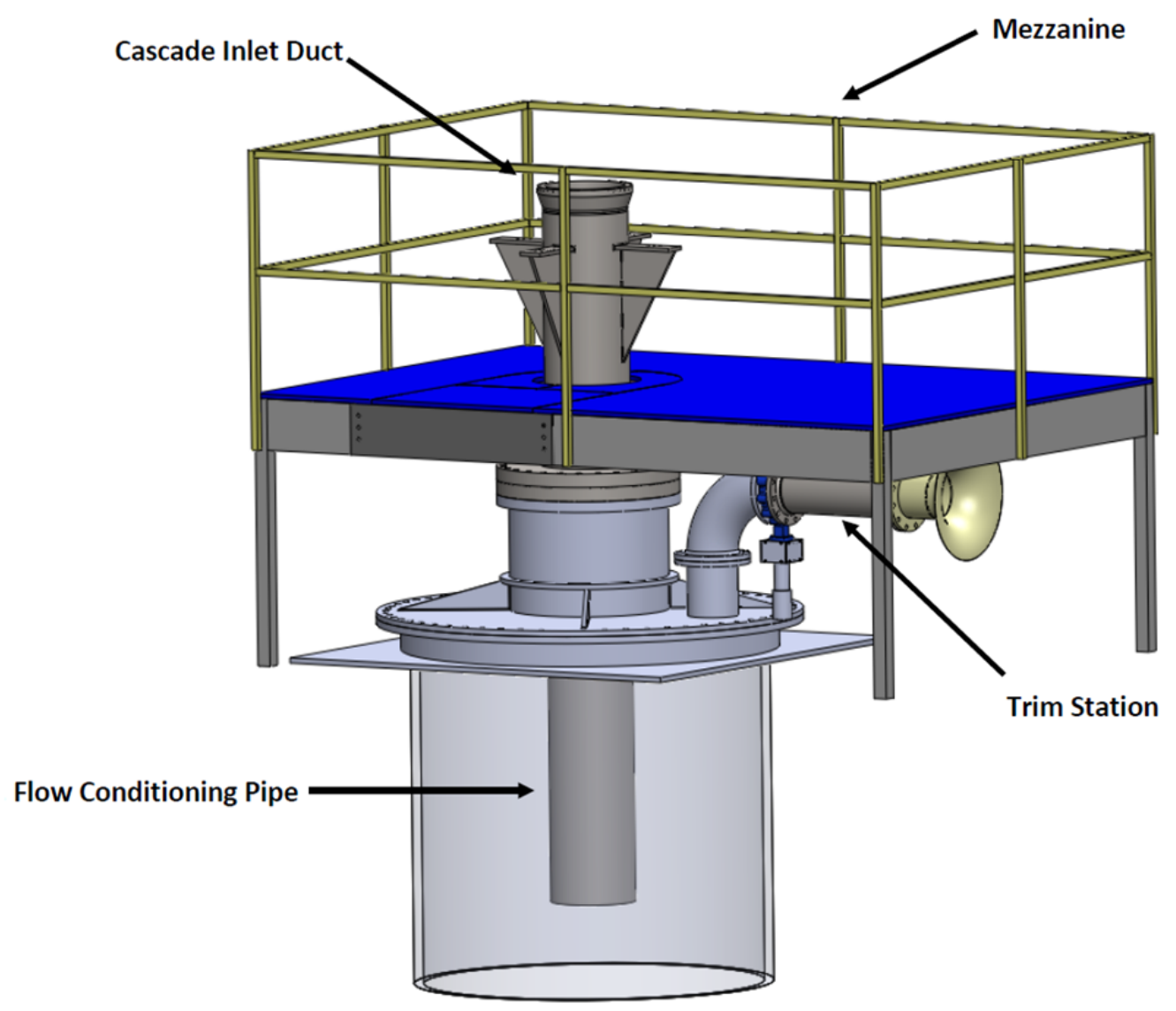

Figure 4.2: The ACF Facility.

\subsubsection{Serpentine Diffuser Test Model \& Axial Static Pressure Measurement}

The model of the serpentine diffuser was manufactured by Tri-Models, Inc. It features several static pressure taps along the non-linear axis of the diffuser. It also features static pressure taps circumferentially about the inlet and outlet planes as well as two additional cross sections inside the diffuser, although these pressure taps are not used in this experiment. 
Specifically, there are 50 pressure taps along the bottom surface and 46 taps along the upper surface that were used in this experiment. The location of these pressure taps are tabulated in Appendix E. The pressure taps of particular interest are the taps along the upper surface between $\mathrm{x} / \mathrm{L}=0.451$ and $\mathrm{x} / \mathrm{L}=0.602$ (see Figure 4.3) which were used to determine the effect of freestream turbulence on the separation that occurs along the diverging wall. The pressure tap readings are measured using Scanivalve DSA 3016 pressure models with a full scale range of 5 psi. A total of 9600 samples were taken and averaged to complete each data point.

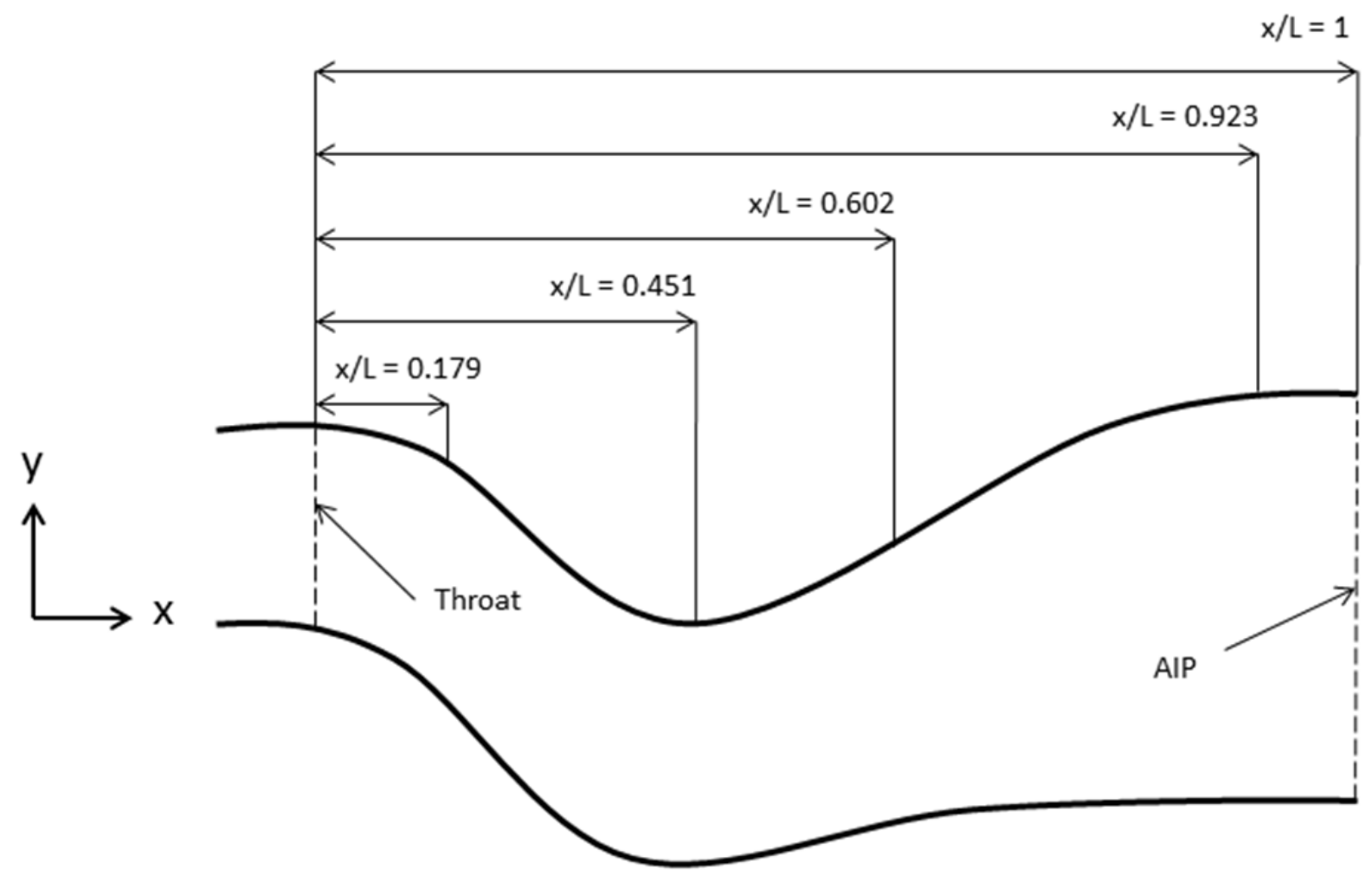

Figure 4.3: Normalized distance of certain locations along the axis of the serpentine diffuser (Note: profile of image not proportional to diffuser used in this experiment).

The model diffuser has the ability to rotate about its axis independent of the hardware that is fastened above and below it. This allows for variation of the angle at which measurements are taken at the Aerodynamic Interface Plane (AIP) which is located at the exit of 
the diffuser. Its rotation is driven by an electric motor and measured by a potentiometer which can specify the azimuthal position to within $0.1^{\circ}$.

\subsubsection{Flow Barrel}

Assembled to the top of the model diffuser, shown in Figure 4.4, is a flow barrel to create a uniform flow upstream of the diffuser (also see Figure 4.11). The flow barrel consists of sections which allows for configurability such as the insertion of a turbulence screen. The initial section is a converging "bellmouth" where the air enters. The bellmouth inlet to the flow barrel creates a contraction of the air flow which helps to reduce non-uniformities inherent in the flow.

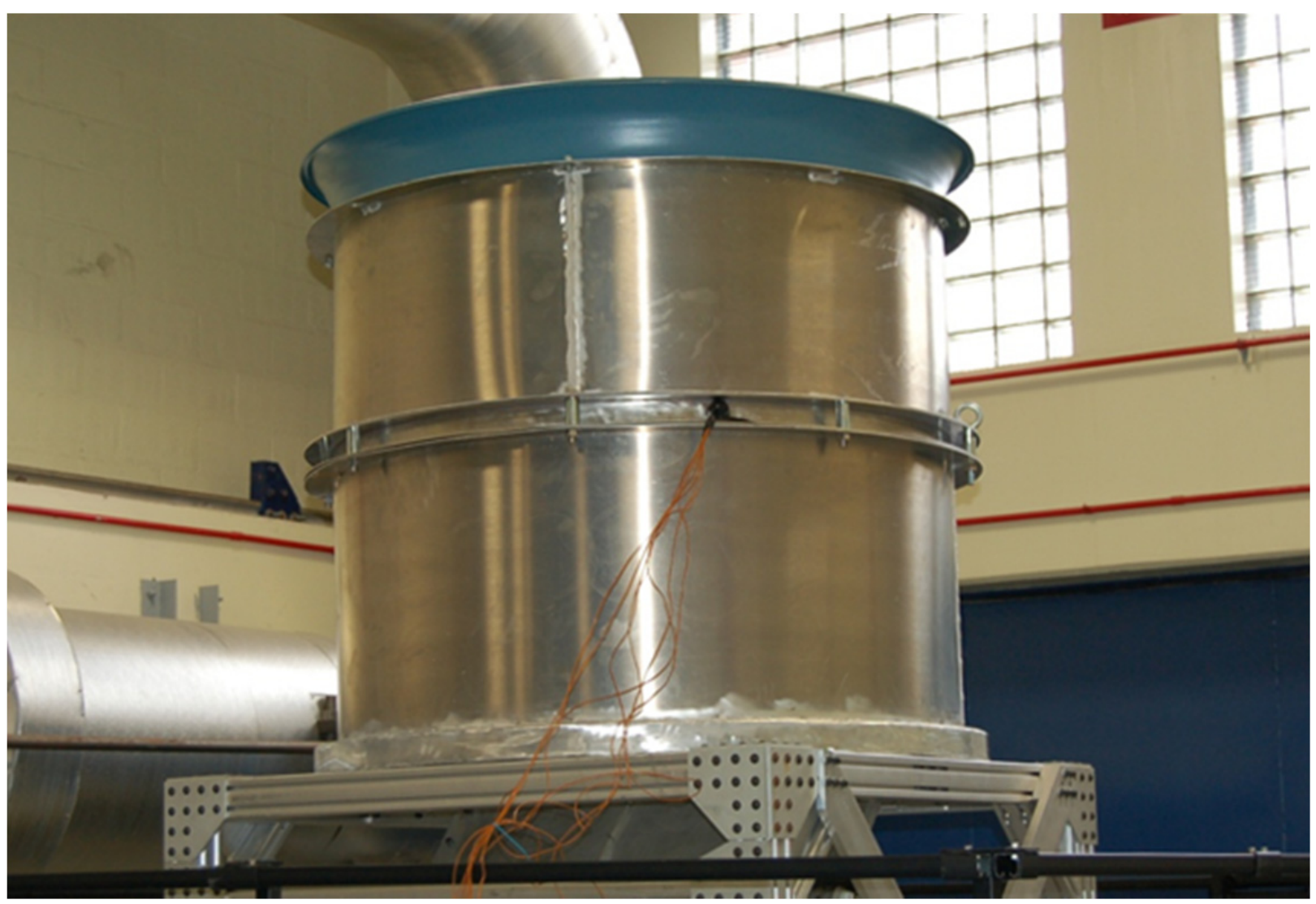

Figure 4.4: Flow barrel installation on model diffuser of ACF. 


\subsubsection{Velocity Measurement System}

The velocity measurement technique that was used to define the turbulence entering into the diffuser is constant temperature anemometry (CTA) with hot film probes (also known as hot wire anemometry). The CTA component used in this experiment is the IFA-300 unit designed and manufactured by TSI Inc. The hot film probes were also manufactured by TSI Inc. The probes were calibrated using a Model 1127 TSI Air Velocity Calibrator (see Appendix A for more information on the calibration procedure).

The IFA-300 includes internal signal conditioning that can be applied to the output voltage which is directed to the A/D board for computer processing. The settings of the IFA-300 were maintained constant from calibration to experiment.

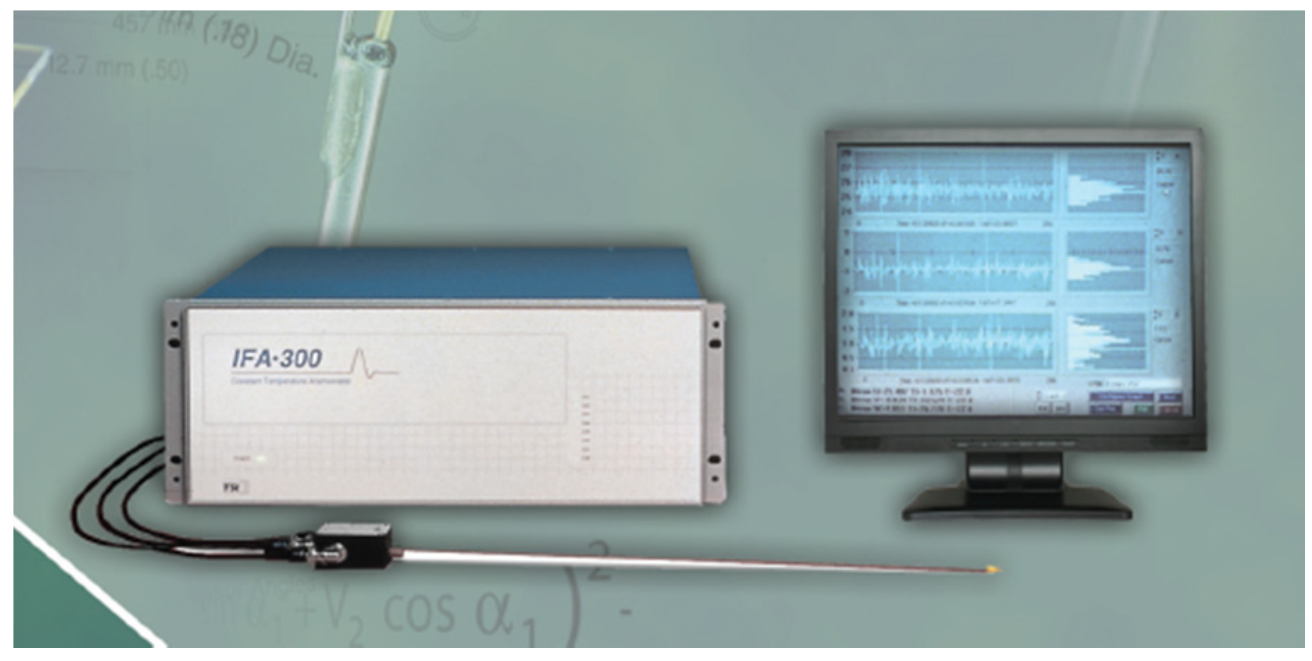

Figure 4.5: IFA-300 Constant Temperature Anemometer [29].

\subsubsection{AIP Pressure Measurement}

In order to measure the total pressure field at the AIP a rake case was installed immediately after the diffuser (see Figure 4.6). The rake case houses 8 pitot tubes rakes with 10 pitot tubes per rake for a total of 80 pitot probes to measure the planar profile of the total 
pressure distortion at the AIP. The spacing of the pitot probes was designed such that each probe represents a section of equal area. The pitot rakes are also evenly spaced circumferentially around the case resulting in a $45^{\circ}$ angle between each rake. The number and spacing of the rakes were determined based on recommended practices [4].

With the ability of the model diffuser to rotate independently of the rake case it is possible to take pressure measurements at smaller increments than $45^{\circ}$. In this study, the diffuser was rotated in $5^{\circ}$ increments resulting in 720 total pressure measurements at the AIP for each case. The location of each total pressure measurement across the AIP is demonstrated by the line contours in Figure 4.7. Using the 720 measurements, a color-gradient contour plot of the total pressure can be interpolated for the entire AIP. The pitot probes pressure readings are also measured using the Scanivalve pressure transducer with 9600 samples taken per point. A total pressure reading at the inlet is used to correct the AIP total pressure readings to account for inlet variations. The contour plots are generated using TecPlot.

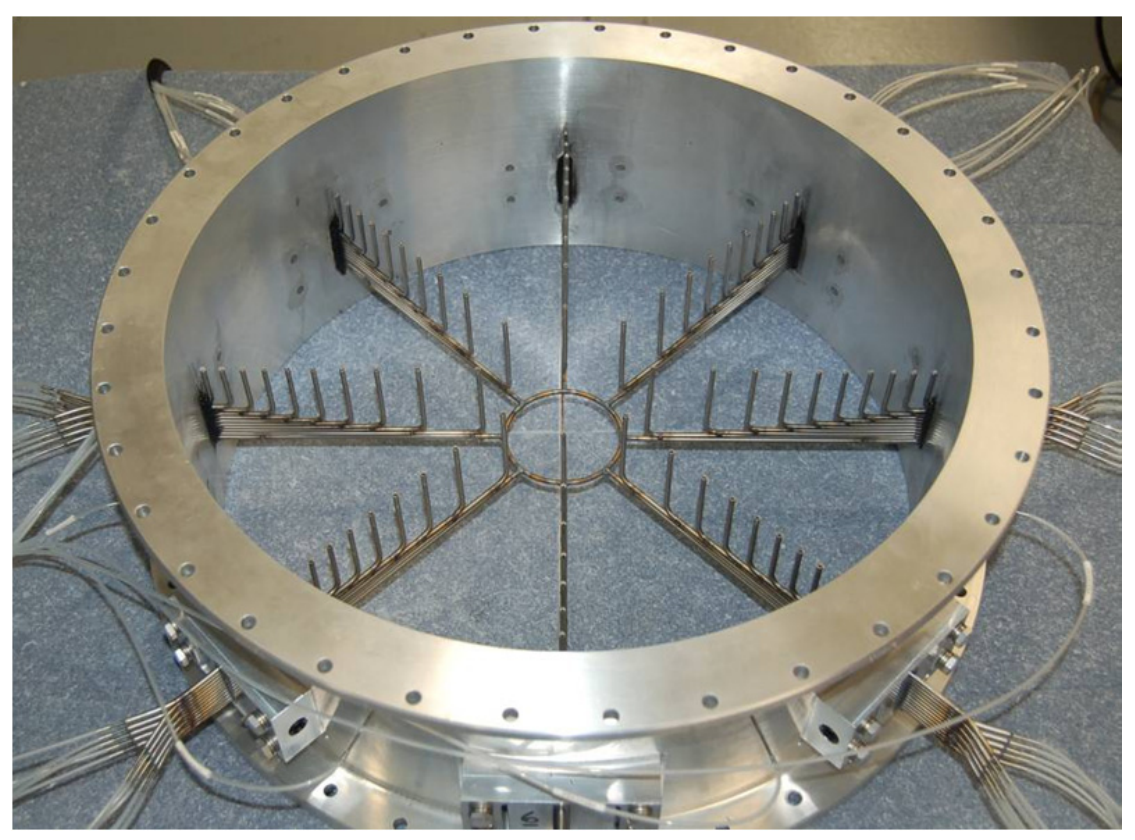

Figure 4.6: Steady Total Pressure AIP Rake Case. 


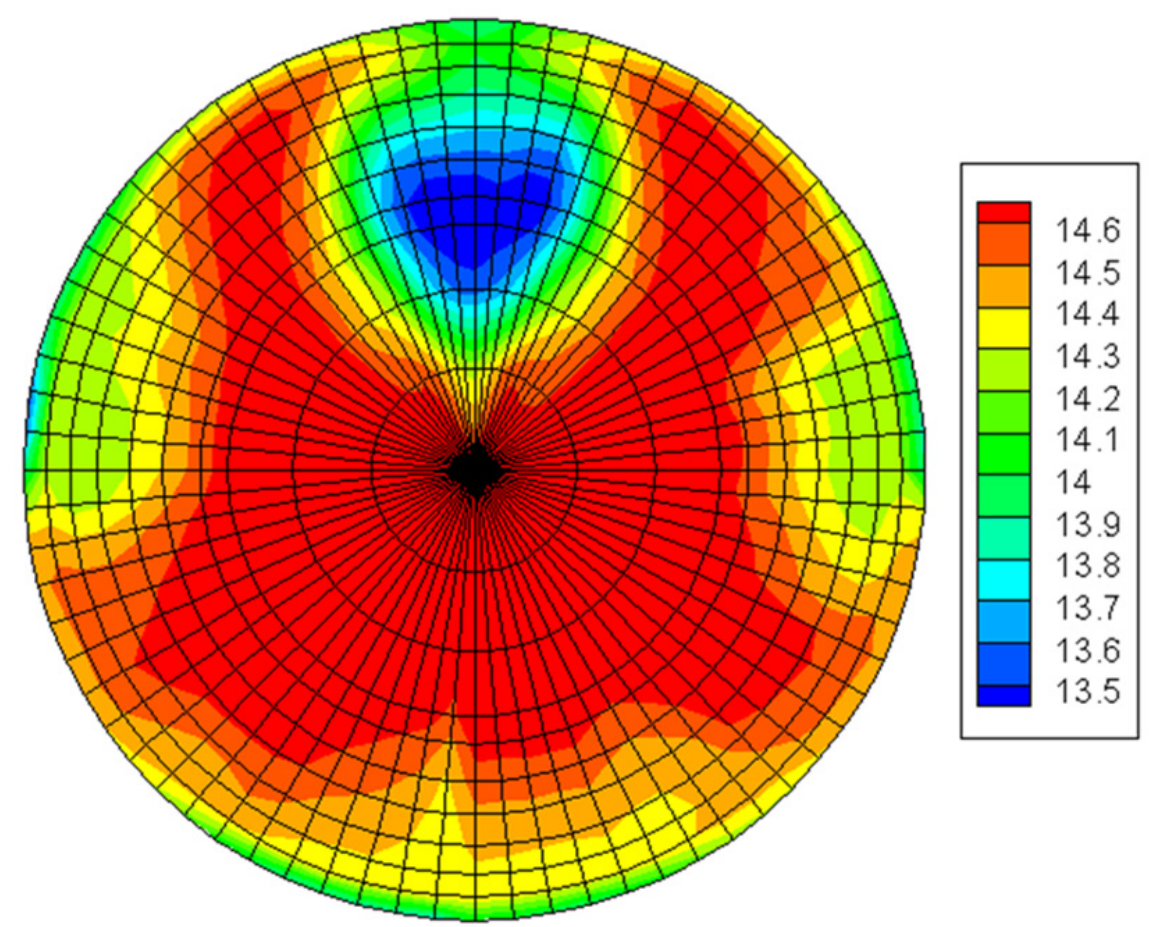

Figure 4.7: Contour of total pressure probe locations at AIP with sample total pressure field (psi).

\subsubsection{Turbulence Grids}

Two different turbulence grids were designed for this study. One was made of square bars and the other was made of round bars. Apart from the cross-sectional shape of the bars, the dimensions of the two turbulence grids are the same. For both screens, the bar diameter, $d$, is 0.5 " and the mesh spacing, $M$ (center to center distance between bars), is 2 ". The grid bars were welded together and to the inside of a frame with flanges that mate with the flow barrel assembly (see Figure 4.11).

Using Roach's correlations (see eqns. $2.6 \& 2.7$ ) [15], the turbulence intensity produced by these screens at key locations (see section 4.2.1), as well as the pressure losses, can be predicted. The predicted turbulence intensity generated by the square bar grid was $8.1 \%$ turbulence at 20 " downstream of the grid, or $9.6 \%$ at 15.7 " downstream of the grid. For the round bar, the predicted turbulence at 20 " downstream was $5.7 \%$ turbulence, and $6.2 \%$ at 18 " 
downstream (see Table 4.1). The predicted pressure loss across the square bar grid was approximately $0.12 \mathrm{psi}$. For the round bar grid, the predicted pressure loss was approximately 0.058 psi. It is important to note that the total pressure prove used to correct AIP total pressure readings at the inlet was located downstream of where the turbulence grids were installed. Hence, the AIP total pressure measurements are not affected by the pressure losses due to the grid or grids.

\subsection{Test Setup \& Procedure}

This section details the different test configurations that were studied, the hardware setup of the test equipment and the measurement procedure that was used.

\subsubsection{Test Configurations}

The first test configuration is the baseline case. This is the case where no turbulence screen is installed in the ACF. Sometimes it is referred to as the background turbulence case. Each of the remaining configurations is compared to the baseline configuration to quantify the effect that freestream turbulence has on serpentine diffuser performance.

In the second test configuration, the square bar turbulence grid was installed at the location $d=15.7$ " upstream of the mouth of the diffuser. The distance, $d$, is measured from the edge of the diffuser lip to the center of the square bar arrangement in the grid. This configuration is referred to as the single screen configuration.

In the third test configuration, both of the turbulence grids were installed. The square bar grid is in the same location as in the second test configuration. The round bar grid is installed directly upstream of the square bar grid at $\mathrm{d}=18$ ", with a center to center distance of 2.3 " 
between the grids. The round bar grid was intentionally oriented in a position such that the round bars are misaligned from the square bars so that the square bars were not located directly in the wake of the round bars. It was hypothesized that this test configuration would produce a higher level of turbulence than the second configuration with just the square bar grid. This configuration is referred to as the dual screens configuration.

In the fourth and final test configuration, the square bar turbulence grid was installed at the location $d=37.3$ " upstream of the mouth of the diffuser. The purpose for this test configuration was to observe the rise in turbulence generated by the square bar turbulence grid before the potential effects of the flow entering the diffuser are dominating the flow physics. This configuration is referred to as the high screen configuration.

The details that differentiate these test configurations are summarized in Table 4.1. Additionally, figures of the flow barrel installation for each test configuration are displayed in Figure 4.8.

Table 4.1: Test configuration summary.

\begin{tabular}{ccccc}
\hline Test Configuration & $\mathbf{1}$ & $\mathbf{2}$ & $\mathbf{3}$ & $\mathbf{4}$ \\
\hline Reference Name & Baseline & Single screen & Dual screens & High screen \\
Square Bar Grid Location & - & $\mathrm{d}=15.7 ”$ & $\mathrm{~d}=15.7 ”$ & $\mathrm{~d}=37.3 ”$ \\
Round Bar Grid Location & - & - & $\mathrm{d}=18 \%$ & - \\
Measurement Location & $\mathrm{d}=0.75 \%$ & $\mathrm{~d}=0.75 ”$ & $\mathrm{~d}=0.75 ”$ & $\mathrm{~d}=0.75 ”, 15.7 ”$ \\
\hline
\end{tabular}

For each of these test configurations the Mach number at the throat of the diffuser was set to the design value of 0.691 (within \pm 0.002 ). Additionally, for the baseline and the single screen configurations, the data were taken at seven different off-design Mach numbers ranging from 
0.55 to 0.725 . In these off-design Mach number conditions, the diffuser was not rotated and therefore only 80 AIP total pressure data points were taken for each Mach number.

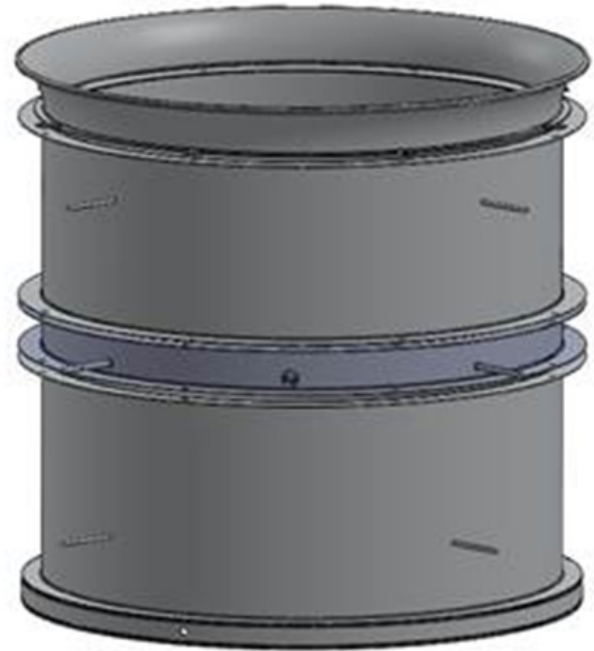

Baseline

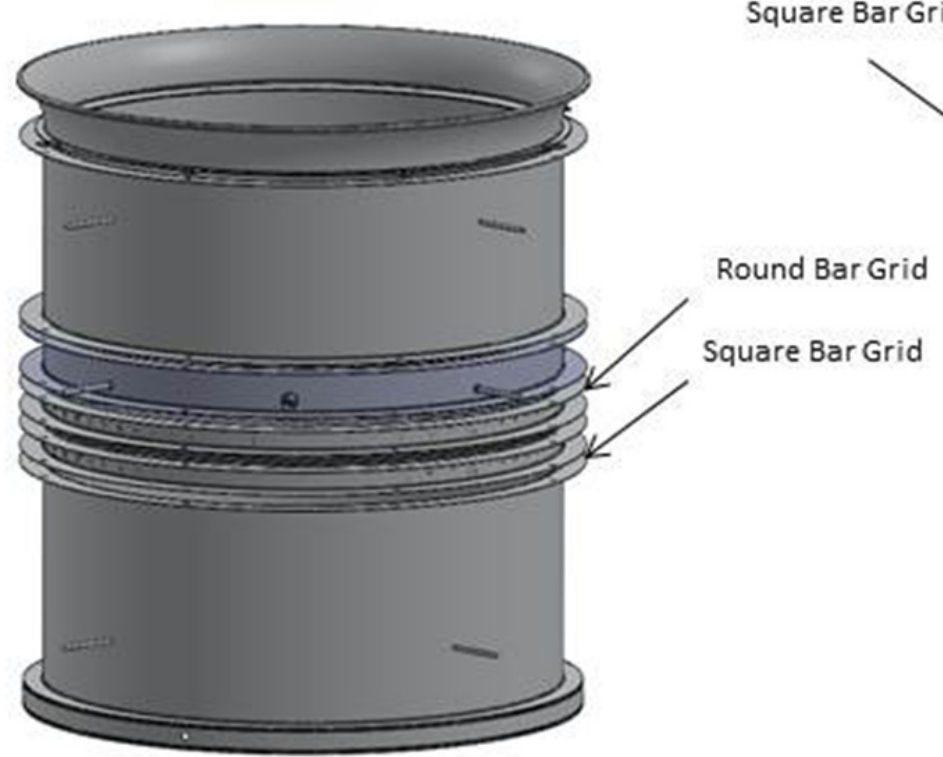

Dual Screens

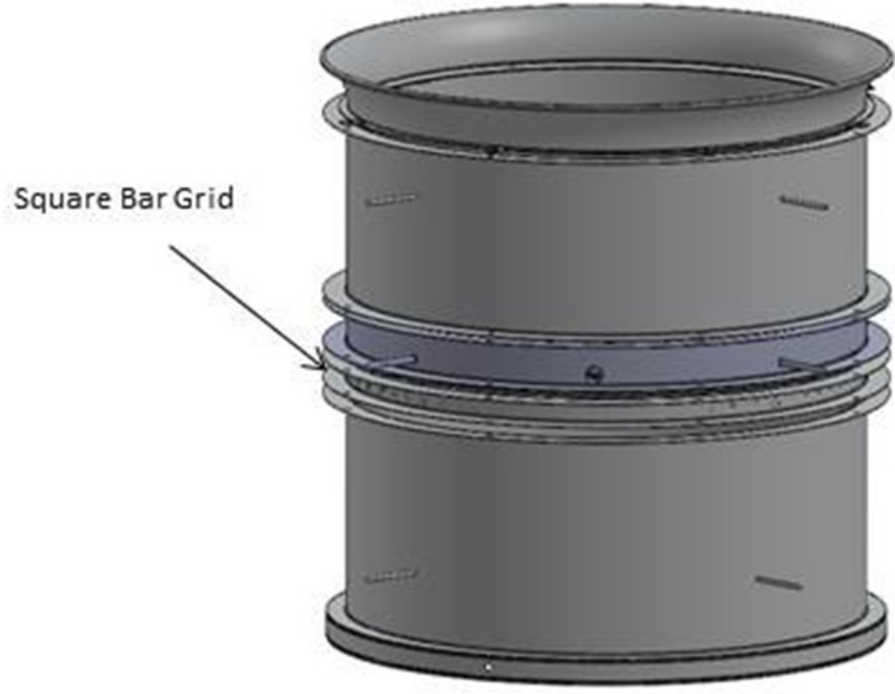

Single Screen

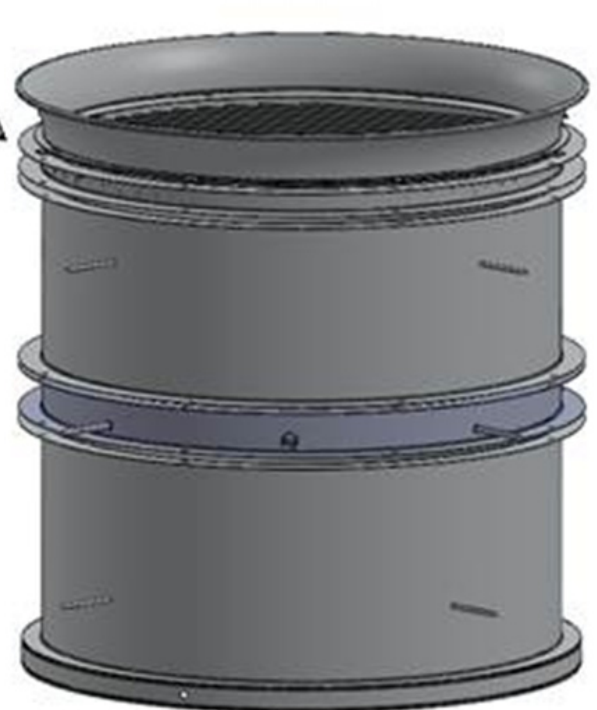

High Screen

Figure 4.8: Exploded views of flow barrel assembly for each test configuration. 


\subsubsection{Velocity Measurement Procedure}

The purpose of the velocity measurements is to quantify the turbulence that is entering the diffuser. The profile of the diffuser mouth is presented in Figure 4.9. The scales are defined so that $\mathrm{z} / \mathrm{W}=1$ and $\mathrm{y} / \mathrm{H}=1$ represent the edges of the opening to the mouth of the diffuser, although the traverses will span beyond those points. Note that $\mathrm{z} / \mathrm{W}=1$ represents half of the width of the mouth whereas $\mathrm{y} / \mathrm{H}=1$ represents the entire height of the mouth.

For each test configuration described in 4.2 .1 a set of velocity measurement traverses were performed. Two distinct traverse spans are included in the measurement set as shown in Figure 4.10.

One of the traverses spans longitudinally along the y-centerline and is referred to as the $0^{\circ}$ angle. The other traverse spans laterally offset at about $\mathrm{y} / \mathrm{H}=0.42$ from the $\mathrm{z}$-centerline so as to span across the middle of the mouth of the diffuser which is not in line with the center of the flow barrel. This traverse is referred to as the $90^{\circ}$ angle. The $0^{\circ}$ traverse reaches the bottom of the diffuser mouth opening and spans across the entire opening, whereas the $90^{\circ}$ traverse starts at the center and span across half of the opening.

A schematic of flow barrel with the probe access points is shown in Figure 4.11. This schematic shows the location of the probe access points at $d=0.75$ " and $d=15.7$ '. Note that the distance $d=15.7$ " refers to the extra measurement location that is used with the high screen configuration, although the schematic in Figure 4.11 exhibits the facility in the single screen configuration. 


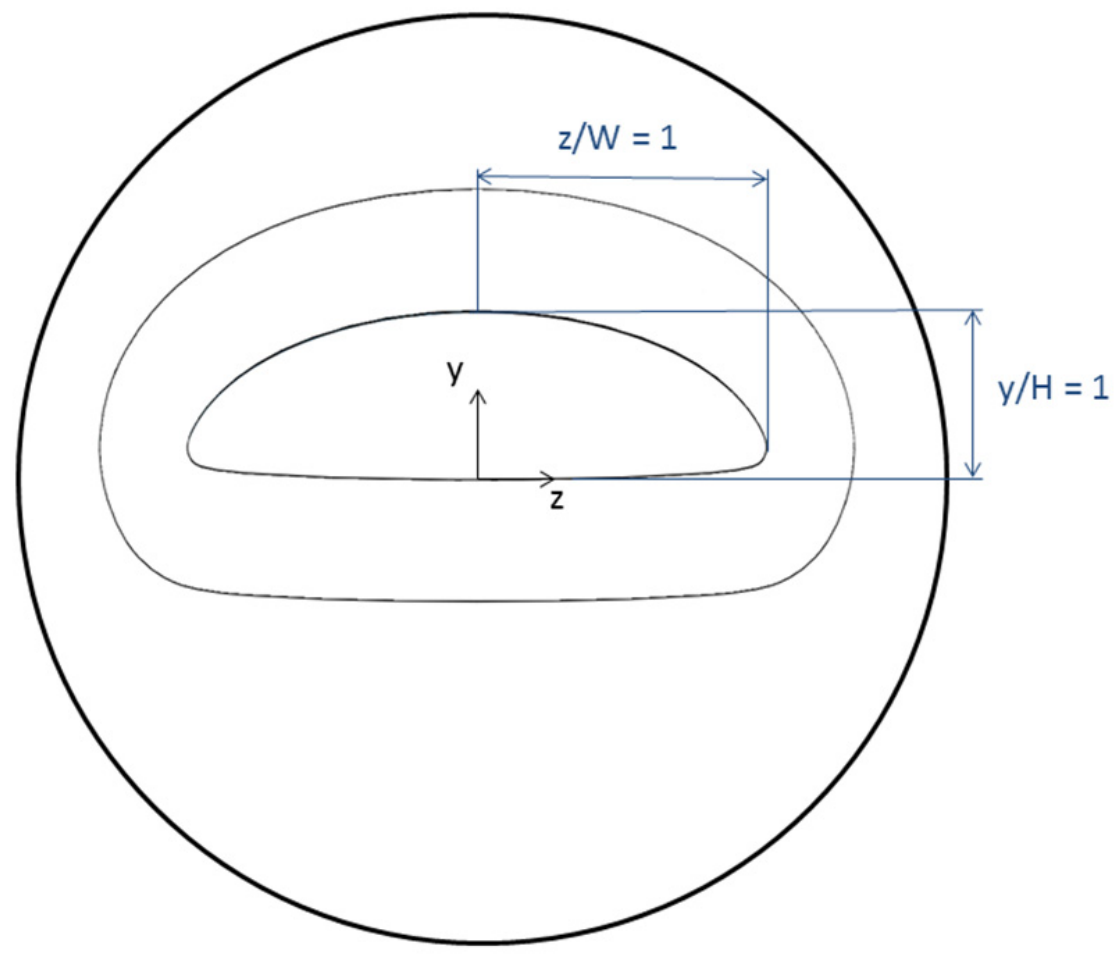

Figure 4.9: Scaled profile of the mouth of diffuser.

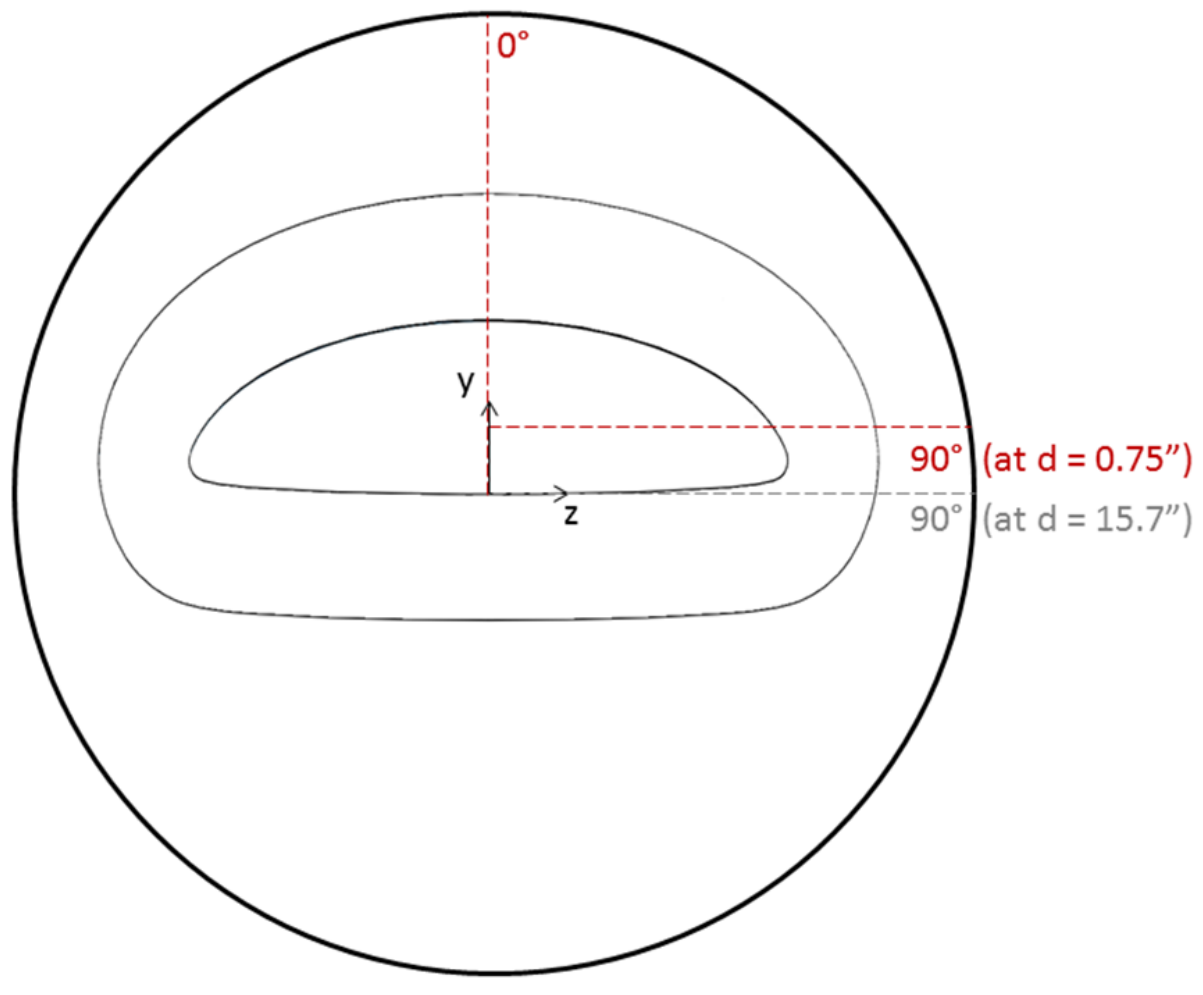

Figure 4.10: Velocity measurements traverse paths. 
In order to insert a hot film probe into the flow barrel to execute these traverses, holes were drilled into the side of the flow barrel and a pipe fitting was welded over the hole to provide support for the probe. These traverses were performed above the face of the diffuser mouth at $d$ $=0.75$ ". Additionally these traverses were performed above the face of the diffuser mouth at $\mathrm{d}=$ 15.7" in the fourth test configuration where the square bar grid was installed at a higher location $(d=37.3$ ") above the diffuser mouth. This was done to see the effects of the grid from roughly twenty inches downstream with minimal flow acceleration. The actual distance from the grid to the measurement location is $21.6 "$. The results from those measurements will be compared to the results of the single screen configuration where the measurements will be taken at 15.0" downstream of the grid with a high level of convective acceleration present. Note that the traverse at $90^{\circ}$ is offset at $\mathrm{d}=0.75^{\prime}$ to the line $\mathrm{y} / \mathrm{H}=0.42$ as opposed to the $90^{\circ}$ traverse at $\mathrm{d}=$ 15.7" which is along the z-centerline $\mathrm{y} / \mathrm{H}=0$.

Velocity measurements were taken at multiple positions along each traverse. In the case of the off-design Mach number study, the probe remained at $\theta=90^{\circ}$ and $\mathrm{z} / \mathrm{W}=0.41$.

The sampling frequency of the data acquisition software was set to $40 \mathrm{kHz}$ which was high enough for measuring the smallest (Komolgorov) turbulent length scales and well within the capabilities of the anemometry system. Each data point is taken for a span of one second to accurately measure the range of fluctuating velocity in the turbulent flow. A low-pass filter of $20 \mathrm{kHz}$ was programmed into the IFA-300 to remove noise from the signal without removing actual turbulent phenomena from the data. Probe movement along the traverses was executed manually with position repeatability of 0.05 ", or less than 0.007 for either $y / H$ or z/W. 


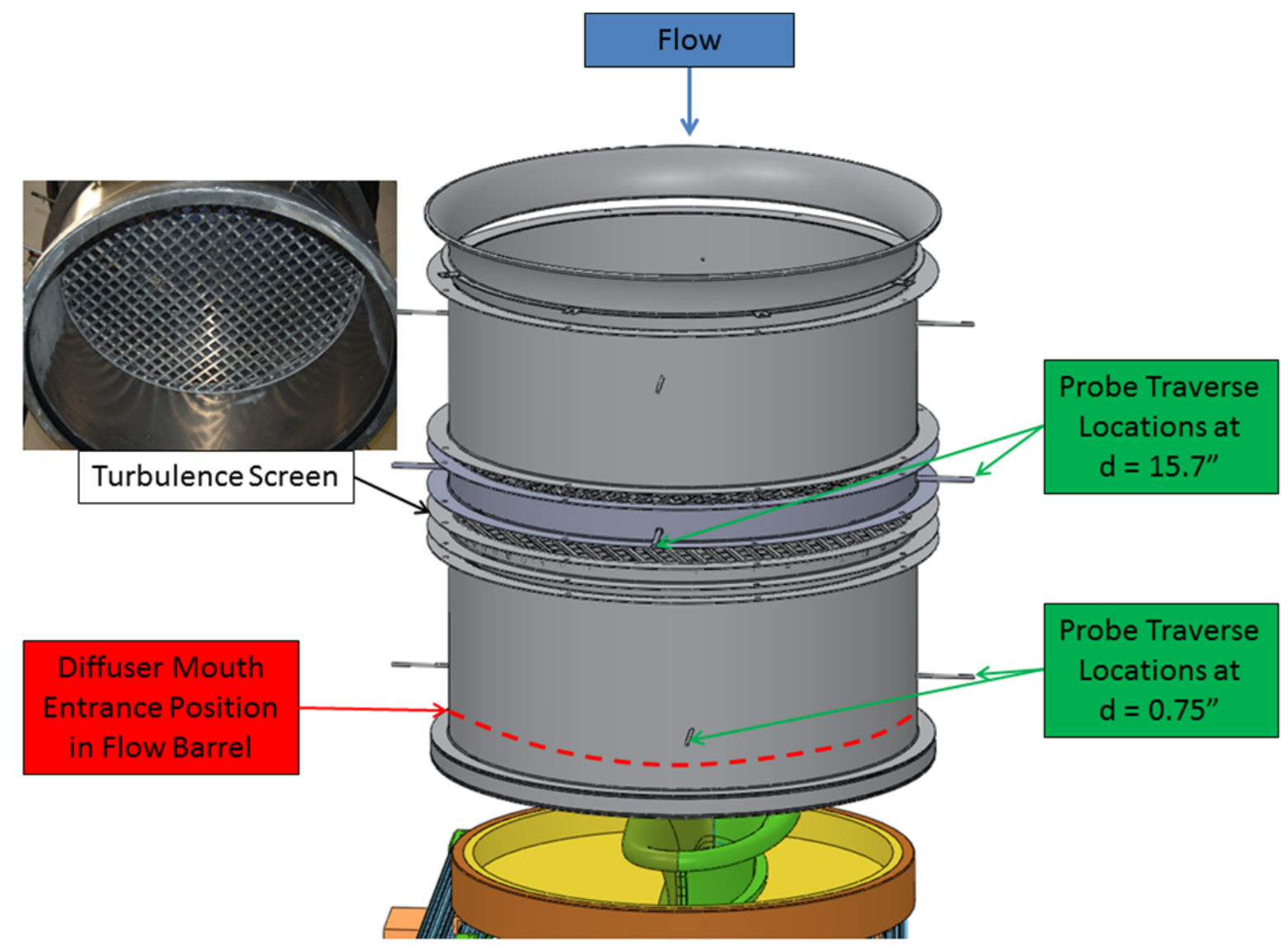

Figure 4.11: Flow barrel schematics (assembled in single screen configuration).

\subsection{Measurement Uncertainty}

There are a total of three types of measurements that are implemented in this study: inlet velocity measurements, axial static pressure measurements in the diffuser, and total pressure measurements at the AIP. Both the axial static pressure measurements and the total pressure measurements were made directly using the aforementioned Scanivalve DSA 3016 module. According to the user's manual, the Scanivalve DSA 3016 module has an accuracy of $\pm 0.05 \%$ of the full scale of the pressure range. The pressure range of the modules that were used in this study is $5 \mathrm{psi}$, which means the accuracy of its pressure readings is $\pm 0.0025 \mathrm{psi}$. 
The repeatability of the measurements was estimated through several preliminary facility tests. The repeatability, or precision, of the measurements is the uncertainty that is useful for comparing the results from each test configuration. For the axial static pressure measurements the repeatability was estimated to be within $1 \%$ of the measured valued, or $\pm 0.03 \mathrm{psi}$. The repeatability of the AIP total pressure measurements was estimated to be \pm 0.02 psi.

The velocity calibration process also depends on pressure measurements taken with the Scanivalve pressure transducer system. The velocity is calculated from pressure readings using Bernoulli's principle (see Appendix A for more detailed information.) With Bernoulli's principle, the velocity calculation is dependent on the pressure reading and atmospheric air density. The test facility where the calibrations took place was not fully air-conditioned and it is therefore estimated that the uncertainty of the air density due to temperature variations is \pm 0.003 $\mathrm{lb}_{\mathrm{m}} / \mathrm{ft}^{3}$. The uncertainty of the mean velocity due to calibration can be calculated from the following basic formula:

$$
u_{y}=\sqrt{\sum_{i}\left(u_{x_{i}} \frac{\partial y}{\partial x_{i}}\right)^{2}}
$$

where $u$ is the uncertainty, $y$ is the independent variable and $x_{i}$ is each dependent variable. With this formula the uncertainty in measured velocity depends on its value, resulting in a profile of the uncertainty across the range of expected values for this experiment. This profile is presented in Figure 4.12, with the uncertainty plotted as both a velocity value and a percentage. 


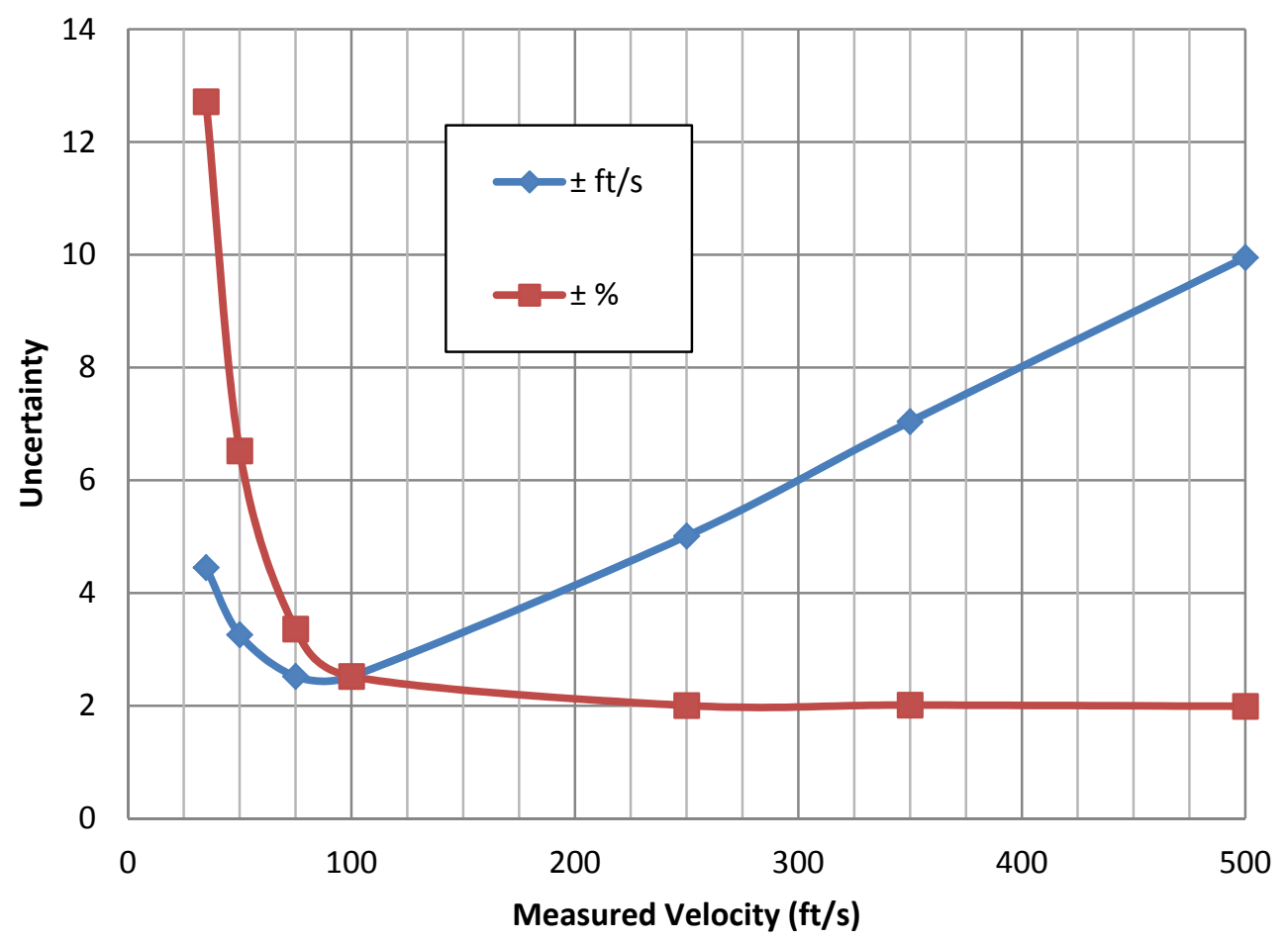

Figure 4.12: Measurement uncertainty of velocity data.

The uncertainty in units of velocity increases with increasing measured velocity after a measured velocity of $100 \mathrm{ft} / \mathrm{s}$, but the uncertainty as a percentage stays around $\pm 2 \%$ above measured velocity values of $100 \mathrm{ft} / \mathrm{s}$. The uncertainty as a percentages increases exponentially with decreasing velocity below measured values of $100 \mathrm{ft} / \mathrm{s}$, although the uncertainty in units of velocity does not surpass $\pm 5 \mathrm{ft} / \mathrm{s}$ for measured velocities below $100 \mathrm{ft} / \mathrm{s}$ within the expected range of velocities. 


\section{CHAPTER 5. RESULTS AND DISCUSSION}

The results presented in this chapter are divided and presented by each type of measurement that was taken. The data for each test configuration are presented in each section. In the first section the results of the velocity measurements taken to define and quantify the turbulence intensity entering into the serpentine diffuser are presented. Included in this section are calculations made to determine turbulence intensity. In the following sections, the axial static pressure measurements are presented followed by the total pressure measurements taken in the AIP which constitute the performance of the serpentine diffuser for each configuration.

\subsection{Inlet Velocity Measurements}

In this section the velocity data that were taken in the flow barrel upstream of the diffuser are presented. The mean velocity profiles (U) are presented first, followed by the standard deviation of the velocity data $\left(u_{r m s}\right)$. The turbulence intensity profiles, which are calculated from the standard deviation and mean velocity, are also presented. Finally, results from the additional high screen configuration study and off-design throat Mach number study are included in this section.

\subsubsection{Mean Velocity Profiles}

The mean velocity profiles for each of the test configurations are presented in Figure 5.1 and Figure 5.2. These two figures represent the velocity data that was taken with the hot film 
probe along each of the two traverse paths: $\theta=0^{\circ}$ and $90^{\circ}$. The diffuser throat Mach number was set at the design value of 0.691 .

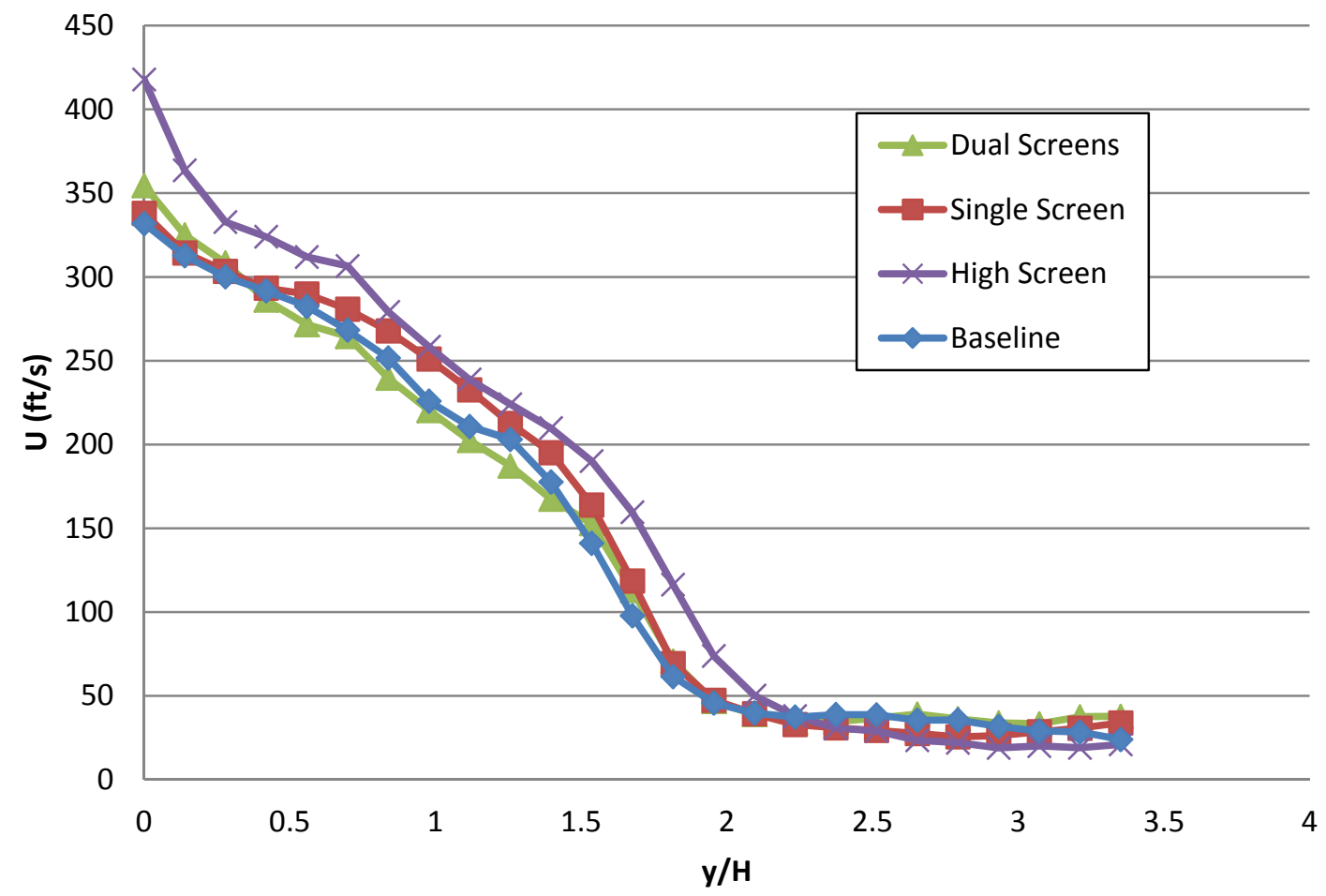

Figure 5.1: Mean velocity profiles at $\theta=0^{\circ}$ (along $\left.\mathrm{z} / \mathrm{W}=0\right), \mathrm{d}=0.75^{\prime}$ for each test configuration.

In each of the velocity profiles for the $\theta=0^{\circ}$ traverse shown in Figure 5.1, the mean streamwise velocity, $\mathrm{U}$, decreases from $\mathrm{y} / \mathrm{H}=0$ until about $\mathrm{y} / \mathrm{H}=2$ where each of the profiles levels off. This decrease resembles a parabolic channel flow profile between $\mathrm{y} / \mathrm{H}=0.5$ and $\mathrm{y} / \mathrm{H}$ $=2$. For $\mathrm{y} / \mathrm{H}<0.5$, the trends for each of the test configurations seem to increase in slope.

In Figure 5.2, the velocity profile trends for the $\theta=90^{\circ}$ traverse show a decreasing mean streamwise velocity almost across the entire traverse. The profiles for the dual screens and high screen configurations level off at $\mathrm{z} / \mathrm{W}=1.2$, the baseline at $\mathrm{z} / \mathrm{W}=1.3$ and the profile for the 
single screen configuration never levels off. Each of them decreases sharply between $\mathrm{z} / \mathrm{W}=1.1$ and $\mathrm{z} / \mathrm{W}=1.2$.

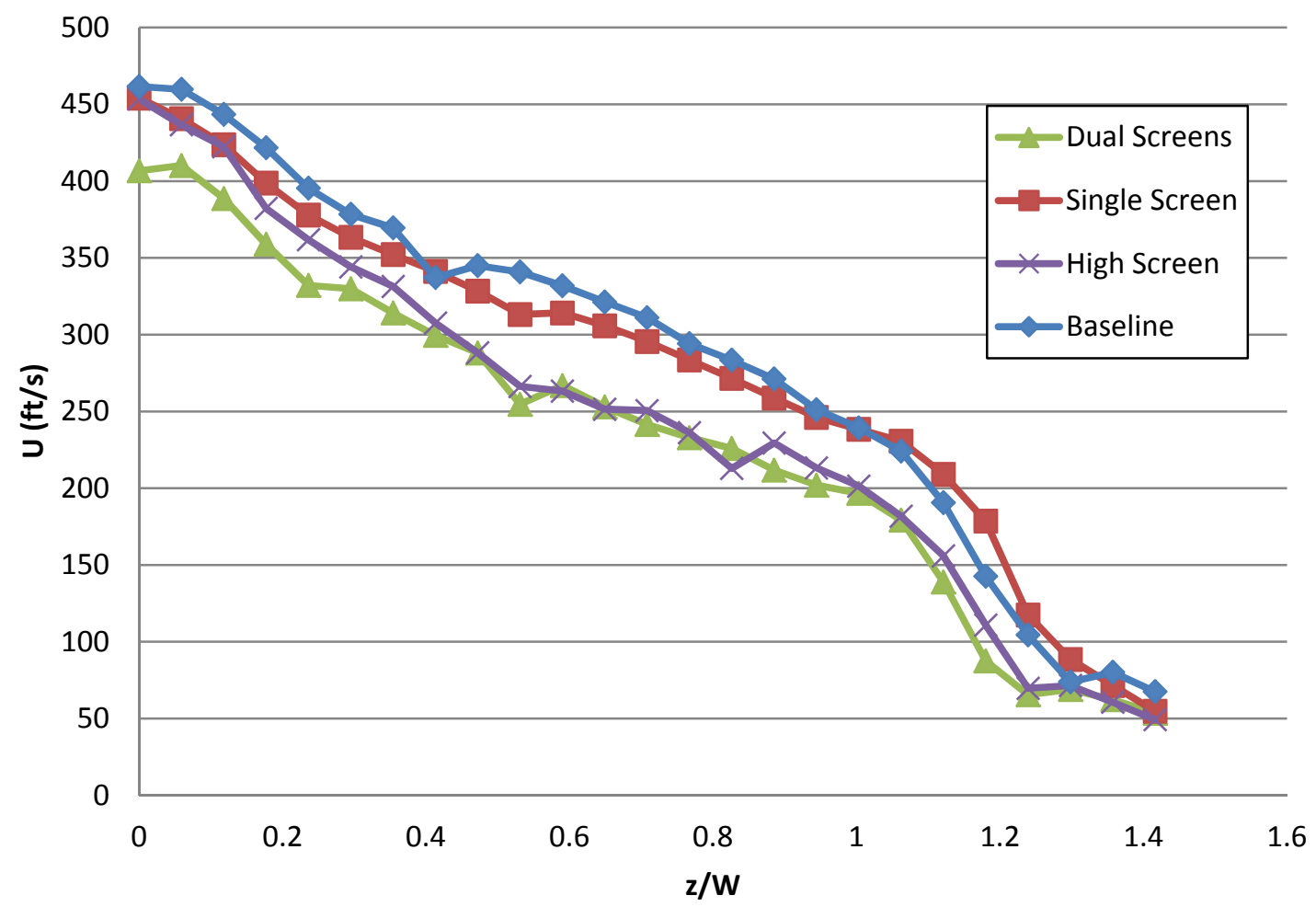

Figure 5.2: Mean velocity profiles at $\theta=90^{\circ}$ (along $\mathrm{y} / \mathrm{H}=0.42$ ), $\mathrm{d}=0.75^{\prime}$ for each test configuration.

The probe holder that is used to house the hot film probe is under a heavy load from the flow field when inserted to the center of the flow barrel. It is believed that this heavy load is causing the probe holder to droop, meaning the actual probe is closer to the mouth of the diffuser than $d=0.75$ ". The convective acceleration of the streamwise velocity field in this region is significantly high because the whole flow must accelerate to enter into the diffuser mouth which is a much smaller area than the flow barrel. Therefore, even a slight movement of the probe closer to the diffuser mouth would result in a significantly higher velocity reading. This effect can be observed in the mean velocity profiles. The effect is more pronounced towards the center 
where the load on the probe holder is greatest. At $\theta=0^{\circ}$, the velocity profile should actually peak at $y / H=0.42$ where the probe is directly above the center of the fish mouth, but because of the droop of the probe holder the measured velocity profile does not reflect that. This also explains why the velocities do not match where the two traverses meet at approximately $\mathrm{z} / \mathrm{W}=0$ and $\mathrm{y} / \mathrm{H}=0.42$ because the load on the probe holder is considerably less at $\theta=0^{\circ}$ as opposed to $\theta=90^{\circ}$ because the distance of the opening the probe spans is much less. This effect is consistent across the test configurations and therefore data points at each location can be accurately compared between configurations.

The purpose of presenting the velocity profiles in this study is to demonstrate that they are, or should be, close to the same magnitudes across the test configurations. Only the level of turbulence should be changing. There are however some anomalies that are not easily explained. It is unclear why the velocity is approximately $75 \mathrm{ft} / \mathrm{s}$ higher at $\theta=0^{\circ}$, and $\mathrm{y} / \mathrm{H}=0$ for the high screen configuration. It is also unclear why the entire velocity profile is lower at $\theta=90^{\circ}$ for both the high screen and the dual screens configurations. The fact that this behavior does not repeat for both of the traverse positions $\left(\theta=0^{\circ}\right.$ and $\left.\theta=90^{\circ}\right)$ does not support that a measurement bias was present when measuring those two profiles (high screen and dual screen at $\theta=90^{\circ}$ ). These unexplained anomalies could indicate that there is unexpected variation in the overall flow rate from case to case. In general though, the profiles do match up well in both magnitude and trend. The averages of the $\theta=0^{\circ}$ profiles vary less than $15 \%$ and the averages of the $\theta=90^{\circ}$ profiles vary less than $22 \%$. 


\subsubsection{Fluctuating Velocity and Turbulence Intensity Profiles}

The $u_{r m s}$ at a given point in the flow field is an indication of the level of turbulence of the flow at that point. It can be seen in the $u_{r m s}$ profiles that the turbulence screens do have the effect of increasing the level of turbulence of the flow. The standard deviation is calculated by taking the root-mean-square of the fluctuating velocity, $u$ ':

$$
u_{r m s}=\sqrt{\overline{\left(u^{\prime}\right)^{2}}}
$$

The standard deviation of the velocity profiles $\left(u_{r m s}\right)$ are presented in Figure 5.3 and Figure 5.4 for $\theta=0^{\circ}$ and $90^{\circ}$, respectively. The standard deviation was calculated using the 40,000 velocity readings that were taken over a period of one second at each location along the traverses.

The $u_{r m s}$ profiles of the $\theta=0^{\circ}$ traverse shown in Figure 5.3 show some variation across the diffuser mouth, from $y / H=0$ to $y / H=1$. All of the profiles, except that of the high screen configuration, see a decrease in $u_{r m s}$ from $\mathrm{y} / \mathrm{H}=0$ to $\mathrm{y} / \mathrm{H}=0.25$. Near $\mathrm{y} / \mathrm{H}=1.8$ there is a spike that is seen in all of the profiles (except the profile of the high screen configuration whose spike peaks at about $\mathrm{y} / \mathrm{H}=1.9)$. The spike at $\mathrm{y} / \mathrm{H}=1.9$ for the high screen configuration is also nearly double in magnitude compared to the profiles of the single screen and dual screens configurations despite having a lower $u_{r m s}$ across the diffuser mouth. These spikes are likely due to the flow circulation that exists in the corner of the flow barrel past the lip of the diffuser. The circulation in this region is highly unsteady and unpredictable. The circulating flow in the bottom corner of the flow barrel is indeed turbulent flow, but it is not freestream turbulence that is passing into the diffuser and is therefore not of interest to this study. 


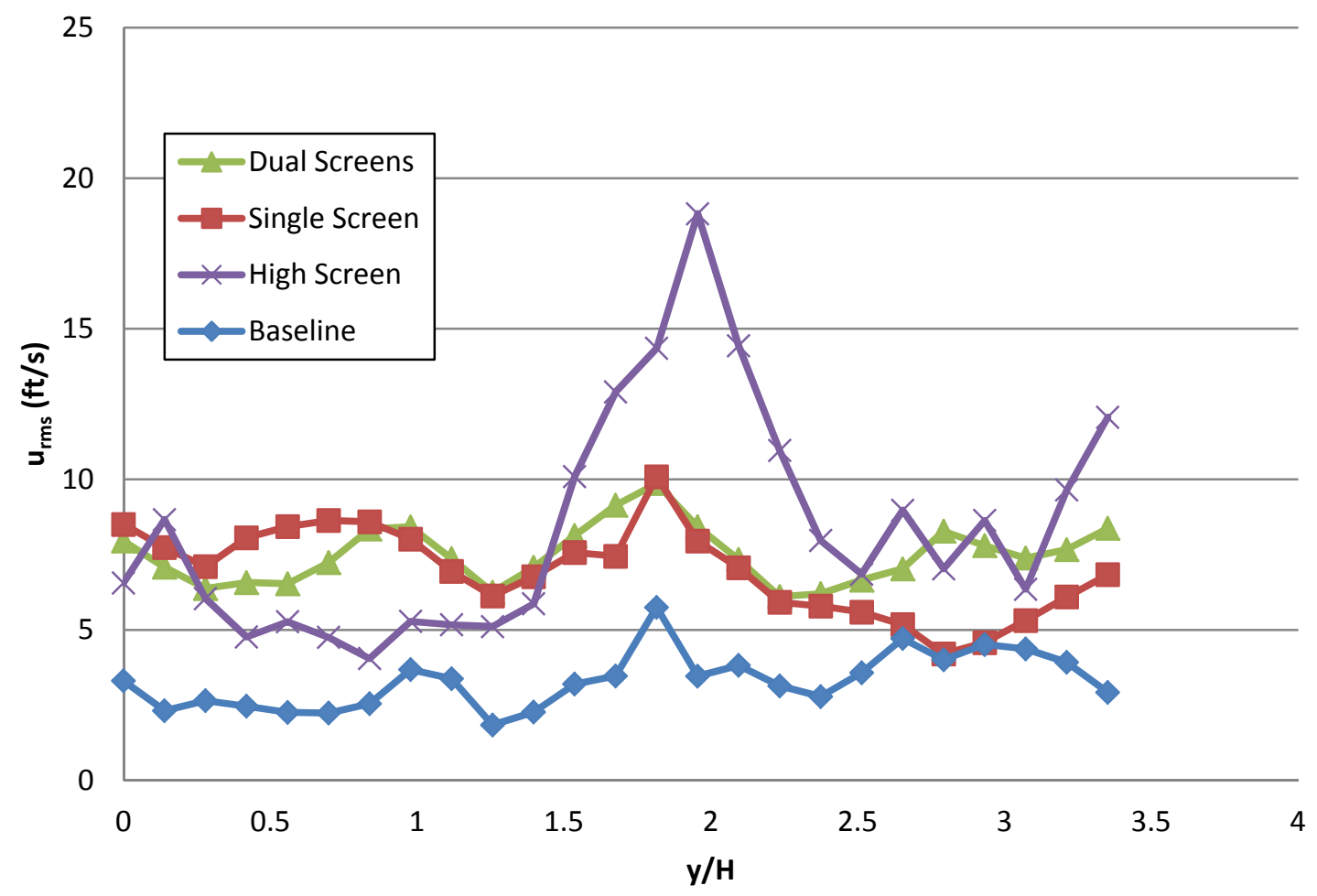

Figure 5.3: $u_{r m s}$ profiles at $\theta=0^{\circ}($ along $\mathrm{z} / \mathrm{W}=0), \mathrm{d}=0.75$ " for each test configuration.

Upon observing the difference between the baseline configuration and the single screen configuration, a clear increase in $u_{r m s}$ is apparent between the profiles. The average $u_{r m s}$ across the face of the diffuser mouth in the $\theta=0^{\circ}$ traverse is $193 \%$ higher for the single screen configuration than for the baseline. It is $108 \%$ higher for the high screen configuration and $170 \%$ higher for the dual screens configuration. For this traverse, the single screen configuration produced the highest level of $u_{r m s}$. The dual screens configuration does not demonstrate an increase in $u_{r m s}$ over the single screen configuration. Although it was hypothesized that this configuration would increase the level of turbulence above that of the single screen configuration, it was still uncertain what a dual screen configuration would produce due to the lack of previous data. 
Each of the $u_{r m s}$ profiles of the $\theta=90^{\circ}$ traverse shown in Figure 5.4 are increasing from $\mathrm{z} / \mathrm{W}=0$ to about $\mathrm{z} / \mathrm{W}=0.15$, with the exception of the dual screens configuration whose profile is relatively flat across the face of the diffuser mouth. The $u_{r m s}$ of the baseline configuration is 0 at $\mathrm{z} / \mathrm{W}=0$. Each of the profiles that initially saw an increase eventually see a decrease as well beginning between $\mathrm{z} / \mathrm{W}=0.15$ and $\mathrm{z} / \mathrm{W}=0.4$. Again, the flow circulation region is apparent in the data where each of the profiles exhibits a spike for $\mathrm{z} / \mathrm{W}>1$. The appearance of the circulation right at the lip of the diffuser mouth $(\mathrm{z} / \mathrm{W}=1)$ is likely due to this edge of the lip's much closer proximity to the wall of the flow barrel (see Figure 4.9). In the $\theta=90^{\circ}$ traverse it appears to become very significant just beyond the lip of the diffuser. Therefore the data of interest are the data points from the center up to the lip of the diffuser for the $\theta=90^{\circ}$ traverse.

The $u_{r m s}$ profile of the baseline configuration is not flat across diffuser mouth opening. This suggests that there may have been some installation effects on the turbulence intensity of the flow other than the turbulence screens. The average $u_{r m s}$ across face of the diffuser mouth in the $\theta=90^{\circ}$ traverse was $113 \%$ higher for the single screen configuration than for the baseline. It was $58 \%$ higher for the high screen configuration and $151 \%$ higher for the dual screens configuration. For the $\theta=90^{\circ}$ traverse, the dual screens configuration showed a significant increase in $u_{r m s}$ when compared to the single screen configuration. For both of the traverses, the high screen configuration exhibited an increase in $u_{r m s}$ from the baseline configuration, but to a smaller degree than either the single screen or baseline configurations. 


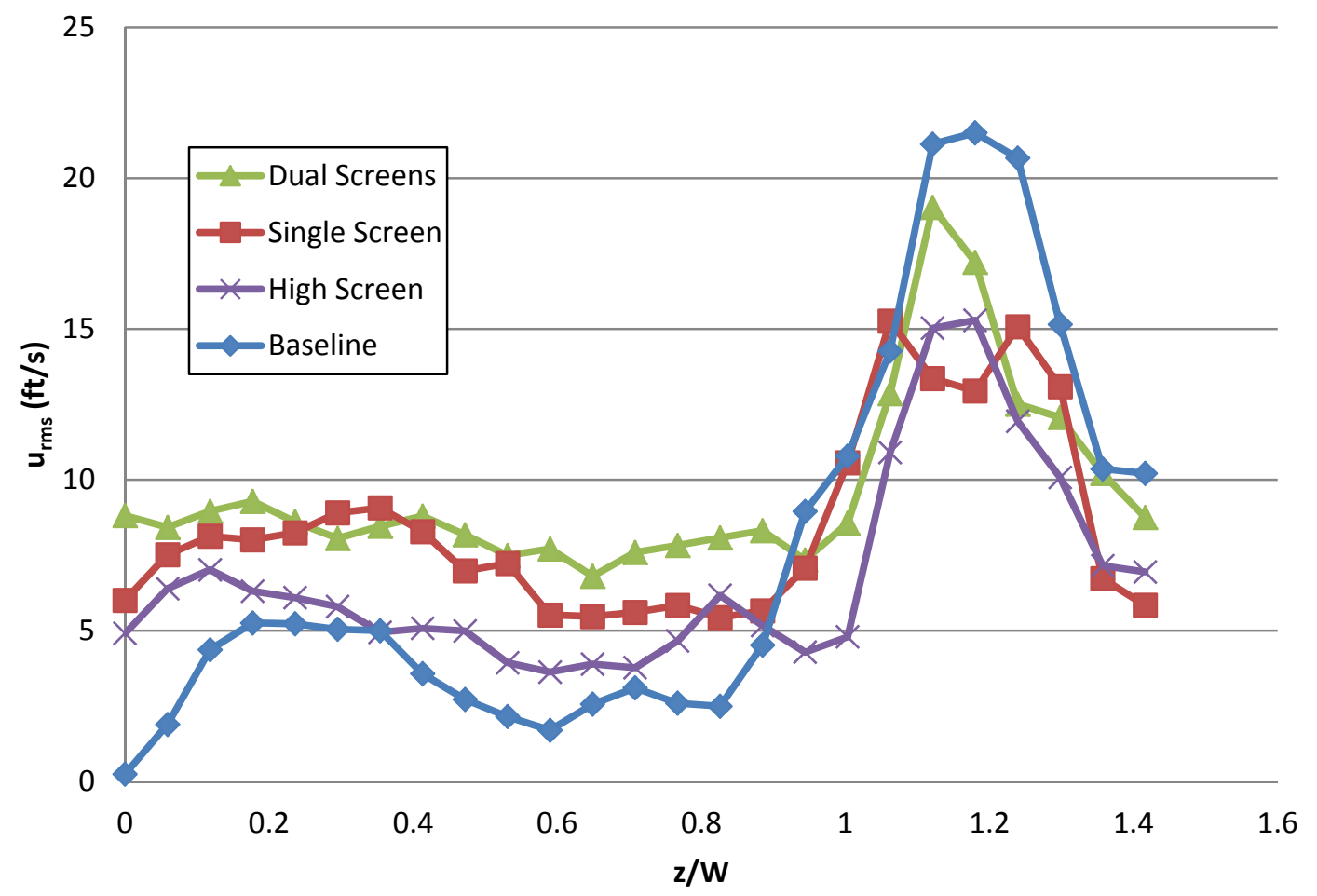

Figure 5.4: $u_{r m s}$ profiles at $\theta=90^{\circ}($ along $\mathrm{y} / \mathrm{H}=0.42), \mathrm{d}=0.75$ " for each test configuration.

As previously mentioned, the diffuser mouth opening is much smaller than the crosssectional area of the flow barrel. Hence a flow circulation region is present outside of the diffuser mouth at the end of the flow barrel in between the walls of the flow barrel and walls of the diffuser. An example of this is shown in Figure 5.5 which is a CFD generated 3-D velocity contour of the flow entering the model diffuser from the flow barrel (also by Dr. Darius Sanders). Figure 5.5 demonstrates the flow behavior at the end of the flow barrel outside of the diffuser mouth. The wall-bounded flow is highly unsteady, turbulent and circulating. Although some of this circulating flow does make its way into the diffuser, the CFD analysis also demonstrated that the streamlines going straight into the diffuser are not affected by it. This high turbulence intensity region is apparent in each of the $u_{r m s}$ profiles (for $\mathrm{y} / \mathrm{H}>1.5$ and $\mathrm{z} / \mathrm{W}>1$ ). As previously noted, this region is of little interest to this study. In order to better compare the 
turbulence intensity of each flow entering the diffuser, the axes for the plots of the turbulence intensity have been re-scaled and are presented in Figure 5.6 and Figure 5.7.

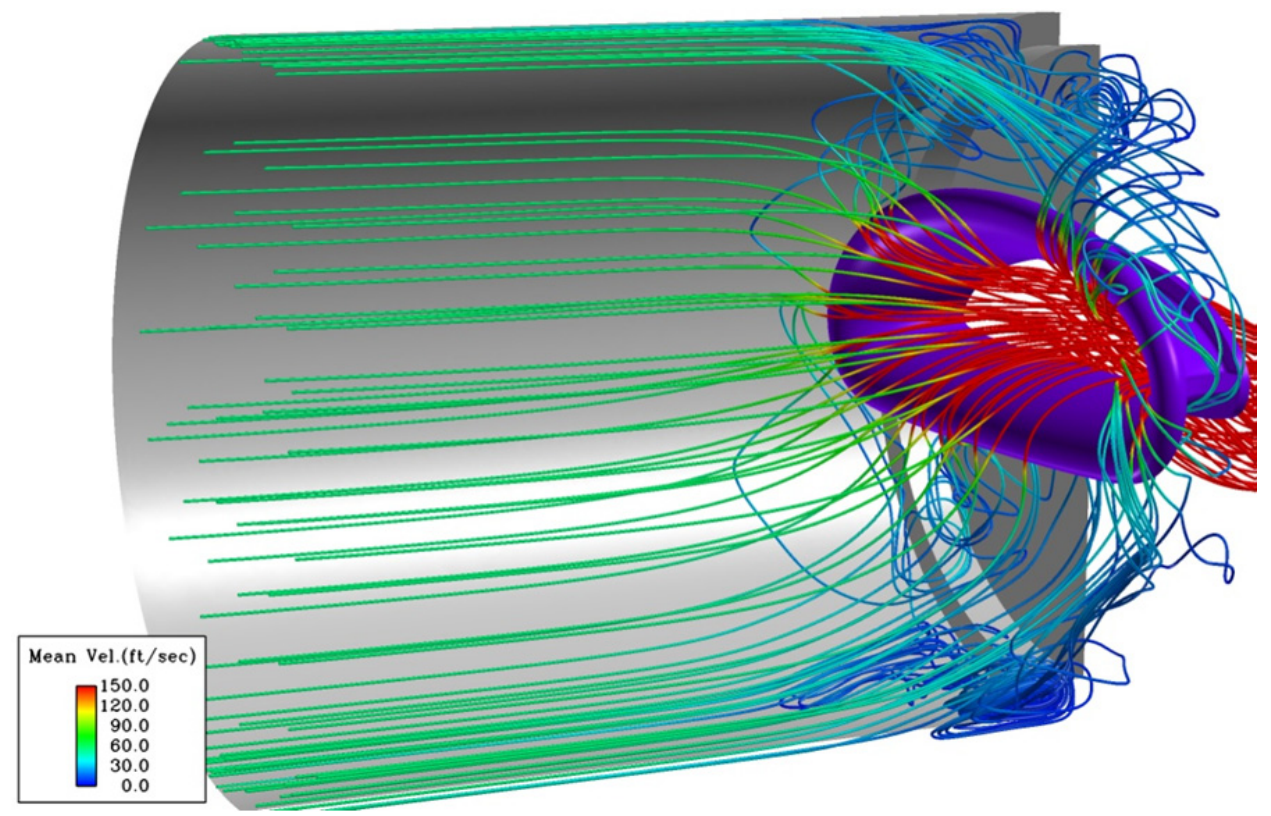

Figure 5.5: CFD velocity lines of flow entering model diffuser from flow barrel.

The turbulence intensity quantity provides additional insight to into the characteristics of the turbulent flow. By normalizing by the time-averaged local bulk velocity, the $T u$ quantity appropriately puts the level of turbulence into perspective relative to the flow field and helps eliminate velocity biases in measurement.

The $T u$ profile of the single screen configuration for the $\theta=0^{\circ}$ traverse as shown in Figure 5.6 is relatively uniform around $1 \%$ from $y / H=0$ to about $y / H=0.8$. Between $y / H=0.8$ and $\mathrm{y} / \mathrm{H}=1.25$ there is a hump that reaches $1.5 \%$ turbulence intensity but decreases back to about $1 \%$. The profile of the high screen configuration initially sees a spike from $1.5 \%$ at $\mathrm{y} / \mathrm{H}=$ 0 to $2.5 \%$ at $y / H=0.2$. By $y / H=0.45$ its value has decreased to $1.5 \%$ where it remains fairly steady until $\mathrm{y} / \mathrm{H}=0.85$ where it begins to increase again reaching $2.3 \%$ at $\mathrm{y} / \mathrm{H}=1.25$ before being affected by the circulating flow for $\mathrm{y} / \mathrm{H}>1.3$. The profile of the single screen 
configuration is $2.5 \%$ at $\mathrm{y} / \mathrm{H}=0$ and after a slight decrease begins to gradually increase at $\mathrm{y} / \mathrm{H}=$ 0.3 and reaches as high as $3.2 \%$ turbulence intensity at $\mathrm{y} / \mathrm{H}=0.85$. Likewise, the $T u$ profile of the dual screens configuration sees a slight decrease from $2.2 \%$ at $y / H=0$ until $y / H=0.3$ where it begins to gradually increase. At $\mathrm{y} / \mathrm{H}=0.7$ a more drastic increase is observed until about $\mathrm{y} / \mathrm{H}$ $=1$ where the turbulence intensity reaches $3.8 \%$.

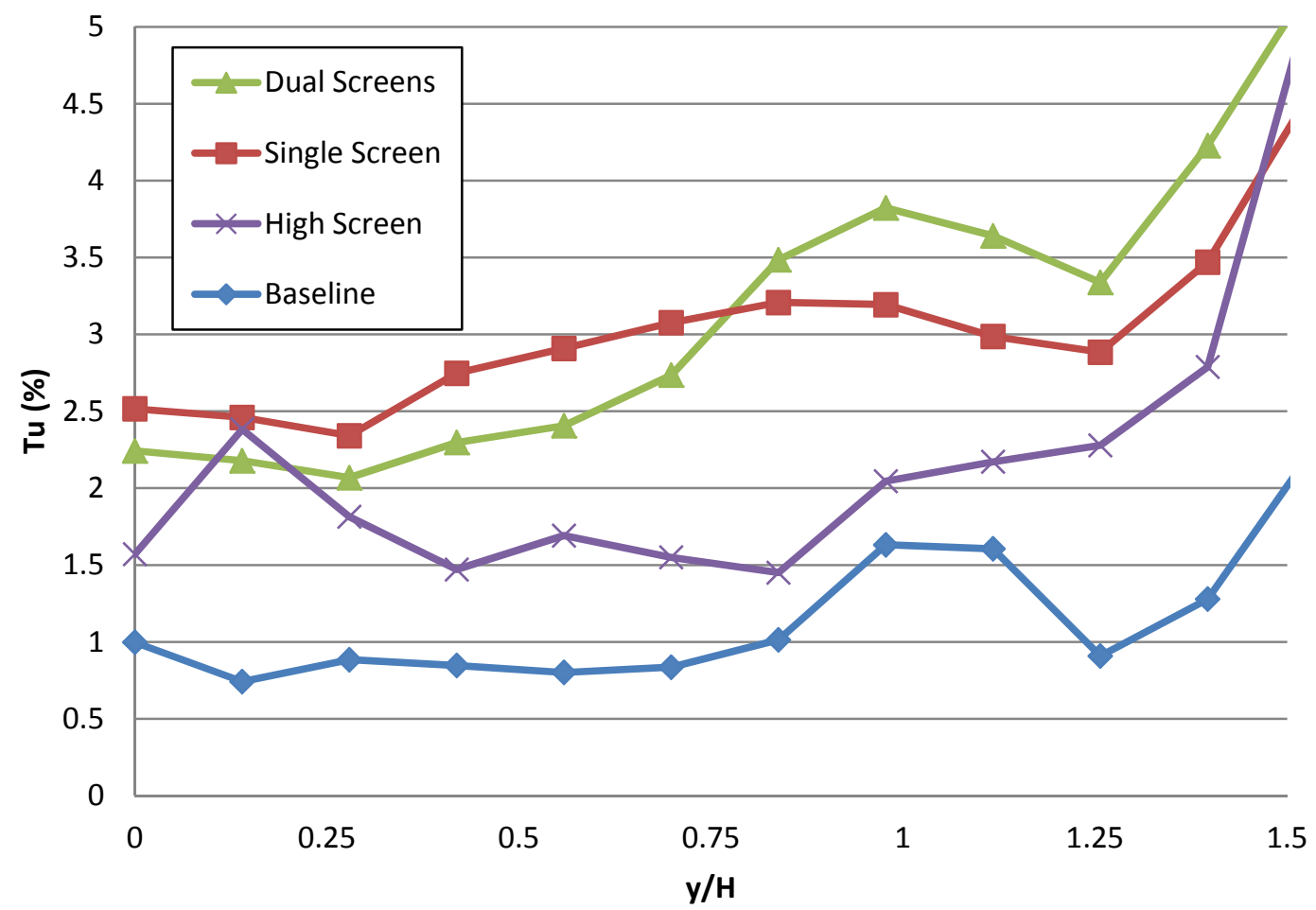

Figure 5.6: $T u$ profiles at $\theta=0^{\circ}$ (along $\mathrm{z} / \mathrm{W}=0$ ), $\mathrm{d}=0.75^{\prime}$ " for each test configuration.

A clear separation in turbulence intensity is visible between the four different test configurations for the $\theta=0^{\circ}$ traverse profiles. The average turbulence intensity across the profile for the high screen represents an $80 \%$ increase from that of the baseline configuration, whereas the profile of the single screen configuration represents a $176 \%$ increase. Interestingly, the average turbulence intensity of the dual screens configuration represents a $175 \%$ increase 
which is virtually the same as the increase of the single screen configuration. This is a departure from the comparisons of the $\theta=0^{\circ}$ traverse $u_{r m s}$ profiles where the single screen configuration produced a clear lead over the dual screens profile. The flow with the dual screen configuration is substantially higher in turbulence intensity in the region right at the upper surface of the diffuser mouth opening. This observation is significant as the separation region inside of the diffuser, which is an area of interest in this study regarding diffuser performance, lies along the upper surface.

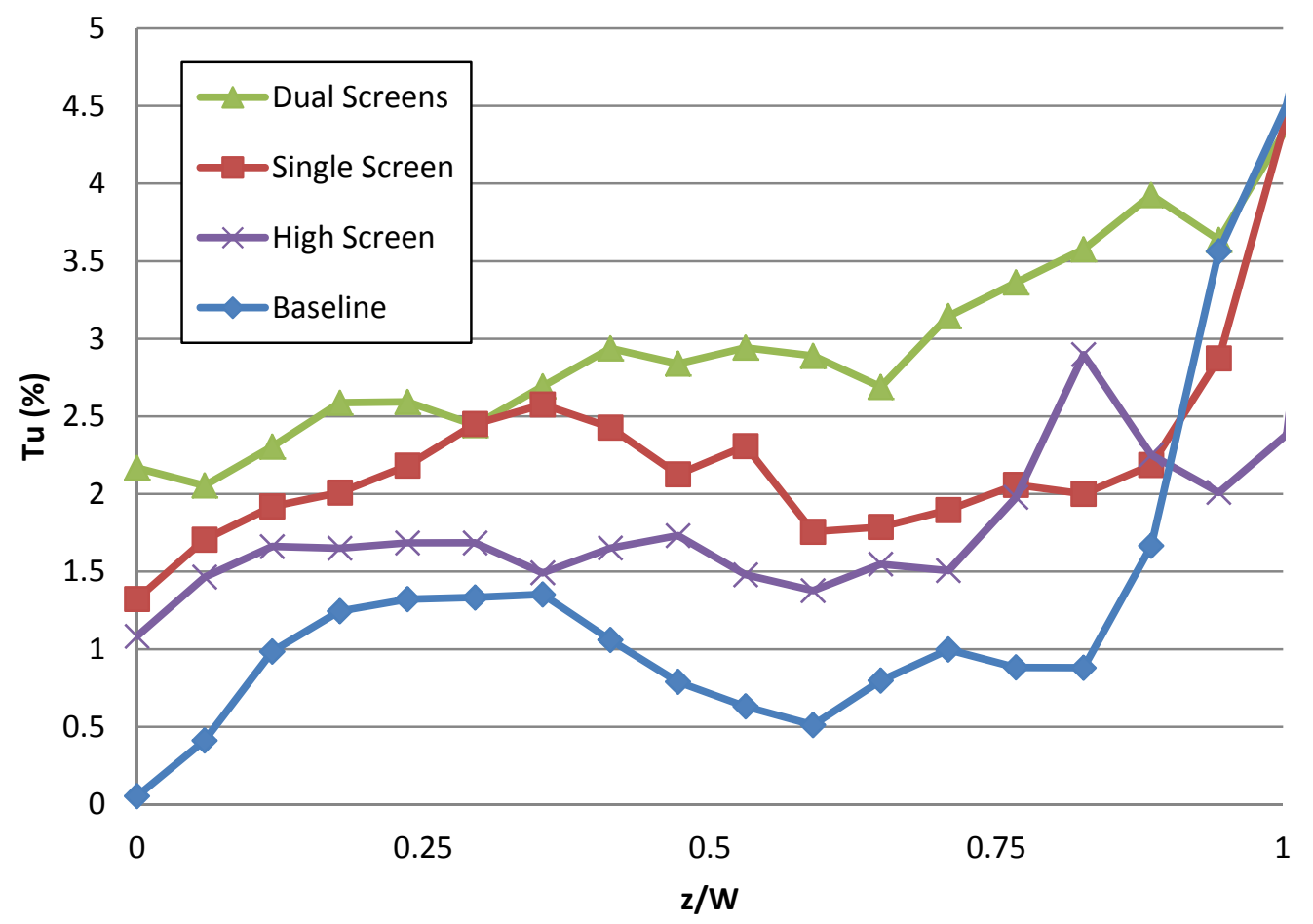

Figure 5.7: $T u$ profiles at $\theta=90^{\circ}$ (along $\left.\mathrm{y} / \mathrm{H}=0.42\right), \mathrm{d}=0.75$ ” for each test configuration.

The $T u$ profile of the single screen configuration for the $\theta=90^{\circ}$ traverse as shown in Figure 5.7 begins at $0 \%$ at $\mathrm{z} / \mathrm{W}=0$ and increases to about $1.4 \%$ at $\mathrm{z} / \mathrm{W}=0.2$ where it plateaus until $\mathrm{z} / \mathrm{W}=0.35$. It then decreases down to $0.5 \%$ at about $\mathrm{z} / \mathrm{W}=0.6$ then increases up to $1 \%$ at $\mathrm{z} / \mathrm{W}=0.7$ where it remains relatively flat until affected by circulating flow beyond $\mathrm{z} / \mathrm{W}=0.85$. 
The profile of the high screen configuration begins at about $1 \%$ at $\mathrm{z} / \mathrm{W}=0$ and increases to about $1.6 \%$ at $\mathrm{z} / \mathrm{W}=0.15$. It hovers between $1.5 \%$ and $1.6 \%$ turbulence intensity until $\mathrm{z} / \mathrm{W}=0.7$ where it begins to spike up to $2.9 \%$ at $\mathrm{z} / \mathrm{W}=0.85$ then decreases down to $2 \%$ just before the lip of the diffuser. The profile of the single screen configuration begins at $1.4 \%$ at $\mathrm{z} / \mathrm{W}=0$ then gradually increases up to $2.6 \%$ turbulence intensity at $\mathrm{z} / \mathrm{W}=0.35$. It then erratically decreases to $1.8 \%$ at $\mathrm{z} / \mathrm{W}=0.6$ then begins a slow increase to $2 \%$ by $\mathrm{z} / \mathrm{W}=0.75$ before being affected by circulating flow beyond $\mathrm{z} / \mathrm{W}=0.9$. The profile of the dual screen configuration begins at $2.1 \%$ at $\mathrm{z} / \mathrm{W}=0$ and erratically increases to $2.9 \%$ at $\mathrm{z} / \mathrm{W}=0.4$. It then hovers between $2.6 \%$ and $2.9 \%$ until $\mathrm{z} / \mathrm{W}=0.65$ where it begins to sharply increase up to $3.9 \%$ turbulence intensity at $\mathrm{z} / \mathrm{W}=$ 0.85 .

Again a clear separation in turbulence intensity is visible between the four different test configurations for the $\theta=90^{\circ}$ traverse profiles. The average turbulence intensity across the profile for the high screen configuration represents an $82 \%$ increase from that of the baseline configuration, whereas the profile of the single screen configuration represents a $119 \%$ increase. The average turbulence intensity of the dual screens configuration represents a $203 \%$ increase from the baseline. The turbulence intensity of the flow in the dual screen configuration maintains a lead over that of the single screen configuration across the profile. In both traverses, the turbulence intensity of the flow in the high screen configuration mostly falls in between that of the baseline and the single screen configurations.

Again it is noted that the turbulence intensity profiles of the flow in the single screen and baseline configurations follow similar trends. This is most notable in the $\theta=90^{\circ}$ profile. The profiles of the flow in the other two configurations, while demonstrating an expected magnitude of turbulence intensity, have less similar trends. 


\subsubsection{Turbulence Generation without Flow Acceleration}

In this section, the velocity and turbulence intensity profiles at a distance of 21.6 " downstream of the square bar grid configured in the high screen configuration will be presented and discussed (see Table 4.1 and Figure 4.11). As discussed in Chapter 4, this study was performed to observe the difference in dissipation of turbulence generated by the square bar grid in the absence of the flow acceleration that is taking place relatively near the entrance to the mouth of the diffuser. According to the correlation of Roach [16], the turbulence intensity of the flow twenty inches downstream of the square bar turbulence grid, as used in the present study, should be approximately $8 \%$.

The velocity profiles of the high screen configuration at $d=15.7$ " compared to the previously shown single screen configuration profiles at $d=0.75$ " are presented in Figure 5.8 and Figure 5.9 for $\theta=0^{\circ}$ and $90^{\circ}$, respectively. The single screen profiles measured at $d=0.75$ ” are at a distance of 15.0" from the square bar turbulence screen and the high screen profiles measured at $\mathrm{d}=15.7$ " are at a distance of 21.6 " from the same square bar turbulence screen.

In the $\theta=0^{\circ}$ traverse in Figure 5.8, the high screen velocity profile starts at $85 \mathrm{ft} / \mathrm{s}$ at $\mathrm{y} / \mathrm{H}$ $=0$ and gradually decreases to slightly below $50 \mathrm{ft} / \mathrm{s}$ at $\mathrm{y} / \mathrm{H}=3.4$. This is in contrast to the single screen profile which starts at $340 \mathrm{ft} / \mathrm{s}$ at $\mathrm{y} / \mathrm{H}=0$ and decreases to $50 \mathrm{ft} / \mathrm{s}$ at $\mathrm{y} / \mathrm{H}=2$. It then hovers between $30 \mathrm{ft} / \mathrm{s}$ and $40 \mathrm{ft} / \mathrm{s}$ from $\mathrm{y} / \mathrm{H}=2$ until $\mathrm{y} / \mathrm{H}=3.4$. 


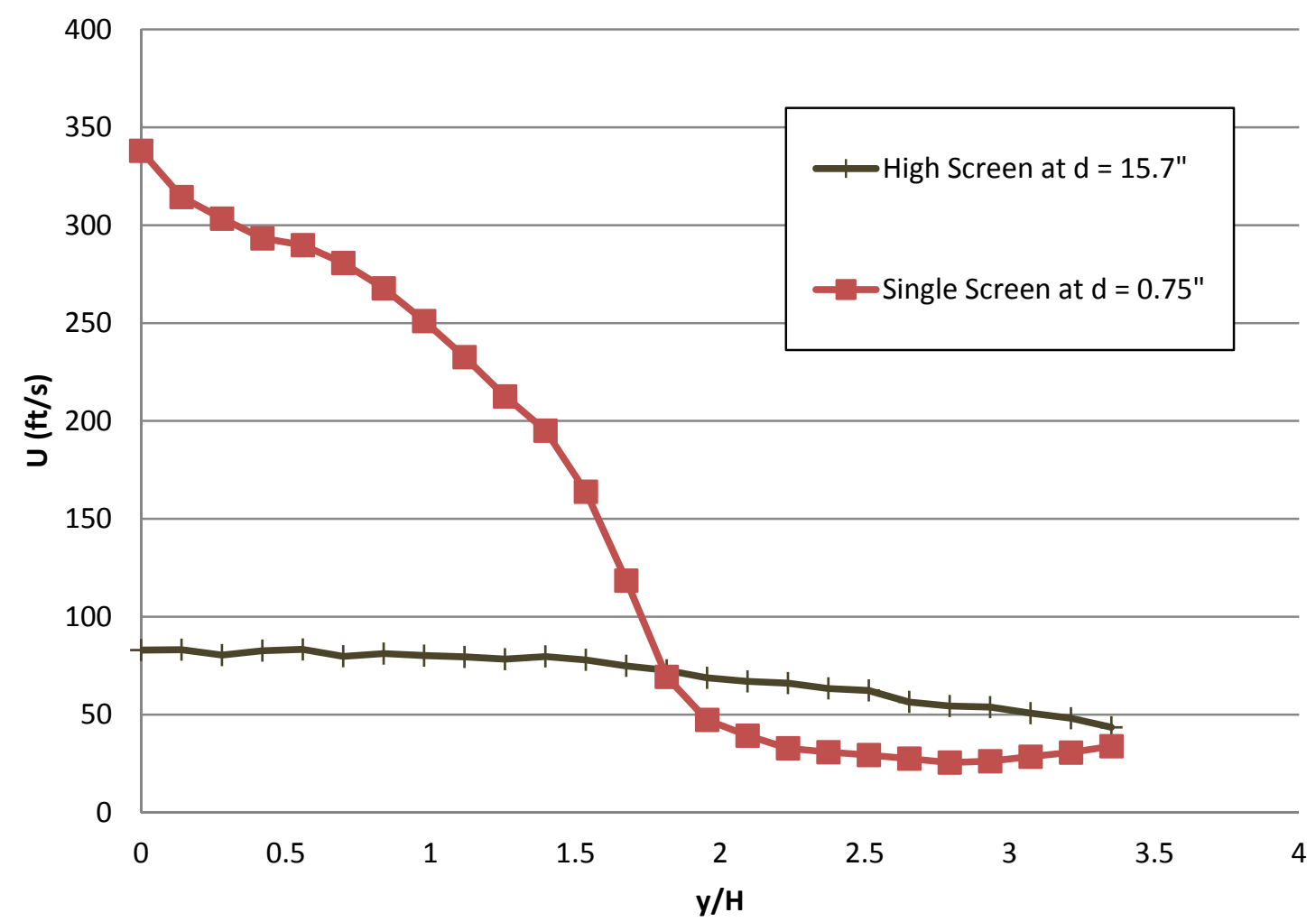

Figure 5.8: Mean velocity profiles at $\theta=0^{\circ}($ along $\mathrm{z} / \mathrm{W}=0)$, for the high screen configuration.

In the $\theta=90^{\circ}$ traverse in Figure 5.9, the velocity profiles are similar to that of the $\theta=0^{\circ}$ traverse profiles. The high screen velocity profile decreases from $80 \mathrm{ft} / \mathrm{s}$ at $\mathrm{z} / \mathrm{W}=0$ to $50 \mathrm{ft} / \mathrm{s}$ at $\mathrm{z} / \mathrm{W}=1.4$. The single screen velocity profile decreases from $450 \mathrm{ft} / \mathrm{s}$ at $\mathrm{z} / \mathrm{W}=0$ to $50 \mathrm{ft} / \mathrm{s}$ at $\mathrm{z} / \mathrm{W}$ $=1.4$.

The measurement height of $d=15.7$ " for the high screen in this particular investigation is the same as the location height of the turbulence screen for the single screen test configuration. Hence the difference between the high screen and single screen velocity profiles in Figure 5.8 and Figure 5.9 represent the change in velocity from the turbulence screen to the measurement location of the single screen test configuration. 


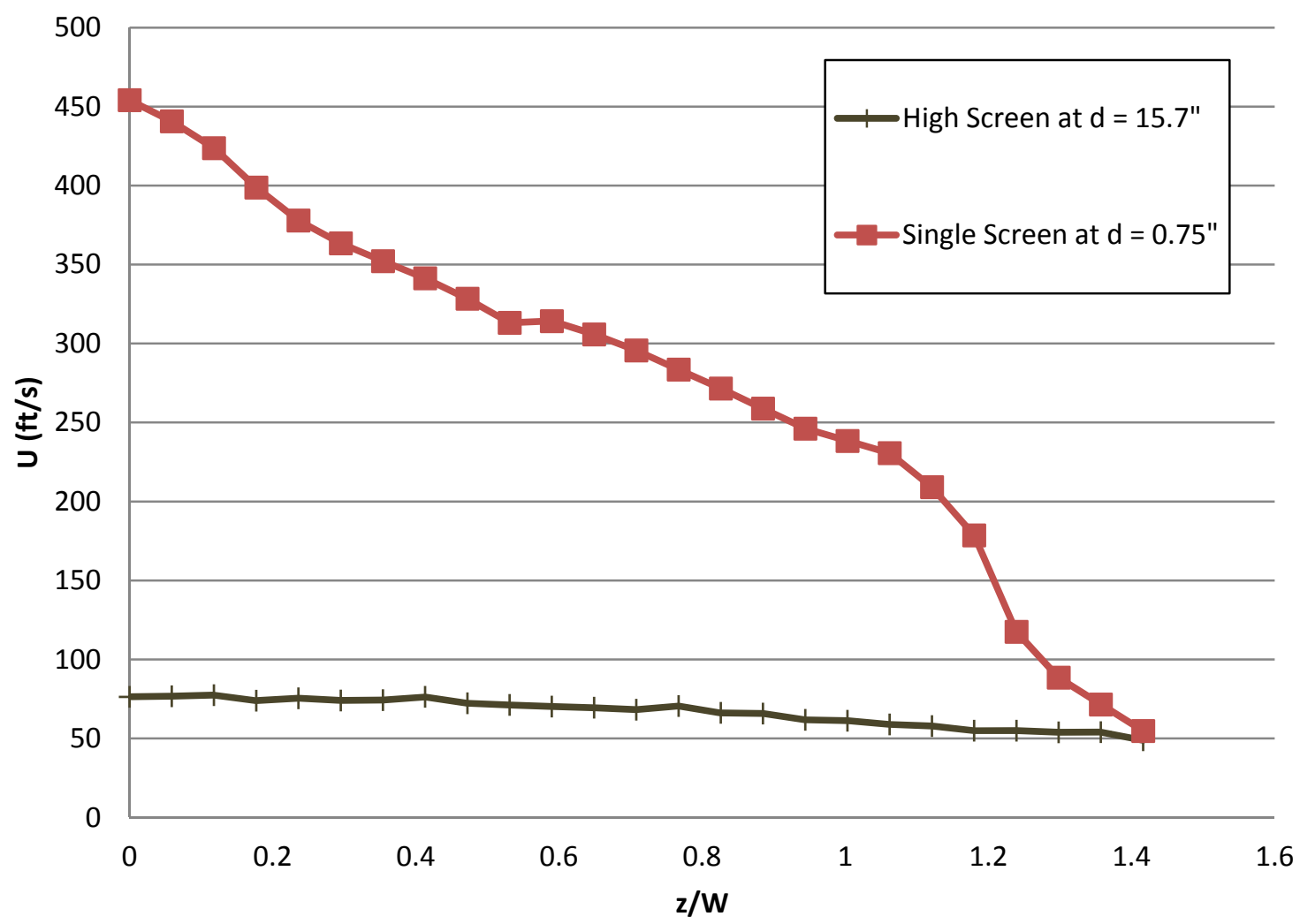

Figure 5.9: Mean velocity profiles at $\theta=90^{\circ}$ for the high screen configuration.

It is important to note that the two traverses at $\theta=90^{\circ}$ were each performed along a different value of $y / H$. The traverse at $d=0.75$ " for the single screen configuration was performed along $\mathrm{y} / \mathrm{H}=0.42$ (as previously explained), whereas the traverse at $\mathrm{d}=15.7$ " for the high screen configuration was performed along the flow barrel centerline $(\mathrm{y} / \mathrm{H}=0)$.

Both velocity profiles at $d=15.7$ " are flat relative to the velocity profiles at $d=0.75$ " where flow acceleration has created a significant profile. The uniformity of the flow at $d=15.7$ " confirms that the flow was not significantly affected by the acceleration into the diffuser mouth.

Because the traverses at $d=0.75$ " span mostly across the opening to the diffuser mouth (see Figure 4.10), the velocity magnitudes are much higher. The velocity readings outside the lip 
of the diffuser, in the circulation region (for $\mathrm{y} / \mathrm{H}>1.5$ or $\mathrm{z} / \mathrm{W}>1$ ), could be negative velocity since hot film probes do not discriminate between positive and negative flow in the same coordinate direction.

The turbulence intensity profiles were calculated in the same manner as before. They are presented in Figure 5.10 and Figure 5.11 for $\theta=0^{\circ}$ and $90^{\circ}$, respectively.

In Figure 5.10, the $\theta=0^{\circ} \mathrm{Tu}$ profile for the high screen configuration can be seen to gradually increase from $6 \%$ turbulence intensity at $\mathrm{y} / \mathrm{H}=0$ to $12 \%$ at $\mathrm{y} / \mathrm{H}=3.4$. The $T u$ profile for the single screen configuration hovers between $2.5 \%$ to $3 \%$ turbulence from $y / H=0$ to $y / H=$ 1.3. For $\mathrm{y} / \mathrm{H}>1.5$, the turbulence intensity can be seen to be largely affected by the circulating flow as it increases drastically.

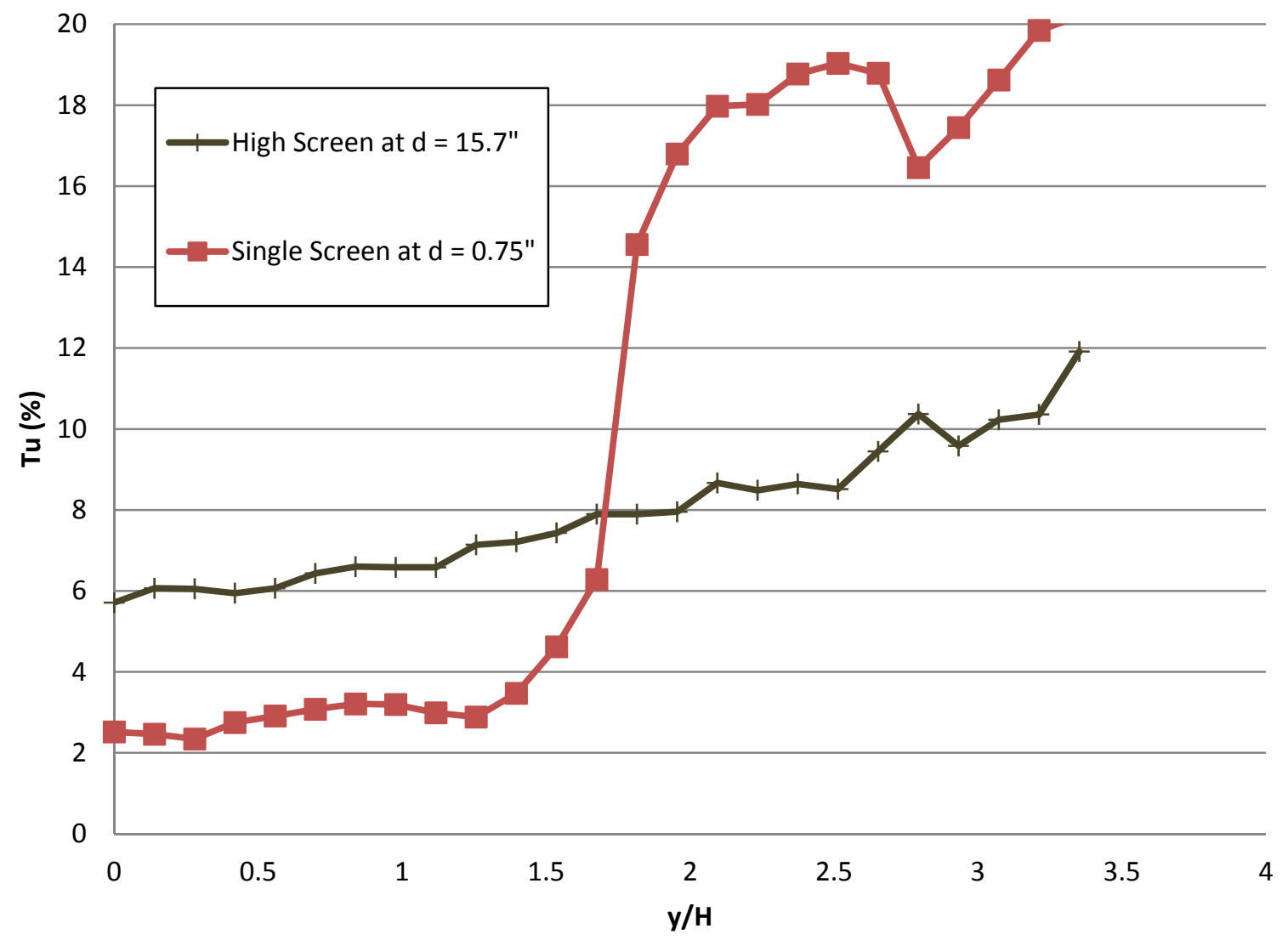

Figure 5.10: $T u$ profiles at $\theta=0^{\circ}$ (along $\mathrm{z} / \mathrm{W}=0$ ), for the high screen configuration. 
In Figure 5.11, the $\theta=90^{\circ} \mathrm{Tu}$ profile for the high screen configuration can be seen to gradually increase from $6.5 \%$ turbulence intensity at $\mathrm{y} / \mathrm{H}=0$ to $10 \%$ at $\mathrm{y} / \mathrm{H}=1.4$. The $T u$ profile for the single screen configuration hovers between $1.5 \%$ to $2.5 \%$ turbulence from $\mathrm{y} / \mathrm{H}=0$ to $\mathrm{y} / \mathrm{H}$ $=0.9$. For $\mathrm{y} / \mathrm{H}>1$, the turbulence intensity can again be seen to be largely affected by the circulating flow as it increases drastically.

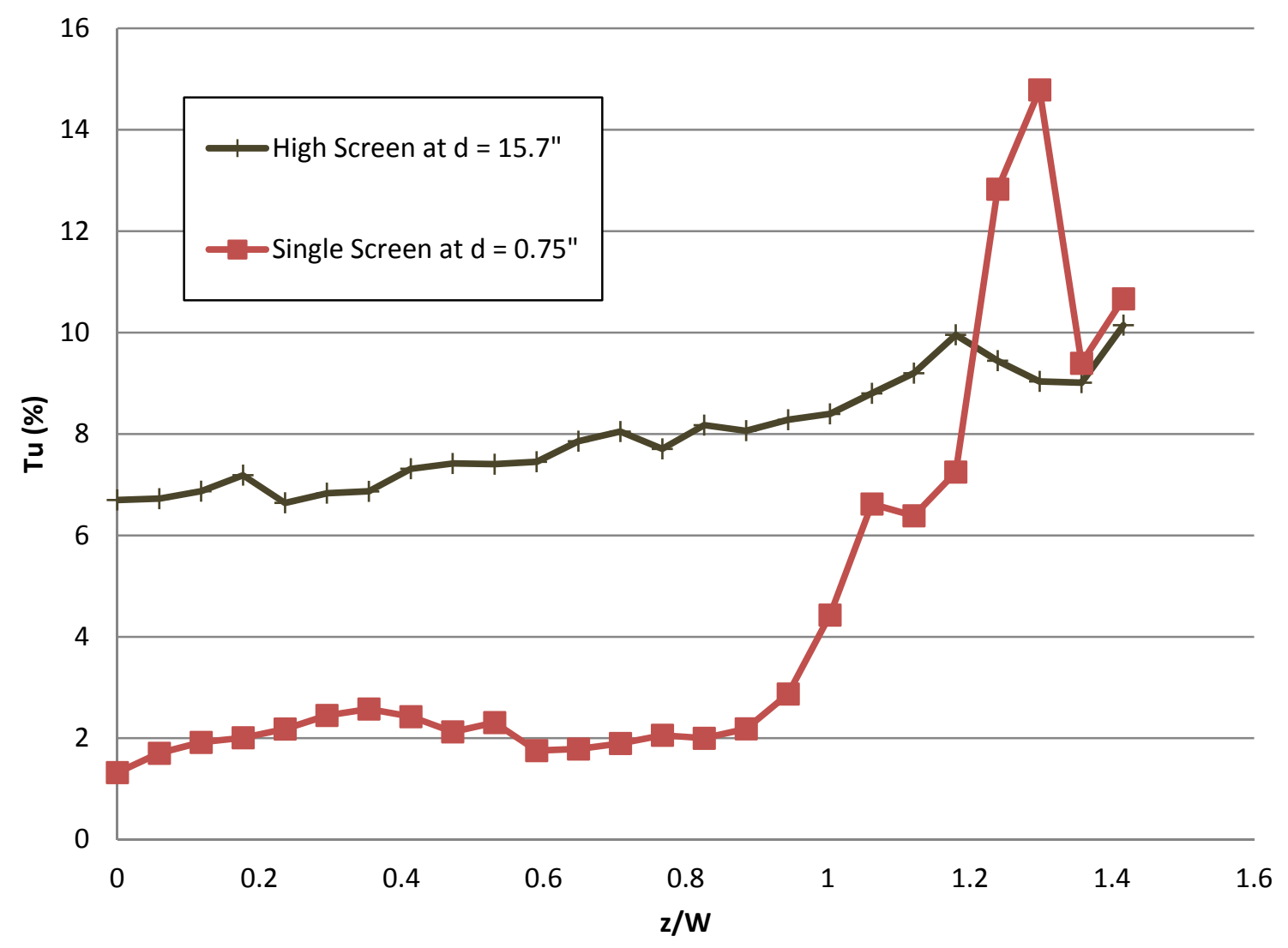

Figure 5.11: $T u$ profiles at $\theta=90^{\circ}$ for the high screen configuration.

Each of the high screen profiles is flat relative to the single screen profiles which rise rapidly outside of the diffuser lip (but are also relatively flat in front of the diffuser mouth). This suggests that the turbulence generated is relatively homogeneous, which has been proven to be characteristic of flow behind screens [16], [28]. 
In both traverses the average turbulence intensity across the profile for the high screen configuration was about $8 \%\left(7.9 \%\right.$ for $\theta=0^{\circ}$ and $\left.8.0 \% \theta=90^{\circ}\right)$. This value of turbulence intensity is very nearly in line with the prediction based on Roach's correlation. The average turbulence intensity across the profile for the single screen case at $\theta=0^{\circ}$ is $2.8 \%$ which is a $65 \%$ reduction in $T u$ even though measurements were taken 6.6 " closer to the turbulence screen. The average turbulence intensity across the profile for the single screen case at $\theta=0^{\circ}$ is $2.0 \%$ which is a $75 \%$ reduction in $T u$. Therefore these results confirm that flow acceleration considerably increases the rate of dissipation of turbulence intensity from what a steady flow experiences downstream of a turbulence grid.

\subsubsection{Off-Design Throat Mach Number Study}

The purpose of this study was to observe the effect of freestream turbulence on the total pressure recovery of a serpentine diffuser at varying throat Mach numbers. In this section, the inlet freestream turbulence for the distinct test configurations at varying throat Mach numbers is detailed. In section 5.3 the total pressure recovery at for the distinct test configurations at varying throat Mach numbers will be presented. As mentioned in Chapter 3, only the single screen and baseline cases were included in this experiment.

The velocity data at the center of the diffuser inlet versus Mach number for the off-design throat Mach number study is presented in Figure 5.12. Each of the profiles vary linearly from about $290 \mathrm{ft} / \mathrm{s}$ at Mach 0.55 to about $350 \mathrm{ft} / \mathrm{s}$ at Mach 0.725 . The purpose of presenting the velocity data for these two test configurations is to demonstrate that the mean conditions are reasonably equivalent, but the level of turbulence is what differentiates them. Indeed, the average velocities across each profile differ by only $1 \%$. 


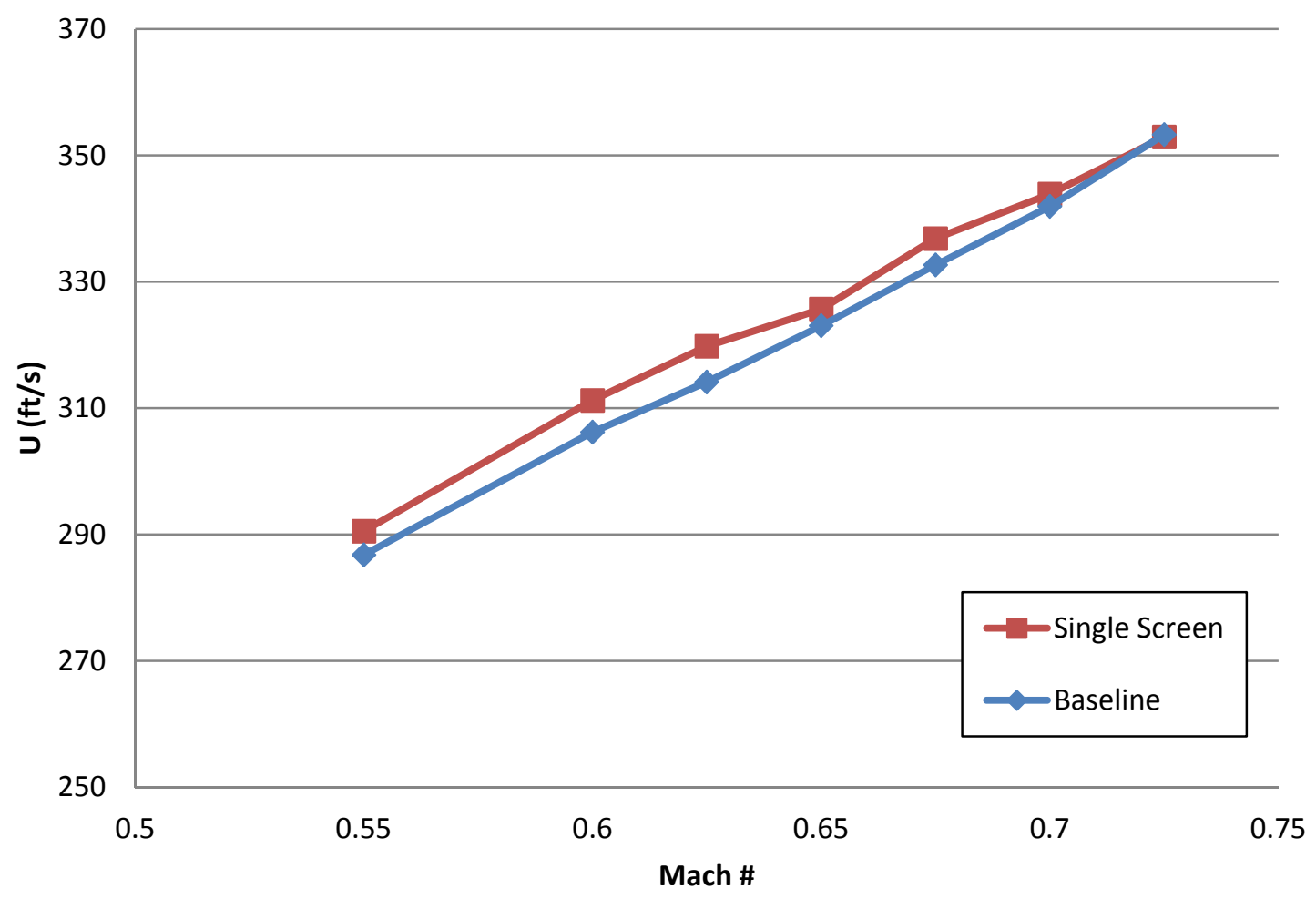

Figure 5.12: Mean velocity at $\theta=90^{\circ}, \mathrm{z} / \mathrm{W}=0.41, \mathrm{y} / \mathrm{H}=0.42$ with varying throat Mach number.

The turbulence intensity profiles computed from the data upstream of the diffuser inlet versus Mach number are presented in Figure 5.13. The turbulence intensity of the single screen configuration is about $2.4 \%$ at Mach 0.55 . It then decreases to $2 \%$ at Mach 0.625 and begins to rise until it reaches $2.3 \%$ at Mach 0.725 . The turbulence intensity of the baseline configuration is about $0.8 \%$ at Mach 0.55 and remains relatively stable until Mach 0.65 . It then rises from $0.8 \%$ turbulence intensity at Mach 0.65 to $1.3 \%$ at Mach 0.725 . As expected, the turbulence intensity is higher with the single screen configuration at every throat Mach number. Indeed the average turbulence intensity across the Mach number range for the single screen configuration represents a 132\% increase in turbulence intensity from that of the baseline configuration. 
However, the difference in turbulence intensity between the baseline and the single screen configurations narrows from $1.6 \%$ at Mach 0.55 to $1.1 \%$ at Mach 0.725 .

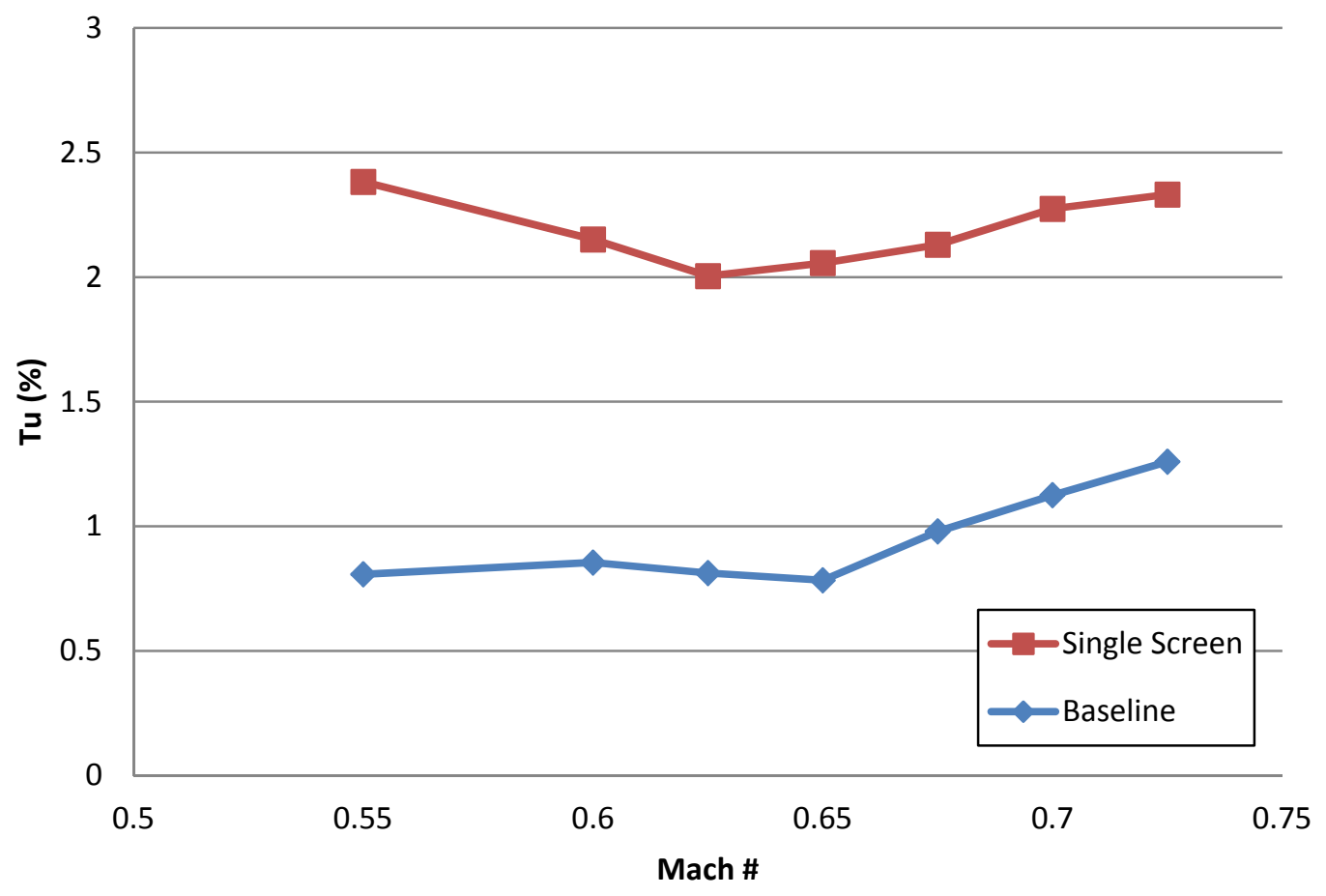

Figure 5.13: $T u$ at $\theta=90^{\circ}, \mathrm{z} / \mathrm{W}=0.41, \mathrm{y} / \mathrm{H}=0.42, \mathrm{~d}=0.75^{\prime \prime}$ with varying throat Mach number.

Interestingly, there seems to be a threshold where the turbulence intensity of the flow in both configurations starts to increase. The threshold appears to be at a throat Mach number of approximately 0.65 . In conclusion, the single screen configuration resulted in a higher level of inlet freestream turbulence than the baseline configuration across the range of diffuser throat Mach numbers.

\subsection{Axial Static Pressure Results}

In this section, the axial static pressure readings along the upper and lower surfaces of the diffuser will be presented and discussed. 
The axial wall static pressure data along the centerline of the lower surface are presented in Figure 5.14. The static pressure profiles for each configuration follow the same curvilinear trend. Starting at a static pressure of 9 psi at $\mathrm{x} / \mathrm{L}=0.05$, they rise to slightly over $13 \mathrm{psi}$ at $\mathrm{x} / \mathrm{L}=$ 0.45. The profiles then drop to $11.5 \mathrm{psi}$ at $\mathrm{x} / \mathrm{L}=0.75$ before hovering between $11.5 \mathrm{psi}$ and 12 psi until x/L $=1$.

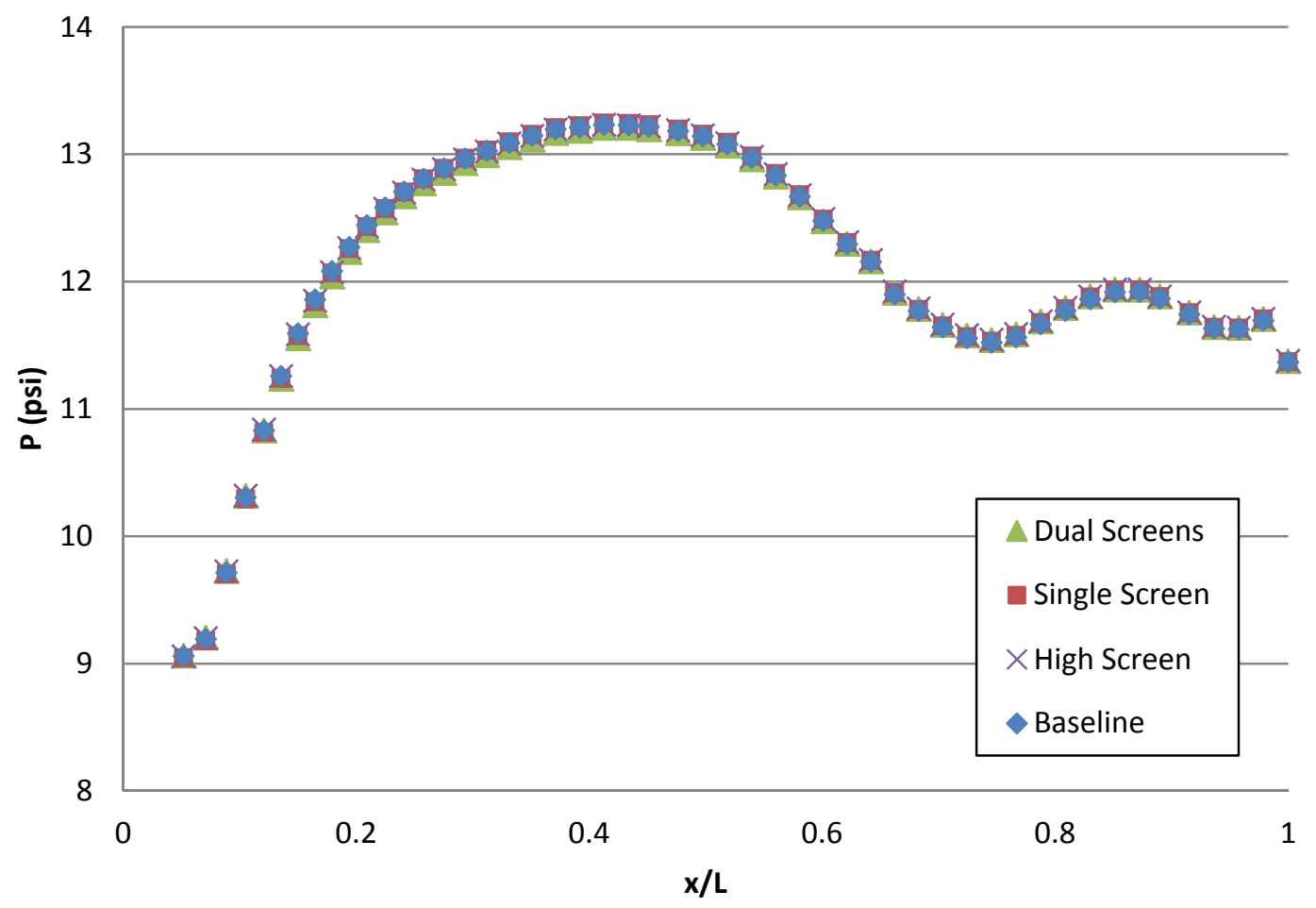

Figure 5.14: Axial static pressures along lower surface of diffuser.

The varying levels of turbulence seemingly had little effect on the static pressure profile along the lower surface of the inside of the serpentine diffuser. The static pressure profile of the dual screens configuration is noticeably lower from $\mathrm{x} / \mathrm{L}=0.2$ to $\mathrm{x} / \mathrm{L}=0.6$. This becomes slightly more apparent in Figure 5.15 where the same plot is rescaled to demonstrate the difference. The difference is roughly equivalent to the repeatability margin of \pm 0.03 psi. 


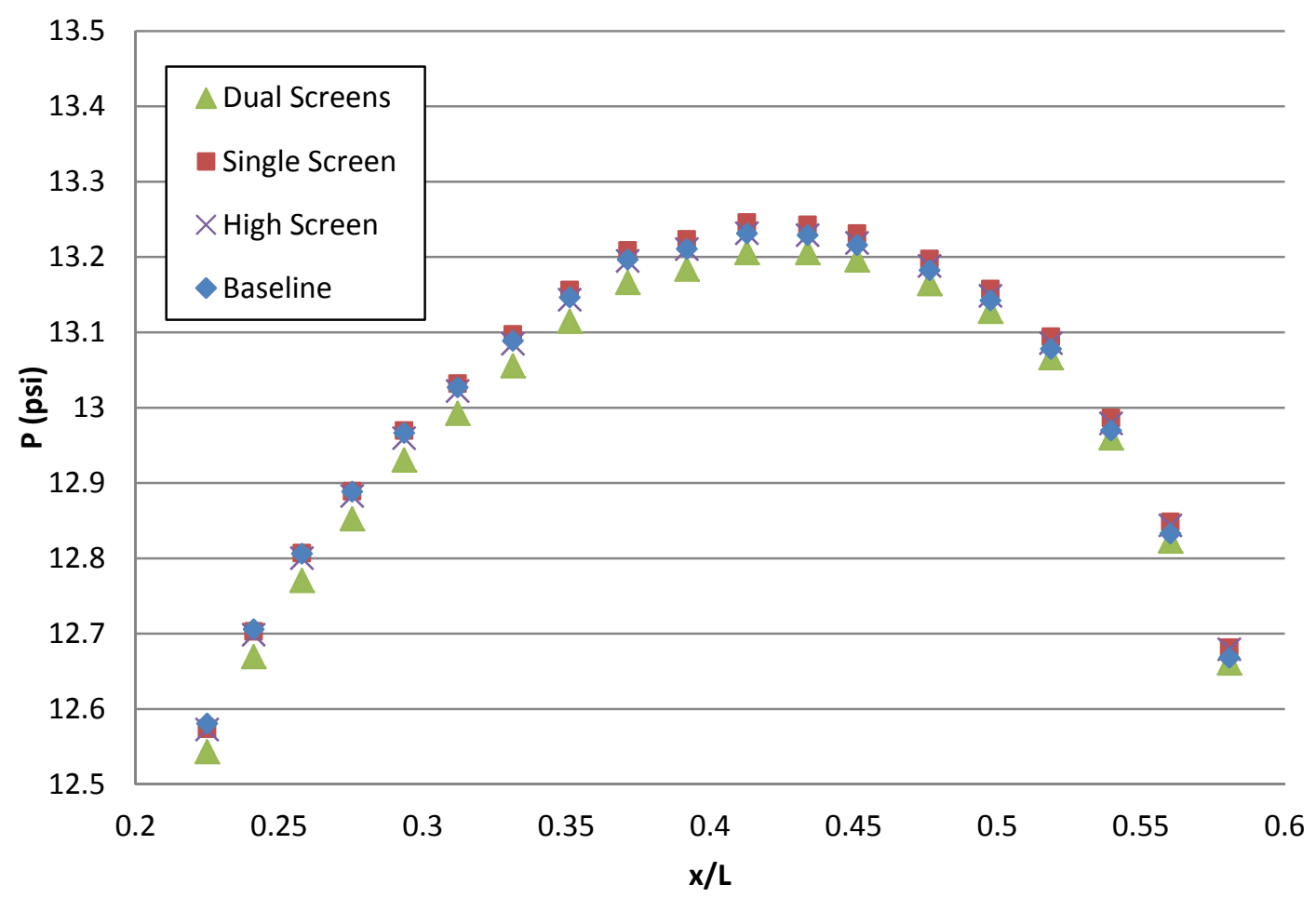

Figure 5.15: Rescaled axial static pressures along lower surface of diffuser.

The data for the axial wall static pressure along the centerline of the upper surface of the serpentine diffuser are presented in Figure 5.16. Again, the static pressure profiles for each test configuration are very similar. Initially they increase from 12.75 psi to 13 psi at $\mathrm{x} / \mathrm{L}=0.15$. Then the profiles decrease sharply to $9 \mathrm{psi}$ at $\mathrm{x} / \mathrm{L}=0.4$. They then increase sharply again to just over 11 psi at $\mathrm{x} / \mathrm{L}=0.5$. During this sharp rise, some of the profiles noticeably begin to differentiate themselves. Between $\mathrm{x} / \mathrm{L}=0.5$ and $\mathrm{x} / \mathrm{L}=0.6$, the pressure profile levels off then increases at a slower rate to nearly $12 \mathrm{psi}$. This discontinuity in the rise of the profile is despite the linear area divergence that is taking place. The leveling off of pressure indicates that flow separation is taking place which causes flow blockage and prevents the flow area from increasing. There is a gap in the measurements from just over $\mathrm{x} / \mathrm{L}=0.6$ to $\mathrm{x} / \mathrm{L}=0.9$ due to 
hardware limitations. The static pressure from $\mathrm{x} / \mathrm{L}=0.9$ to $\mathrm{x} / \mathrm{L}=1.0$ decreases from 13.1 psi to 12.9 psi.

The upper surface of the inside of the serpentine diffuser is of significant interest to this study because of the separation region after the final turn (about $x / L=0.5$ ). In observing the data of the static pressure readings in this area it is apparent that the varying levels of inlet freestream turbulence is indeed having an effect on the static pressure profile. In order to better observe the effects more closely, Figure 5.17 provides a re-scaled chart of the same data.

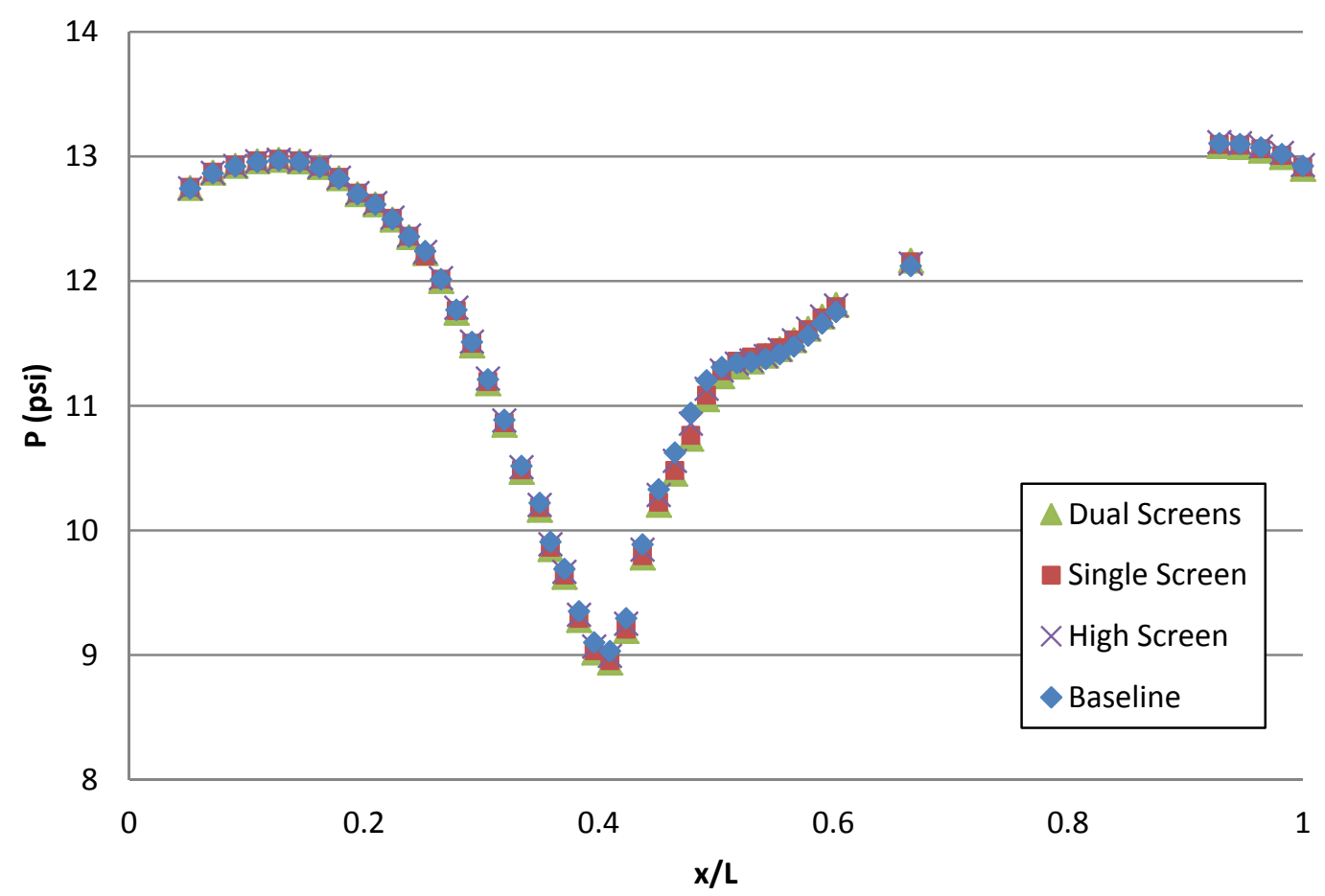

Figure 5.16: Axial static pressures along upper surface of diffuser.

From Figure 5.17, it is observed that the static pressure profiles for the test configurations with turbulence generation applied upstream of the diffuser mouth cross over the profile of the static pressures of the baseline configuration. This phenomenon in conjunction with the observation that the pressure profiles from each test configuration overlay each other reasonably 
well outside of the separation region eliminates the possibility that differences between data from different test configurations are a result of experimental setup biases. The measured difference between the profiles reaches up to $0.2 \mathrm{psi}$ for the dual screens and single screen case. This value is well beyond the repeatability, or precision, margin of \pm 0.03 psi.

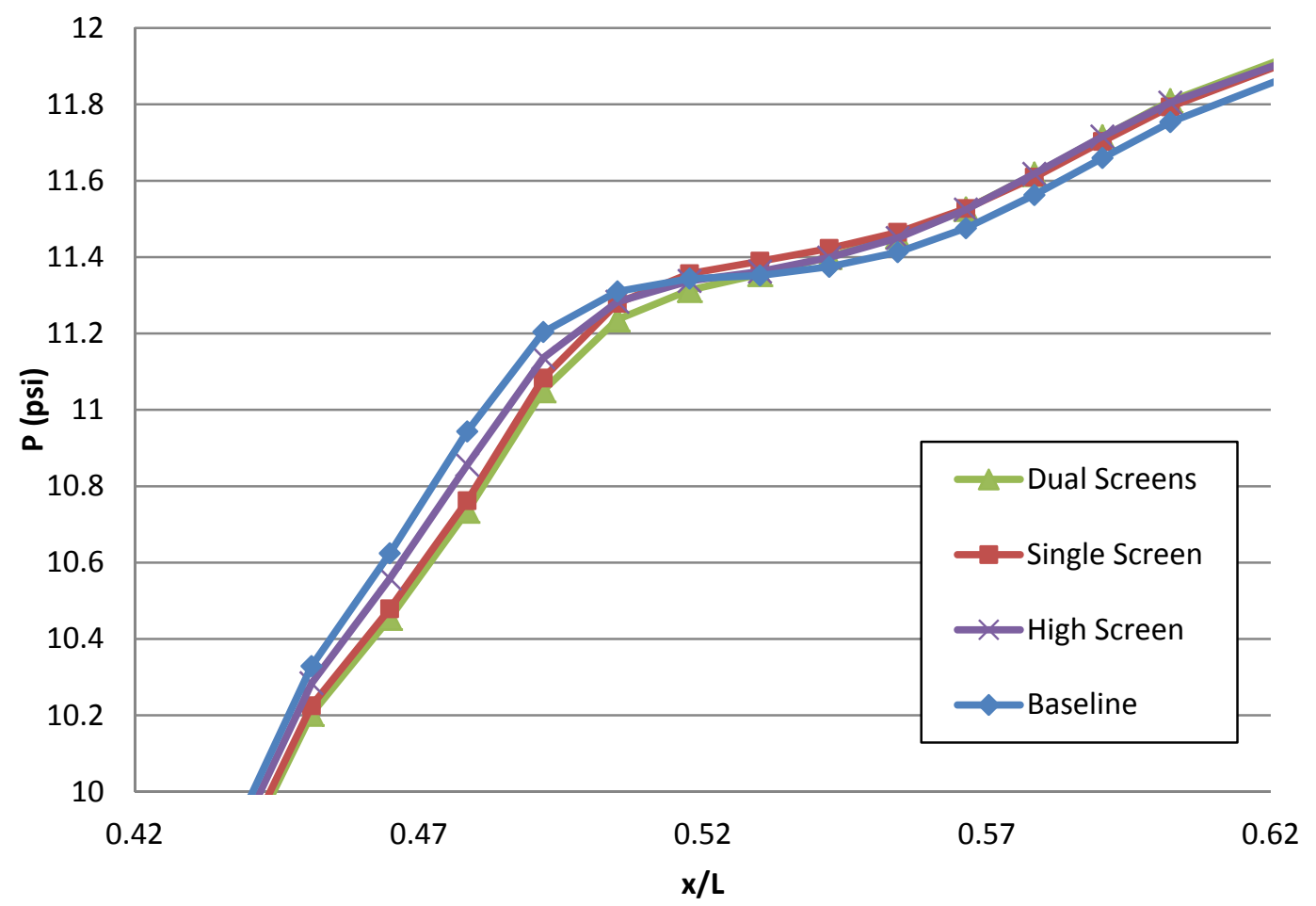

Figure 5.17: Re-scaled plot of the axial static pressures along upper surface of diffuser.

In order to better observe the difference between each of the test configurations with turbulence generation, an even more expanded view of the plot of the same data is provided in Figure 5.18. In each of the test configurations where turbulence is being generated upstream of the inlet to the serpentine diffuser, the static pressure jump in the separation region is noticeably less pronounced. In other words, the discontinuity in pressure rise that occurs due to flow separation after the second turn of the serpentine diffuser is less severe with higher levels of turbulence. This suggests that an increase in inlet freestream turbulence slightly reduces the 
magnitude of the flow separation that is occurring. The differences in static pressure between the turbulence configurations and the baseline are presented in Figure 5.19.

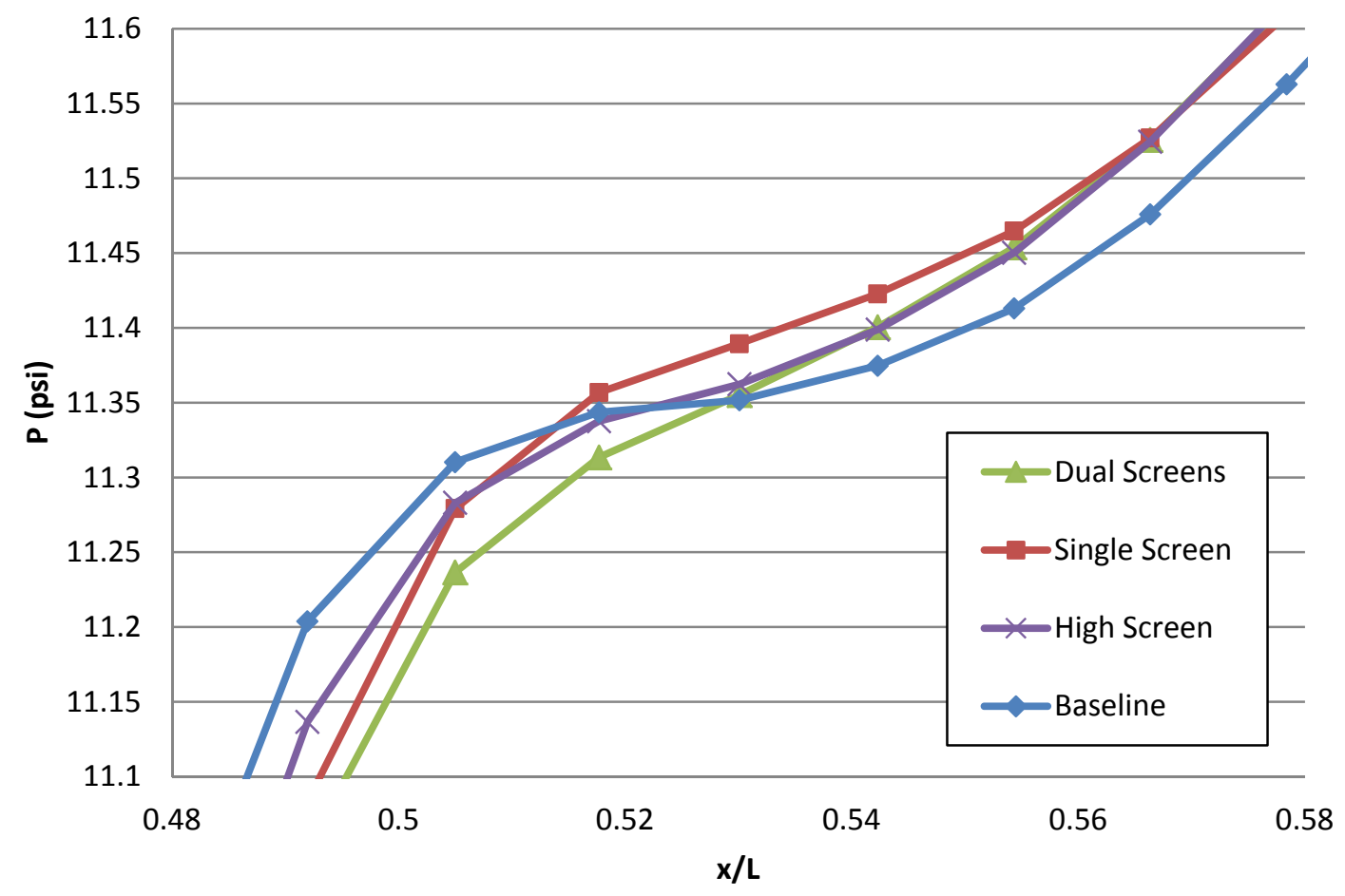

Figure 5.18: Further expanded view of axial static pressures along upper surface of diffuser.

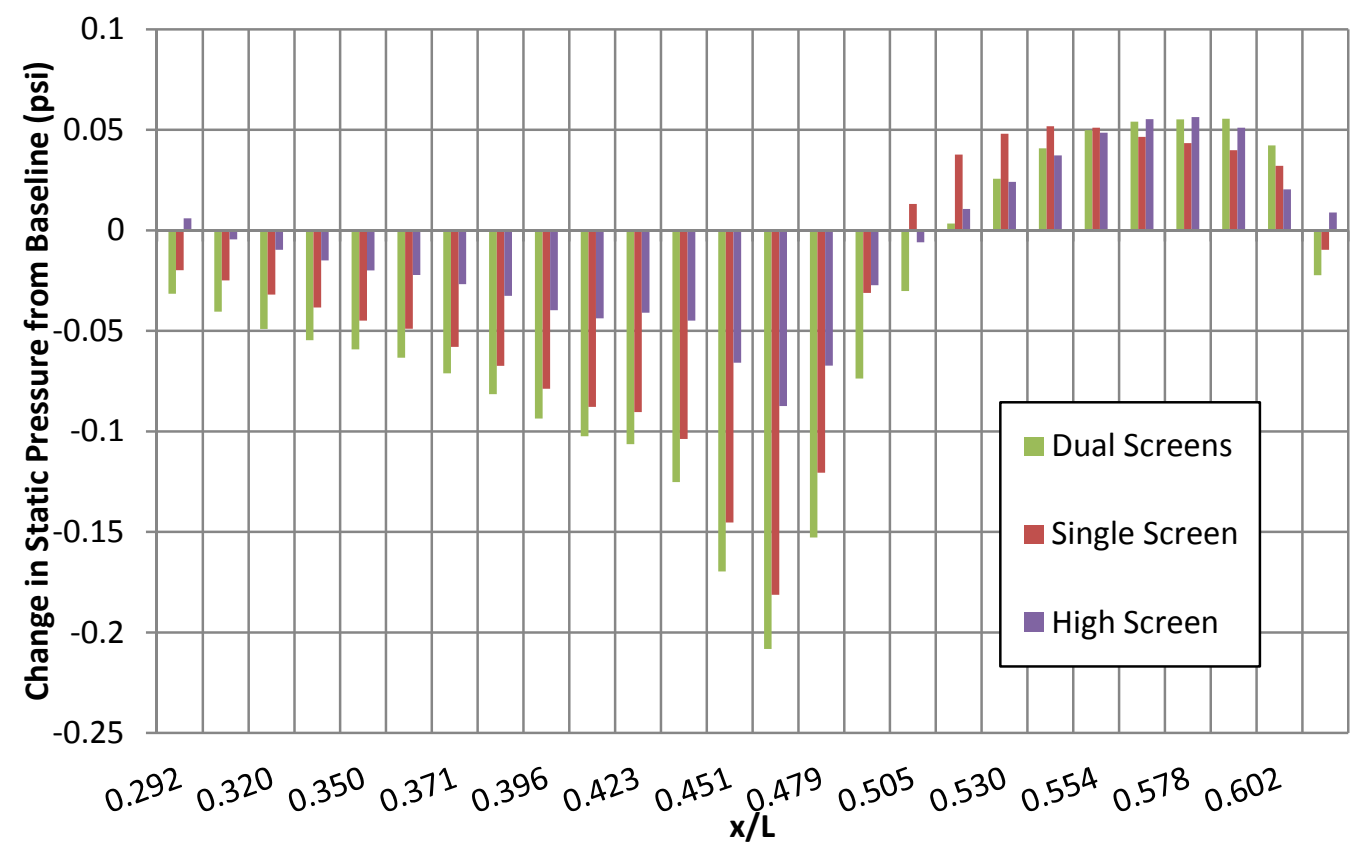

Figure 5.19: Difference in axial static pressure between turbulence cases and baseline case 
In Figure 5.19 it can be seen that the dual screens configuration results in a difference of static pressure that is 0.21 psi lower than the baseline case before the separation region, whereas the single screen configuration profile reaches a 0.18 psi difference and the high screen configuration profile reaches a difference of 0.09 psi. After the separation region, each of the profiles of the turbulence configurations level out to about 0.05 psi higher than the baseline profile.

The separation that occurs after the second turn of a serpentine diffuser is most significant culprit in flow distortion at the AIP. Hence, significant changes in this separation indicate that the elevated freestream turbulence is affecting the distortion patterns produced by the serpentine diffuser. The static pressure results presented in this section demonstrate a significant decrease in discontinuity in the flow separation region suggesting that freestream turbulence reduces the magnitude of separated flow past the second turn of a serpentine diffuser.

\subsection{AIP Total Pressure Results}

In this section, the total pressure measurements that were taken at the Aerodynamic Interface Plane (AIP), or exit of the diffuser (see Figure 1.1), are presented and discussed. In addition, the calculated total pressure recovery factor for each case are presented and discussed. The total pressure recovery values for the single screen are compared against those of the baseline at off-design throat Mach numbers.

\subsubsection{AIP Total Pressure Fields}

The AIP total pressure fields for each configuration are presented in Figure 5.20, Figure 5.21, Figure 5.22 and Figure 5.23. As discussed in Chapter 3, these contours are interpolated 
from 720 different total pressure readings spaced equally over the cross section. The total pressure measurements are corrected based on a reference inlet total pressure, $P_{0}$, which is set to 14.7 psia.

The standard features of a typical AIP total pressure field of a serpentine diffuser are all present for each case. The most notable is the total pressure deficit on the upper surface which is caused by the flow separation that occurs in the final turn of the serpentine diffuser. Separated flow and vorticity are a result of pressure gradients and high shear stresses. These high shear stresses also result in total pressure losses. Therefore, these total pressure data are indicative of the level of vorticity at the AIP. It can be observed that increasing the freestream turbulence did not eliminate this separated flow, although there are noticeable differences between the elevated freestream turbulence cases and the baseline case.

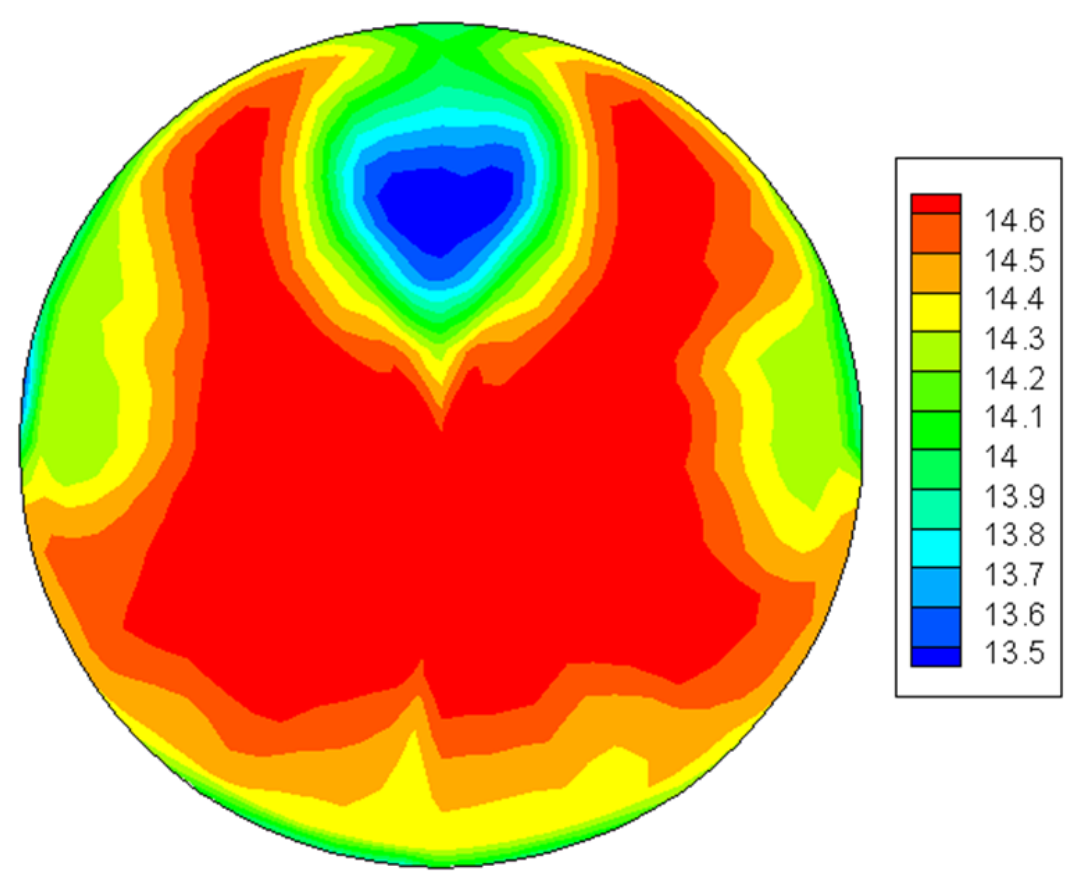

Figure 5.20: Baseline configuration AIP total pressure field (psi). 


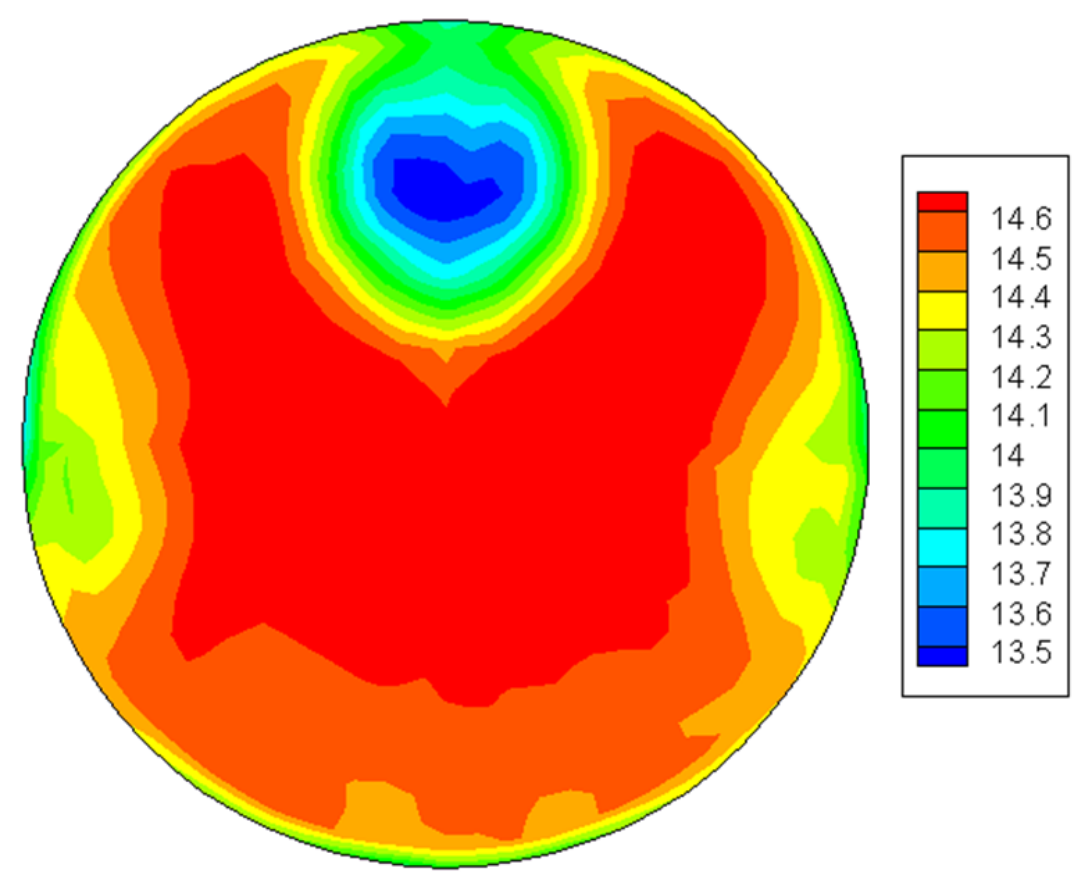

Figure 5.21: Single screen Configuration AIP total pressure field (psi).

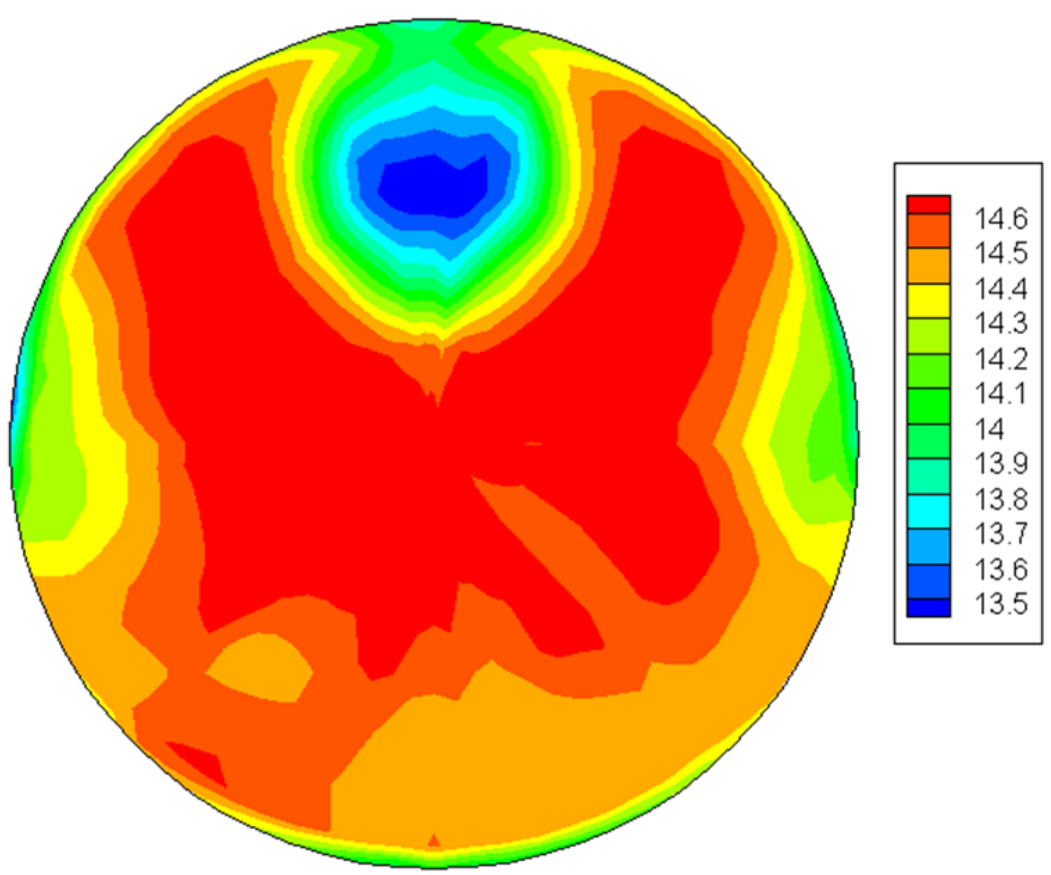

Figure 5.22: Dual screens configuration AIP total pressure field (psi). 


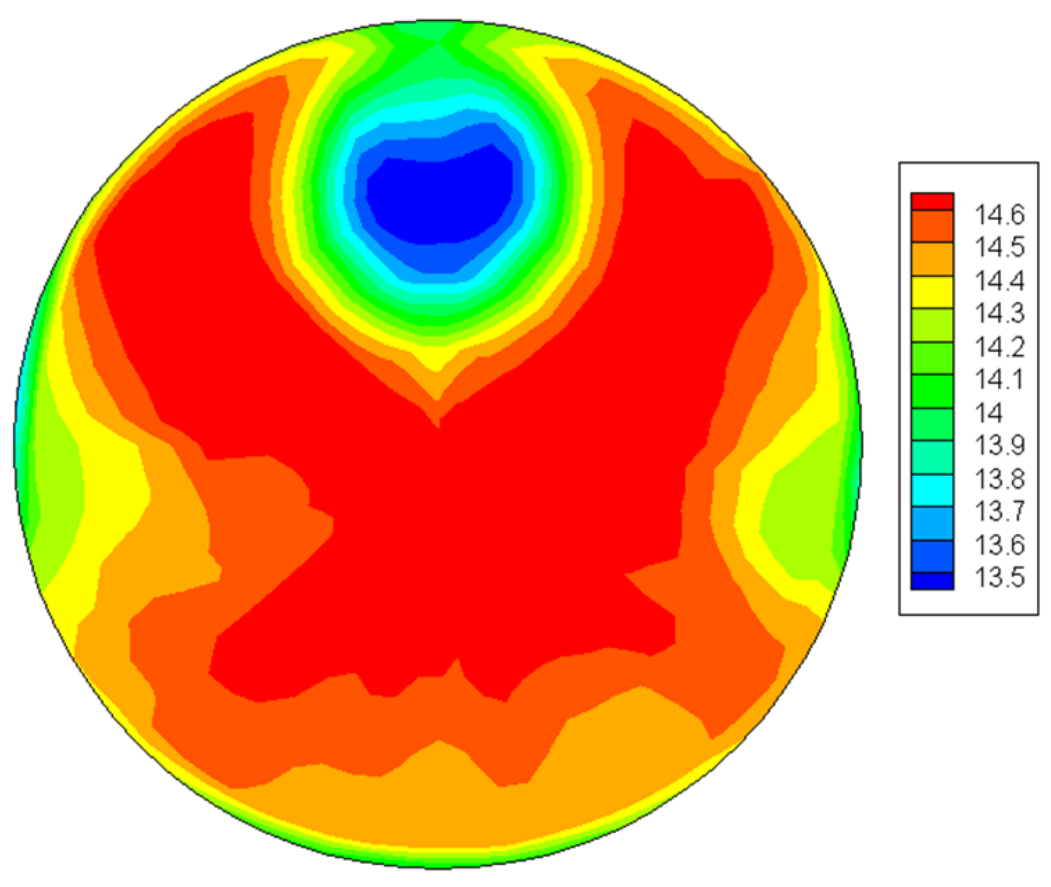

Figure 5.23: High screen configuration AIP total pressure field (psi).

The side wall vortices are also present in each case, although they are of a different shape than the side wall vortices that were computationally predicted as shown in Chapter 3 . The increase in freestream turbulence can be seen to slightly alter the size, shape and magnitude of the side wall vortices on the total pressure recovery.

Perhaps the most significant effect of the freestream turbulence on the AIP total pressure field is on the total pressure gradient towards the bottom of the AIP. In the baseline AIP field, this gradient is evident where the total pressure is low, less than 14.4 psi, along the lower surface. In the total pressure fields for the single screen configuration, the total pressure is higher than $14.5 \mathrm{psi}$ for most of the region. Although the thickness of this gradient (the distance from the surface to the maximum total pressure of the free stream) seems to have increased for the elevated turbulence configurations. Interestingly, the axial static pressure measurements along the lower surface of the diffuser, shown in Figure 5.14, gave no indication that the 
elevation in freestream turbulence had had an effect as compared to the baseline configuration. It does appear from the total pressure fields though that the pressure right on the surface is unaffected by varying levels of inlet freestream turbulence.

Although there were observable effects of freestream turbulence on the total pressure distortion field, the freestream turbulence clearly did not completely erase the serpentine inlet induced distortion. The low-mean velocity located top-center remains a primary concern for each configuration, as well as the side-wall vortices. In other words, the effects of the shape of the diffuser on the flow patterns remain a major concern despite the turbulence properties of the flow upstream of the diffuser. In order to better investigate the difference in total pressure recovery between the cases with turbulence generation and the baseline, contours of those differences were generated and are presented in Figure 5.24, Figure 5.25 and Figure 5.26.

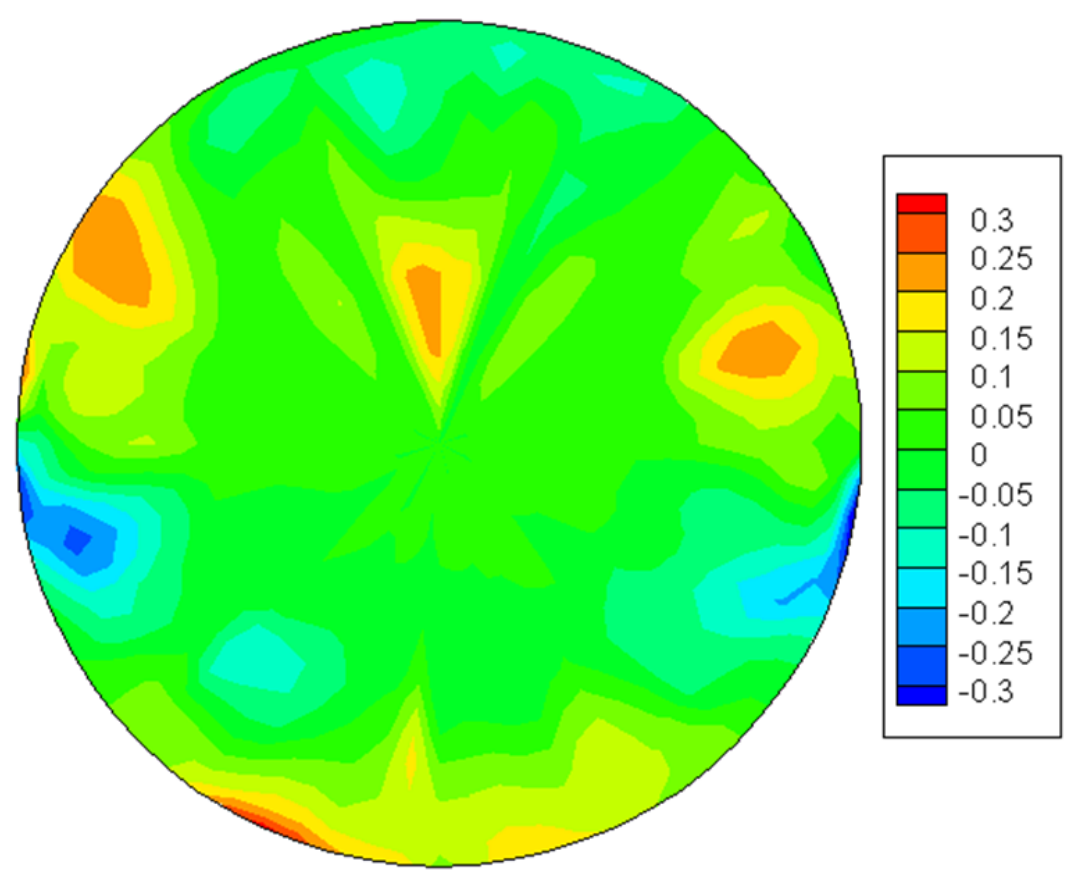

Figure 5.24: Total pressure difference between single screen and baseline cases (psi). 


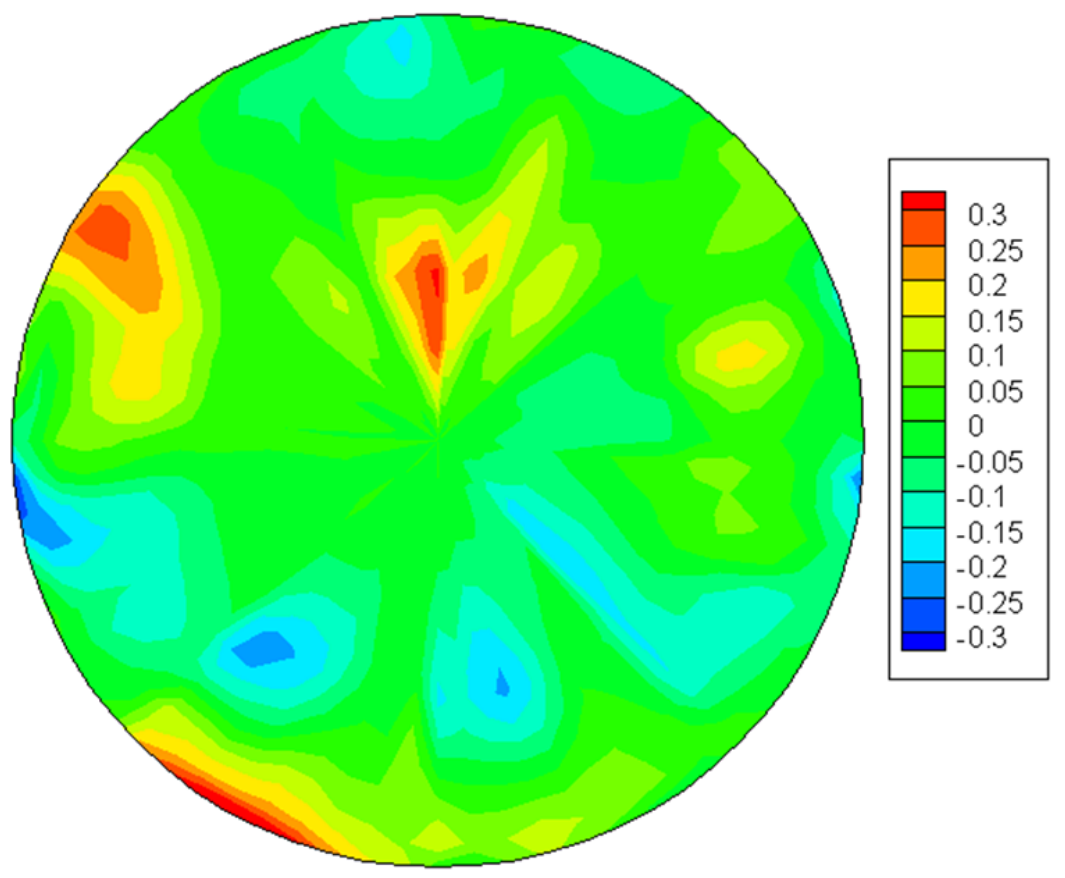

Figure 5.25: Total pressure difference between dual screens and baseline cases (psi).

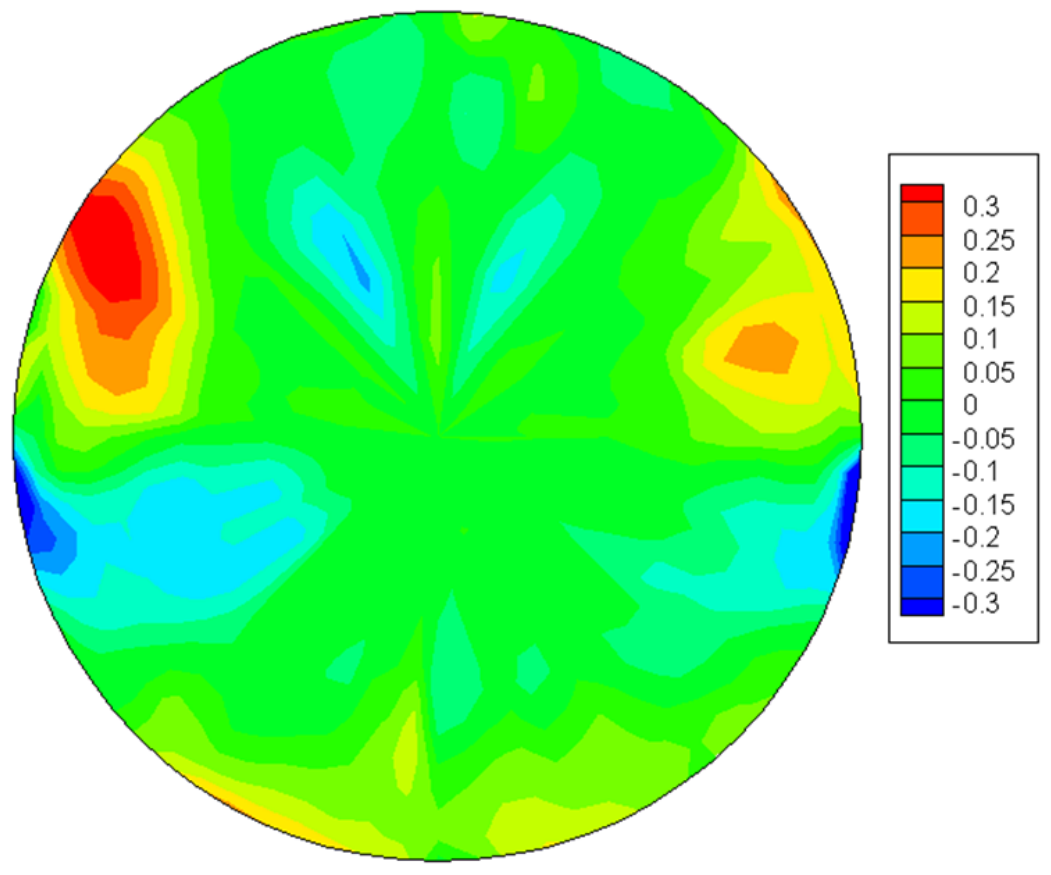

Figure 5.26: Total pressure difference between high screen and baseline cases (psi). 
An increase in total pressure recovery is present in the separated, low-mean velocity flow near the top. This was expected because of the effects seen on the flow separation from the axial static pressure measurements. This increase in recovery reaches as high as $0.2 \mathrm{psi}$ in the single screen configuration, and as high as 0.3 psi for the dual screen configuration. Not surprisingly, the recovery in this region for the high screen configuration is less than for the single screen configuration at a maximum of $0.1 \mathrm{psi}$. There are even some negative effects in this region with the high screen configuration.

The increase in recovery is also apparent in the total pressure gradient in the lower region of the AIP. The recovery in this region increases from the single screen configuration at up to $0.25 \mathrm{psi}$ to the dual screen configuration at up to $0.30 \mathrm{psi}$. The high screen total pressure field exhibits the smallest recovery in this region at up to 0.20 psi. The expansiveness of the recovery is larger for the single screen configuration than for either of the other two turbulence screen configurations.

The effects seen directly in the side wall vortices are negative. The dual screen case shows the smallest negative effect at $0.20 \mathrm{psi}$, followed by the single screen case at $0.25 \mathrm{psi}$ with the high screen cases demonstrating the highest negative effect over 0.30 psi. The negative effects seen in the side wall vortices are as significant in magnitude as the positive effects seen in the separated, low-mean velocity region.

There are, however, positive recovery effects just above the side wall vortices for each case. Comparing the effects across the three cases does not yield the same conclusion, that increasing the inlet freestream turbulence increases the total pressure recovery at the AIP. Unexpectedly, the high screen case, which exhibited the lowest level of inlet freestream turbulence aside from the baseline case, actually demonstrates the highest level of increase in 
total pressure recovery in these regions above the side wall vortices. It is unclear why the high screen configuration resulted in the highest recovery in this region. The dual screen configuration resulted in a higher recovery on the left side than the single screen configuration, but a lower recovery on the right side.

\subsubsection{AIP Total Pressure Recovery}

The dual screen configuration results in a decrease in pressure recovery up to 0.2 psi in a few regions above the lower region. This effect is barely noticeable in the other two turbulence configurations. This results in lowering the overall total pressure recovery as shown in Figure 5.27. The value $P_{t}$ is calculated from an average of 720 distinct total pressure readings that are spaced out across equal-area regions.

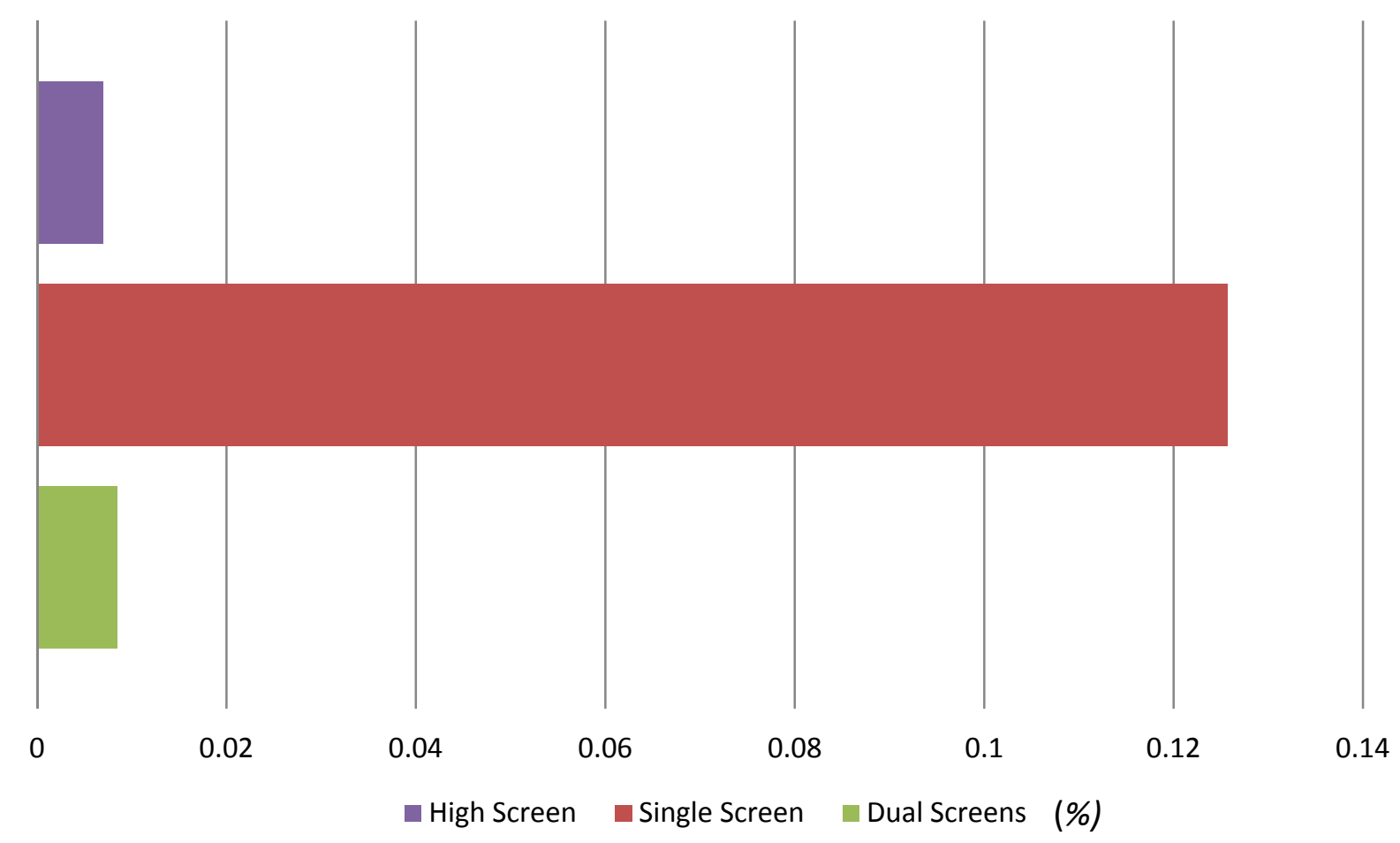

Figure 5.27: Total pressure recovery percentage increase from baseline for each test configuration. 
The total pressure recovery is slightly higher for each of the turbulence cases in comparison to the baseline case. The single screen turbulence configuration produced a more significant difference in pressure recovery at nearly $0.13 \%$ than either of the other two turbulence configurations. Both the total pressure recoveries of the dual screens configuration and the high screen configuration represent an increase of less than $0.01 \%$ beyond that of the baseline indicating that their negative effects on total pressure recovery are equal in magnitude to the positive effects.

The value changes in total pressure in the localized regions that have been discussed are well beyond the precision margin of \pm 0.02 psi. However, in calculating the overall total pressure recovery, the differences in each case are so small that they fall well within the precision margin. Additional AIP total pressure plots in Appendix D help to visualize the positive and negative effects that each turbulence configuration produces.

\subsubsection{Off-Design Mach Number Total Pressure Recovery}

Finally, the off-design diffuser throat Mach number results of the total pressure recovery factor are presented in Figure 5.28. The total pressure recovery factor in Figure 5.28 is based on the average of only 80 total pressure readings at each Mach number. As expected, the pressure recovery achieved with the single screen test configuration exceeds that of the baseline configuration across the range of throat Mach numbers. Although it is not an observably consistent trend with the amount of data available, the gap appears to slightly widen with increasing throat Mach number. The difference in total pressure recovery at Mach 0.55 is 0.0009 which increases to 0.0016 at Mach 0.725 . Hence, the positive effect on total pressure recovery due to an increase in freestream turbulence increases with increasing throat Mach number. 


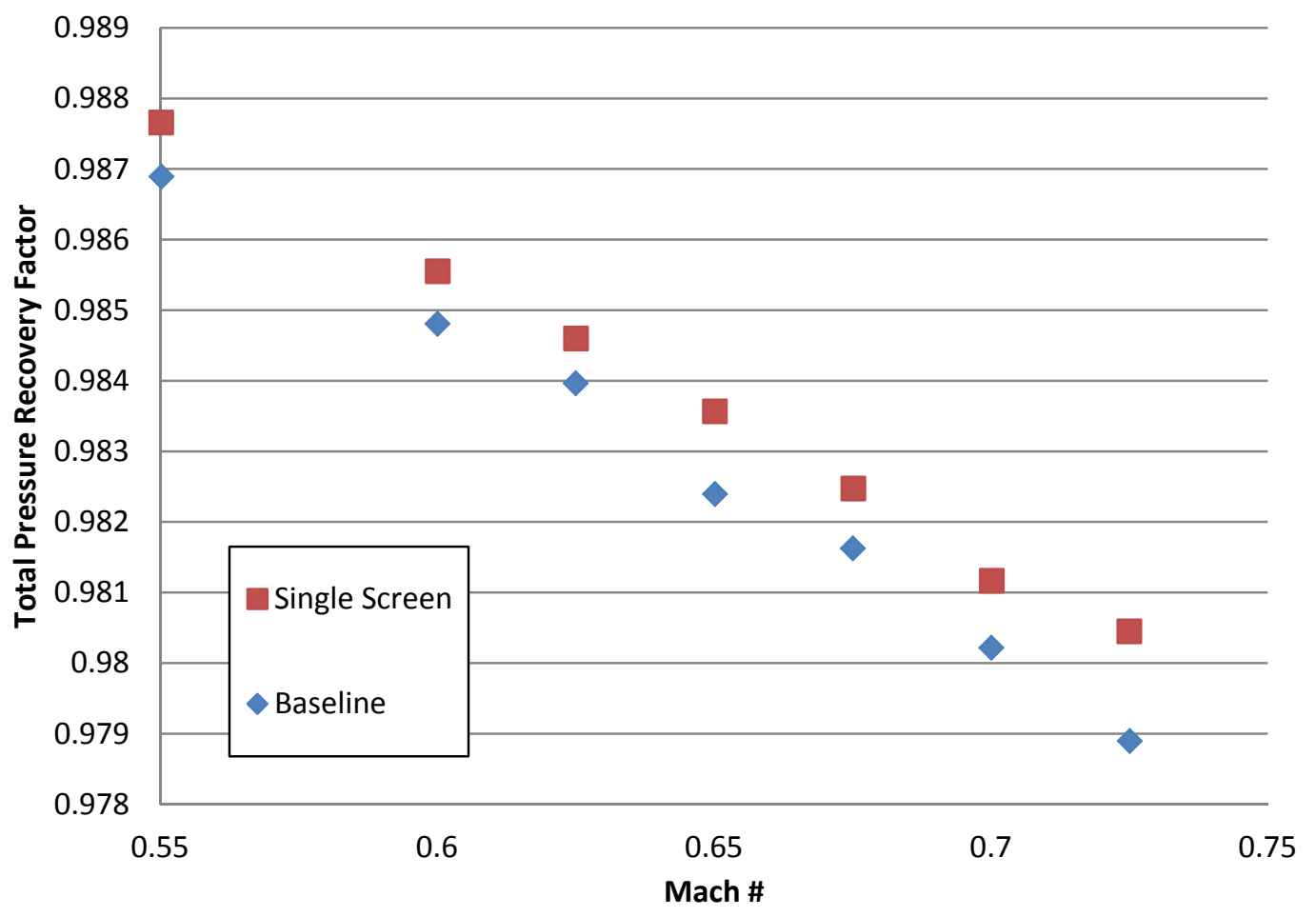

Figure 5.28: Total pressure recovery at off-design diffuser throat Mach numbers.

This study is important because not all aircraft employing serpentine diffusers will be designed for the same flight speed and hence the diffuser throat Mach number. The results from the off-design Mach number study demonstrate that the inlet freestream turbulence had an increasing effect on the total pressure recovery at the AIP of a serpentine diffuser across all throat Mach numbers that were tested. This effect becomes more significant at higher diffuser throat Mach numbers.

\subsection{Discussion of Significance of Total Pressure Recovery Results}

The single screen test configuration resulted in an increase of $0.12 \%$ in total pressure recovery across the AIP. In order to clarify the significance of this increase, a brief background 
review of two studies that investigated total pressure recovery of a serpentine diffuser is presented in this section.

In a study performed jointly by Lockheed Martin and the NASA Glenn Research Center, the effectiveness of certain Active Inlet Flow Control (AIFC) techniques was investigated [30]. The objective of AIFC is to mitigate the flow distortion and loss of total pressure recovery that occurs within a serpentine diffuser. The AIFC techniques that were used in this study were arrays of microvane vortex generators (VGs) and micro air-jet VGs. The idea behind these techniques is to generate vortices that are opposite in rotational direction but similar in magnitude to the vortices that are generated in the serpentine diffuser due to extreme wall curvature. In an experiment, the microvane technique resulted in a total pressure recovery increase of $5 \%$ at the AIP of the diffuser was achieved. The micro air-jet technique achieved a total pressure increase of $3 \%$ above that of the baseline.

Another study investigated the potential increase in total pressure recovery of a serpentine duct through optimization of the shape of the duct [31]. Using an optimization method known as the efficient global optimization (EGO) method the shape of a typical serpentine duct was altered with the objective of increasing total pressure recovery. Using CFD to analyze the total pressure recovery, it was found that the optimization of the serpentine duct resulted in a total pressure increase of up to $1.4 \%$ with identical inlet conditions.

Increasing the freestream turbulence resulted in an increase in total pressure recovery of a serpentine inlet that is an order of magnitude smaller than simply optimizing the serpentine inlet shape without altering inlet conditions $(0.12 \%$ compared to $1.4 \%$, respectively). Increasing inlet freestream turbulence is wholly unsuited as an AIFC technique since other methods such as vortex generation have already proven to be considerably more effective. Additionally, these 
findings suggest that small variations in inlet freestream turbulence will have a negligible effect in future AIFC studies on serpentine diffusers.

\subsection{Comparison of CFD and Experimental Results}

The experimental results of this study disagree with the general findings of the CFD analysis regarding the effects of freestream turbulence on serpentine diffuser performance patterns. The experimental results demonstrate that increasing the freestream turbulence upstream of the diffuser does have a significant effect on the pressure recovery in the low-mean velocity, separated flow near top dead center of the AIP, whereas the CFD predicted no effect. The experimental results also exhibited a significant effect on the total pressure recovery in the boundary layer near the bottom of the AIP whereas the CFD again predicted no effect. While the CFD model predicted an effect on the side wall vortices, it was much smaller in magnitude than the effect that was experimentally measured. It appears as though the CFD model had trouble computing turbulence near the wall which is where turbulence would have its greatest effect on the distortion patterns. In short, the CFD analysis failed to predict the effect of freestream turbulence on serpentine diffuser performance that was experimentally measured. 


\section{CHAPTER 6. CONCLUSIONS}

The serpentine diffuser presents an interesting and unique problem in the advancement of aircraft technologies. In addition to providing benefits that could not otherwise be achieved, serpentine diffusers introduce engine operability and efficiency issues. Consequently many studies, including this one, have been performed to investigate the effects of the shape of the serpentine diffuser on the flow physics of the air passing through it and its subsequent effects on the aircraft engine. The objective of this study was to investigate the effects of varying levels of inlet freestream turbulence on certain aspects of the flow physics of air that passes through a serpentine diffuser. Serpentine diffusers are a relatively new concept in the aviation industry. Although many studies have documented the behavior of air flow through a serpentine diffuser, the investigation of the effects of freestream turbulence on the distortion patterns induced by serpentine diffusers in this study is unprecedented. In order to accomplish this investigation, a method to generate turbulence was investigated and employed in the form of a square array of square and round bar grids. Experiments were executed using the ACF of the CARL facility at the AFRL. The findings from this study contribute to the research being performed at the AFRL on fan/inlet interaction with this particular serpentine diffuser.

Using three distinct turbulence screen configurations, turbulence intensities were increased from those of the baseline case by 0.5 to $2.5 \%$ in front of the diffuser mouth, up to nearly $4.0 \%$ turbulence intensity. The dual screens configuration resulted in the highest increase of turbulence intensity, 1.5 to $2.5 \%$, although not much higher than the single screen 
configuration with a 1.0 to $2.0 \%$ increase. The high screen configuration increased the turbulence intensity from the baseline case by 0.5 to $1.0 \%$. The measured turbulence intensity of the flow in front of the diffuser mouth in the baseline case ranges from 0.0 to $2.0 \%$.

In the absence of flow acceleration, the square bar turbulence grid produced a turbulence intensity profile with an average of about $8 \%$ (ranging from 6 to $10 \%$ ) at a distance of 21.6 " downstream. It previously was documented that the same square bar grid generated a freestream turbulence intensity of $2-3 \%$ at 15.0 " downstream in accelerating flow. This suggests that flow acceleration considerably increases the rate of dissipation of the turbulent kinetic energy of the flow. As far as the author is aware, this is the first study which has looked at the effect of flow acceleration on the dissipation of grid-generated turbulence.

The flow downstream of the single screen configuration maintained a higher turbulence intensity than that of the baseline case across the sweep of off-design throat Mach number tests, although the increase in turbulence intensity slightly narrows from about $1.5 \%$ at Mach 0.55 to $1.0 \%$ at Mach 0.725 .

The measured axial wall static pressure profile along the upper surface of the diffuser for each test configuration indicates that higher levels of freestream turbulence do have an effect on the flow separation that occurs after the final turn in the serpentine diffuser. Increasing the freestream turbulence upstream of the serpentine diffuser inlet has the effect of smoothing the increase in pressure that occurs in the region of separation. The pressure increase was progressively smoother with increasing levels of freestream turbulence (high screen, single screen, then dual screens). The difference in the static pressure profiles reached up to 0.2 psi for the single screen and dual screens configurations when compared to the baseline configuration. It is unsure what the significance is of the effect of freestream turbulence on the separation that 
occurs around the final bend of a serpentine diffuser from this data set alone, but the axial wall static pressure results do demonstrate that the flow behavior is being affected.

The measured total pressure field at the AIP demonstrated significant differences between cases with elevated inlet freestream turbulence and the baseline case. The dual screen test configuration resulted in an increase in the AIP total pressure recovery in the separation region of up to $0.3 \mathrm{psi}$, an increase of $25 \%$, whereas the single screen test configuration increased the AIP total pressure recovery by up to $0.2 \mathrm{psi}(17 \%)$. The high screen test configuration resulted in a net negative effect on the AIP total pressure recovery in the separation region. The total pressure recovery in the total pressure gradient near the lower surface at the AIP was increased by $0.30 \mathrm{psi}, 0.25 \mathrm{psi}$, and $0.20 \mathrm{psi}$ for the dual screens, single screen and high screen configurations, respectively. The elevated levels of freestream turbulence actually reduced total pressure recovery in the side wall vortices, although they each increased total pressure recovery in the regions above the side wall vortices.

The total pressure recovery coefficient for the entire AIP was slightly increased in each case of increased inlet freestream turbulence. The single screen test configuration resulted in the highest increase of total pressure recovery demonstrating an increase of 0.0012 ( or $0.13 \%$ ) above the total pressure recovery of the baseline, whereas the dual screens and high screen configurations resulted in a nearly insignificant increase in total pressure recovery at less than 0.0001 (less than $0.01 \%$ ). The turbulence screens installation results in pressure losses that are not present in the baseline case. The dual screens installation increases pressure losses by $50 \%$ when compared to the single screen configuration (see section 3.1.6) which mostly negates the reduction in distortion that is achieved by this test configuration. The high screen installation results in the same amount of pressure losses as the single screen configuration but exudes a 
lower level of inlet freestream turbulence. Hence the single screen test configuration results in the highest increase in total pressure recovery at the AIP.

The single screen configuration also resulted in a slightly higher total pressure recovery coefficient than the baseline across the range of off-design throat Mach numbers. The increase ranges from 0.0009 (or $0.09 \%$ ) at Mach 0.55 to 0.0016 (or $0.16 \%$ ) at Mach 0.725 . Hence the increase in total pressure recovery with a higher level of freestream turbulence improves with increasing diffuser throat Mach number.

The effects of freestream turbulence on serpentine diffuser distortion patterns are measurably significant, but minimal. Although they were affected, the distortion patterns were not eliminated. It is not necessary to mitigate varying levels of freestream turbulence to perform other types of research on the distortion patterns induced by serpentine inlets. Increasing the inlet freestream turbulence is not comparable to proven AIFC methods in the elimination or major reduction of the distortion patterns induced by serpentine diffusers.

The experimental results of this study were not in agreement with the predictions based on the CFD analysis. The CFD analysis exhibited no effect on the low-mean velocity separated flow near top dead center of the AIP and little effect on the side wall vortices. The experimental results showed significant effects in both of these regions of the AIP. In conclusion the $k-\omega S S T$ appears to be inadequate in predicting the effect of freestream turbulence on total pressure distortion patterns produced by serpentine inlets.

It is recommended for future work to investigate the effects of higher levels of freestream turbulence on serpentine diffuser distortion patterns to determine if there is a limit to the magnitude of the effect observed in this study. In order to accomplish this, it is likely necessary to use an active method of turbulence generation such as a grid with rotating vanes or secondary 
jets of air. Using secondary jets of air may also result in less pressure losses. Future researchers could also investigate generating turbulence immediately in front of the diffuser mouth to bypass the turbulence dissipation that occurs due to flow acceleration, although this would likely result in much higher pressure losses especially with a screen. 


\section{REFERENCES}

[1] Rabe, A., 2003. "Effectiveness of a Serpentine Inlet Duct Flow Control Scheme at Design and Off-Design Simulated Flight Conditions." PhD Thesis, Virginia Polytechnic Institute and State University.

[2] Farmer, C. J., 1972. "Inlet Distortion, Vorticity, and Stall in an Axial-Flow Compressor." Master's Thesis, Naval Postgraduate School.

[3] Yao, J., Gorrell, S., and Wadia, A., 2007. "A Time-Accurate Analysis of Inlet Distortion Induced Swirl in Multistage Fans.” AIAA 2007-5059, July.

[4] SAE International, 2002. "Gas Turbine Engine Inlet Flow Distortion Guidelines." ARP1420, Rev. B.

[5] Cousins, W. T., 2004. "History, Philosophy, Physics, and Future Directions of Aircraft Propulsion System / Inlet Integration.” ASME Paper GT2004-54210, June.

[6] Yao, J., Gorrell, S., and Wadia, A., 2010. "High-Fidelity Numerical Analysis of Per-RevType Inlet Distortion Transfer in Multistage Fans-Part I: Simulations With Selected Blade Rows." ASME Journal of Turbomachinery, 132, October, p. 041014.

[7] Scribben, A. R., Ng, W., and Burdisso, R., 2006. "Inlet Duct Flow Control Technique at Design and Off-Design Simulated Flight Conditions." Transactions of the ASME, 128, pp. 332-339.

[8] Kirk, A. M., Gargoloff, J. I., Rediniotis, O. K., and Cizmas, P. G. A., 2009. "Numerical and experimental investigation of a serpentine inlet duct." International Journal of

Computational Fluid Dynamics, 23(3), March, pp. 245-258.

[9] Harper, D. K., 2000. "Boundary Layer Control and Wall-Pressure Fluctuations in a Serpentine Inlet.” Master's Thesis, Virginia Polytechnic Institute and State University.

[10] Moore, C. A., Jr., and Kline, S. J., 1955. "Some Effects of Vanes and of Turbulence on Performance of Two-Dimensional Diffusers.” Department of Mechanical Engineering, Stanford University, September. 
[11] Waitman, B. A., Reneau, L. B., and Kline, S. J., 1961. "Effects of Inlet Conditions on Performance of Two-Dimensional Diffusers." Journal of Basic Engineering, 83, pp. 349360.

[12] Sajben, M., Chen, C. P., and Kroutel, J. C., 1977. “A New Passive Boundary Layer Control Device.” Journal of Aircraft, 14(7), July, pp. 654-660.

[13] Hoffman, J. A., 1981. "Effects of Free-Stream Turbulence on Diffuser Performance." Journal of Fluids Engineering, 103, September, pp. 385-390.

[14] Baines, W. D., and Peterson, E. G., 1951. "An Investigation of Flow Through Screens." Transactions of the ASME, July, pp. 467-480.

[15] Roach, P. E., 1986. "The generation of nearly isotropic turbulence by means of grids." Heat and Fluid Flow, 8(2), June, pp. 82-92.

[16] Roach, P. E. 1986. "The generation of nearly isotropic turbulence downstream of streamwise tube bundles." International Journal of Heat \& Fluid Flow, 7(2), June, pp. 117125.

[17] Makita, H., 1991. "Realization of a large-scale turbulence field in a small wind tunnel." Fluid Dynamics Research, 8, pp. 53-64.

[18] Mydlarski, L. and Warhaft, Z., 1996. "On the onset of high-Reynolds-number gridgenerated wind tunnel turbulence.” Journal of Fluid Mechanics, 320, pp. 331-368.

[19] Larssen, J. V. and Devenport, W. J., 2011. "On the generation of large-scale homogeneous turbulence.” Experiments in Fluids, 50, pp. 1207-1223.

[20] Ling, S. C. and Wan, C. A., 1972. "Decay of Isotropic Turbulence Generated by a Mechanically Agitated Grid." The Physics of Fluids, 15(8), pp. 1363-1369.

[21] Thole, K. A., Bogard, D. G., and Whan-Tong, J. L., 1994. "Generating high freestream turbulence levels." Experiments in Fluids, 17, pp. 375-380.

[22] Tassa, Y. and Kamotani, Y., 1975. "Experiments on turbulence behind a grid with jet injection in downstream and upstream direction.” The Physics of Fluids, 18(4), April, pp. 411-414.

[23] Pope, S. B., 2000. Turbulent Flows. Cambridge University Press, New York.

[24] Frenkiel, F. N., 1948. "The Decay of Isotropic Turbulence." Transactions of the ASME, 70, pp. 311-321.

[25] McQuilling, M. W., 2007. "Design and Validation of a High-Lift Low-Pressure Turbine Blade.” PhD Thesis, Wright State University, pp. 102-106. 
[26] Stalp, S. R., Skrbek, L. and Donnelly, R. J., 1999. "Decay of Grid Turbulence in a Finite Channel.” Physical Review Letters, 82(24), June, pp. 4831-4834.

[27] Laws, E. M. and Livesey, J. L., 1978. "Flow Through Screens." Annual Review of Fluid Mechanics, 10, pp. 247-266.

[28] Batchelor, G. K., 1953. The theory of homogeneous turbulence. Cambridge University Press, New York, (pp. 133-134).

[29] TSI Inc., 2008. TSI Thermal Anemometry Probes.

[30] Hamstra, J. W., Miller, D. N., Truax, P. P., Anderson, B. A., and Wendt, B. J., 2000. "Active Inlet Flow Control Technology Demonstration." International Council of the Aeronautical Sciences.

[31] Bae, H. G., Park, S. H., and Kwon, J. H., 2012. "EGO Method for Diffusing S-duct Shape Design." International Conference on Computation Fluid Dynamics, 7, July.

[32] Richardson, L. F., 1922. Weather Prediction by Numerical Process. Cambridge University Press, Cambridge.

[33] Kolmogorov, A. N., 1941. "The local structure of turbulence in incompressible viscous fluid for very large Reynolds numbers.” Dokl. Akad. Nauk SSSR, 30(4), pp. 299-303.

[34] Taylor, G. I., 1938. "The spectrum of turbulence." Proceedings of the Royal Society of London Series A, Mathematical and Physical Sciences, 164(919), February, pp. 476-490.

[35] Bernard, P. S., and Wallace, J. M., 2002. Turbulent Flow: Analysis, Measurement, and Prediction. John Wiley and Sons, Inc., Hoboken, New Jersey. 


\section{APPENDIX A. HOT WIRE ANEMOMETRY CALIBRATION}

This appendix provides information regarding the calibration technique for the hot film probes. The hot film probes were calibrated in a Model 1127 TSI Air Velocity Calibrator. The calibrator requires a supply of pressurized air to a nozzle that produces a relatively uniform jet of air. Coarse and fine adjustment valves are built into the calibrator to adjust the back pressure of the nozzle, and hence the air velocity, coarsely or finely, respectively. It also includes a stand to mount the hot film probe with its holder just downstream (above) of the jet of air. There is a pressure tap located on the outside of the plenum that is upstream of the nozzle. With a pressure transducer linked to this pressure tap, the plenum pressure can be measured which allows for calculation of the air jet velocity using a derivation of Bernoulli's principle:

$$
v=\sqrt{\frac{2}{\rho} * \Delta P}
$$

where $v$ is the air velocity, $\rho$ is the air density and $\Delta P$ is the difference between the plenum pressure and ambient pressure. The air is assumed to be stagnant in the plenum where the pressure tap is located. A possible source of error is introduced by using Bernoulli's principle to calibrate for velocity because the flow inside of the test facility reaches sufficiently high Mach number that incompressible flow cannot be assumed.

The hot film sensor that was used is a model $1263 \mathrm{~A}-10$, which is a $45^{\circ}$ angle to probe sensor. The probe alignment and CTA settings in the IFA-300 were kept the same from 
calibration to experiment. The CTA settings for the two sensors that were used are tabulated in Table A.1.

The gain was used to refine the resolution of the voltage measurement. This was especially important for higher velocity readings since the relation between voltage and velocity is exponential. The offset voltage was used to set the voltage close to zero for zero velocity and to ensure that the maximum voltage was not surpassed on the $\mathrm{A} / \mathrm{D}$ system. The Cable and Channel Resistances were measured using an internal function of the IFA 300. The optimal Operating Resistance is specified for each sensor by the manufacturer. The Operating Resistance can be adjusted to achieve better resolution at higher or lower velocities, but this was not explored in this study. Sensor 005005 (serial number) was used in the baseline and single screen experiments while sensor 005006 was used in the dual screens and high screen experiments.

Table A.1: CTA settings for the two hot film probes used in experiments.

\begin{tabular}{ccc}
\hline Setting & Serial \# 005005 & Serial \# 005006 \\
\hline Cable Resistance $(\Omega)$ & 1.61 & 1.61 \\
Channel Resistance $(\Omega)$ & 5.06 & 4.22 \\
Operating Resistance $(\Omega)$ & 8.38 & 8.13 \\
Cable Length $(\mathrm{m})$ & 30 & 30 \\
Gain & 4 & 4 \\
Offset Voltage $(\mathrm{V})$ & 1.28 & 1.45 \\
Low-Pass Filter $(\mathrm{kHz})$ & 20 & 20 \\
\hline
\end{tabular}


Many data points were taken at varying velocities across the range expected in the experiment. A $5^{\text {th }}$ order polynomial curve-fit was used for each calibration. The charts for each calibration are presented in Figure A.1 and Figure A.2. The curve-fit calculated using MS Excel 2007 is also included in the chart along with the $R^{2}$ value. Both curve-fits achieved $R^{2}$ values of 0.99998 which is considerably accurate, although the accuracy can be seen to be poor between zero velocity and the first data point at approximately $40 \mathrm{ft} / \mathrm{s}$. For this reason a $3^{\text {rd }}$ order polynomial curve-fit was used for voltages below that first point (see Figure A.3 and Figure A.4).

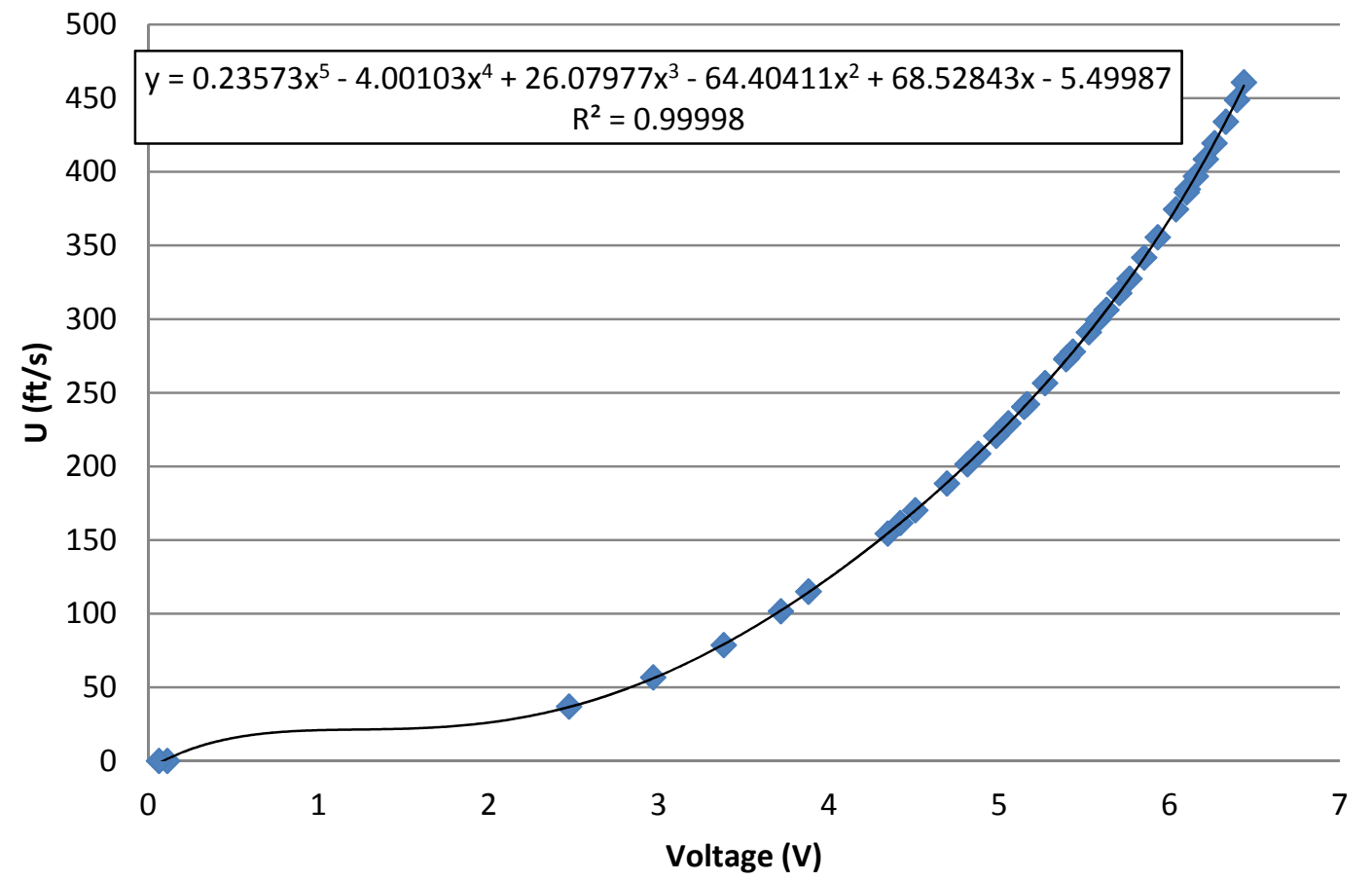

Figure A.1: Calibration data and $5^{\text {th }}$ order polynomial curve-fit for sensor 005005. 


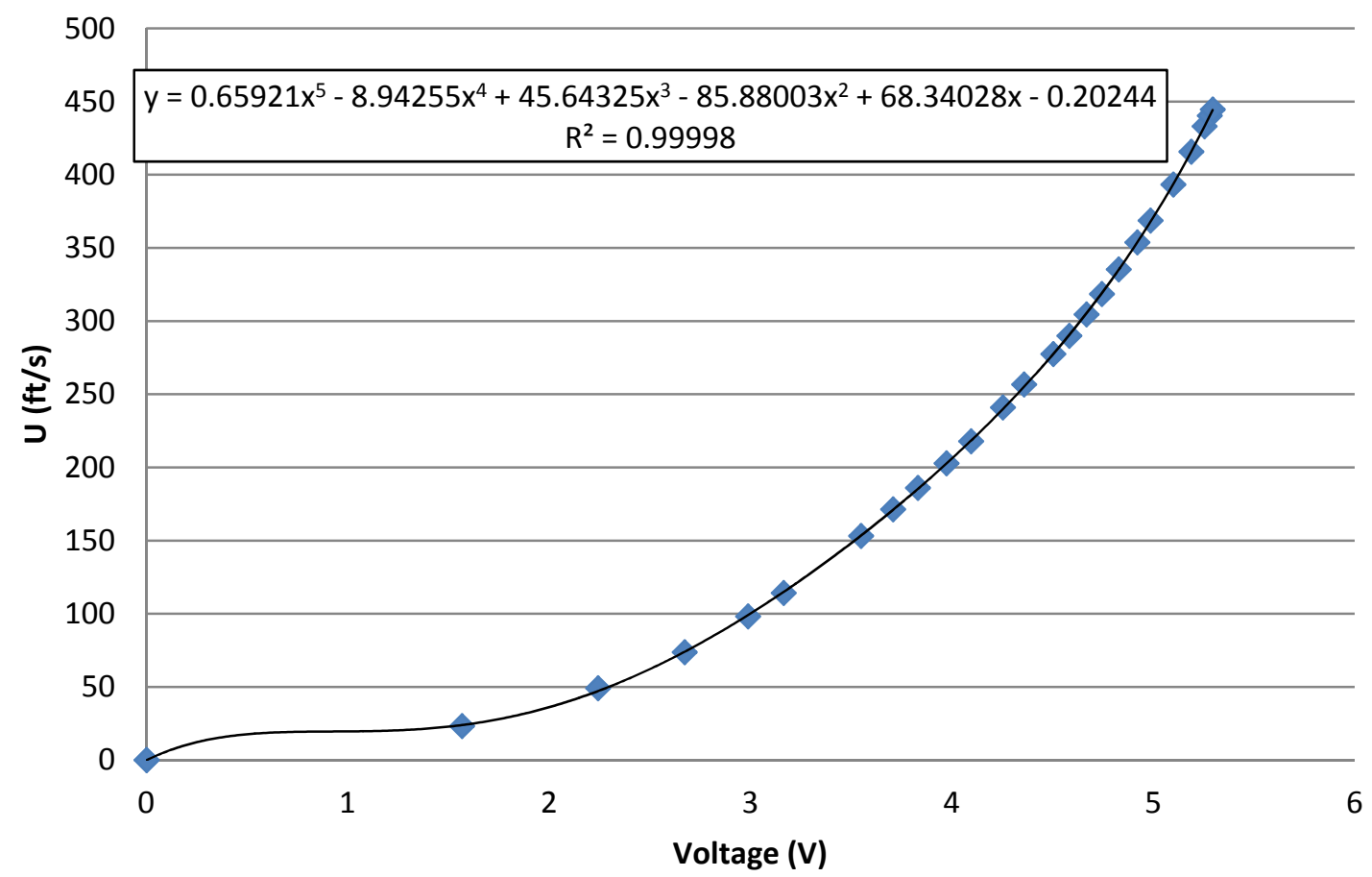

Figure A.2: Calibration data and $5^{\text {th }}$ order polynomial curve-fit for sensor 005006.

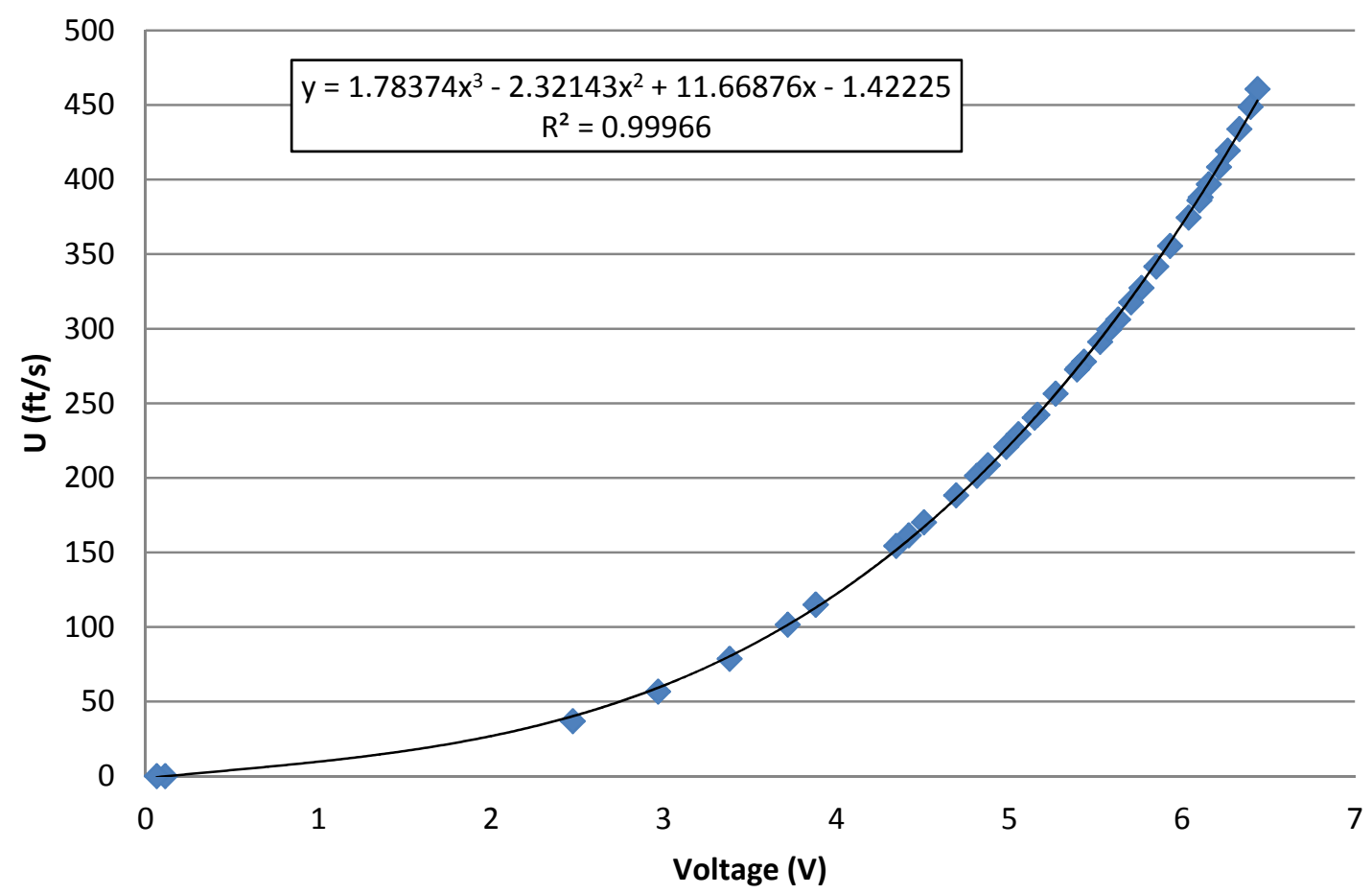

Figure A.3: Calibration data and $3^{\text {rd }}$ order polynomial curve-fit for sensor 005005. 


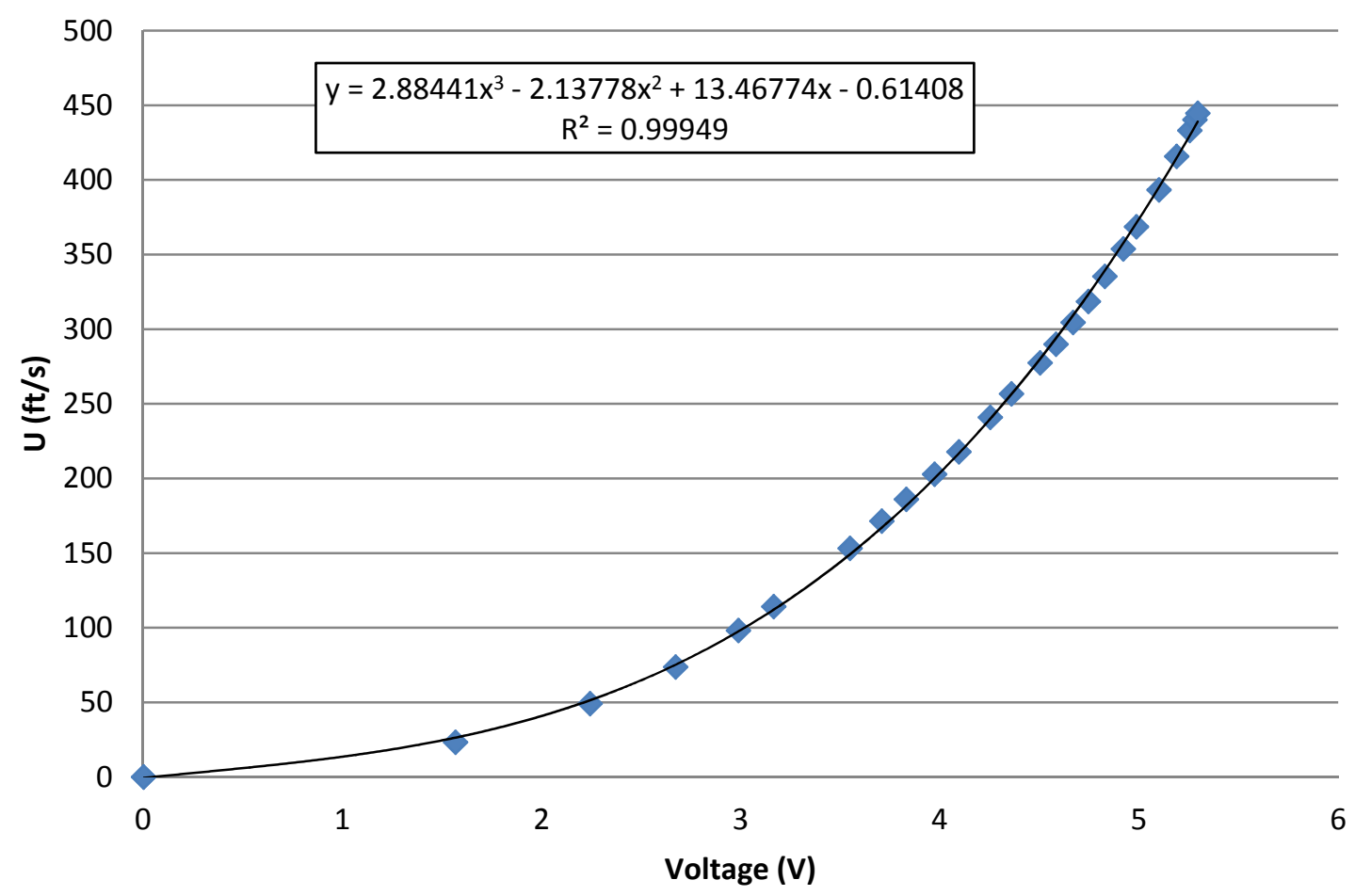

Figure A.4: Calibration data and $3^{\text {rd }}$ order polynomial curve-fit for sensor 005006. 


\section{APPENDIX B. INLET TURBULENCE SCALES AND STATISTICS}

This appendix includes calculated turbulence scales and statistics of the velocity data. The turbulence scales presented in this appendix are the integral length $(\Lambda)$ and time $\left(\tau_{\Lambda}\right)$ scales, the Taylor length $(\lambda)$ and time $\left(\tau_{\lambda}\right)$ microscales, and the Kolmogorov length scale $(\eta)$. The statistics presented include the $1^{\text {st }}, 2^{\text {nd }}, 3^{\text {rd }}$, and $4^{\text {th }}$ order statistical moments, also known as the mean, variance, skewness and kurtosis or flatness, respectively.

It is generally considered that turbulence is composed of eddies of varying sizes. An "eddy" is a turbulent motion that is characterized with length and time scales. The concept of the “energy cascade", first introduced by Richardson [32], suggests that kinetic energy transfers to successively smaller eddies until the eddy size is small enough that its motion stabilizes and molecular viscosity is able to dissipate the kinetic energy [23]. This concept was significantly enhanced by Kolmogorov [33], after whom the smallest scales of eddies is now named.

The integral length scale, $\Lambda$, is a representation of the largest eddies of the flow field whereas the Kolmogorov length scale, $\eta$, is a representation of the smallest. The Taylor microscale, $\lambda$, is a mathematical representation, as opposed to a physical representation, that is determined by the autocorrelation function of the velocity field (see eq. B.2). It is smaller than the integral length scale but larger than the Kolmogorov length scale. The Taylor microscale was incorrectly thought to be the smallest scale of eddies in a turbulent flow field until the Kolmogorov length scale was discovered. 
Each length scale is related to its respective time scale by using Taylor's hypothesis or the frozen-turbulence approximation [34]. This hypothesis approximates that any length scale, $L$, is related to its respective time scale, $\tau$, by the mean velocity of the flow field, $U$ :

$$
U=\frac{L}{\tau}
$$

Methods have been developed to determine the turbulence scales from high frequency velocity data. These methods are found in [23], [25], and [35], and are included here for convenience. The integral time scale, $\tau_{\Lambda}$, can be computed by taking the integral from 0 to $\infty$ of the autocorrelation function between two points in time of the velocity data, $R(s)$, which is defined:

$$
R(s)=\frac{\overline{\mathrm{u}(\mathrm{t}) \mathrm{u}(\mathrm{t}+\mathrm{s})}}{\overline{\mathrm{u}^{2}(\mathrm{t})}}
$$

In general, it is considered an acceptable approximation to integrate $R(s)$ to the first zero crossing to obtain $\tau_{\Lambda}$. Using Taylor's hypothesis and $\tau_{\Lambda}$, the integral length scale, $\Lambda$, can be calculated. Assuming Taylor's hypothesis again, stationary probe velocity data can be used to determine the autocorrelation function, $f(r)$, between two points in space of the flow field. The Taylor microscale, $\lambda$, can be computed by defining a parabola, $p(r)$, where $p(0)=f(0), p^{\prime}(0)=f^{\prime}(0)$, and $p^{\prime \prime}(0)=f^{\prime \prime}(0)$. The Taylor microscale is determined by $\lambda=r_{l}$ where $p\left(r_{l}\right)=0$. The Taylor timescale can be determined with the Taylor microscale using Taylor's hypothesis. The Kolmogorov length scale, $\eta$, is a function of the dissipation rate, $\varepsilon$, and the kinematic viscosity of air, $v$ :

$$
\eta=\frac{v^{\frac{3}{4}}}{\varepsilon^{\frac{1}{4}}}
$$


where $\varepsilon$ can be estimated with the RMS of the fluctuating velocity, $u_{r m s}$ (defined in eq. 5.1 ), and the integral length scale, $\Lambda$ :

$$
\varepsilon=\frac{u_{r m s}{ }^{3}}{\Lambda}
$$

Since turbulence is very difficult to predict or characterize analytically, statistical analyses are the best current method available to characterize the behavior of turbulent flow. The statistical moments are defined in Table B.1 for convenience. The data is represented by the letter $u$, and $\mathrm{N}$ is the number of data points of the data sample. The variance is the square of the standard deviation, which is usually denoted $\sigma$. Hence variance is denoted as the square of the standard deviation, $\sigma^{2}$. The standard deviation of velocity data is the same as the RMS of the fluctuating velocity $u_{r m s}$ (defined in eq. 5.1) in this study. The third and fourth order moments are typically normalized by the standard deviation (raised to the third and fourth power, respectively) to obtain the skewness and the kurtosis, respectively.

Table B.1: Definitions of statistical moments for discrete sets of data.

\begin{tabular}{lcc}
\hline Moment & Equation & Eq. No. \\
\hline Mean $-U$ & $\frac{\sum u}{N}$ & (B.5) \\
Variance $-\sigma^{2}$ & $\frac{\sum(u-U)^{2}}{N}$ \\
Skewness $-\gamma_{1}$ & $\frac{\sum(u-U)^{3} / N}{\sigma^{3}}$ \\
Kurtosis $-\gamma_{2}$ & $\frac{\sum(u-U)^{4} / N}{\sigma^{4}}$ \\
\hline
\end{tabular}


The calculated turbulence scales for select data samples from this study are presented in Table B.2. Though not a consistent trend, the flows with higher levels of turbulence generally have smaller length scales than the baseline flow. This is likely due to the turbulence screens which cut up the length scales larger than their openings as the flow passes through. The Kolmogorov length scale is consistently lower in the turbulence cases. This indicates that using a grid to increase the turbulence of a flow also decreases the scale at which viscous forces completely dissipate turbulent kinetic energy.

The calculated statistical moments for select data samples from this study are presented in Table B.3. The mean values are similar across the test configurations. The standard deviation values again demonstrate the elevated level of turbulence for the turbulence configurations. The elevated turbulence configurations exhibit a sharp drop in magnitude of skewness compared to the baseline. This is expected because turbulent flows generally mix well producing a normal distribution of velocity. It is observed that the magnitude of the flatness is slightly less in the elevated inlet freestream turbulence cases than in the baseline case. 
Table B.2: Calculated turbulence scales of velocity data at select locations for each turbulence configuration

\begin{tabular}{|c|c|c|c|c|c|c|c|}
\hline Scale & $\theta$ & $y / H$ & $\mathbf{z} / \mathbf{W}$ & Baseline & High Screen & Single Screen & Dual Screens \\
\hline \multirow{6}{*}{$\Lambda(\mathrm{ft})$} & \multirow{3}{*}{0} & 0 & \multirow{3}{*}{ - } & 2.5283 & 0.014746 & 0.86654 & 0.015681 \\
\hline & & 0.56 & & 0.85445 & 3.2686 & 0.077602 & 0.071701 \\
\hline & & 0.98 & & 0.89550 & 1.0394 & 0.10812 & 0.075035 \\
\hline & \multirow{3}{*}{90} & \multirow{3}{*}{ - } & 0 & 0.0075770 & 0.15256 & 0.0074010 & 0.11570 \\
\hline & & & 0.47 & 0.79098 & 0.25475 & 0.077543 & 0.069360 \\
\hline & & & 1.00 & 2.9128 & 0.60568 & 0.61673 & 0.49733 \\
\hline \multirow{6}{*}{$\begin{array}{c}\tau_{\Lambda} \\
(\mu \mathrm{s})\end{array}$} & \multirow{3}{*}{0} & $\overline{0}$ & \multirow{3}{*}{ - } & 7613.0 & 25.000 & 2560.0 & 44.000 \\
\hline & & 0.56 & & 3022.0 & 10514 & 267.00 & 260.00 \\
\hline & & 0.98 & & 3935.0 & 4040.0 & 429.00 & 337.000 \\
\hline & \multirow{3}{*}{90} & & 0 & 16.000 & 337.00 & 16.000 & 285.00 \\
\hline & & - & 0.47 & 2272.0 & 875.00 & 234.00 & 240.00 \\
\hline & & & 1.00 & 12199 & 2991.0 & 2601.0 & 2509 \\
\hline \multirow{6}{*}{$\lambda(\mathrm{ft})$} & \multirow{3}{*}{0} & $\overline{0}$ & \multirow{3}{*}{ - } & 0.010462 & 0.014746 & 0.023257 & 0.0096950 \\
\hline & & 0.56 & & 0.016206 & 0.035860 & 0.026267 & 0.025609 \\
\hline & & 0.98 & & 0.022123 & 0.034303 & 0.026032 & 0.021866 \\
\hline & \multirow{3}{*}{90} & & 0 & 0.012566 & 0.013879 & 0.12329 & 0.016621 \\
\hline & & - & 0.47 & 0.011827 & 0.010201 & 0.015124 & 0.010733 \\
\hline & & & 1.00 & 0.042741 & 0.024594 & 0.029251 & 0.026065 \\
\hline \multirow{6}{*}{$\begin{array}{c}\tau_{\lambda} \\
(\mu \mathrm{s})\end{array}$} & \multirow{3}{*}{0} & 0 & & 32.000 & 25.000 & 69.000 & 27.000 \\
\hline & & 0.56 & - & 57.000 & 115.00 & 90.000 & 93.000 \\
\hline & & 0.98 & & 97.000 & 133.00 & 103.000 & 98.000 \\
\hline & \multirow{3}{*}{90} & & 0 & 27.000 & 31.000 & 27.000 & 41.000 \\
\hline & & - & 0.47 & 34.000 & 35.000 & 46.000 & 37.000 \\
\hline & & & 1.00 & 179.00 & 121.00 & 123.00 & 132.00 \\
\hline \multirow{6}{*}{$\eta(\mathrm{ft})$} & \multirow{3}{*}{0} & 0 & & 0.000216 & 0.000157 & 0.000212 & 0.000138 \\
\hline & & 0.56 & - & 0.000330 & 0.000288 & 0.000217 & 0.000243 \\
\hline & & 0.98 & & 0.000314 & 0.000317 & 0.000223 & 0.000198 \\
\hline & \multirow{3}{*}{90} & & 0 & 0.000815 & 0.000200 & 0.000176 & 0.000168 \\
\hline & & - & 0.47 & 0.000267 & 0.000172 & 0.000185 & 0.000141 \\
\hline & & & 1.00 & 0.000243 & 0.000279 & 0.000209 & 0.000132 \\
\hline
\end{tabular}


Table B.3: Calculated statistical moments of velocity data at select locations

\begin{tabular}{|c|c|c|c|c|c|c|c|}
\hline Moment & $\theta$ & $\mathbf{y} / \mathbf{H}$ & $\mathbf{z} / \mathbf{W}$ & Baseline & High Screen & Single Screen & Dual Screens \\
\hline \multirow{6}{*}{$\begin{array}{c}1^{\text {st }} \\
\text { Mean } \\
(\mathrm{ft} / \mathrm{s})\end{array}$} & \multirow{3}{*}{0} & 0 & \multirow{3}{*}{ - } & 332.09 & 417.96 & 338.43 & 354.86 \\
\hline & & 0.56 & & 282.76 & 310.88 & 290.78 & 275.37 \\
\hline & & 0.98 & & 227.59 & 257.23 & 251.91 & 222.63 \\
\hline & \multirow{3}{*}{90} & \multirow{3}{*}{ - } & 0 & 461.62 & 452.97 & 454.23 & 406.61 \\
\hline & & & 0.47 & 348.16 & 291.14 & 331.45 & 289.27 \\
\hline & & & 1.00 & 238.78 & 202.49 & 237.11 & 198.21 \\
\hline \multirow{6}{*}{$\begin{array}{c}2^{\text {nd }} \\
\text { Std. Dev. } \\
\text { (ft/s) }\end{array}$} & \multirow{3}{*}{0} & 0 & \multirow{3}{*}{ - } & 3.3574 & 6.4191 & 7.7591 & 7.6089 \\
\hline & & 0.56 & & 2.2365 & 6.4821 & 8.3787 & 6.5156 \\
\hline & & 0.98 & & 3.3594 & 5.1126 & 7.8565 & 8.3370 \\
\hline & \multirow{3}{*}{90} & \multirow{3}{*}{ - } & 0 & 0.28364 & 5.2083 & 5.9880 & 8.8537 \\
\hline & & & 0.47 & 2.4905 & 5.1821 & 6.6590 & 8.0672 \\
\hline & & & 1.00 & 10.890 & 4.7445 & 10.006 & 8.0264 \\
\hline \multirow{6}{*}{$\begin{array}{c}3^{\text {rd }} \\
\text { Skewness }\end{array}$} & \multirow{3}{*}{0} & 0 & \multirow{3}{*}{ - } & 0.27598 & 0.11889 & -0.040614 & 0.03391 \\
\hline & & 0.56 & & -0.38684 & -0.18399 & -0.074003 & 0.028280 \\
\hline & & 0.98 & & -0.89546 & -0.28162 & 0.060478 & 0.012473 \\
\hline & \multirow{3}{*}{90} & & 0 & -38.092 & -1.7592 & -1.9248 & 0.062411 \\
\hline & & - & 0.47 & -0.11537 & 0.19391 & 0.001376 & -0.026000 \\
\hline & & & 1.00 & 0.63217 & 0.83373 & 0.42092 & 0.39144 \\
\hline \multirow{6}{*}{$\begin{array}{c}4^{\text {th }} \\
\text { Kurtosis }\end{array}$} & \multirow{3}{*}{0} & 0 & \multirow{3}{*}{ - } & 3.3492 & 3.2361 & 2.9307 & 2.9633 \\
\hline & & 0.56 & & 3.2753 & 2.5614 & 2.9009 & 2.9880 \\
\hline & & 0.98 & & 3.792706 & 3.2542 & 3.0538 & 2.7559 \\
\hline & \multirow{3}{*}{90} & & 0 & 2036.6 & 7.9942 & 8.1378 & 3.6520 \\
\hline & & - & 0.47 & 3.0874 & 2.9752 & 3.0250 & 2.9209 \\
\hline & & & 1.00 & 3.7508 & 6.7158 & 8.8827 & 3.3501 \\
\hline
\end{tabular}




\section{APPENDIX C. AXIAL STATIC PRESSURE DATA AT OFF-DESIGN THROAT MACH NUMBERS}

This appendix contains axial static pressure data from inside the diffuser on the upper surface at varying throat Mach numbers. Axial static pressure data were taken at off-design throat Mach numbers ranging from 0.550 to 0.725 . There are seven total data points (in addition to what was already presented in Chapter 5) for the single screen and baseline test scenarios. No off-design data points were taken with the either the dual screens or high screen test configurations.

The data at each throat Mach number for the two test configurations are presented in Figure C.1 and Figure C.2. Additionally, the data with each test configuration is compared at each individual off-design throat Mach number. A scaled in chart is included for each comparison to examine the separation layer region. These charts are presented in Figures C.3 C.16.

It can be seen that changing the throat Mach number has the effect of off-setting the axial static pressure profile, but the trend is maintained. The difference between the single screen configuration and the baseline configuration is maintained across the range of off-design throat Mach numbers as well. This observation is accentuated in the scaled in comparisons of the static pressure profiles in the separation region. 


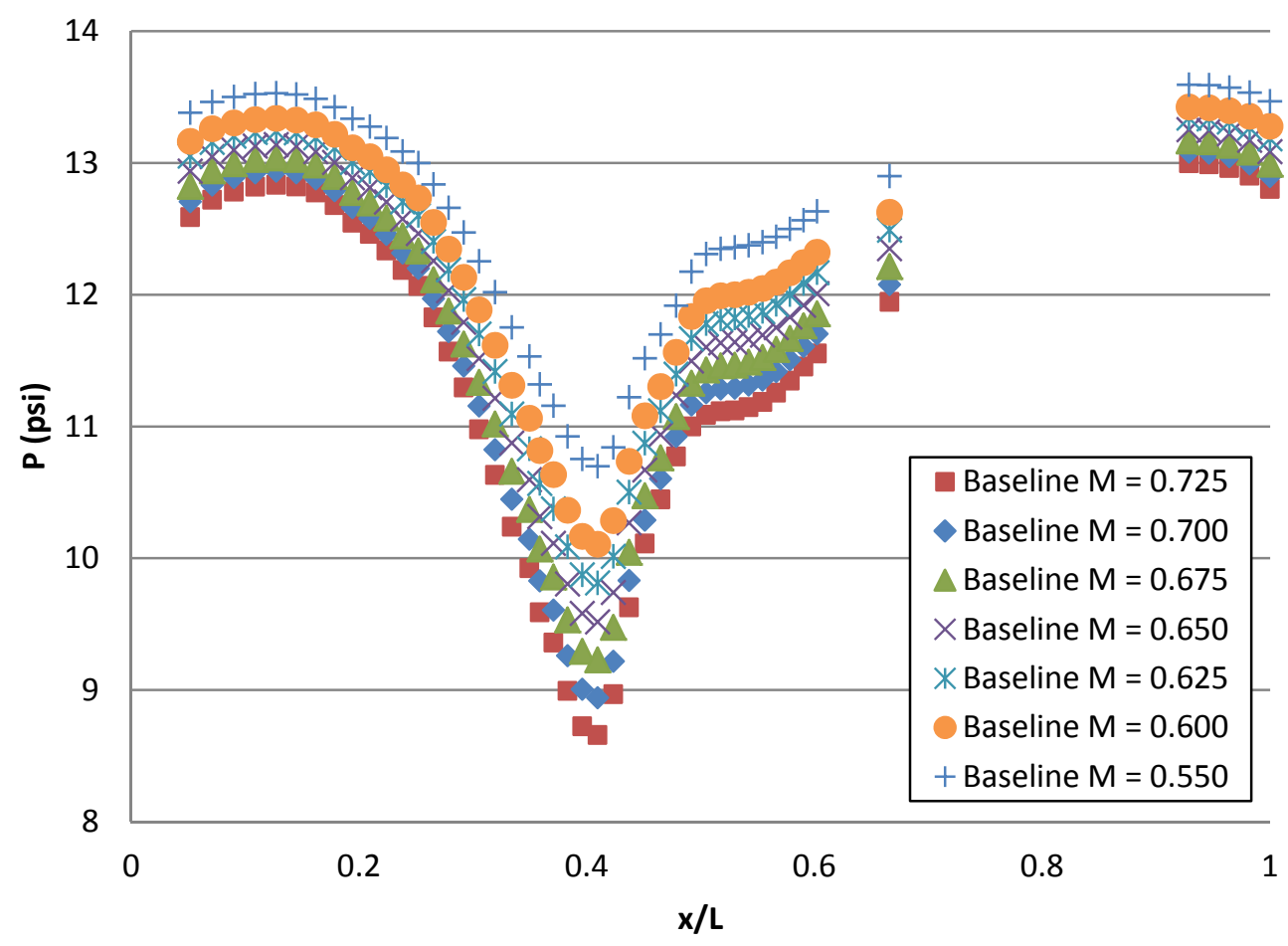

Figure C.1: Axial static pressures along upper surface of diffuser with varying Mach numbers for the baseline configuration.

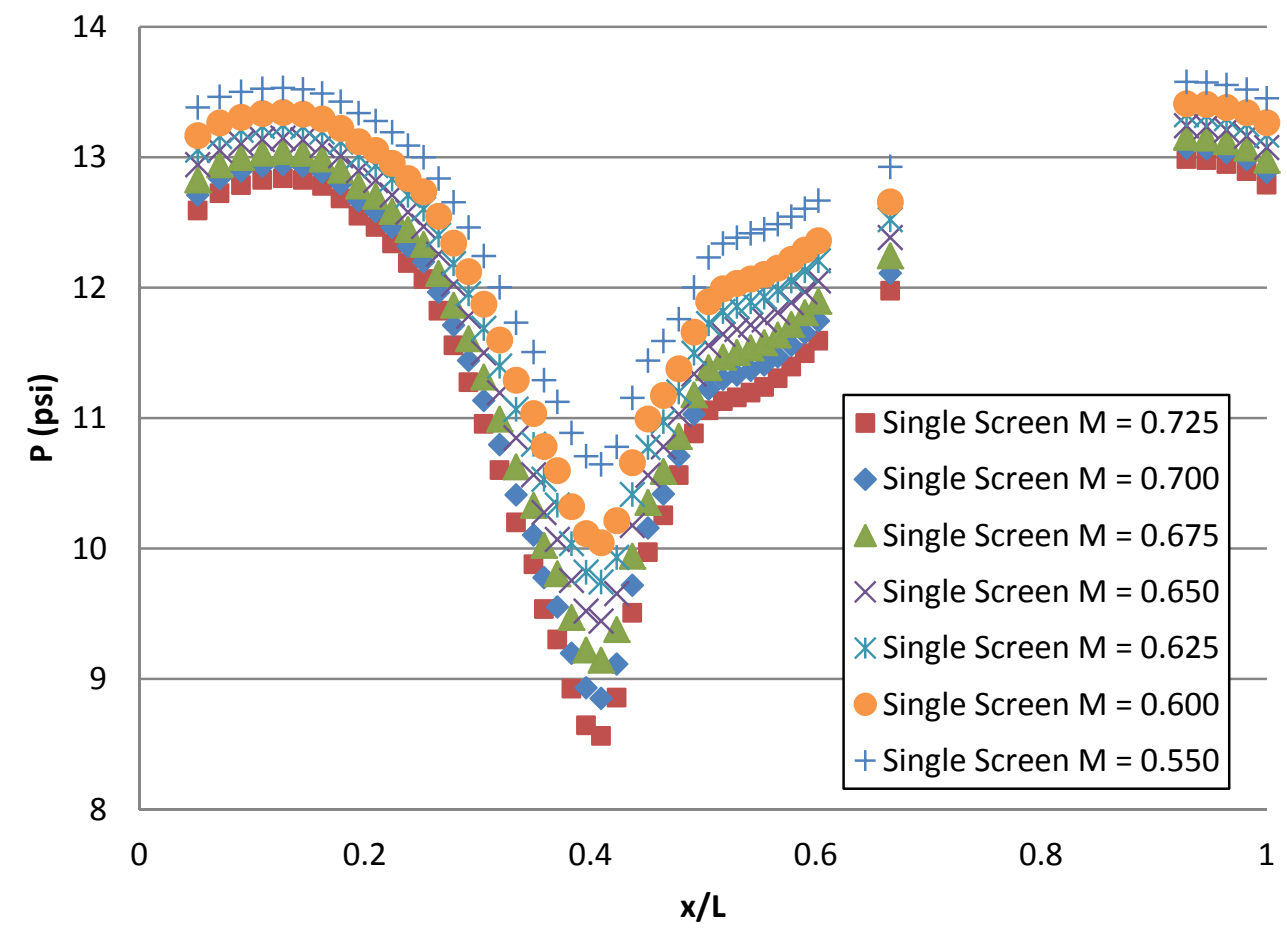

Figure C.2: Axial static pressures along upper surface of diffuser with varying Mach numbers for the single screen configuration. 


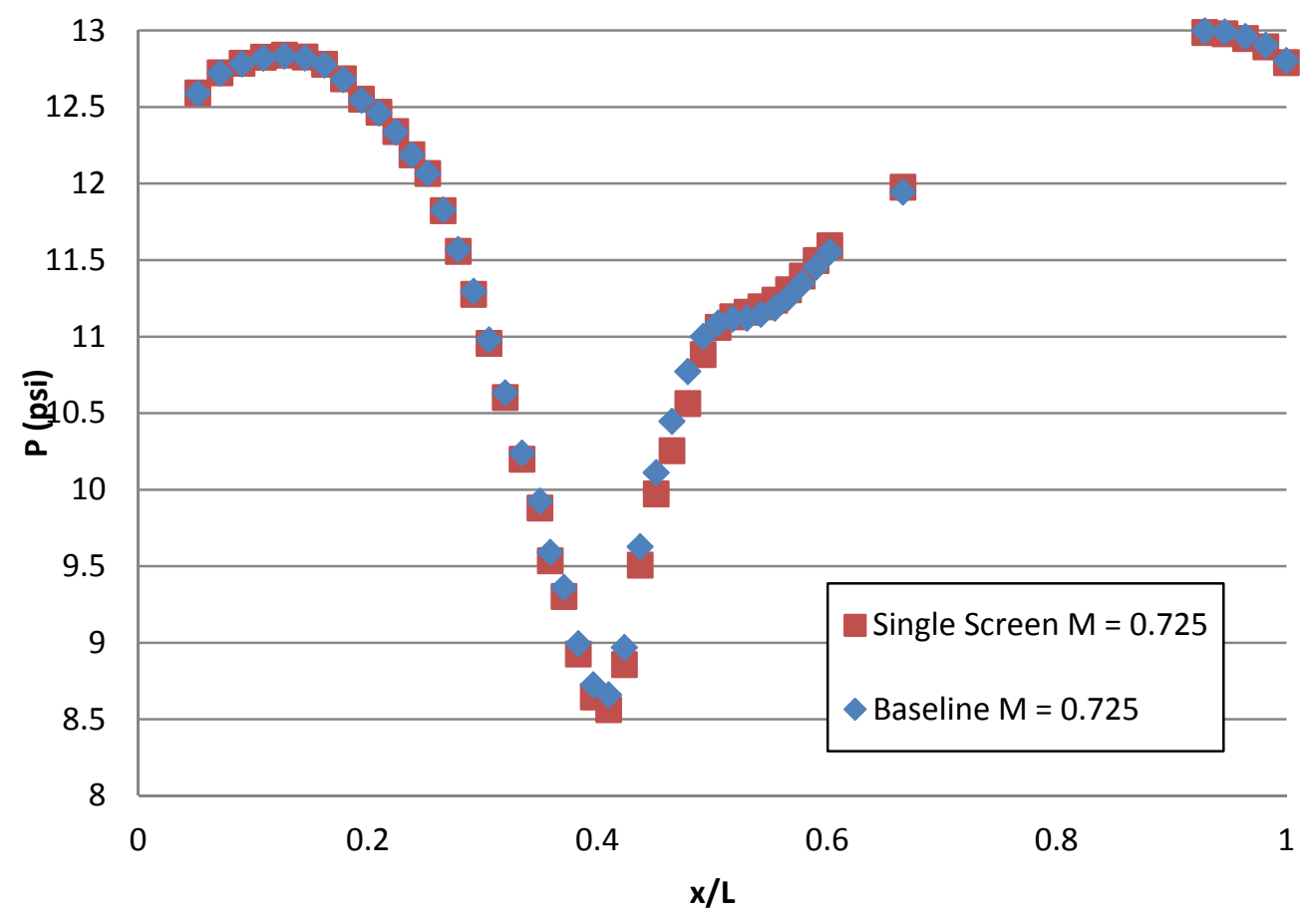

Figure C.3: Comparison of upper axial static pressures at $\mathrm{M}=0.725$.

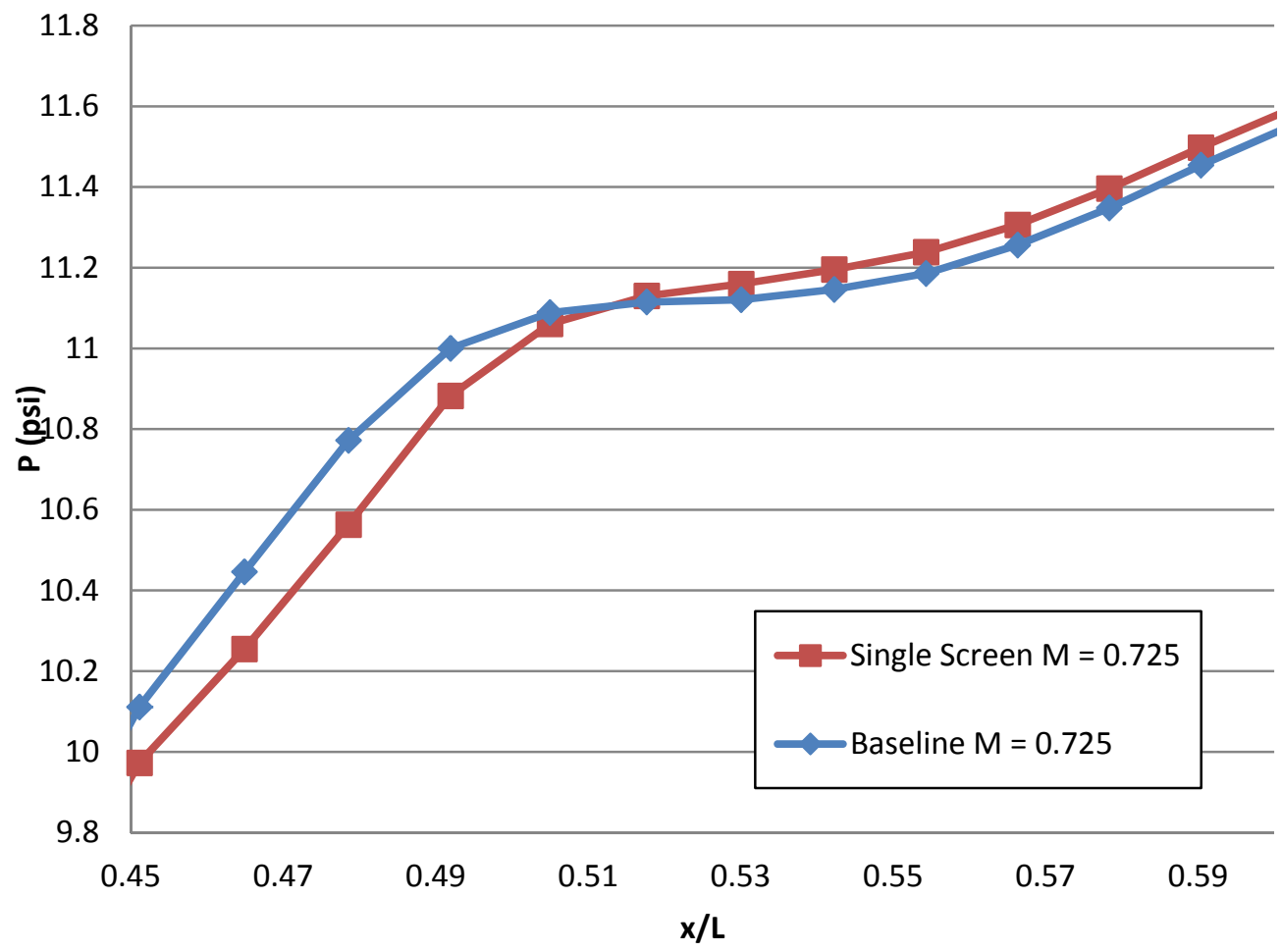

Figure C.4: Scaled in comparison of upper axial static pressures at $\mathrm{M}=0.725$. 


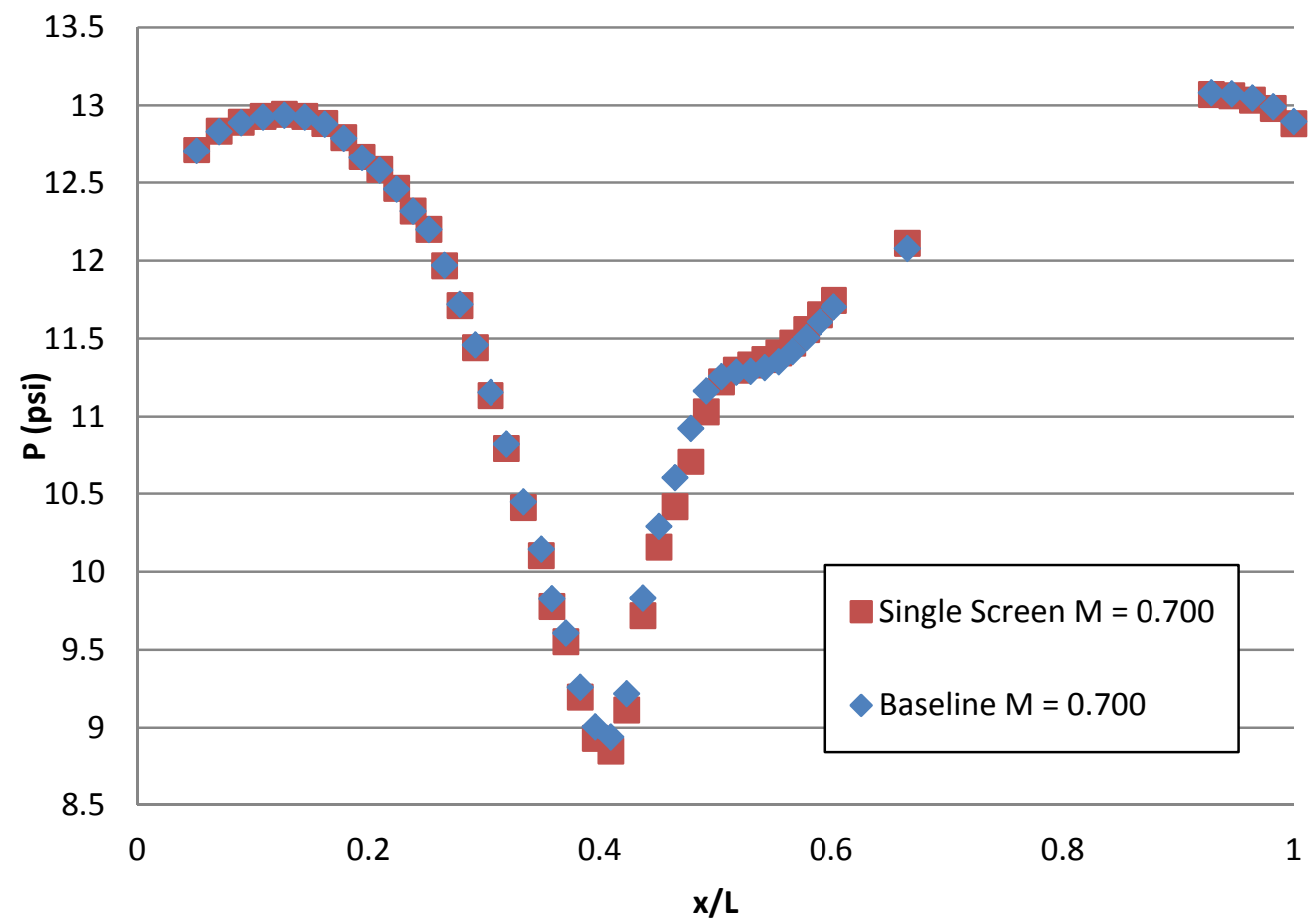

Figure C.5: Comparison of upper axial static pressures at $\mathrm{M}=0.700$.

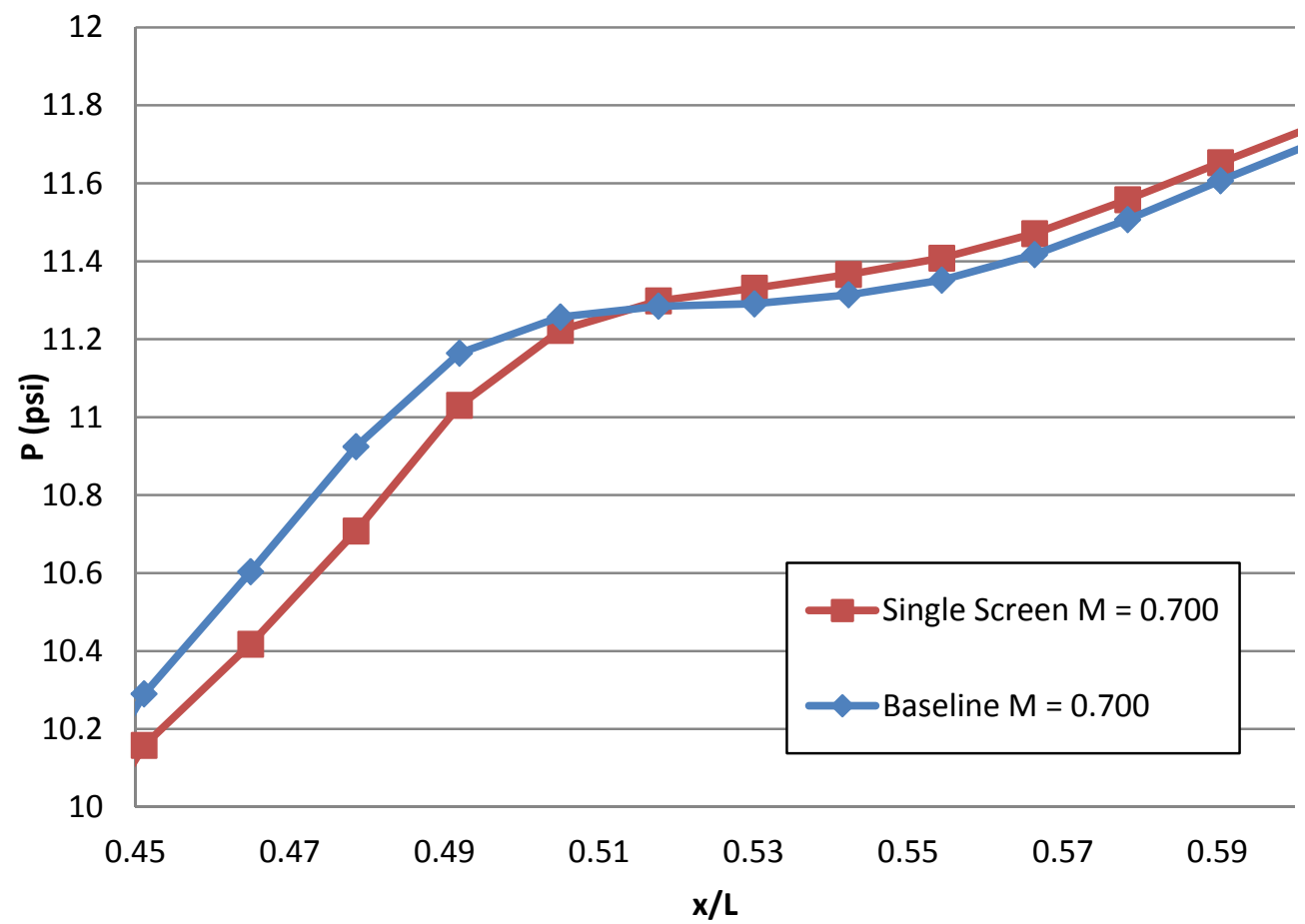

Figure C.6: Scaled in comparison of upper axial static pressures at $\mathrm{M}=0.700$. 


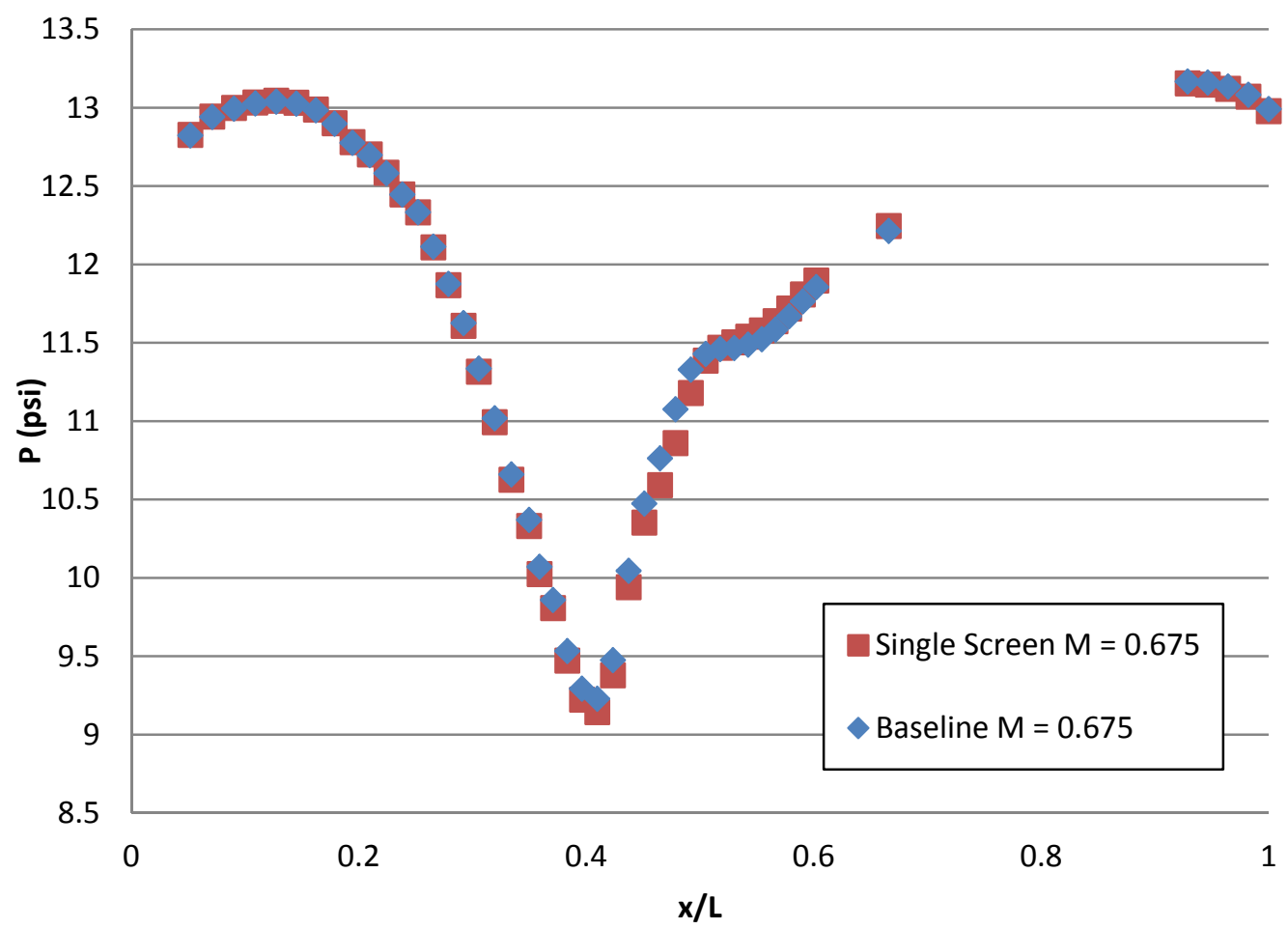

Figure C.7: Comparison of upper axial static pressures at $\mathrm{M}=0.675$.

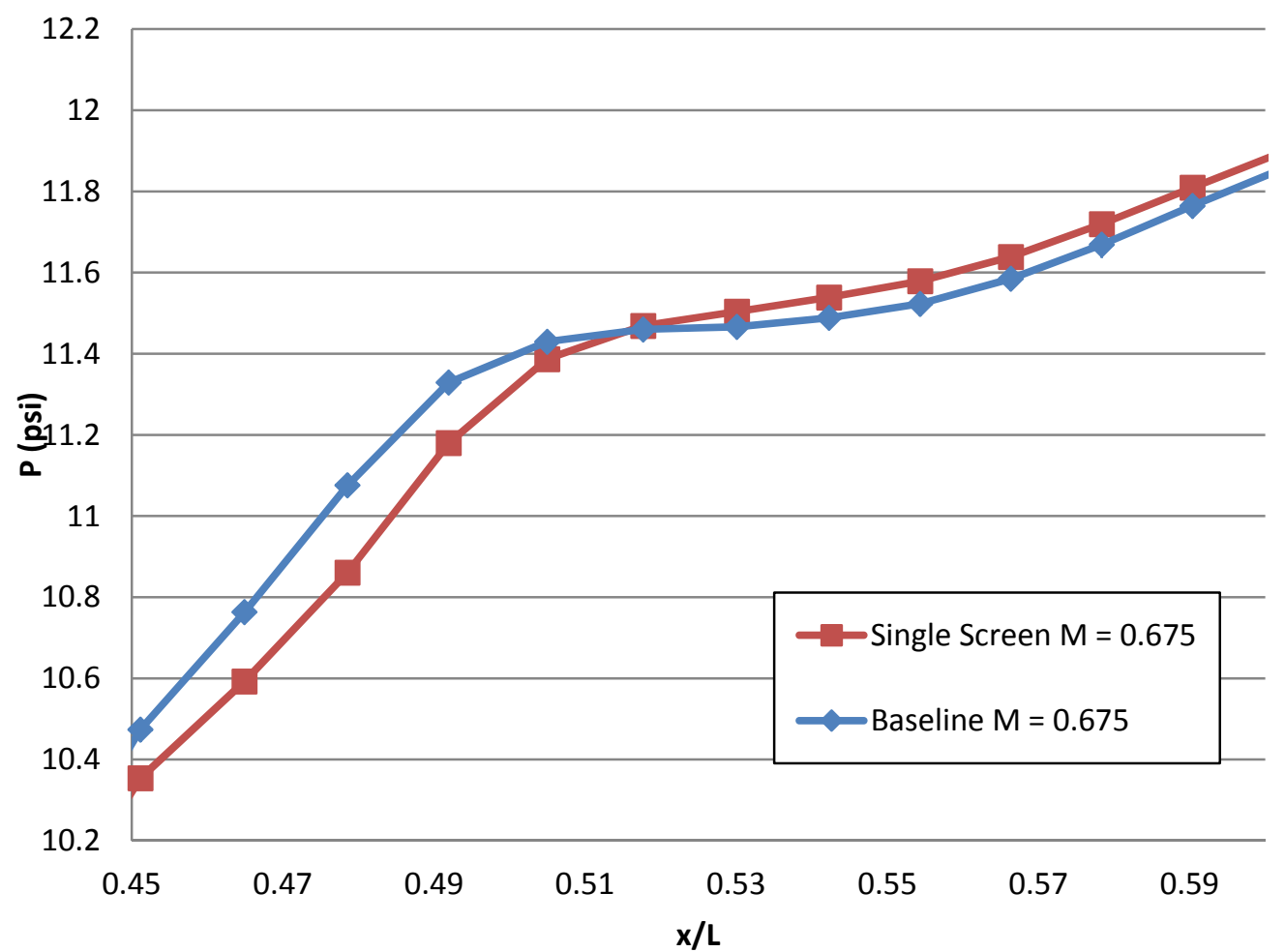

Figure C.8: Scaled in comparison of upper axial static pressures at $\mathrm{M}=0.675$. 


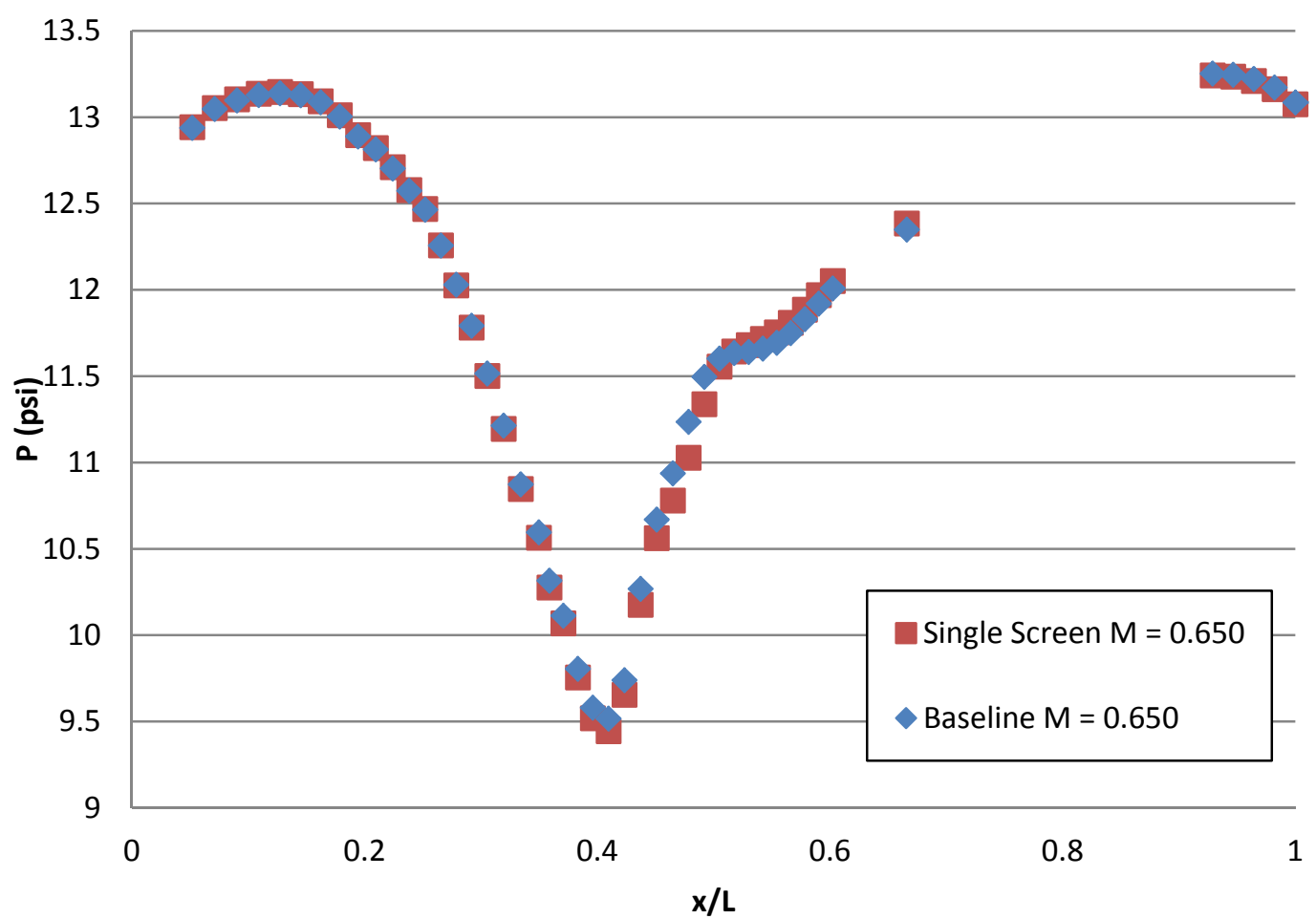

Figure C.9: Comparison of upper axial static pressures at $\mathrm{M}=0.650$.

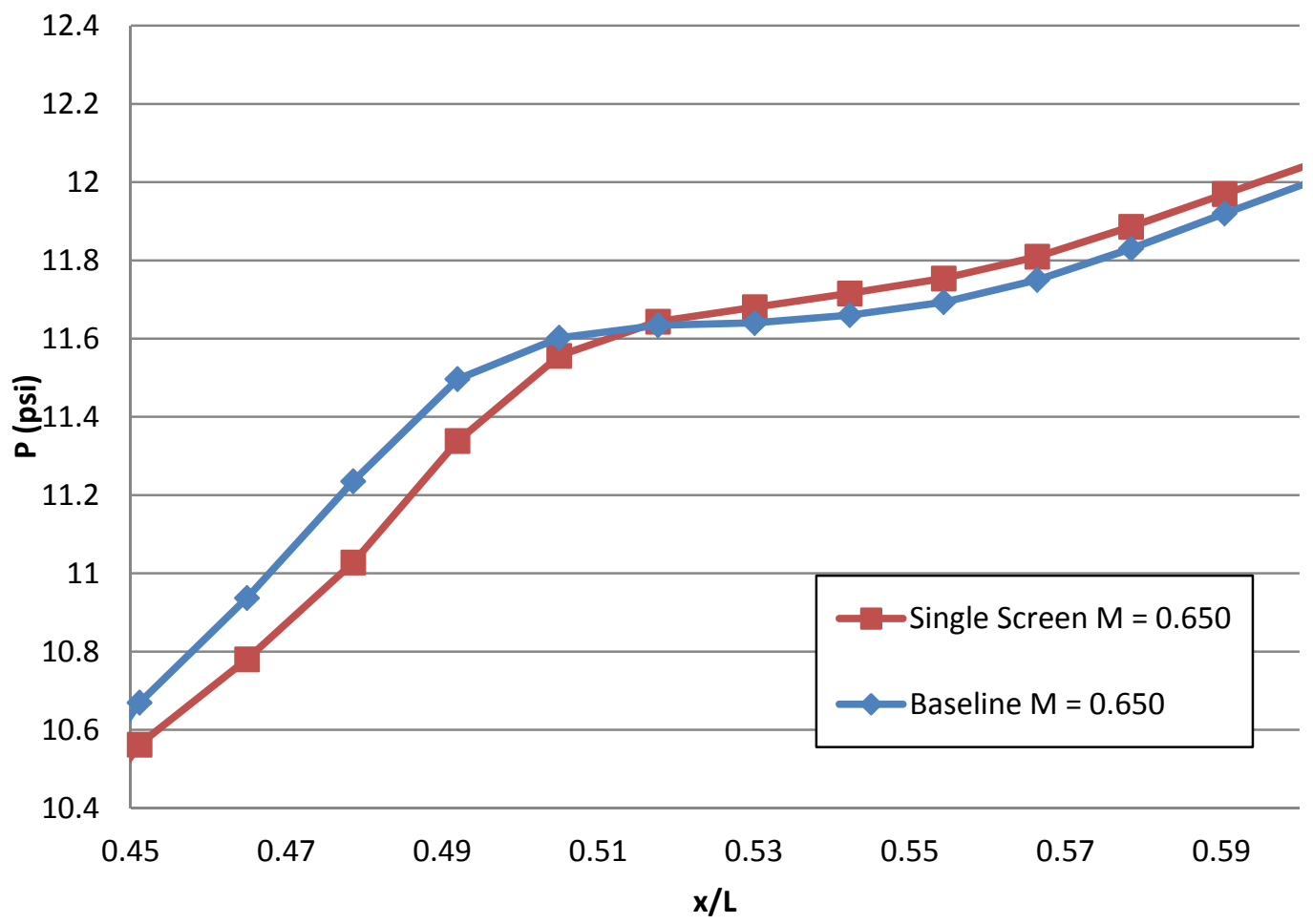

Figure C.10: Scaled in comparison of upper axial static pressures at $\mathrm{M}=0.650$. 


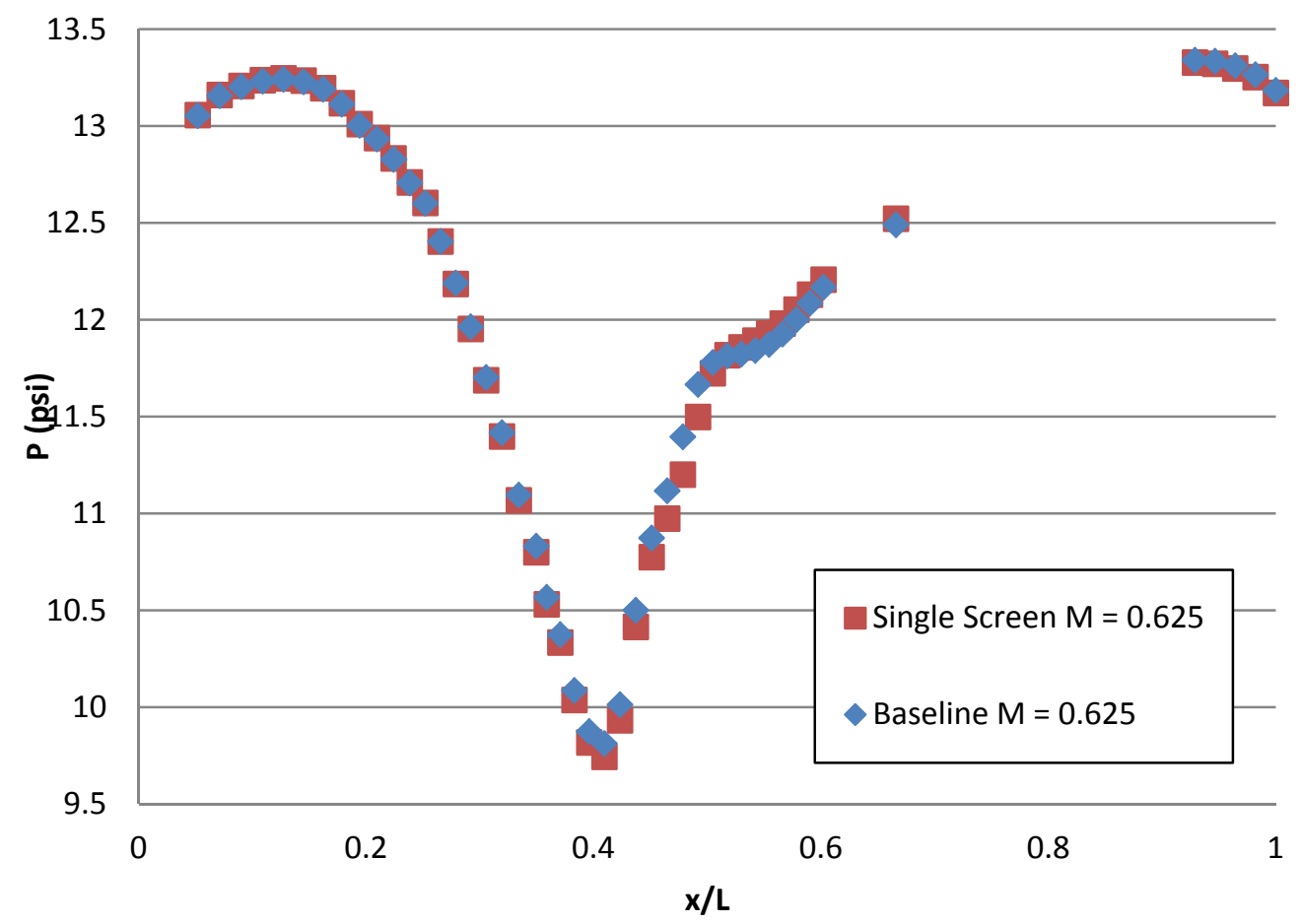

Figure C.11: Comparison of upper axial static pressures at $\mathrm{M}=0.625$.

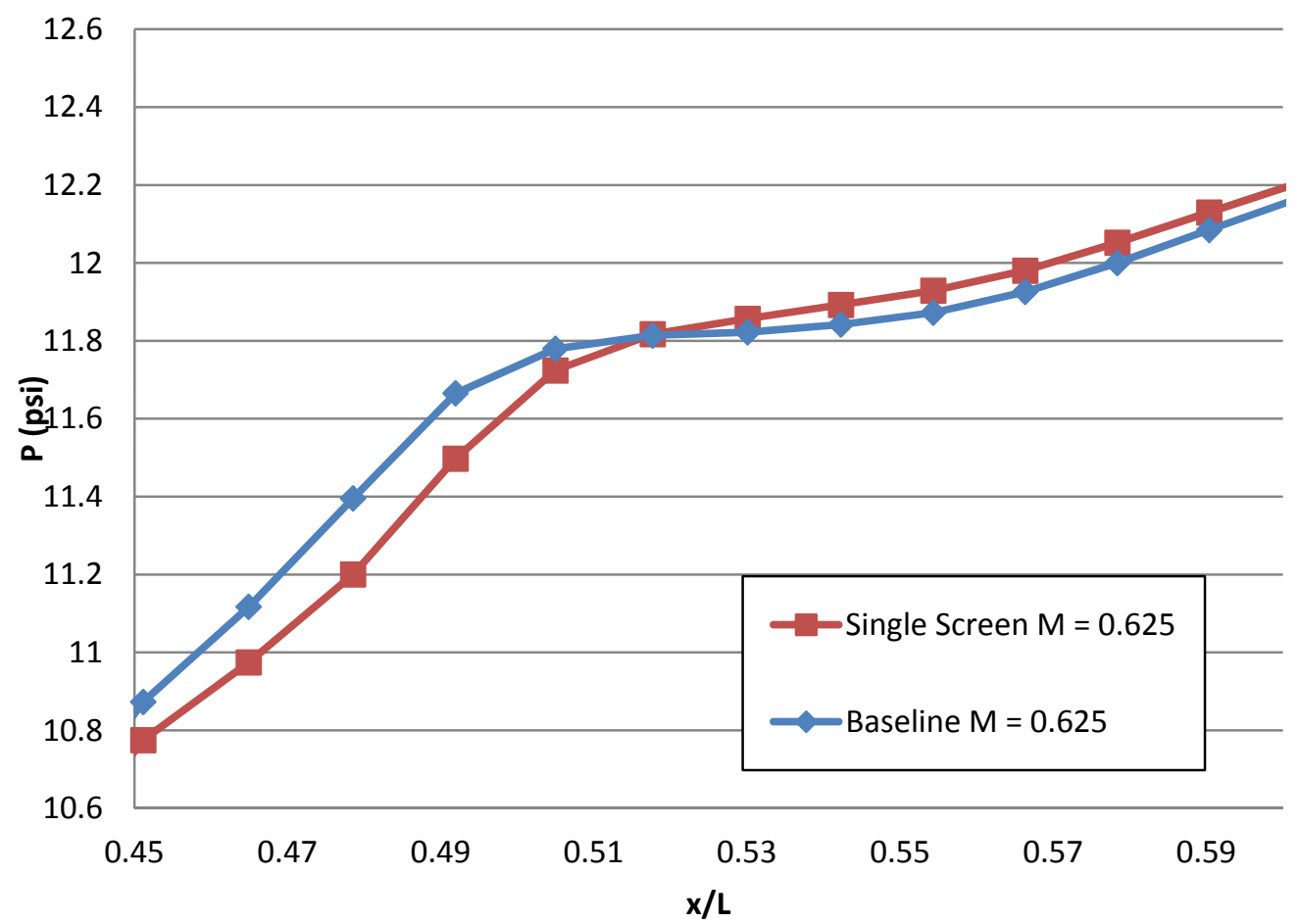

Figure C.12: Scaled in comparison of upper axial static pressures at $\mathrm{M}=0.625$. 


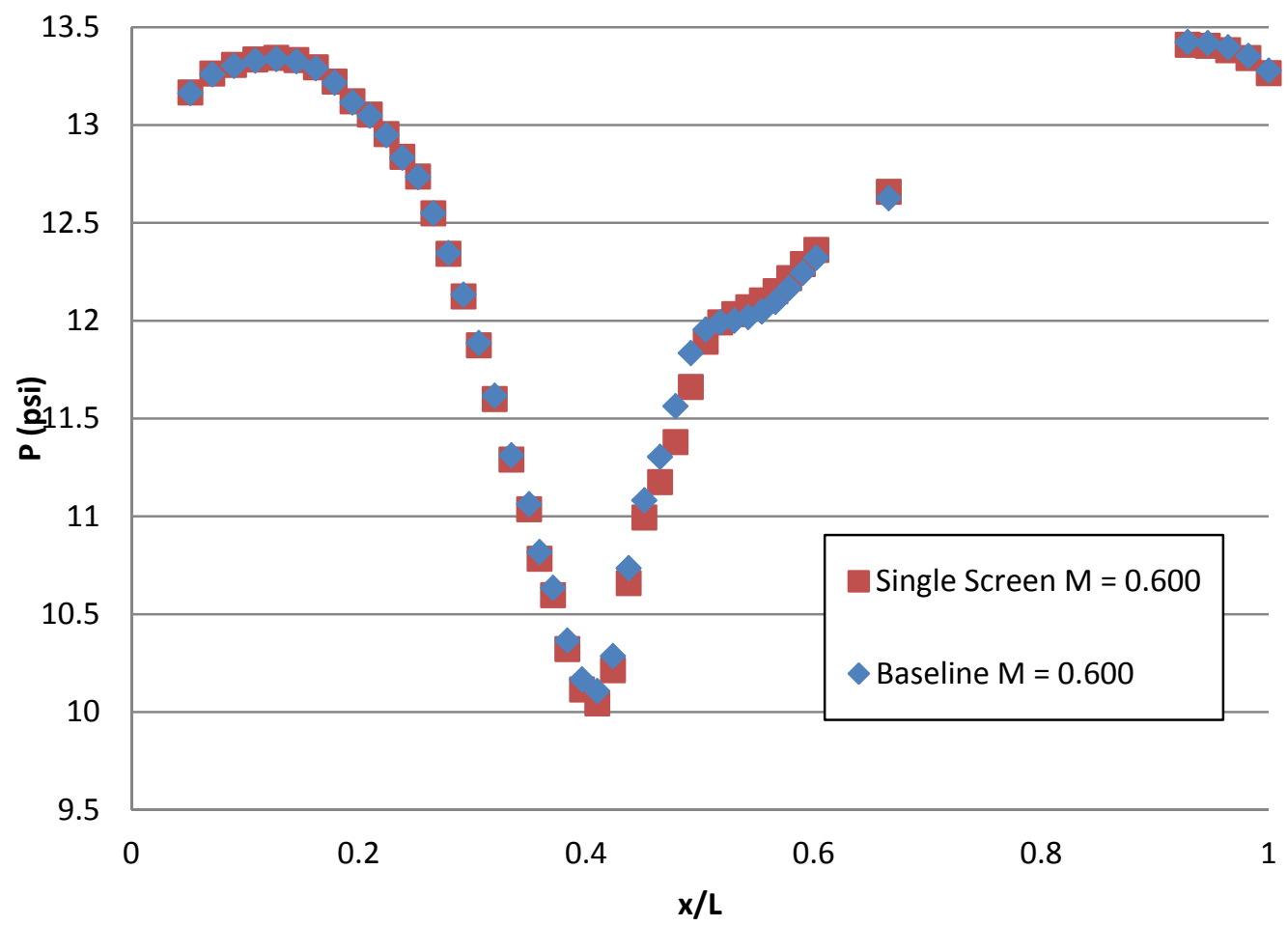

Figure C.13: Comparison of upper axial static pressures at $\mathrm{M}=0.600$.

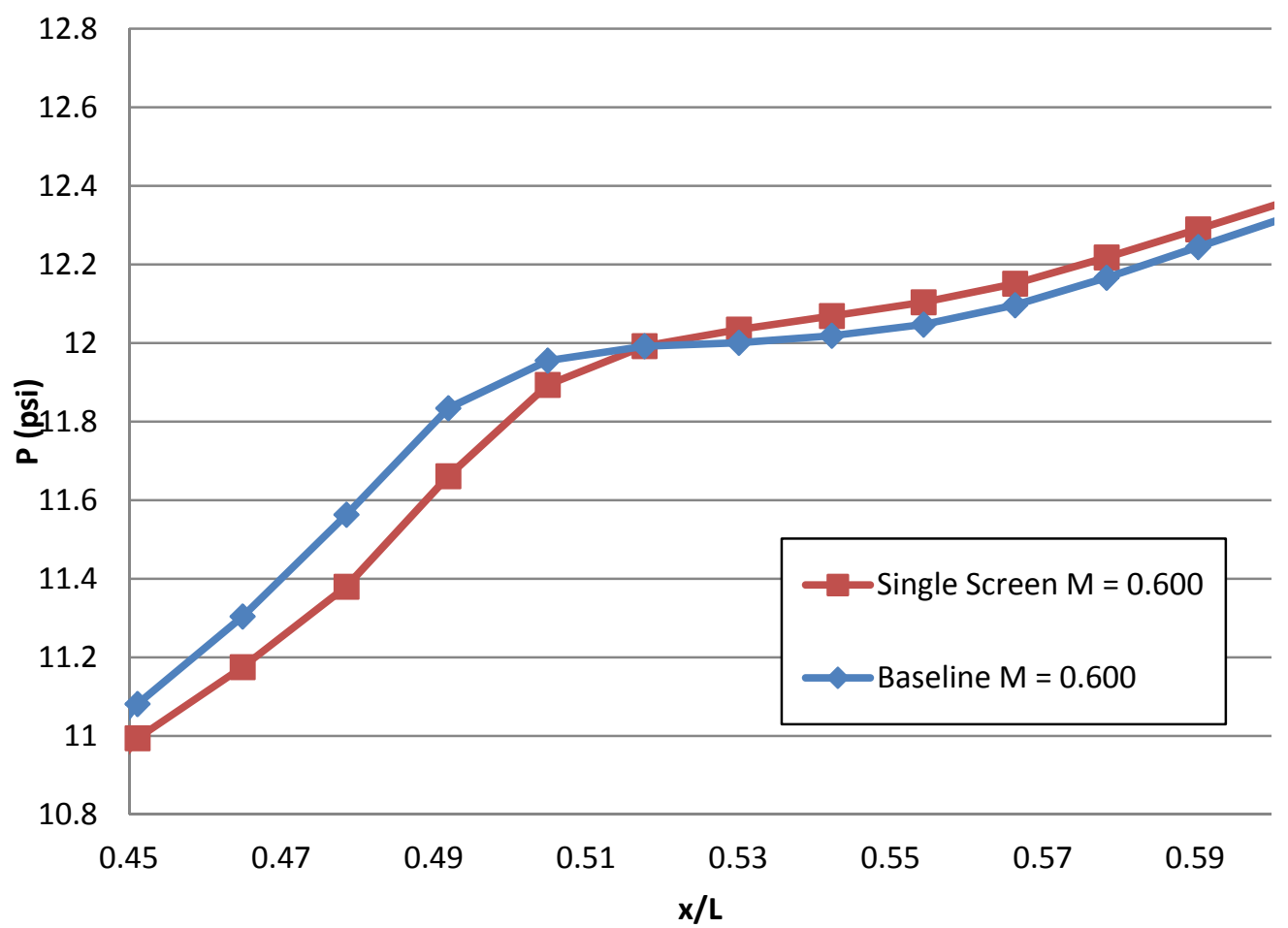

Figure C.14: Scaled in comparison of upper axial static pressures at $\mathrm{M}=0.600$. 


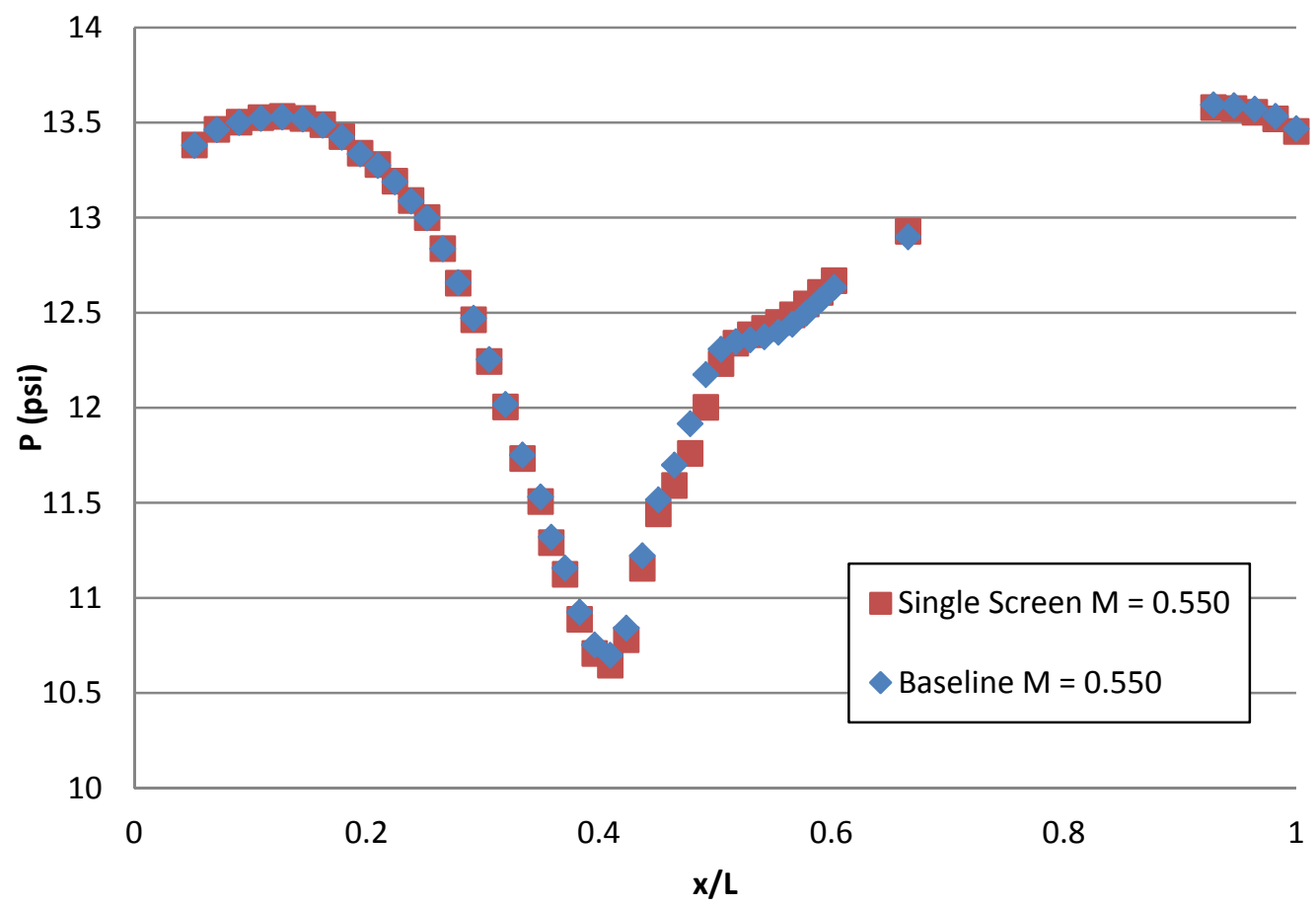

Figure C.15: Comparison of upper axial static pressures at $\mathrm{M}=0.550$.

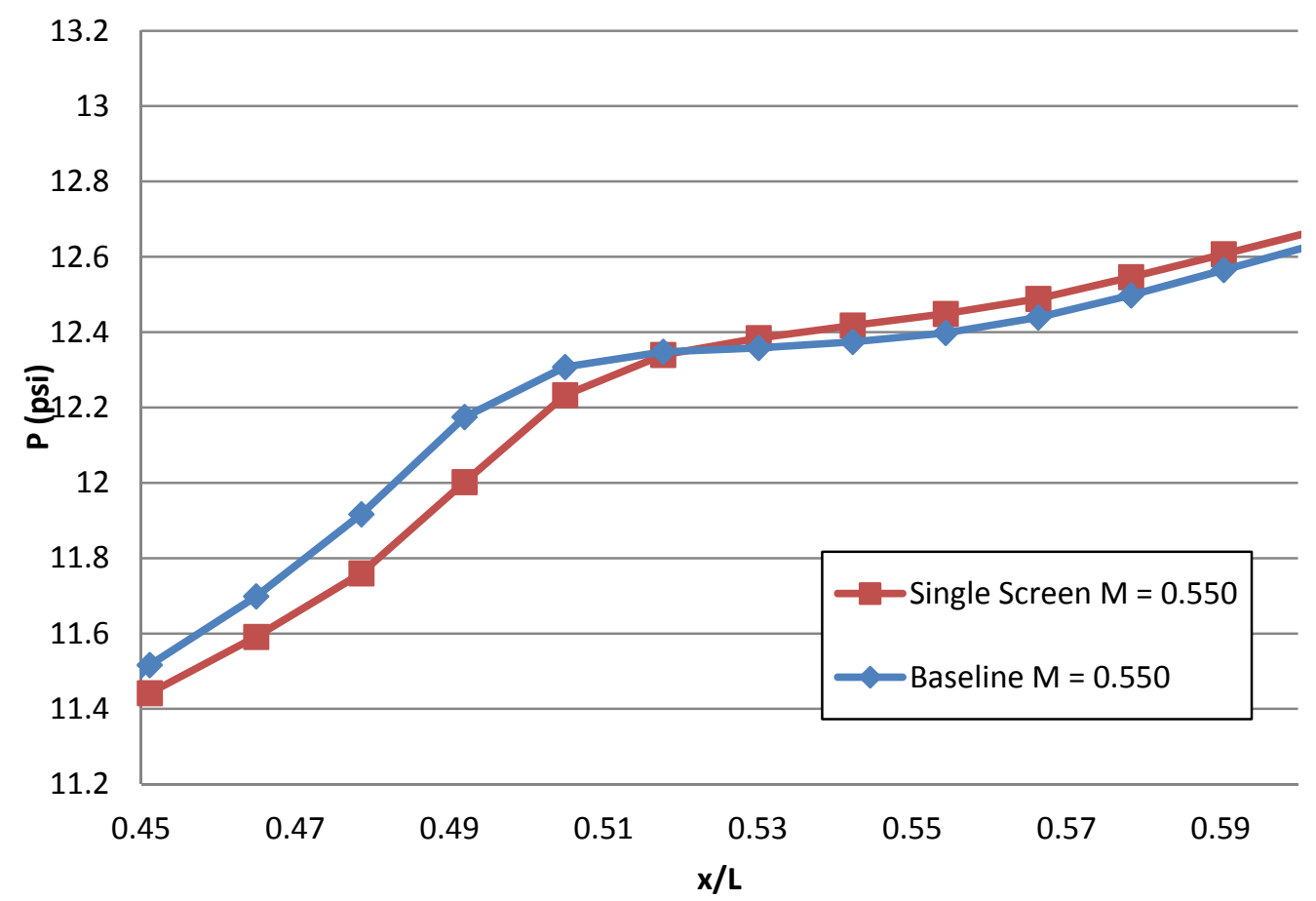

Figure C.16: Scaled in comparison of upper axial static pressures at $\mathrm{M}=0.550$. 


\section{APPENDIX D. RE-SCALED AIP TOTAL PRESSURE DATA PLOTS}

This appendix contains some additional AIP total pressure contours that could be useful in visualizing the difference between the turbulence cases and the baseline. The contours presented in this appendix include the original differential contours that were presented in Chapter 5 for reference, but include re-scaled contours that show just the negative and just the positive changes due to each turbulence configuration. The contours are presented in Figures D.1 - D.9.

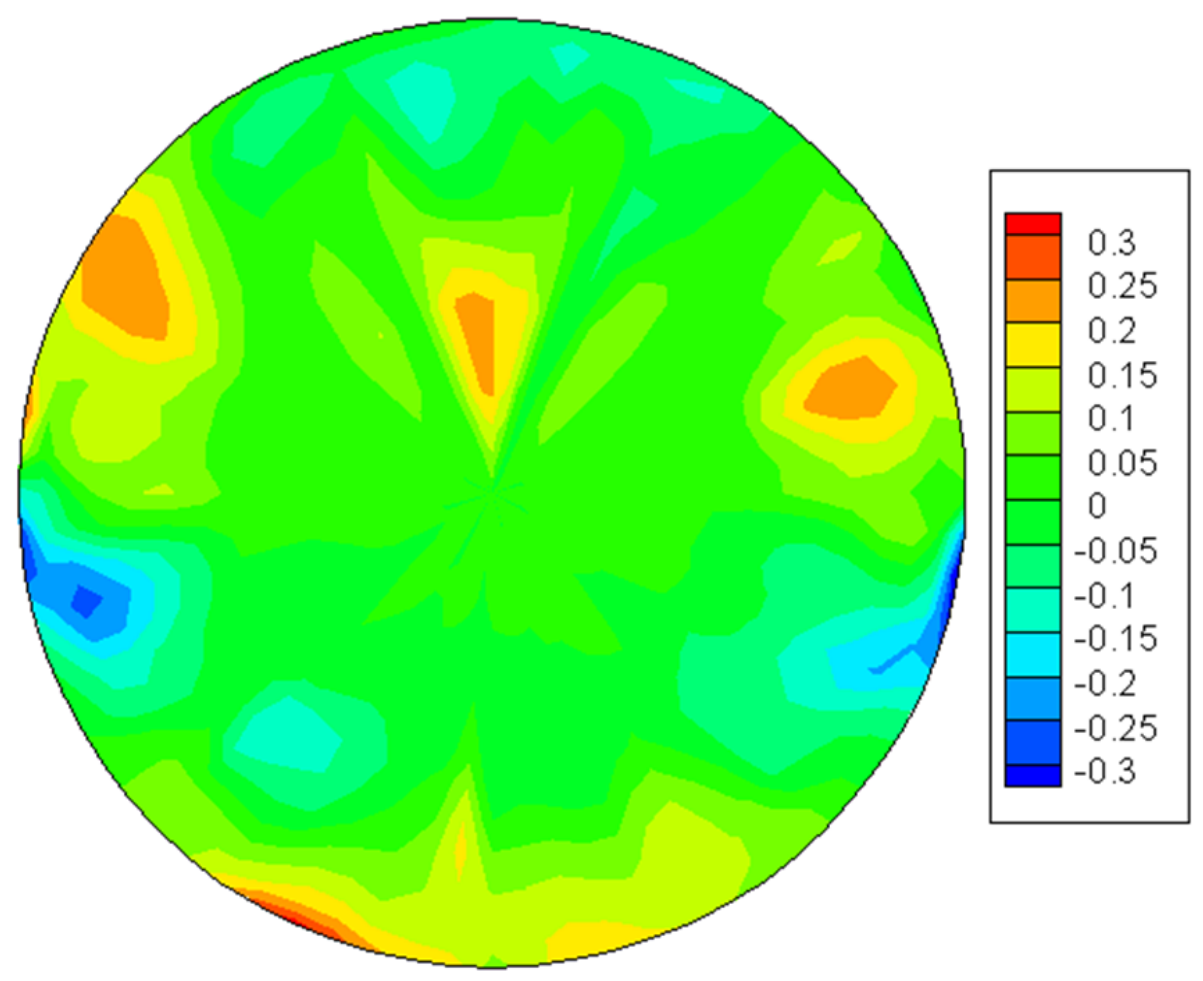

Figure D.1: Total pressure difference between single screen and baseline (psi). 


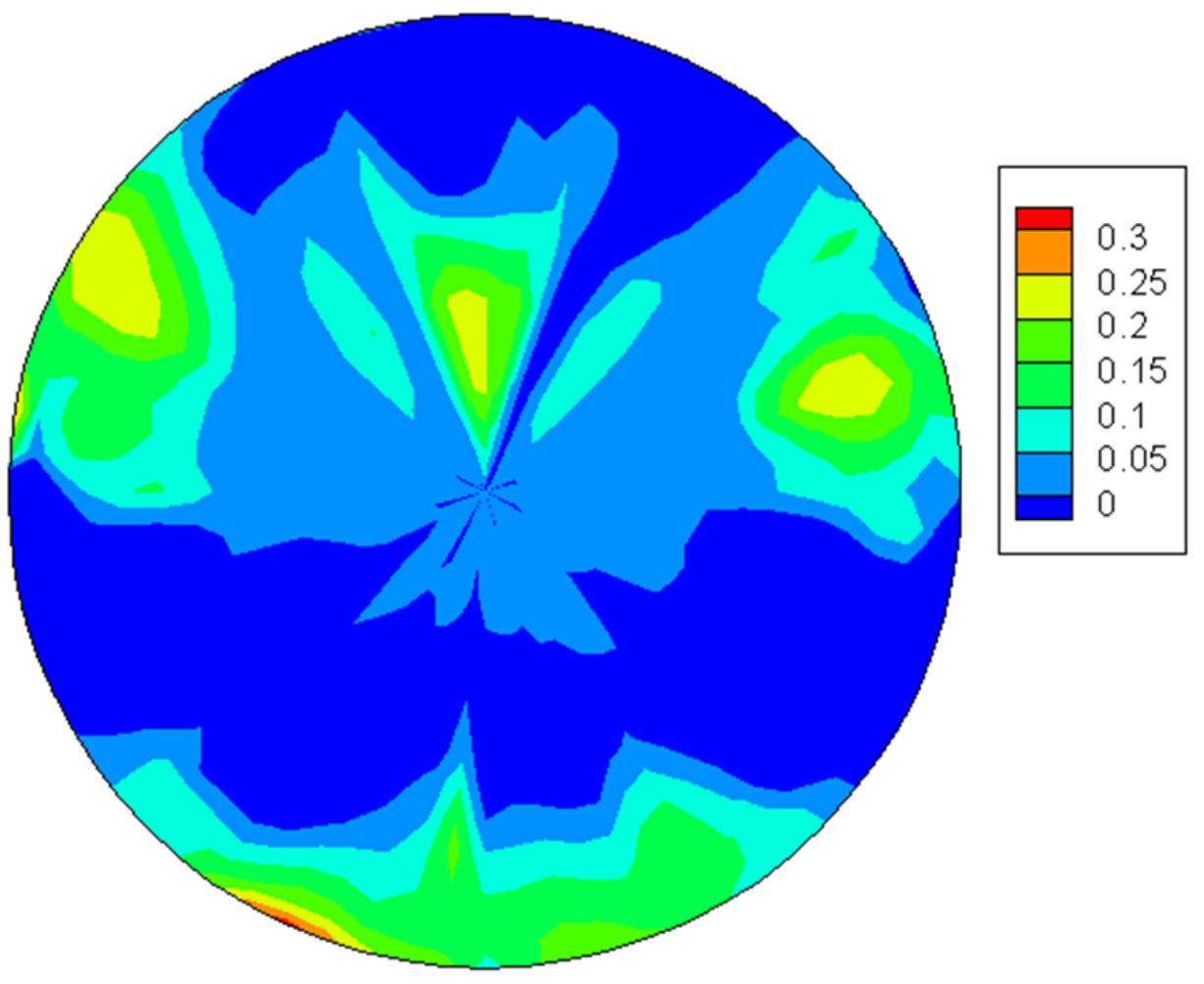

Figure D.2: Positive total pressure difference between single screen and baseline (psi).

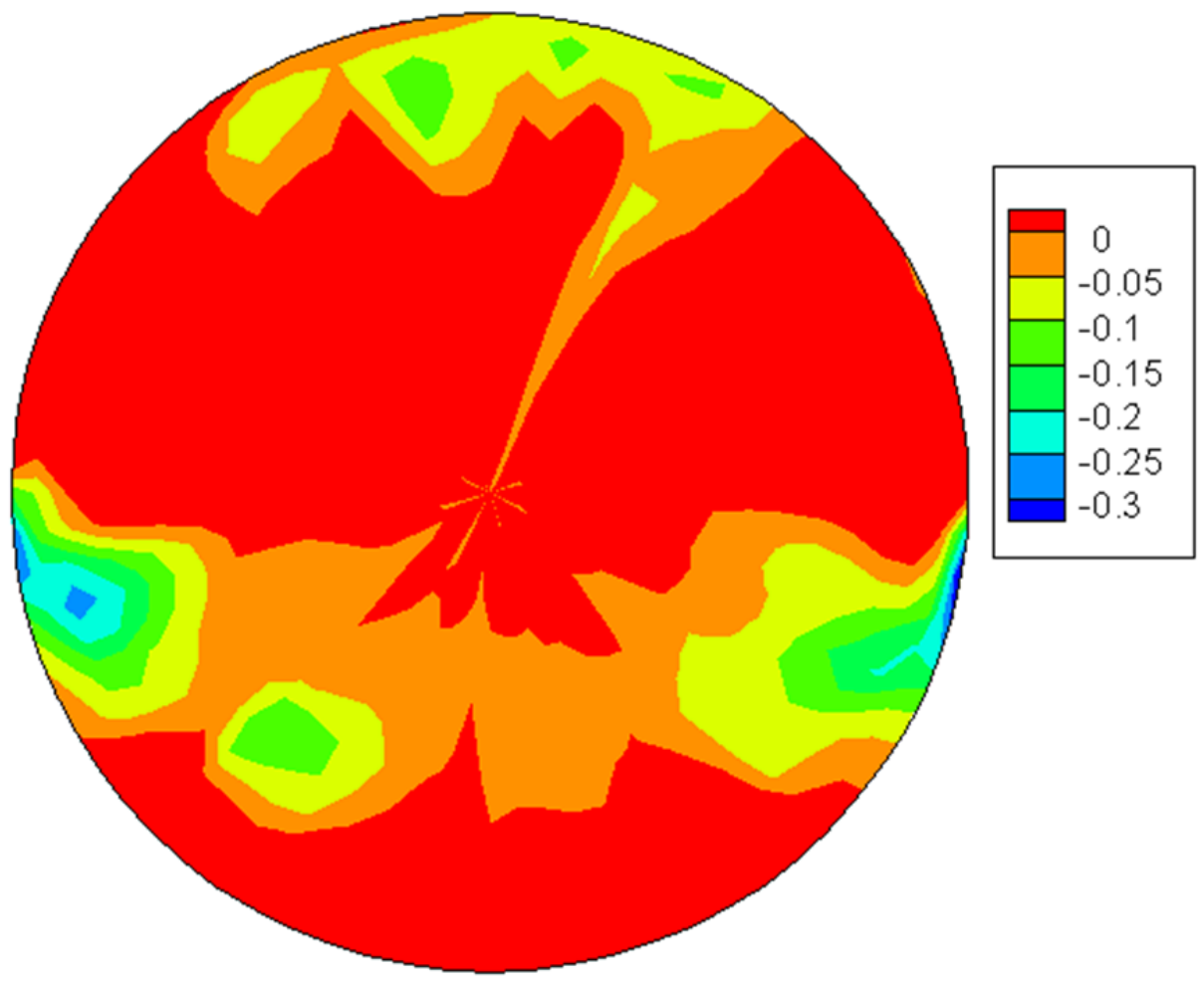

Figure D.3: Negative total pressure difference between Single Screen and Baseline (psi). 


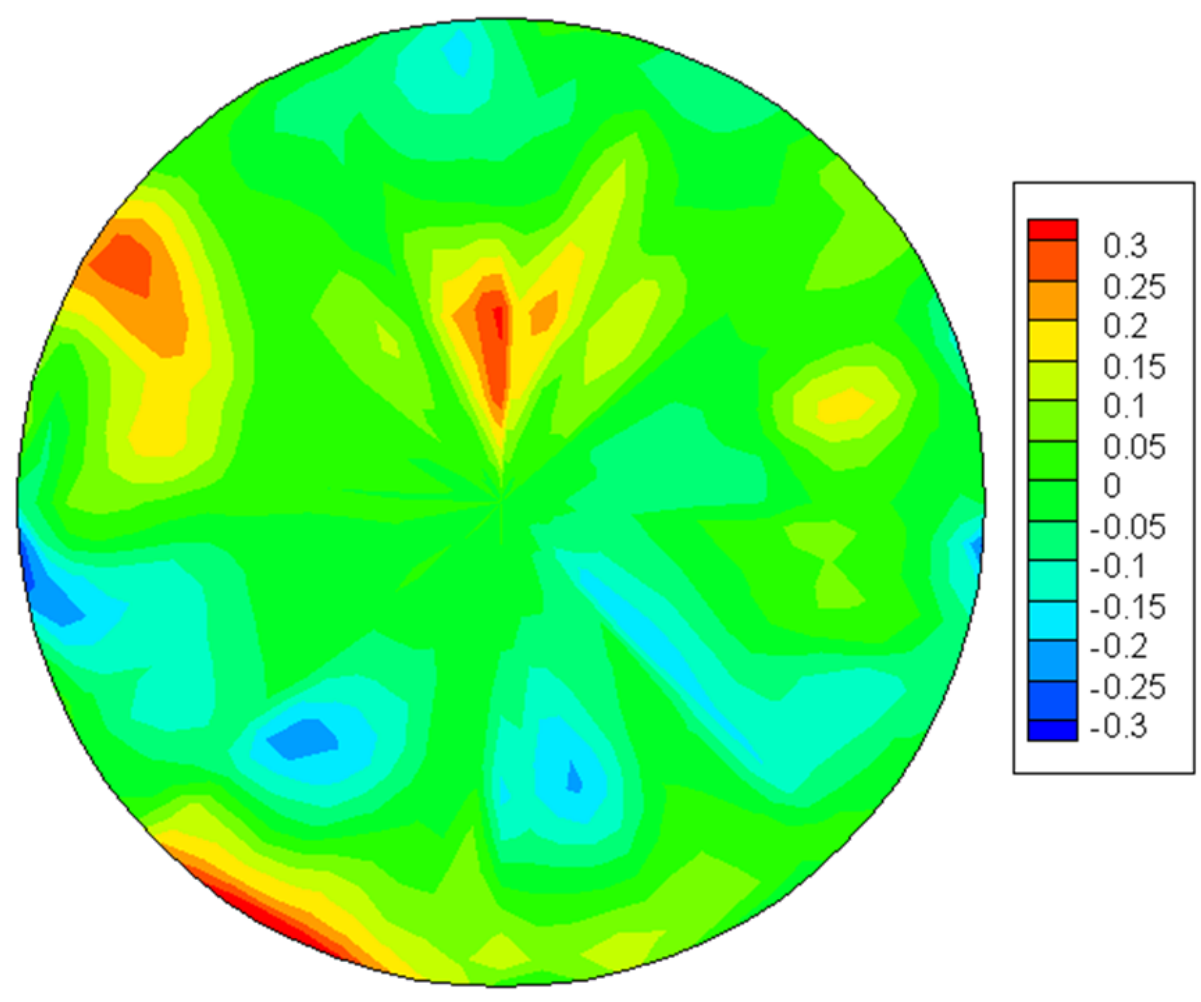

Figure D.4: Total pressure difference between dual screens and baseline (psi).

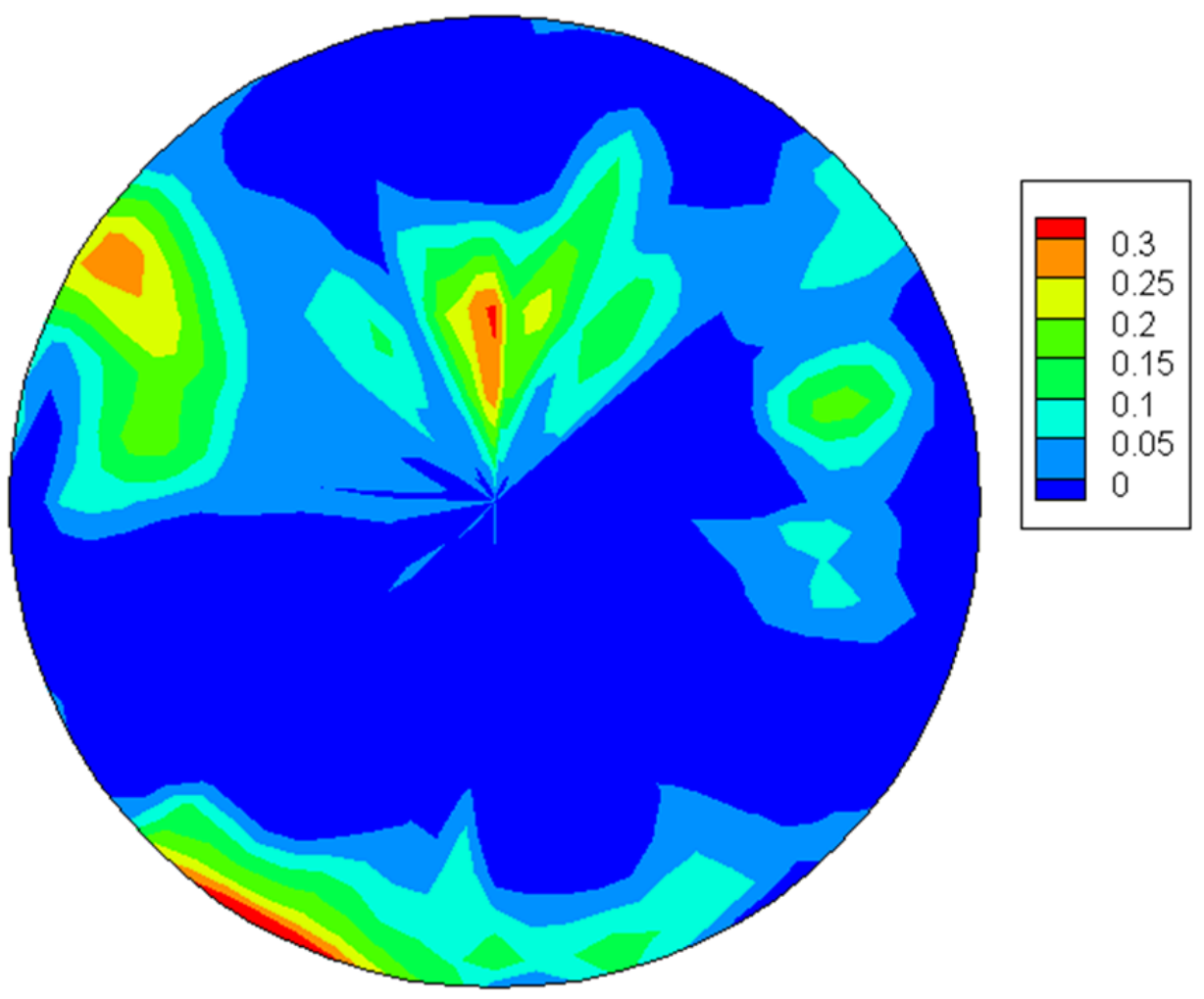

Figure D.5: Positive total pressure difference between dual screens and baseline (psi). 


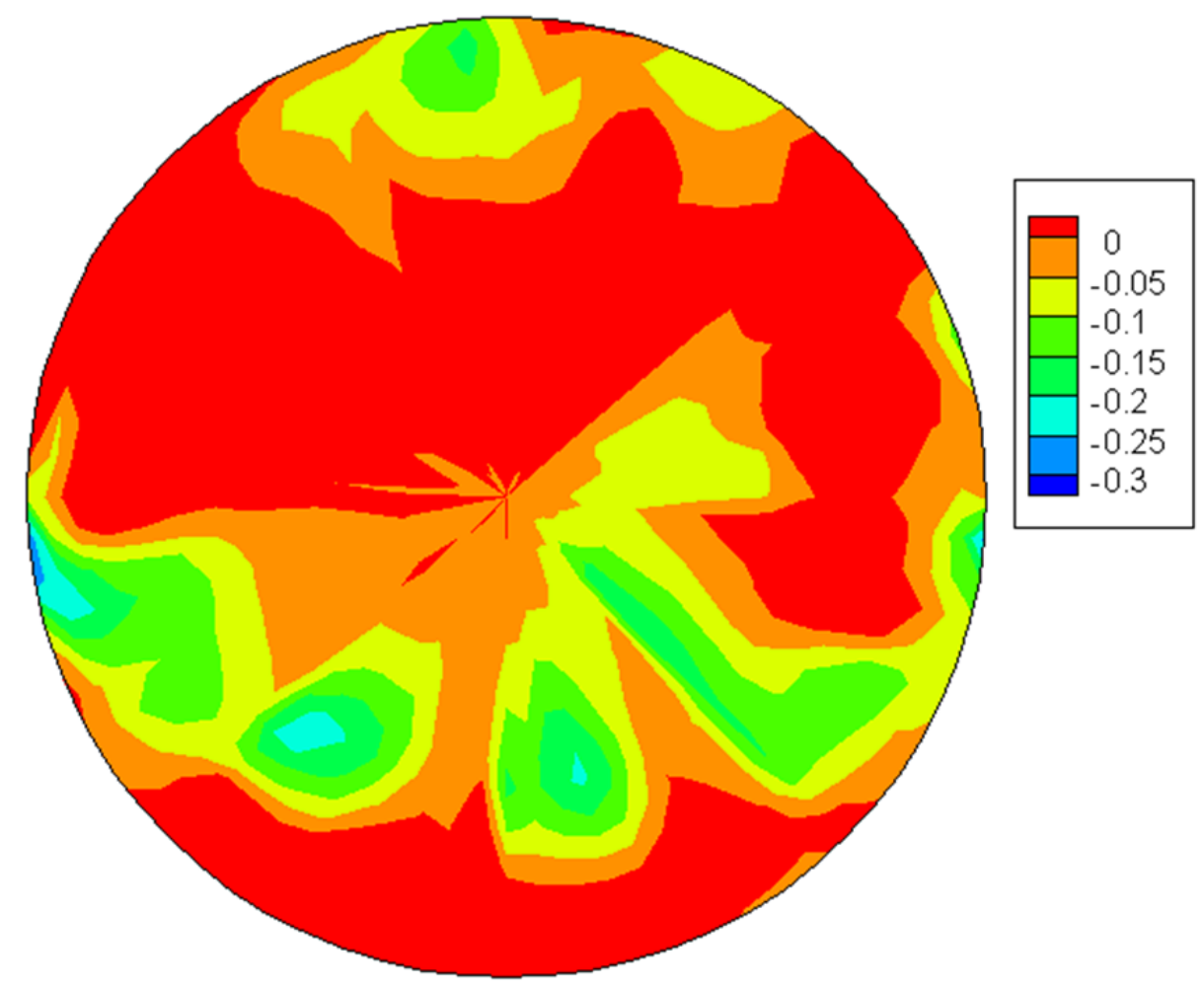

Figure D.6: Negative total pressure difference between dual screens and baseline (psi).

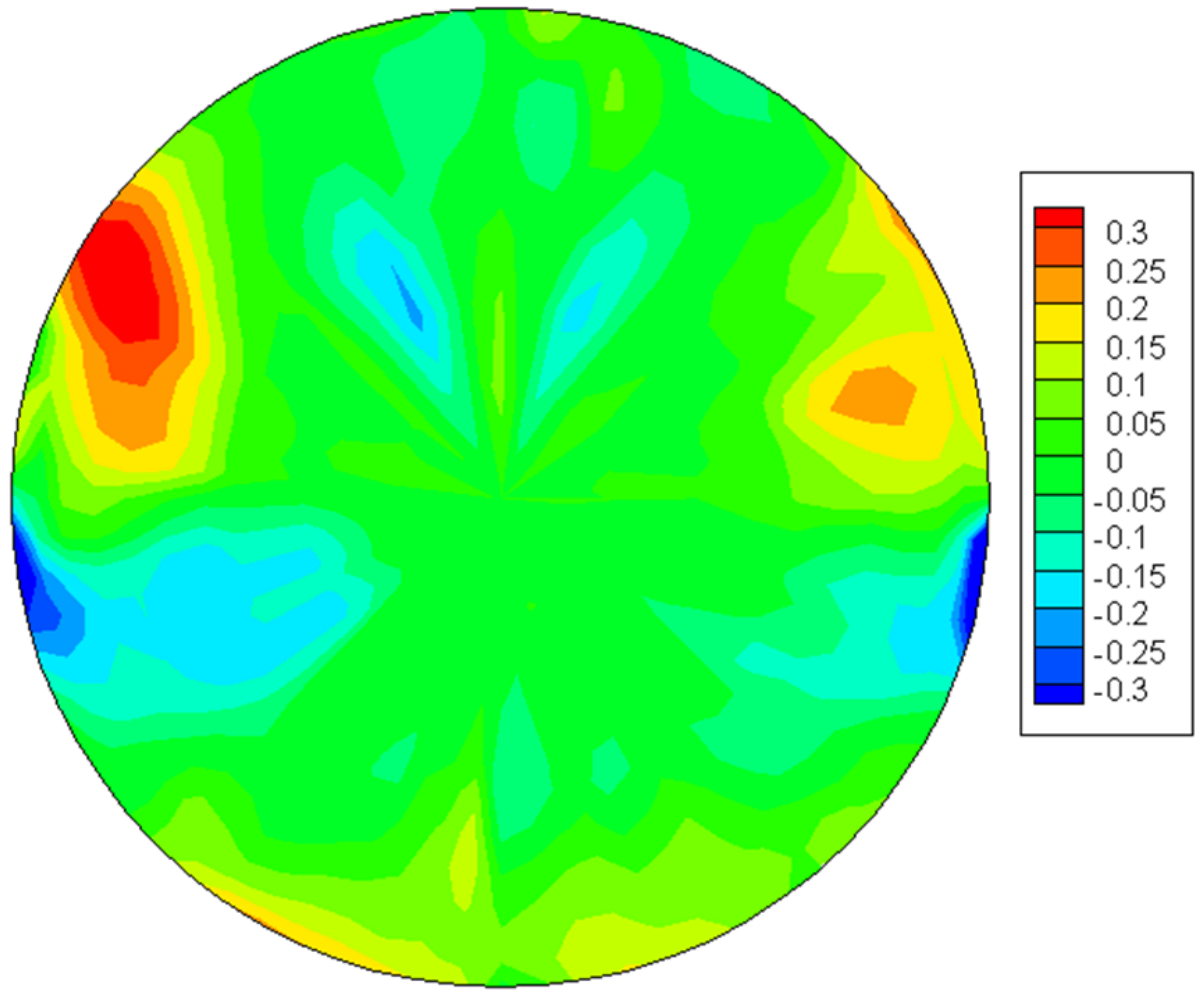

Figure D.7: Total pressure difference between high screen and baseline (psi). 


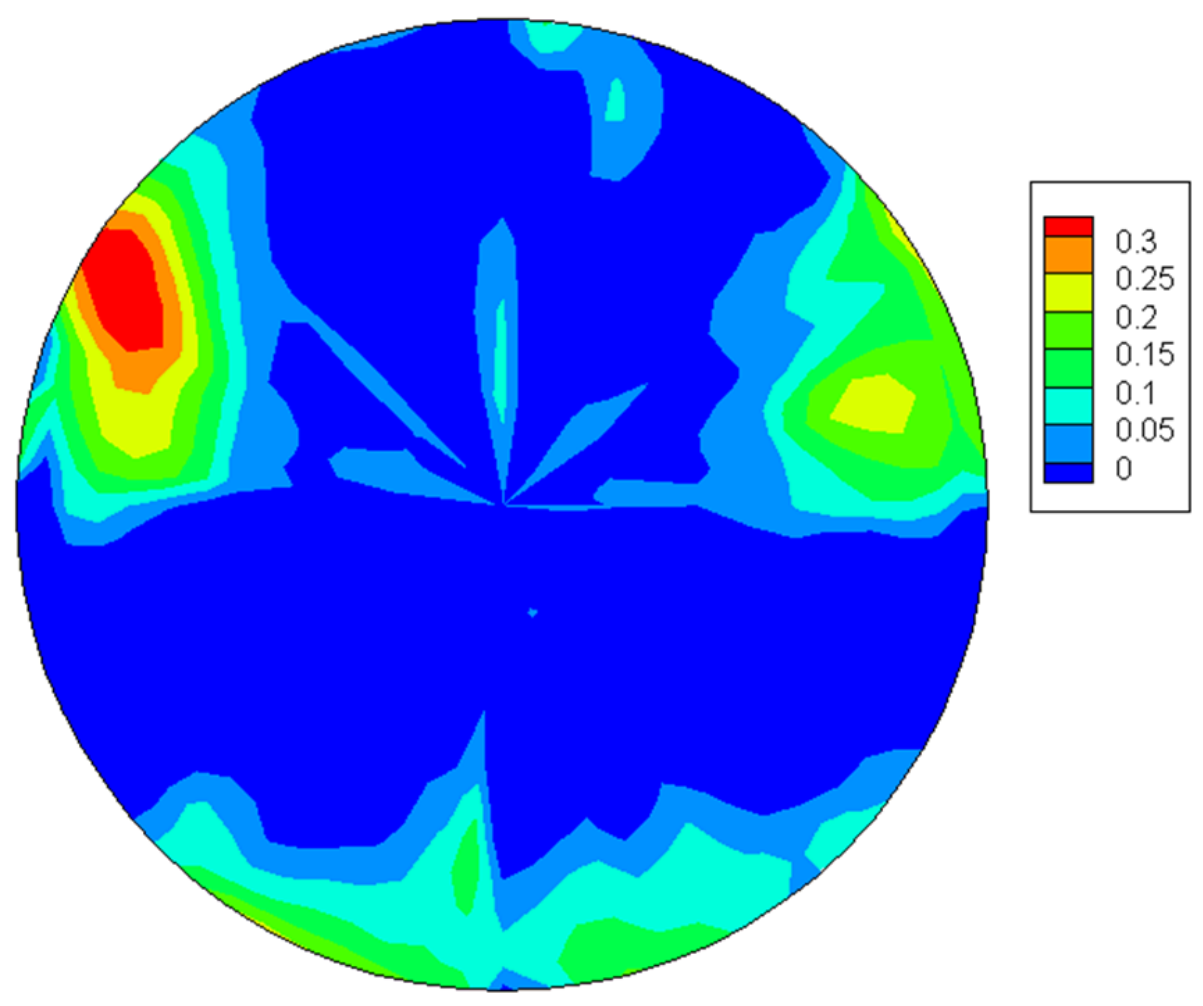

Figure D.8: Positive total pressure difference between high screen and baseline (psi).

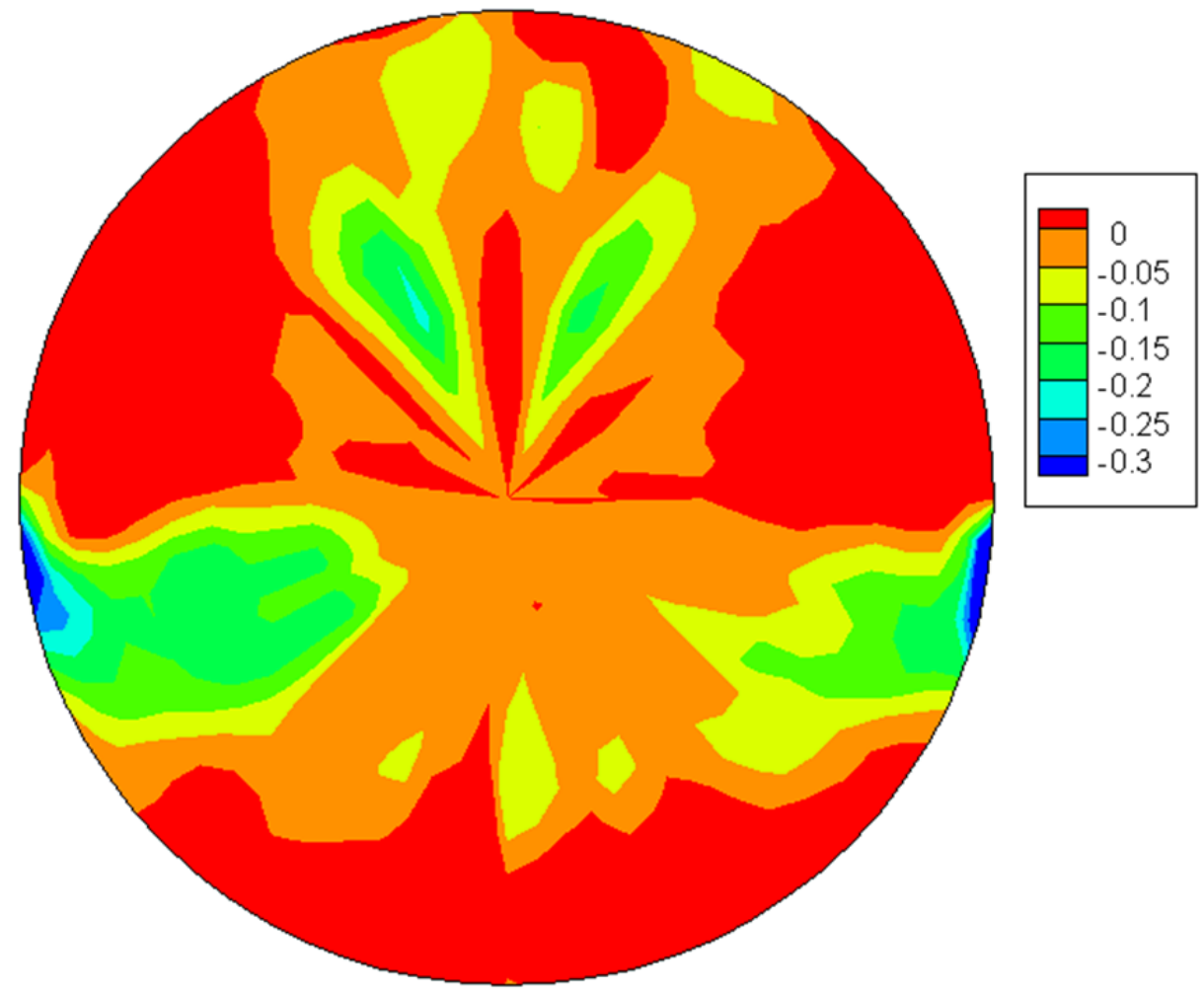

Figure D.9: Negative total pressure difference between high screen and baseline (psi). 


\section{APPENDIX E. AXIAL WALL STATIC PRESSURE PROBE LOCATIONS}

This appendix presents the locations of the axial wall static pressure taps along the upper and lower surfaces of the inside of the model diffuser. The locations are presented in Table E.1.

Table E.1: Axial wall static pressure taps locations (x/L)

\begin{tabular}{|c|c|}
\hline Upper Surface & Lower Surface \\
\hline 0.0518 & 0.0518 \\
\hline 0.0712 & 0.0709 \\
\hline 0.0903 & 0.0887 \\
\hline 0.109 & 0.105 \\
\hline 0.127 & 0.121 \\
\hline 0.145 & 0.136 \\
\hline 0.162 & 0.150 \\
\hline 0.179 & 0.165 \\
\hline 0.194 & 0.179 \\
\hline 0.210 & 0.194 \\
\hline 0.224 & 0.209 \\
\hline 0.238 & 0.225 \\
\hline 0.252 & 0.241 \\
\hline 0.266 & 0.258 \\
\hline 0.279 & 0.275 \\
\hline 0.292 & 0.293 \\
\hline 0.306 & 0.312 \\
\hline 0.320 & 0.331 \\
\hline 0.334 & 0.351 \\
\hline 0.350 & 0.371 \\
\hline 0.371 & 0.392 \\
\hline 0.383 & 0.413 \\
\hline 0.396 & 0.434 \\
\hline 0.410 & 0.451 \\
\hline 0.423 & 0.476 \\
\hline 0.437 & 0.498 \\
\hline 0.451 & 0.519 \\
\hline 0.465 & 0.540 \\
\hline 0.479 & 0.560 \\
\hline
\end{tabular}


Table E.1 (cont.)

\begin{tabular}{cc}
0.492 & 0.581 \\
0.505 & 0.601 \\
0.518 & 0.621 \\
0.530 & 0.642 \\
0.542 & 0.662 \\
0.554 & 0.683 \\
0.566 & 0.703 \\
0.578 & 0.724 \\
0.590 & 0.745 \\
0.602 & 0.766 \\
0.666 & 0.788 \\
0.929 & 0.809 \\
0.946 & 0.830 \\
0.964 & 0.851 \\
0.982 & 0.873 \\
1 & 0.890 \\
& 0.915 \\
& 0.936 \\
& 0.958 \\
& 0.979 \\
\hline
\end{tabular}

